\title{
Hypergeometric Hodge modules
}

\author{
Thomas Reichelt and Christian Sevenheck
}

\begin{abstract}
We consider mixed Hodge module structures on GKZ hypergeometric differential systems. We show that the Hodge filtration on these $\mathcal{D}$-modules is given by the order filtration, up to a suitable shift. As an application, we prove a conjecture on the existence of non-commutative Hodge structures on the reduced quantum $\mathcal{D}$-module of a nef complete intersection inside a toric variety.
\end{abstract}

\section{Introduction}

In a series of papers, Gel'fand, Graev, Kapranov and Zelevinskiǔ [GGZ87, GZK89] introduced a system of differential equations which generalize the classical differential systems satisfied by the hypergeometric functions of Gauß, Appell, Bessel and others. These generalized systems are nowadays called GKZ systems. The initial data of a GKZ system consists of a $d \times n$ integer matrix and a parameter vector $\beta$. Although the definition of a GKZ system has a combinatorial flavor, it was early realized that at least for non-resonant parameter vectors $\beta$, GKZ systems come from geometry [GKZ90]; that is, they are isomorphic to a direct image of some twisted structure sheaf on an algebraic variety. In [Rei14], the first-named author has shown that certain GKZ systems actually carry a much richer structure; namely, they underlie mixed Hodge modules in the sense of M. Saito (see [Sai90]). One of the main goals of this paper is the explicit calculation of the corresponding Hodge filtration on these modules.

An important application of GKZ systems is mirror symmetry for weak Fano complete intersections in toric varieties. We have shown in our previous papers [RS15, RS17] how to express variants of the mirror correspondence as an equivalence of differential systems of "GKZ type." However, an important point was left open in these articles: The mirror statements given there actually involve differential systems (that is, holonomic $\mathcal{D}$-modules) with some additional data, sometimes called lattices. These are constructed by a variant of the Fourier-Laplace transformation from regular holonomic filtered $\mathcal{D}$-modules. The filtration in question is the Hodge filtration on these modules, but a concrete description of it is missing in [RS15, RS17]. As a consequence, the most important Hodge-theoretic property of the differential system entering in the mirror correspondence was formulated only as a conjecture in [RS17, Conjecture 6.15]: the so-called reduced quantum $\mathcal{D}$-module, which governs certain Gromov-Witten invariants of nef complete intersections in toric varieties, conjecturally underlies a variation of non-commutative Hodge structures.

Received 29 August 2018, accepted in final form 6 May 2019.

2010 Mathematics Subject Classification 14F10 (primary), 32C38 (secondary).

Keywords: GKZ systems, Hodge modules, Hodge filtration, Radon transformation, non-commutative Hodge structures.

This journal is (C) Foundation Compositio Mathematica 2020. This article is distributed with Open Access under the terms of the Creative Commons Attribution Non-Commercial License, which permits non-commercial reuse, distribution, and reproduction in any medium, provided that the original work is properly cited. For commercial re-use, please contact the Foundation Compositio Mathematica.

The authors are supported by the DFG grants RE 3567/1-1 and SE 1114/5-1. 


\section{T. Reichelt And C. Sevenheck}

We prove this conjecture here (see Theorem 6.6); it appears as a consequence of the main result of the present paper, which determines the Hodge filtration on the GKZ systems. More precisely, as GKZ systems are defined as cyclic quotients of the Weyl algebra, we obtain (Theorem 5.35) that this Hodge filtration is given by the filtration induced from the order of differential operators up to a suitable shift. In some sense, this finishes the Hodge-theoretic study of mirror symmetry for this class of varieties since we can now express the mirror correspondence as an isomorphism of non-commutative Hodge structures, which are the correct generalization of ordinary Hodge structures in the case where the underlying differential equations acquire irregular singularities, as is the case for the quantum $\mathcal{D}$-module of weak Fano varieties (in contrast to the Calabi-Yau case).

Another application of our main result, which can be found in the two recent papers [CS19] and [CRS19], is the calculation of the so-called irregular Hodge filtration on certain 1-dimensional classical hypergeometric modules. The irregular Hodge filtration has been introduced by Sabbah [Sab18] in order to attach Hodge-type numerical invariants (namely dimensions of graded parts of a filtration) to differential systems acquiring irregular singularities. In geometric situations, like those where regular functions on quasi-projective manifolds are studied as Landau-Ginzburg models of certain quantum cohomology theories, the irregular Hodge filtration has a concrete description using certain logarithmic de Rham complexes, as has been shown by Esnault, Sabbah and $\mathrm{Yu}$ ([ESY17]; see also the discussion in [KKP17]). Classical hypergeometric systems are also the most prominent example of rigid $\mathcal{D}$-modules [Kat90], so the computation of these invariants for them is of particular interest. It turns out that confluent classical hypergeometric modules (these are precisely those with irregular singularities) are obtained from GKZ systems by a dimensional reduction and a Fourier-Laplace transformation. Using our result (that is, Theorem 5.35), one can explicitly describe the irregular Hodge filtration (and give closed formulas for irregular Hodge numbers) of certain such systems (see [CS19, Theorem 4.7] and [CRS19, Theorem 5.9] for more details).

Let us give a short overview on the content of this article and the precise statements of the main results. Notice that Section 2 provides a detailed description of these results and parts of their proofs for a rather simple example, which is related to the quantum $\mathcal{D}$-module of $\mathbb{P}^{1}$. We advise the reader to go through this example in order to understand the strategy of the proof in the general case in the main body of this article.

The main result of this paper is obtained in two major steps, which occupy Sections 4 and 5, respectively. First, we study embeddings of tori into affine spaces given by a monomial map $h_{A}: T=\left(\mathbb{C}^{*}\right)^{d} \hookrightarrow \mathbb{C}^{n},\left(t_{1}, \ldots, t_{d}\right) \mapsto\left(\underline{t}^{\underline{a}_{1}}, \ldots, \underline{t}^{\underline{a}}\right)$, where $\underline{t}^{\underline{a}} \underline{ }_{i}=\prod_{k=1}^{d} t_{j}^{a_{k i}}$ and where the matrix of columns $A=\left(\underline{a}_{i}\right)_{i=1, \ldots, n} \in \mathrm{M}(d \times n, \mathbb{Z})$ satisfies certain combinatorial properties related to the geometry of the semigroup ring $\mathbb{C}[\mathbb{N} A]$. Consider the twisted structure sheaf $\mathcal{O}_{T}^{\beta}:=$ $\mathcal{D}_{T} / \mathcal{D}_{T}\left(\partial_{t_{1}} t_{1}+\beta_{1}, \ldots, \partial_{t_{d}} t_{d}+\beta_{d}\right)$. It was shown in [SW09] that the direct image $h_{A+} \mathcal{O}_{T}^{\beta}$ has an explicit description as a Fourier-Laplace transformed GKZ system $\check{\mathcal{M}}_{A}^{\beta}$ (cf. Definition 3.5) in case the parameter vector $\beta$ is not strongly resonant (cf. Definition 3.8). We consider the corresponding direct image $\mathcal{H}^{0}\left(h_{A *}{ }^{p} \mathbb{C}_{T}^{H, \beta}\right)$ in the category of complex mixed Hodge modules and calculate its Hodge filtration (cf. Theorem 4.17) in case the parameter vector $\beta$ lies in the set of admissible parameters $\mathfrak{A}_{A}$ (cf. Formula (4.3)). More precisely, this first result can be stated as follows.

Theorem (Theorem 4.17). For $\beta \in \mathfrak{A}_{A}$, the Hodge filtration on $\check{\mathcal{M}}_{A}^{\beta}$ is equal to the order filtration shifted by $n-d$; that is,

$$
F_{p+(n-d)}^{H} \check{\mathcal{M}}_{A}^{\beta}=F_{p}^{\mathrm{ord}} \check{\mathcal{M}}_{A}^{\beta} .
$$




\section{Hypergeometric Hodge modules}

If the matrix $A$ we started with satisfies a homogeneity property, then the underlying $\mathcal{D}$ module of this mixed Hodge module is a (monodromic) Fourier-Laplace transformation of the GKZ system we are interested in. It should be noticed that Theorem 4.17 is of independent interest; its statement is related to the description of the Hodge filtration on various cohomology groups associated with singular toric varieties. We plan to discuss this question in a subsequent work. The main point in Theorem 4.17 is to determine the canonical $V$-filtration on the direct image module along the boundary divisor $\overline{\operatorname{im}\left(h_{A}\right)} \backslash \operatorname{im}\left(h_{A}\right)$, that is, the calculation of some Bernstein polynomials.

The second step, carried out in Section 5, consists in studying the behavior of a twisted structure sheaf on a torus under a certain integral transformation which generalizes the Radon transformation in [Rei14]. It is well known (see [Bry86, DE03]) that there is a close relation between the Fourier-Laplace transformation and the Radon transformation for holonomic $\mathcal{D}$ modules; however, the former does not a priori preserve the category of mixed Hodge modules, whereas the latter does. This fact is one of the main points in the proof of the existence of a mixed Hodge module structure on GKZ systems in [Rei14]. We calculate the behavior of the Hodge filtration under the various functors entering into the integral transformation functor. An essential tool for these calculations is the so-called Euler-Koszul complex (or some variants of it) as introduced in [MMW05]. We finally get the following statement for the Hodge filtration on the GKZ system $\mathcal{M}_{\widetilde{A}}^{\widetilde{\beta}}$ (cf. Theorem 5.35). We call a matrix homogeneous if all of its columns lie in an affine hyperplane. Moreover, an integer matrix is called normal if the semigroup generated by its columns is the intersection of the cone generated by these columns with the lattice generated by them (see formula (4.1) below).

ThEOREM (Theorem 5.35). Let $\widetilde{A}$ be a homogeneous, normal $(d+1) \times(n+1)$ integer matrix, $\widetilde{\beta} \in \mathfrak{A}_{\widetilde{A}}$ and $\beta_{0} \in(-1,0]$. Then the GKZ system $\mathcal{M}_{\widetilde{A}}^{\widetilde{\widetilde{A}}}$ carries the structure of a mixed Hodge module whose Hodge filtration is given by the shifted order filtration; that is,

$$
\left(\mathcal{M}_{\widetilde{A}}^{\widetilde{\beta}}, F_{\bullet}^{H}\right) \simeq\left(\mathcal{M}_{\widetilde{A}}^{\widetilde{\beta}}, F_{\bullet+d}^{\text {ord }}\right) .
$$

The second to last part of Section 5 deals with the Hodge module structure on the holonomic dual GKZ system (which, under the assumptions on the initial data, is also a GKZ system). The last subsection of Section 5 explains how one can deduce from our main result the computation of Batyrev [Bat93] of the Hodge filtration on the relative cohomology of smooth affine hypersurfaces in algebraic tori.

Finally, in Section 6, we explain the above-mentioned conjecture from [RS17] and show how its proof can be deduced from our main result (cf. Theorem 6.6). To formulate this result, consider a smooth projective toric variety $X_{\Sigma}$ and a split globally generated vector bundle $\mathcal{E}$ satisfying some positivity assumptions (ensuring, in particular, that the subvariety $Y:=s^{-1}(0)$ for a generic section $s \in \Gamma\left(X_{\Sigma}, \mathcal{E}\right)$ is smooth weak Fano). Then we have the reduced quantum $\mathcal{D}$-module $\overline{\mathrm{QDM}}\left(X_{\Sigma}, \mathcal{E}\right)$ (see $[\mathrm{RS} 17$, Section 4.1]) and the mirror map Mir (see Theorem 6.5 below as well as [RS17, Theorem 6.9] and the references therein). We get the following result.

TheOREM (Theorem 6.6). Let $X_{\Sigma}$ be a $k$-dimensional smooth, toric variety, and let $\mathcal{L}_{1}, \ldots, \mathcal{L}_{l}$ be globally generated line bundles such that $-K_{X_{\Sigma}}-\mathcal{E}$ is nef, where $\mathcal{E}=\oplus_{j=1}^{l} \mathcal{L}_{j}$, with $\mathcal{L}_{j}$ ample for

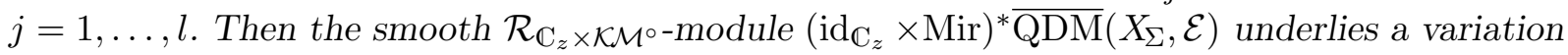
of pure polarized non-commutative Hodge structures.

While we were working on this paper, a preprint of T. Mochizuki [Moc15b] appeared where [RS17, Conjecture 6.15] is shown with rather different methods. The arguments in his paper work 


\section{T. Reichelt And C. Sevenheck}

entirely in the category of mixed twistor modules, using the full strength of [Moc15a]. It seems possible from his approach to obtain our main result by considering equivariant twistor modules [Moc15b, Section 6.4]. However, the treatment of the regular case in loc. cit. (that is, the case of mixed Hodge modules) seems to be done in a setup that is more restrictive in two ways: First, there is an extra assumption [Moc15b, Assumption 6.51] excluding the calculation of the Hodge filtration on GKZ systems for examples coming from local mirror symmetry (however, the mirror symmetry consequence, that is, the proof of [RS17, Conjecture 6.15], is covered by [Moc15b]). On the other hand, there is no discussion on GKZ systems (or its twistor versions) for non-zero parameter vectors $\beta$ in [Moc15b], in contrast to our main result (that is, in contrast to Theorem 5.35, which holds for non-zero parameters satisfying a natural combinatorial condition). Besides, we feel that the calculation of the Hodge filtration on GKZ systems which only uses properties of the category of mixed Hodge modules (not passing via twistor modules) may be of independent interest.

To finish this introduction, we introduce some notation and conventions used throughout the paper. Let $X$ be a smooth algebraic variety over $\mathbb{C}$ of dimension $d_{X}$. We denote by $M\left(\mathcal{D}_{X}\right)$ the abelian category of algebraic left $\mathcal{D}_{X}$-modules on $X$ and denote the abelian subcategory of (regular) holonomic $\mathcal{D}_{X}$-modules by $M_{h}\left(\mathcal{D}_{X}\right)$ (respectively, $\left(M_{r h}\left(\mathcal{D}_{X}\right)\right)$. The full triangulated subcategory in $D^{b}\left(\mathcal{D}_{X}\right)$ consisting of objects with (regular) holonomic cohomology is denoted by $D_{h}^{b}\left(\mathcal{D}_{X}\right)$ (respectively, $D_{r h}^{b}\left(\mathcal{D}_{X}\right)$ ).

Let $f: X \rightarrow Y$ be a map between smooth algebraic varieties. Let $M \in D^{b}\left(\mathcal{D}_{X}\right)$ and $N \in$ $D^{b}\left(\mathcal{D}_{Y}\right)$; then we denote by

$$
f_{+} M:=R f_{*}\left(\mathcal{D}_{Y \leftarrow X} \stackrel{L}{\otimes} M\right), \quad \text { respectively } \quad f^{+} M:=\mathcal{D}_{X \rightarrow Y} \stackrel{L}{\otimes} f^{-1} M\left[d_{X}-d_{Y}\right]
$$

the direct, respectively inverse image, for $\mathcal{D}$-modules. Recall that the functors $f_{+}$and $f^{+}$preserve (regular) holonomicity (see, for example, [HTT08, Theorem 3.2.3]).

We denote by $\mathbb{D}: D_{h}^{b}\left(\mathcal{D}_{X}\right) \rightarrow\left(D_{h}^{b}\left(\mathcal{D}_{X}\right)\right)^{\text {opp }}$ the holonomic duality functor. Recall that for a single holonomic $\mathcal{D}_{X}$-module $M$, the holonomic dual is also a single holonomic $\mathcal{D}_{X}$-module [HTT08, Proposition 3.2.1] and that holonomic duality preserves regular holonomicity [HTT08, Theorem 6.1.10].

For a morphism $f: X \rightarrow Y$ between smooth algebraic varieties, we additionally define the functors $f_{\dagger}:=\mathbb{D} \circ f_{+} \circ \mathbb{D}$ and $f^{\dagger}:=\mathbb{D} \circ f^{+} \circ \mathbb{D}$.

Let $M F\left(\mathcal{D}_{X}\right)$ be the category of filtered $\mathcal{D}_{X}$-modules $(M, F)$ where the ascending filtration $F$. satisfies

(1) $F_{p} M=0$ for $p \ll 0$,

(2) $\bigcup_{p} F_{p} M=M$,

(3) $\left(F_{p} \mathcal{D}_{X}\right) F_{q} M \subset F_{p+q} M$ for $p \in \mathbb{Z}_{\geqslant 0}$ and $q \in \mathbb{Z}$,

where $F_{\bullet} \mathcal{D}_{X}$ is the filtration by the order of the differential operator.

We denote by $\operatorname{MHM}(X)$ the abelian category of algebraic mixed Hodge modules and by $D^{b} \operatorname{MHM}(X)$ the corresponding bounded derived category. The forgetful functor to the bounded derived category of regular holonomic $\mathcal{D}$-modules is denoted by

$$
\operatorname{Dmod}: D^{b} \operatorname{MHM}(X) \longrightarrow D_{r h}^{b}\left(\mathcal{D}_{X}\right) \text {. }
$$

For each morphism $f: X \rightarrow Y$ between complex algebraic varieties, there are induced functors

$$
f_{*}, f_{!}: D^{b} \operatorname{MHM}(X) \longrightarrow D^{b} \operatorname{MHM}(Y)
$$




\section{Hypergeometric Hodge modules}

and

$$
f^{*}, f^{!}: D^{b} \operatorname{MHM}(Y) \rightarrow D^{b} \operatorname{MHM}(X),
$$

which are interchanged by $\mathbb{D}$. The functors $f_{*}, f_{!}, f^{*}, f^{!}$lift the analogous functors $f_{+}, f_{\dagger}, f^{\dagger}, f^{+}$ on $D_{\mathrm{rh}}^{b}\left(\mathcal{D}_{X}\right)$. Let $\mathbb{Q}_{\mathrm{pt}}^{H}$ be the unique mixed Hodge structure with $\operatorname{Gr}_{i}^{W}=\operatorname{Gr}_{i}^{F}=0$ for $i \neq 0$ and underlying vector space $\mathbb{Q}$. Denote by $a_{X}: X \longrightarrow\{\mathrm{pt}\}$ the map to the point, and set

$$
\mathbb{Q}_{X}^{H}:=a_{X}^{*} \mathbb{Q}_{\mathrm{pt}}^{H} \text {. }
$$

The shifted object ${ }^{p} \mathbb{Q}_{X}^{H}:=\mathbb{Q}_{X}^{H}\left[d_{X}\right]$ lies in $\operatorname{MHM}(X)$ and is equal to $\left(\mathcal{O}_{X}, F, \mathbb{Q}_{X}\left[d_{X}\right], W\right)$ with $\operatorname{Gr}_{p}^{F}=0$ for $p \neq 0$ and $\operatorname{Gr}_{i}^{W}=0$ for $i \neq d_{X}$. We have $\mathbb{D} \mathbb{Q}_{X}^{H} \simeq a_{X}^{!} \mathbb{Q}_{\mathrm{pt}}^{H}$ and, since $X$ is smooth, the isomorphism

$$
\mathbb{D} \mathbb{Q}_{X}^{H} \simeq \mathbb{Q}_{X}^{H}\left(d_{X}\right)\left[2 d_{X}\right] .
$$

Here $\left(d_{X}\right)$ denotes the Tate twist (see, for example, [Sai90, p. 257]).

We also have to consider the category $\operatorname{MHM}(X, \mathbb{C})$ of complex mixed Hodge modules which can be defined as follows [DS13, Definition 3.2.1]: First, note that one can naturally extend the notion of a $\mathbb{Q}$-mixed Hodge module (that is, an object of $\operatorname{MHM}(X)$ ) to a $\mathbb{R}$-mixed Hodge module, due to the work of Mochizuki on mixed twistor modules (see, in particular, [Moc15a, Section 13.5], where the notion of a $K$-mixed Hodge module is considered for $K$ any subfield of $\mathbb{R}$ ). Then we say that a filtered $\mathcal{D}_{X}$-module $\left(M, F_{\bullet}\right)$ underlies a complex mixed Hodge module if it is a direct summand of a filtered $\mathcal{D}_{X}$-module that underlies an $\mathbb{R}$-mixed Hodge module. Many properties of the category of $\mathbb{R}$-mixed Hodge modules carry over to $\operatorname{MHM}(X, \mathbb{C})$ since they are stable by direct summands.

Let $T=\left(\mathbb{C}^{*}\right)^{d}$ be a torus with coordinates $t_{1}, \ldots, t_{d}$, and let $\beta \in \mathbb{R}^{d}$. We denote by $\mathcal{O}_{T}^{\beta}$ the $\mathcal{D}_{T}$-module

$$
\mathcal{O}_{T}^{\beta}=\mathcal{D}_{T} /\left(\left(\partial_{t_{i}} t_{i}+\beta_{i}\right)_{i=1, \ldots, d}\right)
$$

and by $\mathbb{C}_{T}^{H, \beta}$ the complex Hodge module $\left(\mathcal{O}_{T}^{\beta}, F, W\right)$ with $\operatorname{Gr}_{p}^{F} \mathbb{C}_{T}^{H, \beta}=0$ for $p \neq 0$ and $\operatorname{Gr}_{i}^{W} \mathbb{C}_{T}^{H, \beta}$ $=0$ for $i \neq d$. Finally, we set ${ }^{p} \mathbb{C}_{T}^{H, \beta}:=\mathbb{C}_{T}^{H, \beta}\left[d_{T}\right]$.

\section{A guiding example}

In this section, we intend to discuss a particular example, related to the quantum differential equation of $\mathbb{P}^{1}$, where most of the techniques used in the main body of the paper can be written down quite explicitly. We hope that this section will help the reader to find his way through the technical difficulties of the paper.

Let $\widetilde{A}$ be the following $2 \times 3$ matrix with integer entries:

$$
\left(\begin{array}{ccc}
1 & 1 & 1 \\
0 & 1 & -1
\end{array}\right)
$$

As explained in Definition 3.1 below, any $d \times n$ integer matrix $A$ together with a vector $\beta \in \mathbb{C}^{d}$

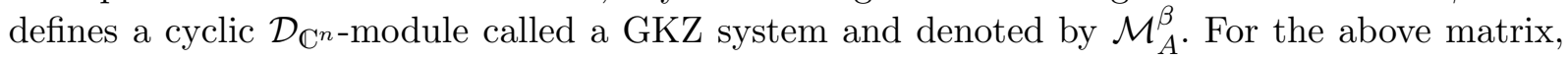
this system for the vector $\beta=0$ is given by

$$
\mathcal{M}_{\widetilde{A}}^{0}=\mathcal{D}_{\mathbb{C}^{3}} /\left(\partial_{\lambda_{0}}^{2}-\partial_{\lambda_{1}} \partial_{\lambda_{2}}, \lambda_{0} \partial_{\lambda_{0}}+\lambda_{1} \partial_{\lambda_{1}}+\lambda_{2} \partial_{\lambda_{2}}, \lambda_{1} \partial_{\lambda_{1}}-\lambda_{2} \partial_{\lambda_{2}}\right) .
$$

It is well known that $\mathcal{M}_{\widetilde{A}}^{0}$ is holonomic [Ado94] and regular [SW08]. Moreover, it follows from [Rei14, Theorem 3.5] that $\mathcal{M}_{\widetilde{A}}^{0}$ underlies a mixed Hodge module ${ }^{H} \mathcal{M}_{\widetilde{A}}^{0} \in \operatorname{MHM}\left(\mathbb{C}^{3}\right)$. The purpose 


\section{T. Reichelt And C. Sevenheck}

of this introductory section is to explain and partly prove the following statement, which is a very special case of the main theorem of this paper (Theorem 5.35).

Theorem 2.1. We have an isomorphism of filtered $\mathcal{D}_{\mathbb{C}^{3} \text {-modules }}$

$$
\left(\mathcal{M}_{\widetilde{A}}^{0}, F_{\bullet}^{H}\right) \simeq\left(\mathcal{M}_{\widetilde{A}}^{0}, F_{\bullet+1}^{\text {ord }}\right),
$$

where $F_{\bullet}^{H}$ denotes the filtration such that the filtered modules $\left(\mathcal{M}_{\widetilde{A}}^{0}, F_{\bullet}^{H}\right)$ underly the mixed Hodge module ${ }^{H} \mathcal{M}_{\widetilde{A}}^{0}$ and where $F_{\bullet}^{\text {ord }}$ is the filtration induced on $\mathcal{M}_{\widetilde{A}}^{0}$ by the filtration on $\mathcal{D}_{\mathbb{C}^{3}}$ by orders of differential operators.

The proof of this theorem will be done in several steps that parallel the main steps of the proof of Theorem 5.35. The two major simplifications are that we are dealing with the very special matrix $\widetilde{A}$, whereas in Theorem 5.35 any $(d+1) \times(n+1)$ matrix $\widetilde{A}$ satisfying some combinatorial conditions is considered, and moreover we restrict to the parameter value $\beta=0$ (compare with the general definition of GKZ systems $\mathcal{M}_{\widetilde{A}}^{\beta}$ in Definition 3.1 and with Definition 3.5 for the sheaf $\check{\mathcal{M}}_{\widetilde{A}}^{\beta}$ ). This avoids considering some rather involved combinatorial condition relating $\widetilde{A}$ and $\beta$ (see the definition of the set $\mathfrak{A}_{\widetilde{A}}$ in equation (4.3) below).

We first consider the morphism

$$
h_{\widetilde{A}}:\left(\mathbb{C}^{*}\right)^{2} \longrightarrow \mathbb{C}^{3}=: W, \quad\left(t_{0}, t_{1}\right) \longmapsto\left(t_{0}, t_{0} \cdot t_{1}, t_{0} \cdot t_{1}^{-1}\right)=:\left(w_{0}, w_{1}, w_{2}\right),
$$

where the exponents of the monomials in the components of the map are exactly the columns of $\widetilde{A}$.

As explained in more detail in Theorem 3.9 and Proposition 3.11, it follows from a result of Schulze and Walther [SW09] that we have the following isomorphism of $\mathcal{D}_{W}$-modules:

$$
\begin{aligned}
h_{\widetilde{A},+} \mathcal{O}_{\left(\mathbb{C}^{*}\right)^{2}} & \cong h_{\widetilde{A},+} \mathcal{D}_{\left(\mathbb{C}^{*}\right)^{2}} /\left(\partial_{t_{0}} t_{0}+2, \partial_{t_{1}} t_{1}-1\right) \\
& \stackrel{!}{\cong} \mathcal{D}_{W} /\left(w_{0}^{2}-w_{1} w_{2}, \partial_{w_{0}} w_{0}+\partial_{w_{1}} w_{1}+\partial_{w_{2}} w_{2}, \partial_{w_{1}} w_{1}-\partial_{w_{2}} w_{2}\right)=: \check{\mathcal{M}}_{\widetilde{A}}^{0} .
\end{aligned}
$$

The main point in this result is to show that (left) multiplication by $w_{0}$ is invertible on $\check{\mathcal{M}}_{\widetilde{A}}^{0}$. The isomorphism then follows since the map $h_{\widetilde{A}}$ can be decomposed as

$$
h_{\widetilde{A}}:\left(\mathbb{C}^{*}\right)^{2} \stackrel{h_{\widetilde{A}, 1}}{\longrightarrow} \mathbb{C}^{*} \times \mathbb{C}^{2} \stackrel{h_{\widetilde{A}, 2}}{\longrightarrow} W,
$$

where both $h_{\widetilde{A}, 1}$ and $h_{\widetilde{A}, 2}$ are embeddings and where $h_{\widetilde{A}, 1}$ sends $\left(t_{0}, t_{1}\right)$ to $\left(t_{0}, t_{0} \cdot t_{1}, t_{0} \cdot t_{1}^{-1}\right)$ and is closed (this follows since the map $\mathbb{C}^{*} \rightarrow \mathbb{C}^{2}, t \mapsto\left(t, t^{-1}\right)$ is a closed embedding), whereas $h_{\widetilde{A}, 2}$ is the canonical open embedding of $\mathbb{C}^{*} \times \mathbb{C}^{2}$ into $W$.

A second consequence of Proposition 3.11 is that $\check{\mathcal{M}}_{\widetilde{A}}^{0}$ underlies a mixed Hodge module on $W$, namely the object $h_{\widetilde{A}, *}{ }^{p} \mathbb{Q}_{T}^{H} \in \operatorname{MHM}(W)$. A first step to prove Theorem 2.1 is to compute the Hodge filtration on $\check{\mathcal{M}}_{\widetilde{A}}^{0}$. This task can be divided into two steps: First, we have to compute the Hodge filtration on the module ${ }^{*} \check{\mathcal{M}}_{\widetilde{A}}^{0}:=h_{\widetilde{A}, 1,+} \mathcal{O}_{\left(\mathbb{C}^{*}\right)^{2}}$ which underlies the mixed Hodge module $h_{\widetilde{A}, 1, *}^{p} \mathbb{Q}_{T}^{H} \in \operatorname{MHM}\left(\mathbb{C}^{*} \times \mathbb{C}^{2}\right)$. This is done via a rather direct argument since $h_{\widetilde{A}, 1}$ is closed. The second step, which is more delicate, is to obtain from this the Hodge filtration on $h_{\widetilde{A}, 2,+}{ }^{*} \check{\mathcal{M}}_{\widetilde{A}}^{0}=\check{\mathcal{M}}_{\widetilde{A}}^{0}$.

Here we are faced with the fundamental problem of extending a mixed Hodge module from the complement of a (smooth) divisor to the total space. While this operation is easily understood at the level of $\mathcal{D}$-modules, one cannot simply use the direct image functors for $\mathcal{O}$-modules to 


\section{Hypergeometric Hodge MOdules}

calculate the extension of the filtration steps of the Hodge filtration since those are by definition $\mathcal{O}$-coherent, a property that is lost under direct images of open embeddings. As explained in more detail at the beginning of Subsection 4.3, this problem is solved by intersecting the direct image with the canonical $V$-filtration along the divisor in question. In order to compute the Hodge filtration on $h_{\widetilde{A}, 2,+}{ }^{*} \check{\mathcal{M}}_{\widetilde{A}}^{0}$, we thus have to calculate this $V$-filtration along $w_{1}=0$.

Notice that in the main body of the text, we follow a slightly different strategy, due to the fact that the factorization used above of $h_{\widetilde{A}}$ into a closed and open embedding may look different depending on the shape of the matrix $\widetilde{A}$. In general, one can always consider the factorization into a map between tori, which is a closed embedding (which would be the map from $\left(\mathbb{C}^{*}\right)^{2}$ to $\left(\mathbb{C}^{*}\right)^{3}$ in the above example) followed by the canonical open embedding from the torus into affine space. The latter, however, is the extension over a normal crossing divisor, which is not smooth. In order to apply the techniques sketched above, one has to compose further with a graph embedding with respect to the equation of the normal crossing divisor (see diagram (4.10) and the arguments following it).

The first (easy) step of the calculation of the Hodge filtration on $\check{\mathcal{M}}_{\widetilde{A}}^{0}$ can be formulated as follows. (Compare with Lemma 4.14 for the general case.)

Lemma 2.2. The direct image $\mathcal{H}^{0} h_{\widetilde{A}, 1,+} \mathcal{O}_{\left(\mathbb{C}^{*}\right)^{2}}$ is isomorphic to the cyclic $\mathcal{D}_{\mathbb{C}^{*} \times \mathbb{C}^{2} \text {-module }}$

$$
\mathcal{D}_{\mathbb{C}^{*} \times \mathbb{C}^{2}} /\left(w_{0}^{2}-w_{1} w_{2}, \partial_{w_{0}} w_{0}+\partial_{w_{1}} w_{1}+\partial_{w_{2}} w_{2}, \partial_{w_{1}} w_{1}-\partial_{w_{2}} w_{2}\right)
$$

and, under this isomorphism, the Hodge filtration on this module is given by the induced order filtration, shifted by one; that is, we have

$$
F_{p}^{H} \mathcal{H}^{0} h_{\widetilde{A}, 1,+} \mathcal{O}_{\left(\mathbb{C}^{*}\right)^{2}}=F_{p-1}^{\text {ord }}\left[\mathcal{D}_{\mathbb{C}^{*} \times \mathbb{C}^{2}} /\left(w_{0}^{2}-w_{1} w_{2}, \partial_{w_{0}} w_{0}+\partial_{w_{1}} w_{1}+\partial_{w_{2}} w_{2}, \partial_{w_{1}} w_{1}-\partial_{w_{2}} w_{2}\right)\right] .
$$

Proof. We can factor $h_{\widetilde{A}, 1}$ further as $h_{\widetilde{A}, 1}=\widetilde{\widetilde{h}}_{\widetilde{A}, 1} \circ \widetilde{h}_{\widetilde{A}, 1}$, where

$$
\widetilde{h}_{\widetilde{A}, 1}:\left(\mathbb{C}^{*}\right)^{2} \longrightarrow\left(\mathbb{C}^{*}\right)^{3}, \quad\left(t_{0}, t_{1}\right) \longmapsto\left(t_{0}, t_{0} \cdot t_{1}, t_{0} \cdot t_{1}^{-1}\right),
$$

and where $\widetilde{\widetilde{h}}_{\widetilde{A}, 1}:\left(\mathbb{C}^{*}\right)^{3} \hookrightarrow \mathbb{C}^{*} \times \mathbb{C}^{2}$ is the canonical open embedding. Then, since $h_{\widetilde{A}, 1}$ is a closed embedding, we know that the support of ${ }^{*} \mathcal{M}_{\widetilde{A}}^{0}$ is disjoint from the divisor $\left(\mathbb{C}^{*} \times \mathbb{C}^{2}\right) \backslash\left(\mathbb{C}^{*}\right)^{3}$, which implies that $F_{p}^{H *} \mathcal{M}_{\widetilde{A}}^{0}=\widetilde{\widetilde{h}}_{\widetilde{A}, 1, *} F_{p}^{H} \widetilde{h}_{\widetilde{A}, 1,+} \mathcal{O}_{\left(\mathbb{C}^{*}\right)^{2}}$. It therefore suffices to determine the filtration steps $F_{p}^{H} \widetilde{h}_{\widetilde{A}, 1,+} \mathcal{O}_{\left(\mathbb{C}^{*}\right)^{2}}$ or, more precisely, to show that

$$
F_{p}^{H} \widetilde{h}_{\widetilde{A}, 1,+} \mathcal{O}_{\left(\mathbb{C}^{*}\right)^{2}}=F_{p-1}^{\text {ord }}\left[\mathcal{D}_{\left(\mathbb{C}^{*}\right)^{3}} /\left(w_{0}^{2}-w_{1} w_{2}, \partial_{w_{0}} w_{0}+\partial_{w_{1}} w_{1}+\partial_{w_{2}} w_{2}, \partial_{w_{1}} w_{1}-\partial_{w_{2}} w_{2}\right)\right] .
$$

Consider the coordinate change

$$
\phi:\left(\mathbb{C}^{*}\right)^{3} \longrightarrow\left(\mathbb{C}^{*}\right)^{3}, \quad\left(w_{0}, w_{1}, w_{2}\right) \longmapsto\left(w_{0}, w_{1} / w_{0}, w_{1} w_{2} / w_{0}^{2}\right)=:\left(u_{0}, u_{1}, u_{2}\right) ;
$$

then $\left(\phi \circ \widetilde{h}_{\widetilde{A}, 1}\right)\left(t_{0}, t_{1}\right)=\left(t_{0}, t_{1}, 1\right)$ and

$$
\left(\phi \circ \widetilde{h}_{\widetilde{A}, 1}\right)_{+} \mathcal{O}_{\left(\mathbb{C}^{*}\right)^{2}}=\mathcal{D}_{\left(\mathbb{C}^{*}\right)^{3}} /\left(u_{2}-1, \partial_{u_{0}} u_{0}+2, \partial_{u_{1}} u_{1}\right)=\left(\mathcal{D}_{\left(\mathbb{C}^{*}\right)^{2}} /\left(\partial_{u_{0}} u_{0}+2, \partial_{u_{1}} u_{1}\right)\right)\left[\partial_{u_{2}}\right] .
$$

According to [Sai93, formula (1.8.6)], we have

$$
F_{p+1}^{H}\left(\phi \circ \widetilde{h}_{\widetilde{A}, 1}\right)_{+} \mathcal{O}_{\left(\mathbb{C}^{*}\right)^{2}}=\sum_{p_{1}+p_{2}=p} F_{p_{1}}^{H}\left(\mathcal{D}_{\left(\mathbb{C}^{*}\right)^{2}} /\left(\partial_{u_{0}} u_{0}+2, \partial_{u_{1}} u_{1}\right) \partial_{u_{2}}^{p_{2}} .\right.
$$




\section{T. Reichelt And C. Sevenheck}

Since $F_{p_{1}}^{H}\left(\mathcal{D}_{\left(\mathbb{C}^{*}\right)^{3}} /\left(\partial_{u_{0}} u_{0}, \partial_{u_{1}} u_{1}\right)\right)=F_{p_{1}}^{\text {ord }}\left(\mathcal{D}_{\left(\mathbb{C}^{*}\right)^{3}} /\left(\partial_{u_{0}} u_{0}, \partial_{u_{1}} u_{1}\right)\right.$, we obtain from the above formula that

$$
F_{p}^{H}\left(\phi \circ \widetilde{h}_{\widetilde{A}, 1}\right)_{+} \mathcal{O}_{\left(\mathbb{C}^{*}\right)^{2}}=F_{p-1}^{\text {ord }}\left(\mathcal{D}_{\left(\mathbb{C}^{*}\right)^{3}} /\left(u_{2}-1, \partial_{u_{0}} u_{0}, \partial_{u_{1}} u_{1}\right)\right) .
$$

Since $\phi$ is invertible, we obtain that the Hodge filtration on $\phi_{+}^{-1}\left(\left(\phi \circ \widetilde{h}_{\widetilde{A}, 1}\right)_{+} \mathcal{O}_{\left(\mathbb{C}^{*}\right)^{2}}\right)=\widetilde{h}_{\widetilde{A}, 1,+} \mathcal{O}_{\left(\mathbb{C}^{*}\right)^{2}}$ is the order filtration on $\mathcal{D}_{\left(\mathbb{C}^{*}\right)^{3}} /\left(w_{0}^{2}-w_{1} w_{2}, \partial_{w_{0}} w_{0}+\partial_{w_{1}} w_{1}+\partial_{w_{2}} w_{2}, \partial_{w_{1}} w_{1}-\partial_{w_{2}} w_{2}\right)$, shifted by one. As discussed above, the closure of the support of $\widetilde{h}_{\widetilde{A}, 1,+} \mathcal{O}_{\left(\mathbb{C}^{*}\right)^{2}}$ in $\mathbb{C}^{*} \times \mathbb{C}^{2}$ lies entirely in the torus $\left(\mathbb{C}^{*}\right)^{3}$; therefore, we obtain

$$
F_{p}^{H} h_{\widetilde{A}, 1,+} \mathcal{O}_{\left(\mathbb{C}^{*}\right)^{2}}=F_{p-1}^{\text {ord }}\left[\mathcal{D}_{\mathbb{C}^{*} \times \mathbb{C}^{2}} /\left(w_{0}^{2}-w_{1} w_{2}, \partial_{w_{0}} w_{0}+\partial_{w_{1}} w_{1}+\partial_{w_{2}} w_{2}, \partial_{w_{1}} w_{1}-\partial_{w_{2}} w_{2}\right)\right],
$$

as required.

The next step is to compute the Hodge filtration of the open direct image $h_{\widetilde{A}, 2,+}{ }^{*} \check{\mathcal{M}}_{\widetilde{A}}^{0}=\check{\mathcal{M}}_{\widetilde{A}}^{0}$. As mentioned above, this requires information on the canonical $V$-filtration of the module $\check{\mathcal{M}}_{\widetilde{A}}^{0}$ with respect to the smooth divisor $\left\{w_{0}=0\right\}$. More precisely, we have the following important formula (see formula (4.9), as well as [Sai93, Proposition 4.2.]):

$$
F_{p}^{H} \check{\mathcal{M}}_{\widetilde{A}}^{0}=\sum_{i \geqslant 0} \partial_{w_{0}}^{i}\left(V^{0} \check{\mathcal{M}}_{\widetilde{A}}^{0} \cap h_{\widetilde{A}, 2, *} F_{p-i}^{H}{ }^{*} \check{\mathcal{M}}_{\widetilde{A}}^{0}\right)
$$

Hence, we need to determine the object $V^{0} \check{\mathcal{M}}_{\widetilde{A}}^{0}$. For what follows, it is more convenient to work out everything at the level of global sections. Since all modules we are considering here are defined on affine spaces, this is obviously sufficient.

We refer to [MM04] for details on the $V$-filtration. For what follows in this introduction, we only need that $V^{0} D_{\mathbb{C}^{3}}=\mathbb{C}\left[w_{0}, w_{1}, w_{2}\right]\left\langle w_{0} \partial_{w_{0}}, \partial_{w_{1}}, \partial_{w_{2}}\right\rangle$ and the following characterization of the canonical $V$-filtration of the holonomic module $\check{M}_{\widetilde{A}}^{0}=\Gamma\left(\mathbb{C}^{3}, \check{\mathcal{M}}_{\widetilde{A}}^{0}\right)$, copied from [MM04, Definition 4.3-3, Proposition 4.3-9]. For any $m \in \check{M}_{\widetilde{A}}^{0}$, we consider its Bernstein-Sato polynomial $b_{m}(x) \in \mathbb{C}[x]$, which is the unique monic polynomial of smallest degree satisfying the functional equation $b_{m}\left(\partial_{w_{0}} w_{0}\right) m \in w_{0} \cdot V^{0}\left(D_{\mathbb{C}^{3}}\right) m$. The set of roots of $b_{m}(x)$ is denoted by $\operatorname{ord}(m)$. Then we have

$$
V^{\alpha} \check{M}_{\widetilde{A}}^{0}:=\left\{m \in \check{M}_{\widetilde{A}}^{0} \mid \operatorname{ord}(m) \subset[\alpha, \infty)\right\} .
$$

Our first step is to compute the Bernstein-Sato polynomial for the class [1] $\in \check{M}_{\widetilde{A}}^{0}$. Here we have the following result. (Compare with Lemma 4.4 for the general case.)

Proposition 2.3. Consider the class of $1 \in D_{\mathbb{C}^{3}}$ in the quotient $\check{M}_{\widetilde{A}}^{0}$, denoted by [1]. Then we have $b_{[1]}(s)=s^{2}$.

Proof. It is sufficient to find a functional equation in $D_{\mathbb{C}^{3}}$ of the form $\left(\partial_{w_{0}} w_{0}\right)^{2}=w_{0} \cdot P+\check{I}_{\widetilde{A}}^{0}$, where

$$
\check{I}_{\widetilde{A}}^{0}:=\left(w_{0}^{2}-w_{1} w_{2}, \partial_{w_{0}} w_{0}+\partial_{w_{1}} w_{1}+\partial_{w_{2}} w_{2}, \partial_{w_{1}} w_{1}-\partial_{w_{2}} w_{2}\right)
$$

and $P \in \mathbb{C}\left[w_{0}, w_{1}, w_{2}\right]\left\langle w_{0} \partial_{w_{0}}, \partial_{w_{1}}, \partial_{w_{2}}\right\rangle$. We will show that

$$
\left(-\partial_{w_{1}} w_{1}-\partial_{w_{2}} w_{2}\right)^{2} \in w_{0} \cdot \mathbb{C}\left[w_{1}, w_{2}\right]\left\langle\partial_{w_{1}}, \partial_{w_{2}}\right\rangle+\check{I}_{\widetilde{A}}^{0},
$$




\section{Hypergeometric Hodge modules}

which suffices to conclude. We have

$$
\begin{aligned}
\left(-\partial_{w_{1}} w_{1}-\partial_{w_{2}} w_{2}\right)^{2} & =2 \partial_{w_{1}} \partial_{w_{2}} w_{1} w_{2}+\left(\partial_{w_{1}} w_{1}\right)^{2}+\left(\partial_{w_{2}} w_{2}\right)^{2} \\
& \equiv 2 w_{0}^{2} \cdot \partial_{w_{1}} \partial_{w_{2}}+\left(\partial_{w_{1}} w_{1}\right)^{2}+\left(\partial_{w_{2}} w_{2}\right)^{2} \bmod \check{I}_{\widetilde{A}}^{0} \\
& =2 w_{0}^{2} \cdot \partial_{w_{1}} \partial_{w_{2}}+\left(\partial_{w_{1}} w_{1}-\partial_{w_{2}} w_{2}\right)^{2}+2 \partial_{w_{1}} \partial_{w_{2}} w_{1} w_{2} \\
& \equiv w_{0}^{2} \cdot 4 \cdot \partial_{w_{1}} \partial_{w_{2}} \bmod \check{I}_{\widetilde{A}}^{0}
\end{aligned}
$$

which shows formula (2.2).

Notice that the above calculation can be extended to any matrix $\widetilde{A}$ of the form

$$
\widetilde{A}=\left(\begin{array}{cccc}
1 & 1 & \ldots & 1 \\
0 & a_{11} & \ldots & a_{1 n} \\
\vdots & \vdots & & \vdots \\
0 & a_{d 1} & \ldots & a_{d n}
\end{array}\right),
$$

where the columns of the matrix

$$
A=\left(\begin{array}{ccc}
a_{11} & \ldots & a_{1 n} \\
\vdots & & \vdots \\
a_{n 1} & \ldots & a_{n n}
\end{array}\right)
$$

are the primitive integral generators of the fan of a smooth projective toric Fano manifold. Then one has to consider the classical cohomology algebra of this manifold, which admits a toric description (see, for example, [Ful93, Section 5.2]), and the functional equation (that is, the analogue of formula (2.2)) can be deduced from the relations in this algebra. Also notice that this is in fact an argument which is a much simplified version of the one used to prove Lemma 4.4 (actually, the proof of this lemma relies on the main result of the separate paper [RSW18], which is based on general arguments from toric algebra, such as Euler-Koszul complexes, toric modules, etc.). Lemma 4.4 is also more general in the sense that the matrix considered there is not necessarily defined by the rays of a smooth toric variety.

We have the following consequence of the above calculation, which gives complete control over the integer part of the canonical $V$-filtration on $\check{M}$. (Compare with Proposition 4.8 for the general case.)

Corollary 2.4. Denote by $V_{\text {ind }}^{\bullet} \check{M}_{\widetilde{A}}^{0}$ the filtration induced on $\check{M}_{\widetilde{A}}^{0}$ by the $V$-filtration (with respect to $\left.w_{0}\right)$ on $D_{\mathbb{C}^{3}}$. Then for all $\stackrel{k}{k} \in \mathbb{Z}$, we have $V_{\text {ind }}^{k} \check{M}_{\widetilde{A}}^{0}=V^{k} \check{M}_{\widetilde{A}}^{0}$.

Proof. The proof is more or less similar to that of the general case in Proposition 4.8. In the case $k \geqslant 0$, any element $[P] \in V_{\text {ind }}^{k} \check{M}_{\widetilde{A}}^{0}$ has an expression

$$
[P]=\left[\sum_{i=0}^{l} w_{0}^{k}\left(w_{0} \partial_{w_{0}}\right)^{i} \cdot P_{i}\right]+[R],
$$

where $P_{i} \in \mathbb{C}\left[w_{1}, w_{2}\right]\left\langle\partial_{w_{1}}, \partial_{w_{2}}\right\rangle$ and $[R] \in V_{\text {ind }}^{k+1} \check{M}_{\widetilde{A}}^{0}$. On the other hand, if $k>0$, we can always write an element $[P] \in V_{\text {ind }}^{-k} \check{M}_{\widetilde{A}}^{0}$ as

$$
[P]=\left[\sum_{i=0}^{l} \partial_{w_{1}}^{k}\left(w_{1} \partial_{w_{1}}\right)^{i} \cdot P_{i}\right]+[R] .
$$




\section{T. Reichelt And C. Sevenheck}

where $P_{i}$ and $[R]$ are as above. One easily deduces (see the calculations in the proof of Proposition 4.8) from the functional equation $\left(\partial_{w_{0}} w_{0}\right)^{2}[1] \in V_{\text {ind }}^{1} \check{M}_{\widetilde{A}}^{0}$ proved in Proposition 2.3 that we have

$$
\begin{aligned}
\left(\partial_{w_{0}} w_{0}-k\right)^{2}[P] \in V_{\text {ind }}^{k+1} \check{M}_{\widetilde{A}}^{0} & \text { for } k \geqslant 0 \text { and }[P] \in V_{\text {ind }}^{k} \check{M}_{\widetilde{A}}^{0}, \\
\left(\partial_{w_{0}} w_{0}+k\right)^{2}[P] \in V_{\text {ind }}^{-k+1} \check{M}_{\widetilde{A}}^{0} & \text { for } k>0 \text { and }[P] \in V_{\text {ind }}^{-k} \check{M}_{\widetilde{A}}^{0} .
\end{aligned}
$$

General considerations on the canonical $V$-filtration (see, for example, [MM04, Sections 4.2 and 4.3] and the argument in the proof of Proposition 4.8) then imply that $V_{\text {ind }}^{k} \check{M}_{\widetilde{A}}^{0}=V^{k} \check{M}_{\widetilde{A}}^{0}$.

Finally, we arrive at the following first main step toward the proof of Theorem 2.1. (Compare with Theorem 4.17 for the general case.)

Proposition 2.5. Let $\widetilde{A}$ and $h_{\widetilde{A}}:\left(\mathbb{C}^{*}\right)^{2} \rightarrow \mathbb{C}^{3}=W$ be as above; then for any $k \in \mathbb{Z}$, we have the following isomorphism of $\mathcal{O}_{W}$-modules:

$$
F_{p}^{H} h_{\widetilde{A},+} \mathcal{O}_{\left(\mathbb{C}^{*}\right)^{2}} \cong F_{p-1}^{\text {ord }}\left[\mathcal{D}_{W} /\left(w_{0}^{2}-w_{1} w_{2}, \partial_{w_{0}} w_{0}+\partial_{w_{1}} w_{1}+\partial_{w_{2}} w_{2}, \partial_{w_{1}} w_{1}-\partial_{w_{2}} w_{2}\right)\right]
$$

Proof. The main tool to obtain a description of the Hodge filtration is formula (2.1) (see [Sai93, Proposition 4.2.]), which at the level of global sections reads

$$
F_{p}^{H} \check{M}_{\widetilde{A}}^{0}=\sum_{i \geqslant 0} \partial_{w_{0}}^{i}\left(V^{0} \check{M}_{\widetilde{A}}^{0} \cap F_{p-i}^{H}{ }^{*} \check{M}_{\widetilde{A}}^{0}\right)
$$

Recall from Lemma 2.2 that for all $l \in \mathbb{Z}$, we have

$$
F_{l}^{H *} \check{M}_{\widetilde{A}}^{0}=F_{l-1}^{\mathrm{ord} *} \check{M}_{\widetilde{A}}^{0} .
$$

In particular, since $F_{p}^{\text {ord } *} \check{M}_{\widetilde{A}}^{0}=0$ for all $p<0$, we have $F_{p}^{H} \check{M}_{\widetilde{A}}^{0}=0$ for all $p<1$. On the other hand, we have seen in Proposition 2.3 that $[1] \in V^{0} \check{M}_{\widetilde{A}}^{0}$. Obviously, we have $[1] \in F_{0}^{\text {ord } *} \check{M}_{\widetilde{A}}^{0}$, which implies that $[1] \in F_{1}^{H} \check{M}_{\widetilde{A}}^{0}$. Since $\check{M}_{\widetilde{A}}^{0}$ is a cyclic $D_{W}$-module and since both filtrations $F^{H}$ and $F^{\text {ord }}$ on it are good filtrations, we obtain the inclusion

$$
F_{p-1}^{\mathrm{ord}} \check{M}_{\widetilde{A}}^{0} \subset F_{p}^{H} \check{M}_{\widetilde{A}}^{0}
$$

for all $p \in \mathbb{Z}$. It remains to show the reverse inclusion $F_{p}^{H} \check{M}_{\widetilde{A}}^{0} \subset F_{p-1}^{\text {ord }} \check{M}_{\widetilde{A}}^{0}$. Using formula (2.3) as well as Corollary 2.4 and Lemma 2.2, this amounts to

$$
\sum_{i \geqslant 0} \partial_{w_{0}}^{i}\left(V_{\text {ind }}^{0} \check{M}_{\widetilde{A}}^{0} \cap F_{p-i}^{\mathrm{ord} *} \check{M}_{\widetilde{A}}^{0}\right) \subset F_{p}^{\mathrm{ord}} \check{M}_{\widetilde{A}}^{0}
$$

We obviously have $\partial_{w_{0}}^{i} F_{p-i}^{\text {ord }} \check{M}_{\widetilde{A}}^{0} \subset F_{p}^{\text {ord }} \check{M}_{\widetilde{A}}^{0}$, for all $i$, so it only remains to show that

$$
V_{\text {ind }}^{0} \check{M}_{\widetilde{A}}^{0} \cap F_{l}^{\text {ord } *} \check{M}_{\widetilde{A}}^{0} \subset F_{l}^{\text {ord }} \check{M}_{\widetilde{A}}^{0}
$$

for all $l \in \mathbb{Z}$. Consider any class $[P] \in V_{\text {ind }}^{0} \check{M}_{\widetilde{A}}^{0} \cap F_{l}^{\text {ord*} *} \check{M}_{\widetilde{A}}^{0}$. Since we have ${ }^{*} \check{M}_{\widetilde{A}}^{0}=\check{M}_{\widetilde{A}}^{0}\left[w_{0}^{-1}\right]$, we can write

$$
P=w_{0}^{-k} P_{k}+w_{0}^{-k+1} P_{-k+1}+\cdots,
$$

where $P_{i} \in \mathbb{C}\left[w_{1}, w_{2}\right]\left\langle\partial_{w_{0}}, \partial_{w_{1}}, \partial_{w_{2}}\right\rangle$. It follows that $w_{0}^{k} \cdot[P] \in V_{\text {ind }}^{k} \check{M}_{\widetilde{A}}^{0} \cap F_{l}^{\text {ord }} \check{M}_{\widetilde{A}}^{0}$. We thus have to prove

$$
V_{\text {ind }}^{k} \check{M}_{\widetilde{A}}^{0} \cap F_{l}^{\text {ord }} \check{M}_{\widetilde{A}}^{0} \subset w_{0}^{k} F_{l}^{\text {ord }} \check{M}_{A}^{0}
$$

for all $k, l \in \mathbb{Z}$. Take any class $[Q] \in V_{\text {ind }}^{k} \check{M}_{\widetilde{A}}^{0} \cap F_{l}^{\text {ord }} \check{M}_{\widetilde{A}}^{0}$; then suppose that we can find a representative $Q \in V^{k} D_{W} \cap F_{l} D_{W}$ of $[Q]$. This means that $Q=w_{0}^{k} \cdot \widetilde{Q}$ with $\widetilde{Q} \in F_{l} D_{W}$, as required. 


\section{Hypergeometric Hodge modules}

Hence, we obtain $[Q] \in w_{0}^{k} F_{l}^{\text {ord }} \check{M}_{\widetilde{A}}^{0}$. It thus remains to show the existence of such a representative $Q \in V^{k} D_{W} \cap F_{l} D_{W}$, and this is exactly the content of the next lemma. (Compare with Proposition 4.9 for the general case.)

Lemma 2.6. Let $A$ be as above; then for all $k, l \in \mathbb{Z}$, the morphism

$$
V^{k} D_{W} \cap F_{l} D_{W} \longrightarrow V_{\text {ind }}^{k} \check{M}_{\widetilde{A}}^{0} \cap F_{l}^{\text {ord }} \check{M}_{\widetilde{A}}^{0}
$$

is surjective.

Proof. The proof relies on the theory of Gröbner bases in the Weyl algebra. We will not give any definition here, but we refer to Subsection 4.1 for details about monomial orders and Gröbner bases in the non-commutative setup.

Consider any class $m \in V_{\text {ind }}^{k} \check{M}_{\widetilde{A}}^{0} \cap F_{l}^{\text {ord }} \check{M}_{\widetilde{A}}^{0}$. Then we can find $P \in F_{l} D_{W}$ and $Q \in V^{k} D_{W}$ such that $[P]=[Q]=m$, that is, $P=Q+i$ for some

$$
i \in \check{I}_{\widetilde{A}}^{0}=\left(w_{0}^{2}-w_{1} w_{2}, \partial_{w_{0}} w_{0}+\partial_{w_{1}} w_{1}+\partial_{w_{2}} w_{2}, \partial_{w_{1}} w_{1}-\partial_{w_{2}} w_{2}\right) .
$$

We choose a minimal $r \in \mathbb{N}$ with $Q \in F_{r} D_{W}$. If $r \leqslant l$, we are done since then $Q \in V^{k} D_{W} \cap F_{l} D_{W}$ is the preimage of $m$ we are looking for. Hence, suppose $r>l$. It is easy to see that then $i \in F_{r} D_{W}$ and the class of $i$ in $\operatorname{Gr}_{r}^{F} D_{W}$ is non-zero. For any operator $R \in D_{W}$, write

$$
\sigma(R) \in \operatorname{Gr}_{\bullet}^{F} D_{W}=\mathbb{C}\left[w_{0}, w_{1}, w_{2}, \xi_{0}, \xi_{1}, \xi_{2}\right]=: \mathbb{C}[\underline{w}, \underline{\xi}]
$$

for its symbol. The three generators of $\check{I}_{\widetilde{A}}^{0}$ from above form a Gröbner basis of this ideal with respect to the partial ordering given by the weight vector $(\underline{0}, \underline{1})$ (that is, where $w_{i}$ has weight 0 and $\partial_{w_{i}}$ has weight 1 ); notice that this weight vector induces the filtration $F_{\bullet}$ on $D_{W}$. This can be directly shown by a Macaulay2 calculation; the corresponding general result is Corollary 4.13, where, however, we treat a slightly different situation. There, we add a column to our matrix which is the sum of all other columns. This corresponds to composing with the graph embedding of the equation of a normal crossing divisor; see also the remarks before Lemma 4.14.

We can therefore conclude that there is an expression

$$
\sigma(i)=\widetilde{i}_{1} \cdot\left(w_{0}^{2}-w_{1} w_{2}\right)+\widetilde{i}_{2} \cdot\left(\xi_{0} w_{0}+\xi_{1} w_{1}+\xi_{2} w_{2}\right)+\widetilde{i}_{3} \cdot\left(\xi_{1} w_{1}-\xi_{2} w_{2}\right)
$$

with $\widetilde{i}_{1}, \widetilde{i}_{2}, \widetilde{i}_{3} \in \mathbb{C}[\underline{w}, \underline{\xi}]$. Let $i_{1}, i_{2}, i_{3} \in D_{W}$ be the normally ordered operators obtained from $\widetilde{i}_{1}$, $\widetilde{i}_{2}, \widetilde{i}_{3}$ by replacing $\xi_{k}$ with $\partial_{w_{k}}$. Then we define

$$
i^{\prime}:=i_{1}\left(w_{0}^{2}-w_{1} w_{2}\right)+i_{2}\left(\partial_{w_{0}} w_{0}+\partial_{w_{1}} w_{1}+\partial_{w_{2}} w_{2}\right)+i_{3}\left(\partial_{w_{1}} w_{1}-\partial_{w_{2}} w_{2}\right) \in \check{I}_{\widetilde{A}}^{0},
$$

and clearly $i^{\prime} \in F_{l} D_{W}$. However, we also have $i^{\prime} \in V^{k} D_{W}$ since it can again be shown by a direct computation that the three polynomials $w_{0}^{2}-w_{1} w_{2}, \xi_{0} w_{0}+\xi_{1} w_{1}+\xi_{2} w_{2}, \xi_{1} w_{1}-\xi_{2} w_{2}$ form a Gröbner basis of the ideal they generate with respect to a partial ordering given by the weight vector $(-1,0,0,1,0,0)$ (that is, the weight of $w_{0}$ is -1 , the weight of $\xi_{0}$ is 1 , and all other weights are zero). Notice again that this weight vector yields the filtration induced from $V^{\bullet} D_{W}$ on $\mathbb{C}[\underline{w}, \xi]$. The corresponding general result (for the case of the extended matrix with one added column) is found in Corollary 4.13(ii).

Summarizing, we obtain that the operator $Q-i^{\prime}$ satisfies

(1) $\left[Q-i^{\prime}\right]=[Q]=m$,

(2) $Q-i^{\prime} \in V^{k} D_{W}$

(3) $Q-i^{\prime} \in F_{l-1} D_{W}$ (this follows from $\sigma(Q)=\sigma(i)=\sigma\left(i^{\prime}\right)$ ). 


\section{T. Reichelt And C. Sevenheck}

Hence, we see by descending induction on $l$ that we can construct an operator $Q^{\prime} \in F_{l} D_{W} \cap V^{k} D_{W}$ such that $\left[Q^{\prime}\right]=m$. This shows the statement.

We have finished the proof of Proposition 2.5, which roughly summarizes the content of Section 4 in the main body of the paper for our particular example. We now turn to the statements corresponding to Section 5 (for our example), that is, we are going to complete the proof of Theorem 2.1.

Recall that the GKZ system $\mathcal{M}_{\widetilde{A}}^{0}$ can be described as a Fourier-Laplace transform of a torus embedding

$$
\mathcal{M}_{\widetilde{A}}^{0} \simeq \mathrm{FL}\left(h_{\widetilde{A},+} \mathcal{O}_{\left(\mathbb{C}^{*}\right)^{2}}\right) .
$$

Since the matrix $\widetilde{A}$ is homogeneous (that is, $(1, \ldots, 1)$ is in its row span), the $\mathcal{D}$-module $\mathcal{M}_{\widetilde{A}}^{0}$ has a different presentation involving only (proper) direct image functors and inverse image functors but excluding the use of the Fourier-Laplace transformation [Rei14, Proposition 2.7(iii)]. Let us recall some ingredients of this construction in the present situation. Consider the torus embedding

$$
g: \mathbb{C}^{*} \longrightarrow \mathbb{P}^{2}, \quad t \longmapsto\left(1: t: t^{-1}\right) ;
$$

then $\mathcal{M}_{\widetilde{A}}^{0}$ can be described by a Radon-type transform of the $\mathcal{D}$-module $g_{+} \mathcal{O}_{\mathbb{C}^{*}}$. More precisely, we have a commutative diagram

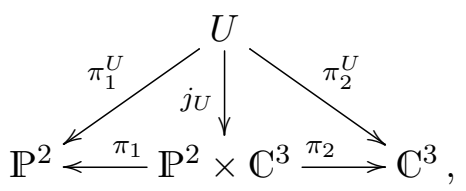

where $U$ is the complement of the universal hyperplane in $\mathbb{P}^{2} \times \mathbb{C}^{3}$; that is, $U:=\left\{\lambda_{0} w_{0}+\lambda_{1} w_{1}+\right.$ $\left.\lambda_{2} w_{2} \neq 0\right\}$. The GKZ system $\mathcal{M}_{\widetilde{A}}^{0}$ is now given by

$$
\mathcal{M}_{\widetilde{A}}^{0} \simeq \mathcal{R}_{c}^{\circ}\left(g_{+} \mathcal{O}_{\mathbb{C}^{*}}\right) \simeq \pi_{2 \dagger}^{U} \pi_{1}^{U, \dagger} g_{+} \mathcal{O}_{\mathbb{C}^{*}} \simeq \pi_{2,+}\left(\pi_{1}^{\dagger} g_{+} \mathcal{O}_{T} \otimes j_{U, \dagger} \mathcal{O}_{U}\right)
$$

where the last isomorphism follows from the projection formula.

Since $\mathcal{O}_{\mathbb{C}^{*}}$ carries a trivial Hodge module structure and since the category of algebraic mixed Hodge modules is stable under the (proper) direct image functor and the (exceptional) inverse image functor, this induces a mixed Hodge module structure on $\mathcal{M}_{\widetilde{A}}^{0}$.

For technical reasons that mainly occur when dealing with the case $\beta \neq 0$ as we do in the main body of this paper, we will pursue a slightly different approach.

Consider the map $F: \mathbb{C}^{*} \times \mathbb{C}^{3} \rightarrow \mathbb{C}$ given by the Laurent polynomial $\lambda_{0}+\lambda_{1} t+\lambda_{2} t^{-1}$, and let $j: \mathbb{C}^{*} \rightarrow \mathbb{C}$ the canonical embedding. Denote by $p$ and $q$ the projections from $\mathbb{C}^{*} \times \mathbb{C}^{3}$ to the second and first factors, respectively. We consider the integral transform of $\mathcal{O}_{\mathbb{C}^{*}}$ from $\mathbb{C}^{*}$ to $\mathbb{C}^{3}$ with kernel $F^{\dagger} j_{\dagger} \mathcal{O}_{\mathbb{C}^{*}}$ and prove in Proposition 5.4 that this integral transform is isomorphic to the GKZ system $\mathcal{M}_{\widetilde{A}}^{0}$ (In the more general case of a non-zero $\widetilde{\beta}=\left(\beta_{0}, \beta\right)$, we would start with $\mathcal{O}_{\mathbb{C}^{*}}^{\beta}$ and use the kernel $\left.F^{\dagger} j_{\dagger} \mathcal{O}_{\mathbb{C}^{*}}^{\beta_{0}}\right)$.

Since this integral transformation preserves the category of mixed Hodge modules, we define a Hodge module structure on the GKZ system by

$$
{ }^{H} \mathcal{M}_{\widetilde{A}}^{0}:=\mathcal{H}^{6}\left(p_{*}\left(q^{* p} \mathbb{C}_{\mathbb{C}^{*}} \otimes F^{*} j_{!}^{p} \mathbb{C}_{\mathbb{C}^{*}}\right)\right) .
$$

In Proposition 5.5, we prove that this approach coincides with the Radon transform for integer $\beta_{0}$.

In order to compute the Hodge filtration on (2.4) explicitly, we have to consider a partial compactification of $\mathbb{C}^{*} \times \mathbb{C}^{3}$ since the projection $p: \mathbb{C}^{*} \times \mathbb{C}^{2}$ is not proper. For this, we use the 


\section{Hypergeometric Hodge modules}

locally closed embedding $g: \mathbb{C}^{*} \rightarrow \mathbb{P}^{2}$. As an intermediate step, we compute the Hodge filtration on the mixed Hodge module

$$
{ }^{H} \mathcal{N}:=(g \times \mathrm{id})_{*}\left(q^{* p} \mathbb{C}_{\mathbb{C}^{*}} \otimes F^{*} j_{!}^{p} \mathbb{C}_{\mathbb{C}^{*}}\right) .
$$

The space $\mathbb{P}^{2} \times \mathbb{C}^{3}$ is covered by the three charts $W_{u}=\left\{w_{u} \neq 0\right\}$ for $u=0,1,2$. The map $g$ factors over each chart and is given by

$$
\begin{aligned}
& g_{0}: \mathbb{C}^{*} \longrightarrow W_{0}, \quad g_{1}: \mathbb{C}^{*} \longrightarrow W_{1}, \quad g_{2}: \mathbb{C}^{*} \longrightarrow W_{2}, \\
& t \longmapsto\left(t, t^{-1}\right), \quad t \longmapsto\left(t^{-1}, t^{-2}\right), \quad t \longmapsto\left(t, t^{2}\right) \text {. }
\end{aligned}
$$

We obtain in formula (5.8) that the restriction of ${ }^{H} \mathcal{N}$ to $W_{u}$ can be written as a direct product

$$
{ }^{H} \mathcal{N}_{u}=\mathcal{H}^{0}\left({ }^{p} g_{u *} \mathbb{C}_{\mathbb{C}^{*}}^{H}\right) \otimes \mathcal{H}^{2}\left(\operatorname{pr}_{u} j^{p}{ }^{p} \mathbb{C}_{\mathbb{C} *}^{H}\right),
$$

where $\operatorname{pr}_{u}: \mathbb{C}^{3} \rightarrow \mathbb{C}$ is the projection to the $(u+1)$ th factor. The Hodge filtration on the first factor can be computed by using Theorem 4.17; the computation of the second factor is straightforward (cf. Remark 4.18) (we check the assumption of Theorem 4.17 in Lemma 5.8).

Define the matrices

$$
A_{0}^{s}:=\left(\begin{array}{ccccc}
0 & 0 & 1 & 1 & 1 \\
1 & -1 & 0 & 1 & -1
\end{array}\right), \quad A_{1}^{s}:=\left(\begin{array}{ccccc}
0 & 0 & 1 & 1 & 1 \\
-1 & -2 & 0 & 1 & -1
\end{array}\right), \quad A_{2}^{s}:=\left(\begin{array}{ccccc}
0 & 0 & 1 & 1 & 1 \\
1 & 2 & 0 & 1 & -1
\end{array}\right) .
$$

We show in Lemma 5.13 that the $\mathcal{D}$-module underlying ${ }^{H} \mathcal{N}_{u}$ is isomorphic to a partial Laplace transformation of $\mathcal{M}_{A_{u}^{s}}^{0}$ in the $w$-variables. More precisely, we have

$$
\mathcal{N}_{0}=\mathcal{D}_{\mathbb{C}^{2} \times \mathbb{C}^{3}} / \stackrel{(\mathcal{I})}{\mathcal{I}}_{A_{0}^{s}}, \quad \mathcal{N}_{1}=\mathcal{D}_{\mathbb{C}^{2} \times \mathbb{C}^{3}} / \stackrel{(\mathcal{I}}{\mathcal{I}}_{A_{1}^{s}}, \quad \mathcal{N}_{2}=\mathcal{D}_{\mathbb{C}^{2} \times \mathbb{C}^{3}} / \stackrel{(\mathcal{I})}{\mathcal{I}}_{A_{2}^{s}}
$$

where $\stackrel{(\mathcal{I})}{\mathcal{I}}_{A_{0}^{s}}$ is generated by the Euler operators

$$
\stackrel{(\vee)}{E_{0}^{0}}:=\lambda_{0} \partial_{\lambda_{0}}+\lambda_{1} \partial_{\lambda_{1}}+\lambda_{2} \partial_{\lambda_{2}}, \quad \stackrel{(\vee)}{E_{1}^{0}}:=-w_{10} \partial_{w_{10}}+w_{20} \partial_{w_{20}}+\lambda_{1} \partial_{\lambda_{1}}-\lambda_{2} \partial_{\lambda_{2}}
$$

and the box operators

$$
\stackrel{(\vee)}{\square}_{(1,1,0,0,0)}:=w_{10} w_{20}-1, \quad \stackrel{(\vee)}{\square}_{(0,0,2,-1,-1)}:=\partial_{\lambda_{0}}^{2}-\partial_{\lambda_{1}} \partial_{\lambda_{2}}, \quad \stackrel{(\vee)}{\square}_{(0,1,-1,1,0)}:=w_{20} \partial_{\lambda_{1}}-\partial_{\lambda_{0}} .
$$

The ideal $\stackrel{(\vee)}{\mathcal{I}}_{A_{1}^{s}}$ is generated by the Euler operators

$$
\stackrel{(\vee)}{E_{0}^{1}}:=\lambda_{0} \partial_{\lambda_{0}}+\lambda_{1} \partial_{\lambda_{1}}+\lambda_{2} \partial_{\lambda_{2}}, \quad \stackrel{(\vee)}{E_{1}^{1}}:=w_{01} \partial_{w_{01}}+2 w_{21} \partial_{w_{21}}+\lambda_{1} \partial_{\lambda_{1}}-\lambda_{2} \partial_{\lambda_{2}}
$$

and the box operators

$$
\stackrel{(\vee V}{\square}_{(2,-1,0,0,0)}:=w_{01}^{2}-w_{21}, \quad \stackrel{(\vee v)}{\square}_{(0,0,2,-1,-1)}:=\partial_{\lambda_{0}}^{2}-\partial_{\lambda_{1}} \partial_{\lambda_{2}}, \quad \stackrel{(\vee V}{\square}_{(1,0,-1,1,0)}:=w_{01} \partial_{\lambda_{1}}-\partial_{\lambda_{0}} .
$$

The ideal $\stackrel{(\vee)}{\mathcal{I}}_{A_{2}^{s}}$ is generated by Euler operators

$$
\stackrel{(\vee)}{E_{0}^{2}}:=\lambda_{0} \partial_{\lambda_{0}}+\lambda_{1} \partial_{\lambda_{1}}+\lambda_{2} \partial_{\lambda_{2}}, \quad \stackrel{(\vee)}{E_{1}^{2}}:=-w_{02} \partial_{w_{01}}-2 w_{12} \partial_{w_{21}}+\lambda_{1} \partial_{\lambda_{1}}-\lambda_{2} \partial_{\lambda_{2}}
$$

and box operators

$$
\stackrel{(\vee v)}{\square}_{(2,-1,0,0,0)}:=w_{02}^{2}-w_{12}, \quad \stackrel{(\vee)}{\square}_{(0,0,2,-1,-1)}:=\partial_{\lambda_{0}}^{2}-\partial_{\lambda_{1}} \partial_{\lambda_{2}}, \quad \stackrel{(\vee v}{\square}_{(1,0,1,-1,0)}:=w_{02} \partial_{\lambda_{0}}-\partial_{\lambda_{1}} .
$$

The Hodge filtration on these systems is given by $F_{p+1}^{H} \mathcal{N}_{u}=F_{p}^{\text {ord }} \mathcal{D}_{W_{u} \times \mathbb{C}^{3}} / \stackrel{(\mathfrak{V})}{\mathcal{I}_{A_{u}^{s}}^{s}}$. 


\section{T. Reichelt And C. Sevenheck}

Using the fact that $\mathcal{N}_{u}$ is a partial Fourier-Laplace transform of $\mathcal{M}_{A_{u}^{s}}$, we use the results of Subsection 3.1 to construct a strict resolution of $\left(\mathcal{N}_{u}, F_{\bullet}^{H}\right)$ at the level of global sections which is given by the Euler-Koszul complex

$$
\stackrel{(\vee)}{K_{u}^{\bullet}}:=D_{W_{u} \times \mathbb{C}^{3}} / \stackrel{(\vee)}{J_{A_{u}^{s}}} \stackrel{\cdot\left(-\stackrel{(\vee)}{E_{1}^{u}}, \stackrel{(\vee \vee}{E_{0}^{u}}\right)^{t}}{\longrightarrow}\left(D_{W_{u} \times \mathbb{C}^{3}} / \stackrel{(\vee)}{J_{A_{u}^{s}}^{s}}\right)^{2} \stackrel{\cdot\left(\stackrel{(\vee)}{E_{0}^{u}}, \stackrel{(\vee)}{\left.E_{1}^{u}\right)}\right)}{\longrightarrow} D_{W_{u} \times \mathbb{C}^{3}} / \stackrel{(\vee)}{J_{A_{u}^{s}}},
$$

where the left ideal $J_{A_{u}^{s}}$ is generated by the "box-type" generators from above.

It remains to compute the projection of $\left(\mathcal{N}, F_{\bullet}^{H}\right)$ under the map $\mathbb{P}^{2} \times \mathbb{C}^{3} \rightarrow \mathbb{C}^{3}$. In order to do this, we lift the filtered $D$-modules $\left(N_{u}, F_{\bullet}^{H}\right)$ as well as their strictly filtered resolution

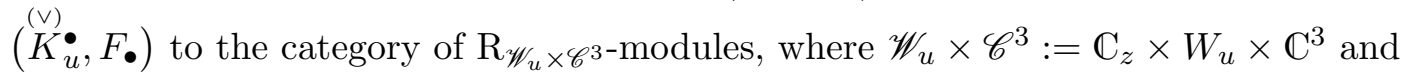

$$
\mathrm{R}_{\mathscr{W}_{u} \times \mathscr{C}^{3}}=\mathbb{C}\left[z,\left(w_{i u}\right)_{i \neq u}, \lambda_{0}, \lambda_{1}, \lambda_{2}\right]\left\langle\left(z \partial_{w_{i u}}\right)_{i \neq u}, z \partial_{\lambda_{0}}, z \partial_{\lambda_{1}}, z \partial_{\lambda_{2}}\right\rangle .
$$

This is done through the Rees construction; that is, we associate with the filtered $D_{W_{U} \times \mathbb{C}^{3-}}$ modules $\left(N_{u}, F_{\bullet}^{H}\right)$ and $\left(\stackrel{(V)}{K}_{u}^{\bullet}, F_{\bullet}\right)$ the $\mathrm{R}_{\mathscr{W}_{u} \times \mathscr{C}^{3} \text {-modules }}$

$$
\mathrm{N}_{u}:=R_{F} N_{u}=\bigoplus_{p \in \mathbb{Z}} F_{p} N_{u} z^{p} \quad \text { and } \quad \mathrm{K}_{u}^{\bullet}:=R_{F} \stackrel{(\mathrm{v})}{K} \stackrel{\bullet}{u},
$$

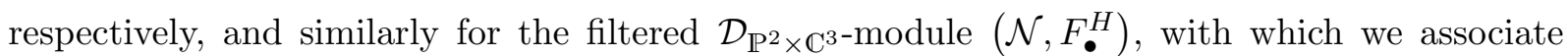
$\mathscr{N}:=\mathcal{O}_{\mathscr{P} \times \mathscr{C}^{3}} \otimes_{\mathcal{O}_{\mathbb{P}^{2} \times \mathbb{C}^{3}}[z]} R_{F^{H}} \mathcal{N}$, where $\mathscr{P} \times \mathscr{C}^{3}:=\mathbb{C}_{z} \times \mathbb{P}^{2} \times \mathbb{C}^{3}$.

Instead of computing the projection of the filtered $\mathcal{D}$-module $\left(\mathcal{N}, F_{\bullet}^{H}\right)$, we compute the projection of the $\mathscr{R}$-module $\mathscr{N}$. This is given by

$$
\pi_{2+\mathscr{N}} \simeq R \pi_{2 *} D R_{\mathscr{P} \times \mathscr{C}^{3} / \mathscr{C}^{3}}(\mathscr{N}),
$$

where this time $\pi_{2}$ denotes the map $\mathscr{P} \times \mathscr{C}^{3} \rightarrow \mathscr{C}^{3}$.

Since this is hard to compute directly, we construct a resolution $\mathscr{K}^{\bullet}$ from the local resolutions $\mathrm{K}_{u}^{\bullet}$ and get the double complex $\Omega_{\mathscr{P} \times \mathscr{C}^{3} / \mathscr{C}^{3}}^{\bullet+2} \otimes \mathscr{K}^{\bullet}$ :

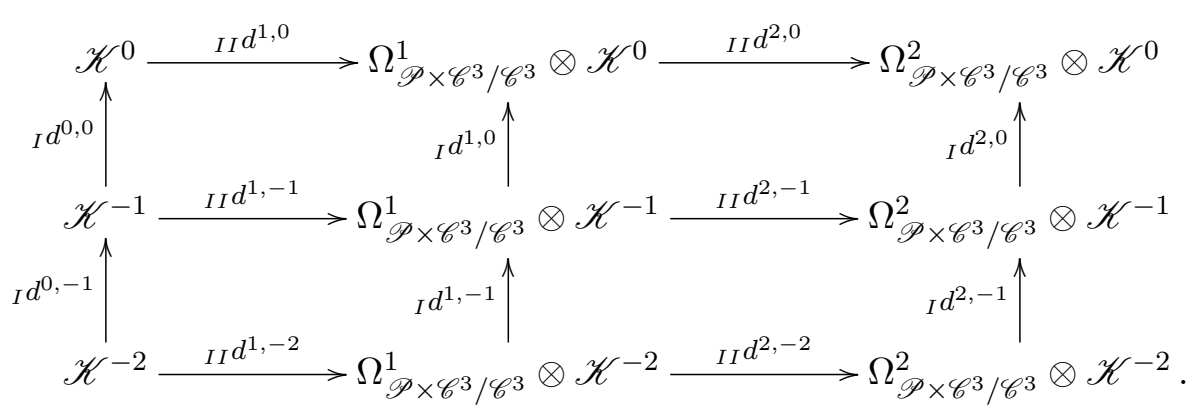

This double complex gives rise to two spectral sequences: The first one is given by first taking cohomology in the vertical direction. This gives the ${ }_{I} E_{1}$-page where only the ${ }_{I} E_{1}^{0, q}$-terms are non-zero; they are isomorphic to $\Omega_{\mathscr{P} \times \mathscr{C}^{3} / \mathscr{C}^{3}}^{q+2} \otimes \mathcal{N}$. If we consider the second spectral sequence and take cohomology in the horizontal direction, we get the ${ }_{I I} E_{1}$-page. Here ${ }_{I I} E_{1}^{p, q}=0$ for $q \neq 0$, and we set $\mathcal{L}^{\bullet}:={ }_{I I} E_{1}^{p, 0}$. Since both spectral sequences degenerate at the second page, we get a quasi-isomorphism $\Omega_{\mathscr{P} \times \mathscr{C}^{3} / \mathscr{C}^{3}}^{\bullet+2} \otimes \mathcal{N} \simeq \mathcal{L}^{\bullet}$ (cf. Proposition 5.20).

In order to get an explicit representation of $\mathcal{L}^{\bullet}$, we introduce a sheaf of rings $\mathscr{S}$ on $\mathscr{P} \times \mathscr{C}^{3}$ and an ideal $\mathscr{J} \subset \mathscr{S}$, which are locally given by

$$
\Gamma\left(\mathscr{W}_{u} \times \mathscr{C}^{3}, \mathscr{S}\right):=S_{\mathscr{W}_{u} \times \mathscr{C}^{3}}:=\mathbb{C}\left[z, \lambda_{0}, \lambda_{1}, \lambda_{2},\left(w_{i u}\right)_{i \neq u}\right]\left\langle z \partial_{\lambda_{0}}, z \partial_{\lambda_{1}}, z \partial_{\lambda_{2}}\right\rangle,
$$




\section{Hypergeometric Hodge modules}

and

$$
\Gamma\left(\mathscr{W}_{u} \times \mathscr{C}^{3}, \mathscr{J}\right)=J_{A_{u}^{s}},
$$

where $J_{A_{u}^{s}}$ is the left ideal in $S_{\mathscr{W}_{u} \times \mathscr{C}^{3}}$ generated by the corresponding box operators.

Define the Euler operators

$$
\tilde{E}_{0}=\lambda_{0} z \partial_{\lambda_{0}}+\lambda_{1} z \partial_{\lambda_{1}}+\lambda_{2} z \partial_{\lambda_{2}}, \quad \tilde{E}_{1}=\lambda_{1} z \partial_{\lambda_{1}}-\lambda_{2} z \partial_{\lambda_{2}} .
$$

We get the quasi-isomorphism

$$
\mathcal{L}^{\bullet} \simeq \operatorname{Kos}^{\bullet}\left(z^{-1} \mathscr{S} / \mathscr{J},\left(\tilde{E}_{k}\right)_{k=0,1}\right)
$$

hence, we get $\pi_{2+} \mathscr{N} \simeq R \pi_{2 *}\left(\operatorname{Kos}^{\bullet}\left(z^{-1} \mathscr{S} / \mathscr{J},\left(\tilde{E}_{k}\right)_{k=0,1}\right)\right)$.

Since $\mathscr{C}^{3}=\mathbb{C}_{z} \times \mathbb{C}^{3}$ is affine, it suffices to compute the global sections of $\pi_{2+} \mathscr{N}$, which are given by

$$
R \Gamma\left(\operatorname{Kos}^{\bullet}\left(z^{-1} \mathscr{S} / \mathscr{J},\left(\tilde{E}_{k}\right)_{k=0,1}\right)\right) .
$$

We will show that each term in the Koszul complex is $\Gamma$-acyclic, which boils down to the fact that $\mathscr{S} / \mathscr{J}$ is $\Gamma$-acyclic.

Define the matrix

$$
A^{s}:=\left(\begin{array}{cccccc}
1 & 1 & 1 & 0 & 0 & 0 \\
0 & 0 & 0 & 1 & 1 & 1 \\
0 & -1 & -1 & 0 & -1 & -1
\end{array}\right)
$$

and the ring

$$
\mathrm{S}:=\mathbb{C}\left[z, w_{0}, w_{1}, w_{2}, \lambda_{0}, \lambda_{1}, \lambda_{2}\right]\left\langle z \partial_{\lambda_{0}}, z \partial_{\lambda_{1}}, z \partial_{\lambda_{2}}\right\rangle .
$$

Let $J_{A^{s}}$ be the left ideal in $S$ generated by

$$
w_{0}^{2}-w_{1} w_{2}, \quad \partial_{\lambda_{0}}^{2}-\partial_{\lambda_{1}} \partial_{\lambda_{2}}, \quad w_{1} \partial_{\lambda_{1}}-w_{0} \partial_{\lambda_{0}}, \quad w_{2} \partial_{\lambda_{2}}-w_{0} \partial_{\lambda_{0}}
$$

(these are box operators with respect to the matrix $A^{s}$ ). The associated sheaf $\widetilde{S / J_{A^{s}}}$ is isomorphic to $\mathscr{S} / \mathscr{J}$. The associated graded module is defined by

$$
\Gamma_{*}(\mathscr{S} / \mathscr{J}):=\bigoplus_{a \in \mathbb{Z}} \Gamma\left(\mathscr{P} \times \mathscr{C}^{3},(\mathscr{S} / \mathscr{J})(a)\right) .
$$

The difference between $S / J_{A^{s}}$ and the associated graded module is measured by local cohomology modules of $S / J_{A^{s}}$; more precisely, we have (cf. Proposition 5.22)

$$
0 \longrightarrow H_{(\underline{w})}^{0}\left(S / J_{A^{s}}\right) \longrightarrow S / J_{A^{s}} \longrightarrow \Gamma_{*}(\mathscr{S} / \mathscr{J}) \longrightarrow H_{(\underline{w})}^{1}\left(S / J_{A^{s}}\right) \longrightarrow 0
$$

and

$$
\bigoplus_{a \in \mathbb{Z}} H^{i}\left(\mathscr{P} \times \mathscr{C}^{3},(\mathscr{S} / \mathscr{J})(a)\right) \simeq H_{(\underline{w})}^{i+1}\left(S / J_{A^{s}}\right)
$$

where $(\underline{w})$ is the ideal in $\mathbb{C}\left[z, w_{0}, w_{1}, w_{2}, \lambda_{0}, \lambda_{1}, \lambda_{2}\right]$ generated by $w_{0}, w_{1}, w_{2}$. Notice that all terms involved carry a natural $\mathbb{Z}$-grading when we $\operatorname{set} \operatorname{deg}\left(w_{i}\right)=1$ and $\operatorname{deg}\left(\lambda_{i}\right)=\operatorname{deg}\left(\partial_{\lambda_{i}}\right)=0$ for $i=$ $0,1,2$. The generators of $J_{A^{s}}$ lie in the commutative subring $T:=\mathbb{C}\left[w_{0}, w_{1}, w_{2}, \partial_{\lambda_{0}}, \partial_{\lambda_{1}}, \partial_{\lambda_{2}}\right] \subset S$. We denote by $K_{A^{s}}$ the corresponding ideal in $T$. It is easily seen that the ring $T / K_{A^{s}}$ is isomorphic to the semigroup ring $\mathbb{C}\left[\mathbb{N} A^{s}\right]$.

We prove that the local cohomology modules turning up in formula (2.5) can be rewritten as follows (cf. Lemma 5.24):

$$
H_{(\underline{w})}^{k}\left(S / J_{A^{s}}\right) \simeq S \otimes_{T} H_{I}^{k}\left(\mathbb{C}\left[\mathbb{N} A^{s}\right]\right),
$$




\section{T. Reichelt And C. Sevenheck}

where the ideal $I \subset \mathbb{C}\left[\mathbb{N} A^{s}\right]$ is generated by $w_{0}, w_{1}, w_{2}$. Hence, we have reduced the problem to a well-known subject in commutative algebra since the local cohomology groups $H_{I}^{k}\left(\mathbb{C}\left[\mathbb{N} A^{s}\right]\right)$ can be explicitly computed by the so-called Ishida complex. Let $\sigma$ be the face which is generated by the first three columns of $A^{s}$ (the columns which correspond to the variables $w_{0}, w_{1}, w_{2}$ ). For a face $\tau \subseteq \sigma$, we define the localization $\mathbb{C}\left[\mathbb{N} A^{s}\right]_{\tau}:=\mathbb{C}\left[\mathbb{N} A^{s}+\mathbb{Z}\left(A^{s} \cap \tau\right)\right]$. Put

$$
L_{\tau}^{k}:=\bigoplus_{\substack{\tau \subset \sigma \\ \operatorname{dim} \tau=k}} \mathbb{C}\left[\mathbb{N} A^{s}\right]_{\tau} .
$$

The Ishida complex therefore takes the form

$$
L_{\sigma}^{\bullet}: 0 \longrightarrow L_{\sigma}^{0} \longrightarrow L_{\sigma}^{1} \longrightarrow L_{\sigma}^{2} \longrightarrow 0 .
$$

We prove, in Proposition 5.25, that $H_{I}^{k}\left(\mathbb{C}\left[\mathbb{N} A^{s}\right]\right) \simeq H^{k}\left(L_{\sigma}^{\bullet}\right)$. Finally, we show in Corollary 5.31 that $H^{k}\left(L_{\sigma}^{\bullet}\right)=0$ for $k \neq 2$ (so we have local cohomology only in the top degree) and that the $\mathbb{Z}$-degrees in $H^{2}\left(L_{\sigma}^{\bullet}\right)$ are purely negative. We refer the reader to Example 5.27 for more details in this particular case.

We can therefore conclude that

$$
\left.S / J_{A^{s}} \simeq \Gamma_{*}(\mathscr{S} / \mathscr{J}) \text { and } H^{i}\left(\mathscr{P} \times \mathscr{C}^{3}, \mathscr{S} / \mathscr{J}\right)\right)=0 \text { for all } i \geqslant 1
$$

Putting things together, we conclude that the global sections of $\pi_{2+} \mathcal{N}$ are given by the module $\Gamma\left(\operatorname{Kos}^{\bullet}\left(z^{-1} \mathscr{S} / \mathscr{J},\left(\tilde{E}_{k}\right)_{k=0,1}\right)\right)$. The latter can be easily computed and gives

$$
\Gamma \mathcal{H}^{0} \pi_{2+} \mathcal{N}=z^{-1} R_{\mathscr{C}^{3}} / I_{\widetilde{A}}^{\lambda} \text { and } \Gamma \mathcal{H}^{i} \pi_{2+} \mathcal{N}=0 \text { for } i \geqslant 1,
$$

where $R_{\mathscr{C}^{3}}:=\mathbb{C}\left[z, \lambda_{0}, \lambda_{1}, \lambda_{2}\right]\left\langle z \partial_{\lambda_{0}}, z \partial_{\lambda_{1}}, z \partial_{\lambda_{2}}\right\rangle$ and the left ideal $I_{\widetilde{A}}^{\lambda}$ is generated by the box operator $\left(z \partial_{\lambda_{0}}\right)^{2}-\left(z \partial_{\lambda_{1}}\right)\left(z \partial_{\lambda_{2}}\right)$ and the Euler operators $\tilde{E}_{0}, \tilde{E}_{1}$. But this shows that

$$
\left(\mathcal{M}_{\widetilde{A}}^{0}, F_{\bullet}^{H}\right) \simeq\left(\mathcal{M}_{\widetilde{A}}^{0}, F_{\bullet+1}^{\text {ord }}\right)
$$

which is the statement of Theorem 2.1 (or that of Theorem 5.35 in the general case).

\section{GKZ systems and the Fourier-Laplace transform}

We start by introducing GKZ systems as well as their Fourier-Laplace transformed versions. Throughout the paper, we let $W$ be a finite-dimensional vector space over $\mathbb{C}$ and denote its dual vector space by $V$. We will fix coordinates $w_{1}, \ldots, w_{n}$ on $W$ and dual coordinates $\lambda_{1}, \ldots, \lambda_{n}$ on $V$.

\subsection{GKZ systems and strict resolutions}

Given a $d \times n$ integer matrix $A=\left(a_{k i}\right)$, we denote its columns by $\underline{a}_{1}, \ldots, \underline{a}_{n}$. We define

$$
\mathbb{N} A:=\sum_{i=1}^{n} \mathbb{N} \underline{a}_{i}
$$

and similarly for $\mathbb{Z} A$ and $\mathbb{R}_{\geqslant 0} A$. Throughout the paper, we assume that the matrix $A$ satisfies

$$
\mathbb{Z} A=\mathbb{Z}^{d} \text {. }
$$

Definition 3.1. Let $A=\left(a_{k i}\right)$ be a $d \times n$ integer matrix with $\mathbb{Z} A=\mathbb{Z}^{d}$ and $\beta=\left(\beta_{1}, \ldots, \beta_{d}\right)$ $\in \mathbb{C}^{d}$. Write $\mathbb{L}_{A}$ for the $\mathbb{Z}$-module of integer relations among the columns of $A$, and write $\mathcal{D}_{V}$ for 


\section{HypergeOMetric Hodge Modules}

the sheaf of rings of differential operators on $V$. Define

$$
\mathcal{M}_{A}^{\beta}:=\mathcal{D}_{V} / \mathcal{I}_{A}
$$

where $\mathcal{I}_{A}$ is the sheaf of left ideals generated by

$$
\square_{\underline{l}}:=\prod_{i: l_{i}<0} \partial_{\lambda_{i}}^{-l_{i}}-\prod_{i: l_{i}>0} \partial_{\lambda_{i}}^{l_{i}}
$$

for all $\underline{l} \in \mathbb{L}_{A}$ and

$$
E_{k}-\beta_{k}:=\sum_{i=1}^{n} a_{k i} \lambda_{i} \partial_{\lambda_{i}}-\beta_{k}
$$

for $k=1, \ldots, d$.

Since GKZ systems are defined on the affine space $V \cong \mathbb{C}^{n}$, we will often work with the $D$-modules of global sections $M_{A}^{\beta}:=\Gamma\left(\mathbb{C}^{n}, \mathcal{M}_{A}^{\beta}\right)$ rather than with the sheaves themselves.

We will now discuss filtrations on GKZ systems given by a weight vector $(u, v) \in \mathbb{Z}^{2 n}$. This weight vector induces an increasing filtration on $D_{V}$ given by

$$
F_{p}^{(u, v)} D_{V}=\left\{\sum_{\substack{\sum_{i} u_{i} \gamma_{i}+v_{i} \delta_{i} \leqslant p \\ \text { finite }}} c_{\gamma \delta} \lambda^{\gamma} \partial_{\lambda}^{\delta} \mid \gamma, \delta \in \mathbb{Z}_{\geqslant 0}^{n}\right\},
$$

where we set $\lambda^{\gamma}:=\prod_{i=1}^{n} \lambda_{i}^{\gamma_{i}}$, etc. For an element $P=\sum c_{\gamma \delta} \lambda^{\gamma} \partial_{\lambda}^{\delta}$, we define

$$
\operatorname{ord}_{(u, v)}(P):=\max \left\{\sum_{i} u_{i} \gamma_{i}+v_{i} \delta_{i} \mid c_{\gamma \delta} \neq 0\right\} .
$$

The associated graded ring $\mathrm{Gr}_{\bullet}^{(u, v)} D_{V}$ is given by $\bigoplus_{p} F_{p}^{(u, v)} D_{V} / F_{p-1}^{(u, v)} D_{V}$.

In order to construct a strictly filtered resolution of $\mathcal{M}_{A}^{\beta}$, we use the theory of Euler-Koszul complexes as introduced in [MMW05]. We will work at the level of global sections. We briefly recall the definition of the Euler-Koszul complex $\left(K^{\bullet}, E-\beta\right)$ from [MMW05, Definition 4.2] (where it is called $\mathcal{K}_{\bullet}(E-\beta ; \mathbb{C}[\mathbb{N} A])$ and placed in positive homological degrees). Its terms are given by

$$
K^{-l}=\bigoplus_{0 \leqslant i_{1}<\cdots<i_{l} \leqslant l}\left(D_{V} / J_{A}\right) e_{i_{1} \ldots i_{l}},
$$

where the left ideal $J_{A} \subset \mathbb{C}[\underline{\partial}]:=\mathbb{C}\left[\partial_{\lambda_{1}}, \ldots, \partial_{\lambda_{n}}\right]$ is generated by

$$
\square_{\underline{l}}:=\prod_{i: l_{i}<0} \partial_{\lambda_{i}}^{-l_{i}}-\prod_{i: l_{i}>0} \partial_{\lambda_{i}}^{l_{i}}, \quad \forall \underline{l} \in \mathbb{L}_{A} .
$$

A simple computation using the fact that $\sum_{i=1}^{n} l_{i} a_{k i}=0$ shows that the maps

$$
D_{V} / D_{V} J_{A} \longrightarrow D_{V} / D_{V} J_{A}, \quad P \mapsto P \cdot\left(E_{k}-\beta_{k}\right) \quad \text { for } \quad k=1, \ldots, d
$$

are well defined. Moreover, we have $\left[E_{k_{1}}-\beta_{k_{1}}, E_{k_{2}}-\beta_{k_{2}}\right]=0$ for $k_{1}, k_{2} \in\{1, \ldots, d\}$, and hence we can build the Koszul complex

$$
\left(K^{\bullet}, E-\beta\right)=\left(\cdots \stackrel{d_{-2}}{\longrightarrow} K^{-1} \stackrel{d_{-1}}{\longrightarrow} K^{0} \rightarrow 0\right):=\operatorname{Kos}\left(D_{V} / D_{V} J_{A},\left(E_{k}-\beta_{k}\right)_{1=0, \ldots, d}\right)
$$

with $D_{V}$-linear differential

$$
d_{-l}\left(e_{i_{1} \ldots i_{l}}\right):=\sum_{k=1}^{l}(-1)^{l-1}\left(E_{i_{k}}-\beta_{i_{k}}\right) e_{i_{1} \ldots \hat{i}_{k} \ldots i_{l}} .
$$




\section{T. Reichelt And C. Sevenheck}

If we assume that the semigroup $\mathbb{N} A$ satisfies $\mathbb{N} A=\mathbb{Z}^{d} \cap \mathbb{R}_{\geqslant_{0}} A$, then by a classical result due to Hochster [Hoc72, Theorem 1], it follows that the semigroup ring $\mathbb{C}[\mathbb{N} A]$ is Cohen-Macaulay. It was shown in [MMW05, Remark 6.4] that in this case $\left(K^{\bullet}, E-\beta\right)$ is a resolution of $M_{A}^{\beta}$ for all $\beta \in \mathbb{C}^{d}$.

Notice that the filtration $F_{\bullet}^{(u, v)}$ on $D_{V}$ induces a filtration on $D_{V} / D_{V} J_{A}$; we denote it by the same symbol. We define the following filtration on each term of the Koszul complex $\left(K^{\bullet}, E-\beta\right)$ :

$$
F_{p}^{(u, v)} K^{-l}:=\bigoplus_{0 \leqslant i_{1}<\cdots<i_{l} \leqslant l} F_{p-\sum_{k=1}^{l} c_{i_{k}}}^{(u, v)}\left(D_{V} / D_{V} J_{A}\right) e_{i_{1} \ldots i_{i_{l}}}
$$

where $c_{i}=\operatorname{ord}_{(u, v)}\left(E_{i}-\beta_{i}\right)$. This shows that the complex $\left(\left(K^{\bullet}, E-\beta\right), F_{\bullet}^{(u, v)}\right)$ is filtered, in other words, that the differential $d$ respects the filtration

$$
d_{-l}\left(F_{p}^{(u, v)} K^{-l}\right) \subset F_{p}^{(u, v)} d_{-l}\left(K^{-l}\right):=\operatorname{im}\left(d_{-l}\right) \cap F_{p}^{(u, v)} K^{-l+1} .
$$

We recall the following well-known criterion for a complex to be strictly filtered, which means

$$
d_{-l}\left(F_{p}^{(u, v)} K^{-l}\right)=F_{p}^{(u, v)} d_{-l}\left(K^{-l}\right)=\operatorname{im}\left(d_{-l}\right) \cap F_{p}^{(u, v)} K^{-l+1} .
$$

LEMMA 3.2. Let

$$
0 \longrightarrow\left(M_{1}, F\right) \stackrel{d_{1}}{\longrightarrow} \cdots \stackrel{d_{n-1}}{\longrightarrow}\left(M_{n}, F\right) \longrightarrow 0
$$

be a sequence of filtered $D$-modules with bounded below filtration. The following properties are equivalent:

(i) The $\operatorname{map} d_{k}$ is strict.

(ii) $H^{k}\left(F_{p} M_{\bullet}\right) \simeq F_{p} H^{k}\left(M_{\bullet}\right)$ for all $p$.

(iii) $H^{k}\left(\operatorname{Gr}_{p}^{F} M_{\bullet}\right) \simeq \operatorname{Gr}_{p}^{F} H^{k}\left(M_{\bullet}\right)$ for all $p$.

Remark 3.3. Suppose that we have $\mathbb{N} A=\mathbb{Z}^{d} \cap \mathbb{R}_{\geqslant 0} A$; then in order to prove that the filtered complex $\left(\left(K^{\bullet}, E-\beta\right), F_{\bullet}^{(u, v)}\right)$ is strict, it is enough to show that $H^{-l}\left(\operatorname{Gr}_{\bullet}^{(u, v)} K^{\bullet}\right)=0$ for $l>1$ and $H^{0}\left(\operatorname{Gr}_{\bullet}^{(u, v)} K^{\bullet}\right)=\operatorname{Gr}_{\bullet}^{(u, v)} M_{A}^{\beta}$ since we already know that $H^{-l}\left(K^{\bullet}\right)=0$ for $l>1$ and $H^{0}\left(K^{\bullet}\right)=M_{A}^{\beta}$.

\subsection{Fourier-Laplace transformed GKZ systems}

Let as before $W$ be an $n$-dimensional vector space over $\mathbb{C}$, and denote its dual vector space by $V$. Let $X$ be a smooth algebraic variety and $E=X \times W$ be a trivial vector bundle and $E^{\prime}:=X \times V$ its dual. We write $\langle\rangle:, W \times V \rightarrow \mathbb{C}$ for the canonical pairing which extends to a function $\langle\rangle:, E \times E^{\prime} \rightarrow \mathbb{C}$.

Definition 3.4. Define $\mathcal{L}:=\mathcal{O}_{E \times_{X} E^{\prime}} e^{-\langle,\rangle}$, which is by definition the free rank one module with differential given by the product rule. Denote the canonical projections by $p_{1}: E \times_{X} E^{\prime} \rightarrow E$ and $p_{2}: E \times_{X} E \rightarrow E^{\prime}$. For $\mathcal{M} \in D_{h}^{b}\left(\mathcal{D}_{E}\right)$, the Fourier-Laplace transformation is then defined by

$$
\mathrm{FL}_{X}(\mathcal{M}):=p_{2+}\left(p_{1}^{+} \mathcal{M} \stackrel{L}{\otimes} \mathcal{L}\right)[-n] .
$$

Definition 3.5. Let $A=\left(a_{k i}\right)$ be a $d \times n$ integer matrix. Let $\beta \in \mathbb{C}^{d}$. Write $\mathbb{L}_{A}$ for the $\mathbb{Z}$-module of relations among the columns of $A$, and write $\mathcal{D}_{W}$ for the sheaf of rings of algebraic differential operators on $W$. Define

$$
\check{\mathcal{M}}_{A}^{\beta}:=\mathcal{D}_{W} /\left(\left(\check{\square}_{\underline{m}}\right)_{\underline{m} \in \mathbb{L}_{A}},\left(\check{E}_{k}+\beta_{k}\right)_{k=1, \ldots, d}\right),
$$




\section{Hypergeometric Hodge modules}

where

$$
\check{\square}_{\underline{m} \in \mathbb{L}_{A}}:=\prod_{m_{i}>0} w_{i}^{m_{i}}-\prod_{m_{i}<0} w_{i}^{-m_{i}} \text { and } \quad \check{E}_{k}:=\sum_{i=1}^{n} a_{k i} \partial_{w_{i}} w_{i} \quad \text { for } k=1, \ldots, d,
$$

Again, we will often work with the $D_{W}$-module of global sections

$$
\check{M}_{A}^{\beta}:=\Gamma\left(W, \check{\mathcal{M}}_{A}^{\beta}\right)
$$

of the $\mathcal{D}_{W}$-module $\check{\mathcal{M}}_{A}^{\beta}$. Sometimes, we will be interested in the case $\beta=0$ and will write

$$
\check{\mathcal{M}}_{A}:=\check{\mathcal{M}}_{A}^{0} \text { and } M_{A}:=\Gamma\left(W, \check{\mathcal{M}}_{A}\right) \text {. }
$$

Remark 3.6. Notice that $\check{\mathcal{M}}_{A}^{\beta}$ is just a Fourier-Laplace transformation (in all variables) of the GKZ system $\mathcal{M}_{A}^{\beta}$ (cf. Definition 3.1).

The semigroup ring associated with the matrix $A$ is

$$
\mathbb{C}[\mathbb{N} A] \simeq \mathbb{C}[\underline{w}] /\left(\left(\check{\square}_{\underline{m}}\right)_{\underline{m} \in \mathbb{L}_{A}}\right),
$$

where $\mathbb{C}[\underline{w}]$ is the commutative ring $\mathbb{C}\left[w_{1}, \ldots, w_{n}\right]$ and the isomorphism follows from [MS05, Theorem 7.3]. The rings $\mathbb{C}[\underline{w}]$ and $\mathbb{C}[\mathbb{N} A]$ are naturally $\mathbb{Z}^{d}$-graded if we define $\operatorname{deg}\left(w_{j}\right)=\underline{a}_{j}$ for $j=1, \ldots, n$. This is compatible with the $\mathbb{Z}^{d}$-grading of the Weyl algebra $D_{W}$ given by $\operatorname{deg}\left(\partial_{w_{j}}\right)=-\underline{a}_{j}$ and $\operatorname{deg}\left(w_{j}\right)=\underline{a}_{j}$.

Definition 3.7 ([MMW05, Definition 5.2]). Let $N$ be a finitely generated $\mathbb{Z}^{d}$-graded $\mathbb{C}[\underline{w}]$ module. An element $\alpha \in \mathbb{Z}^{d}$ is called a true degree of $N$ if $N_{\alpha}$ is non-zero. A vector $\alpha \in \mathbb{C}^{d}$ is called a quasi-degree of $N$, written $\alpha \in \operatorname{qdeg}(N)$, if $\alpha$ lies in the complex Zariski closure qdeg $(N)$ of the true degrees of $N$ via the natural embedding $\mathbb{Z}^{d} \hookrightarrow \mathbb{C}^{d}$.

Schulze and Walther now define the following set of parameters.

Definition 3.8 ([SW09]). The set

$$
\operatorname{sRes}(A):=\bigcup_{j=1}^{n} \operatorname{sRes}_{j}(A)
$$

where $\operatorname{sRes}_{j}(A):=\left\{\beta \in \mathbb{C}^{d} \mid \beta \in-(\mathbb{N}+1) \underline{a}_{j}+\operatorname{qdeg}\left(\mathbb{C}[\mathbb{N} A] /\left(w_{j}\right)\right)\right\}$, is called the set of strongly resonant parameters of $A$.

The matrix $A$ is called pointed if 0 is the only unit in $\mathbb{N} A$. The matrix $A$ gives rise to a map from a torus $T=\left(\mathbb{C}^{*}\right)^{d}$ with coordinates $\left(t_{1}, \ldots, t_{d}\right)$ into the affine space $W=\mathbb{C}^{n}$ with coordinates $w_{1}, \ldots, w_{n}$ :

$$
h_{A}: T \longrightarrow W, \quad\left(t_{1}, \ldots, t_{d}\right) \longmapsto\left(\underline{t}^{\underline{a}_{1}}, \ldots, \underline{t}^{\underline{a}}\right),
$$

where $\underline{\underline{a}}^{\underline{a}}:=\prod_{k=1}^{d} t_{k}^{a_{k i}}$. Notice that the map $h_{A}$ is affine and is a locally closed embedding; hence, the direct image functor for $\mathcal{D}_{T}$-modules $\left(h_{A}\right)_{+}$is exact.

For a pointed matrix $A$, Schulze and Walther computed the direct image of the twisted structure sheaf

$$
\mathcal{O}_{T}^{\beta}:=\mathcal{D}_{T} / \mathcal{D}_{T} \cdot\left(\partial_{t_{1}} t_{1}+\beta_{1}, \ldots, \partial_{t_{d}} t_{d}+\beta_{d}\right)
$$

under the morphism $h_{A}$. 


\section{T. Reichelt And C. Sevenheck}

Theorem 3.9 ([SW09, Theorem 3.6, Corollary 3.7]). Let $A$ a pointed $d \times n$ integer matrix satisfying $\mathbb{Z} A=\mathbb{Z}^{d}$; then the following statements are equivalent:

(i) $\beta \notin \operatorname{sRes}(A)$,

(ii) $\check{\mathcal{M}}_{A}^{\beta} \simeq\left(h_{A}\right)_{+} \mathcal{O}_{T}^{\beta}$,

(iii) left multiplication with $w_{i}$ is invertible on $\check{M}_{A}^{\beta}$ for $i=1, \ldots, n$.

Notice that Schulze and Walther [SW09] use the GKZ system $\mathcal{M}_{A}^{\beta}$ and the convention $\operatorname{deg}\left(\partial_{\lambda_{j}}\right)=\underline{a}_{j}$. We will use $\check{\mathcal{M}}_{A}^{\beta}$ and $\operatorname{deg}\left(w_{j}\right)=\underline{a}_{j}$ instead.

The aim of section is to generalize the implication (i) $\Rightarrow$ (ii) to the case of a non-pointed matrix $A$. For this, we set $\underline{a}_{0}:=0$. We will associate with the matrix $A$ the homogenized $(d+1) \times(n+1)$ matrix $\widetilde{A}$ with columns $\underline{\widetilde{a}}_{i}:=\left(1, \underline{a}_{i}\right)$ for $i=0, \ldots, n$. Notice that $\mathbb{Z} \widetilde{A}=\mathbb{Z}^{d+1}$ holds and that the matrix $\widetilde{A}$ is pointed in any case. Now, consider the augmented map

$$
h_{\widetilde{A}}: \widetilde{T} \longrightarrow \widetilde{W}, \quad\left(t_{0}, \ldots, t_{d}\right) \longmapsto\left(t_{0} \underline{t}^{\underline{a}_{0}}, t_{0} \underline{\underline{t}}^{\underline{a_{1}}}, \ldots, t_{0} \underline{\underline{t}}^{\underline{a_{n}}}\right),
$$

where $\widetilde{T}=\left(\mathbb{C}^{*}\right)^{d+1}$ and $\widetilde{W}=\mathbb{C}^{n+1}$ with coordinates $w_{0}, \ldots, w_{n}$. Let $\widetilde{W}_{0}$ be the subvariety of $\widetilde{W}$ given by $w_{0} \neq 0$, and denote by $k_{0}: \widetilde{W}_{0} \rightarrow \widetilde{W}$ the canonical embedding. The map $h_{\widetilde{A}}$ factors through $\widetilde{W}_{0}$, which gives rise to a map $h_{0}$ with $h_{\widetilde{A}}=k_{0} \circ h_{0}$. We get the following commutative diagram:

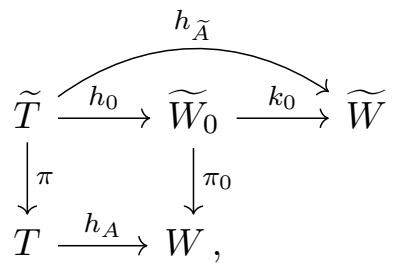

where $\pi$ is the projection which forgets the first coordinate and $\pi_{0}$ is given by

$$
\pi_{0}: \widetilde{W}_{0} \longrightarrow W, \quad\left(w_{0}, w_{1}, \ldots, w_{n}\right) \longmapsto\left(w_{1} / w_{0}, \ldots, w_{n} / w_{0}\right) .
$$

Lemma 3.10. For each $\beta_{0} \in \mathbb{Z}$, we have an isomorphism

$$
\mathcal{H}^{0}\left(h_{A+} \mathcal{O}_{T}^{\beta}\right) \simeq \mathcal{H}^{0}\left(\pi_{0+} k_{0}^{+}\left(h_{\widetilde{A}+} \mathcal{O}_{\widetilde{T}}^{\left(\beta_{0}, \beta\right)}\right)\right) .
$$

Proof. We show the claim by using the isomorphisms

$$
\begin{aligned}
\mathcal{H}^{0} h_{A+} \mathcal{O}_{T}^{\beta} & \simeq \mathcal{H}^{0} h_{A+} \mathcal{H}^{0} \pi_{+} \mathcal{O}_{\widetilde{T}}^{\left(\beta_{0}, \beta\right)} \simeq \mathcal{H}^{0} h_{A+} \pi_{+} \mathcal{O}_{\widetilde{T}}^{\left(\beta_{0}, \beta\right)} \simeq \mathcal{H}^{0} \pi_{0+} h_{0+} \mathcal{O}_{\widetilde{T}}^{\left(\beta_{0}, \beta\right)} \\
& \simeq \mathcal{H}^{0} \pi_{0+} k_{0}^{+} k_{0+} h_{0+} \mathcal{O}_{\widetilde{T}}^{\left(\beta_{0}, \beta\right)} \simeq \mathcal{H}^{0} \pi_{0+} k_{0}^{+} h_{\widetilde{A}+} \mathcal{O}_{\widetilde{T}}^{\left(\beta_{0}, \beta\right)} .
\end{aligned}
$$

The first isomorphism follows from the fact that $\pi$ is a projection with fiber $\mathbb{C}^{*}$; the second isomorphism follows from the exactness of $\left(h_{A}\right)_{+}$and the fourth from the fact that $k_{0}^{+}\left(k_{0}\right)_{+} \simeq$ $\operatorname{id}_{\widetilde{W}_{0}}$.

The following proposition is the generalization of Theorem 3.9 to the non-pointed case.

Proposition 3.11. Let $A=\left(a_{k i}\right)$ be a $d \times n$ integer matrix satisfying $\mathbb{Z} A=\mathbb{Z}^{d}$, and let $\beta \in \mathbb{C}^{d}$ with $\beta \notin \operatorname{sRes}(A)$; then $\mathcal{H}^{0}\left(\left(h_{A}\right)_{+} \mathcal{O}_{T}^{\beta}\right)$ is isomorphic to $\check{\mathcal{M}}_{A}^{\beta}$.

Proof. The proof relies on Lemma 3.10 and the theorem of Schulze and Walther in the pointed case. Notice that we can find a $\beta_{0} \in \mathbb{Z}$ with $\beta_{0} \gg 0$ such that $\left(\beta_{0}, \beta\right) \notin \operatorname{sRes}(\widetilde{A})$ by $[\operatorname{Rei14}$, 


\section{HypergeOMetric Hodge MOdules}

Lemma 1.16] (where the statement is formulated for $\beta \in \mathbb{Q}^{d}$, but the proof carries over almost word for word in this more general case).

Consider the following isomorphism on $\widetilde{W}_{0}$ :

$$
f: \widetilde{W}_{0} \longrightarrow \mathbb{C}_{w_{0}}^{*} \times W, \quad\left(w_{0}, \ldots, w_{n}\right) \longmapsto\left(\left(w_{0}, w_{1} / w_{0}, \ldots, w_{n} / w_{0}\right)\right.
$$

together with the canonical projection $p: W \times \mathbb{C}_{w_{0}}^{*} \rightarrow W$ which forgets the first coordinate. Then $\pi_{0}$ factors as $\pi_{0}=p \circ f$, which gives (using Lemma 3.10)

$$
\begin{aligned}
\mathcal{H}^{0}\left(\left(h_{A}\right)_{+} \mathcal{O}_{T}^{\beta}\right) & \simeq \mathcal{H}^{0}\left(\left(\pi_{0}\right)_{+}\left(\left(h_{\widetilde{A}}\right)_{+} \mathcal{O}_{\widetilde{T}}^{\left(\beta_{0}, \beta\right)}\right)_{\mid \widetilde{W}_{0}}\right) \simeq \mathcal{H}^{0}\left(p_{+} f_{+}\left(\left(h_{\widetilde{A}}\right)_{+} \mathcal{O}_{\widetilde{T}}^{\left(\beta_{0}, \beta\right)}\right)_{\mid \widetilde{W}_{0}}\right) \\
& \simeq \mathcal{H}^{0}\left(p_{+} f_{+}\left(\check{\mathcal{M}}_{\widetilde{A}}^{\left(\beta_{0}, \beta\right)}\right)_{\mid \widetilde{W}_{0}}\right) .
\end{aligned}
$$

The $\mathcal{D}$-module $\mathcal{H}^{0} f_{+}\left(\check{\mathcal{M}}_{\widetilde{A}}^{\left(\beta_{0}, \beta\right)}\right)_{\mid \widetilde{W}_{0}}$ is isomorphic to $\mathcal{D}_{W \times \mathbb{C}_{w_{0}}^{*}} / \mathcal{I}_{0}^{\prime}$, where $\mathcal{I}_{0}^{\prime}$ is generated by

$$
\check{\square}_{\underline{m} \in \mathbb{L}_{A}}=\prod_{i: m_{i}>0} w_{i}^{m_{i}}-\prod_{i: m_{i}<0} w_{i}^{-m_{i}}
$$

and

$$
Z_{0}=\partial_{w_{0}} w_{0}+\beta_{0} \quad \text { and } \quad E_{k}=\sum_{i=1}^{n} a_{k i} \partial_{w_{i}} w_{i}+\beta_{k} .
$$

Hence, $\mathcal{H}^{0} f_{+}\left(\check{\mathcal{M}}_{\widetilde{A}}^{\left(\beta_{0}, \beta\right)}\right)_{\mid \widetilde{W}_{0}}$ is isomorphic to $\check{\mathcal{M}}_{A}^{\beta} \otimes \mathcal{D}_{\mathbb{C}_{w_{0}}^{*}} /\left(\partial_{w_{0}} w_{0}+\beta_{0}\right)$ as a $\mathcal{D}$-module. We therefore have

$$
\begin{aligned}
\mathcal{H}^{0}\left(p_{+} f_{+}\left(\check{\mathcal{M}}_{\widetilde{A}}^{\left(\beta_{0}, \beta\right)}\right)_{\mid \widetilde{W}_{0}}\right) & \simeq \mathcal{H}^{0}\left(p_{+} \mathcal{H}^{0} f_{+}\left(\check{\mathcal{M}}_{\widetilde{A}}^{\left(\beta_{0}, \beta\right)}\right)_{\mid \widetilde{W}_{0}}\right) \\
& \simeq \mathcal{H}^{0} p_{+}\left(\check{\mathcal{M}}_{A}^{\beta} \otimes \mathcal{D}_{\mathbb{C}_{w_{0}}^{*}} /\left(\partial_{w_{0}} w_{0}+\beta_{0}\right)\right) \simeq \check{\mathcal{M}}_{A}^{\beta} .
\end{aligned}
$$

\section{Hodge filtration on torus embeddings}

The aim of this section is to compute explicitly the Hodge filtration of $\left(h_{A}\right)_{+} \mathcal{O}_{T}^{\beta}$ as a mixed Hodge module for certain values of $\beta$ (cf. Theorem 4.17). We will use this result in Section 5 , where the behavior of mixed Hodge modules obtained by such torus embeddings under the twisted Radon transformation is studied.

\subsection{V-filtration}

As above, let $A$ be a $d \times n$ integer matrix such that $\mathbb{Z} A=\mathbb{Z}^{d}$. In this subsection, we additionally assume that the matrix $A$ satisfies the conditions

$$
\mathbb{N} A=\mathbb{Z}^{d} \cap \mathbb{R}_{\geqslant 0} A \text { and } \quad \mathbb{N} A \neq \mathbb{Z}^{d},
$$

where $\mathbb{R}_{\geqslant 0} A$ is the cone generated by the columns of $A$. As already noticed above, the first condition is equivalent to the fact that the semigroup ring $\mathbb{C}[\mathbb{N} A]$ is normal (see, for example, [BH98, Section 6.1]). We will again consider the locally closed embedding

$$
h_{A}: T \longrightarrow W, \quad\left(t_{1}, \ldots, t_{d}\right) \longmapsto\left(\underline{t}^{\underline{a}_{1}}, \ldots, \underline{t}^{\underline{a}}\right) .
$$

Put $D:=\left\{w_{1} \cdot \ldots \cdot w_{n}=0\right\} \subset W$ and $W^{*}:=W \backslash D$, and consider the decomposition $h_{A}=l_{A} \circ k_{A}$, where

$$
k_{A}: T \longrightarrow W^{*}, \quad\left(t_{1}, \ldots, t_{d}\right) \longmapsto\left(\underline{t}^{\underline{a}_{1}}, \ldots, \underline{t}^{\underline{a}}\right),
$$

and where $l_{A}: W^{*} \rightarrow W$ is the canonical open embedding. 


\section{T. Reichelt And C. Sevenheck}

Lemma 4.1. The morphism $k_{A}: T \rightarrow W^{*}$ is a closed embedding.

Proof. This is clear, as the image of $k_{A}$ is precisely the vanishing locus of the ideal $\left(\check{\square}_{\underline{m}}\right)_{\underline{m} \in \mathbb{L}_{A}} \subset$ $\Gamma\left(W^{*}, \mathcal{O}_{W^{*}}\right)$.

The aim of this subsection is to compute parts of the canonical (descending) $V$-filtration (or Kashiwara-Malgrange filtration) of $\check{\mathcal{M}}_{A}^{\beta} \simeq h_{A+} \mathcal{O}_{T}^{\beta}$ along the normal crossing divisor $D$ for certain values of $\beta$.

We review very briefly some facts about the $V$-filtration for differential modules. Let $X=$ Spec $(R)$ be a smooth affine variety and $Y=\operatorname{div}(t)$ be a smooth reduced principal divisor. Denote the corresponding ideal by $I=(t)$. As before, let $D_{X}=\Gamma\left(X, \mathcal{D}_{X}\right)$ be the ring of algebraic differential operators on $X$; then the $V$-filtration on $D_{X}$ is defined by

$$
V^{k} D_{X}=\left\{P \in D_{X} \mid P I^{j} \subset I^{j+k} \text { for any } j \in \mathbb{Z}\right\},
$$

where $I^{j}=R$ for $j \leqslant 0$. One has

$$
V^{k} D_{X}=t^{k} V^{0} D_{X} \quad \text { and } \quad V^{-k} D_{X}=\sum_{0 \leqslant j \leqslant k} \partial_{t}^{j} V^{0} D_{X}
$$

Choose a total ordering $<$ on $\mathbb{C}$ such that for any $\alpha, \beta \in \mathbb{C}$, the following conditions hold:

(1) $\alpha<\alpha+1$,

(2) $\alpha<\beta$ if and only if $\alpha+1<\beta+1$,

(3) $\alpha<\beta+m$ for some $m \in \mathbb{Z}$.

We recall the definition of the canonical $V$-filtration (see, for example, [Sai93, Section 1]).

Definition 4.2 . Let $N$ be a coherent $D_{X}$-module. The canonical $V$-filtration (or KashiwaraMalgrange filtration) is an exhaustive filtration on $N$ indexed discretely by $\mathbb{C}$ with total order as above; it is uniquely determined by the following conditions:

(i) $\left(V^{k} D_{X}\right)\left(V^{\alpha} N\right) \subset V^{\alpha+k} N$ for all $k, \alpha$.

(ii) The module $V^{\alpha} N$ is coherent over $V^{0} D_{X}$ for any $\alpha$.

(iii) $t\left(V^{\alpha} N\right)=V^{\alpha+1} N$ for $\alpha \gg 0$,

(iv) The action of $\partial_{t} t-\alpha$ on $\operatorname{Gr}_{V}^{\alpha} N=V^{\alpha} N / V^{>\alpha} N$ is nilpotent, where $V^{>\alpha} N:=\bigcup_{\beta>\alpha} V^{\beta}$.

The canonical $V$-filtration is unique if it exists. Its existence is guaranteed if $N$ is $D_{X^{-}}$ holonomic.

We reduce the computation of the $V$-filtration on $\check{M}_{A}^{\beta}$ along the possibly singular divisor $D$ to the computation of a $V$-filtration along a smooth divisor by considering the following graph embedding:

$$
i_{g}: W \longrightarrow W \times \mathbb{C}_{t}, \quad\left(w_{1}, \ldots, w_{n}\right) \longmapsto\left(w_{1}, \ldots, w_{n}, w_{1} \cdot \ldots \cdot w_{n}\right) .
$$

Instead of computing the $V$-filtration on $\check{M}_{A}^{\beta}$, we will compute it on $\Gamma\left(W \times \mathbb{C}_{t}, \mathcal{H}^{0}\left(i_{g+} \check{\mathcal{M}}_{A}^{\beta}\right)\right)$ along $t=0$ (notice that $i_{g}$ is an affine embedding; hence, $i_{g+}$ is exact). In order to compute the direct image, we consider the composed map

$$
i_{g} \circ h_{A}: T \longrightarrow W \times \mathbb{C}_{t}, \quad\left(t_{1}, \ldots, t_{d}\right) \longmapsto\left(\underline{t}^{\underline{a}}, \ldots, \underline{t}^{\underline{a}}, \underline{t}^{\underline{a_{1}}+\cdots+\underline{a}_{n}}\right) .
$$

Notice that the matrix $A^{\prime}$, which is built from the columns $\underline{a}_{1}, \ldots, \underline{a}_{n}, \underline{a}_{1}+\cdots+\underline{a}_{n}$, gives a saturated semigroup $\mathbb{N} A^{\prime}=\mathbb{N} A$. Hence, we can apply Proposition 3.11 again to compute

$$
\check{\mathcal{M}}_{A^{\prime}}^{\beta} \simeq \mathcal{H}^{0} i_{g+} \check{\mathcal{M}}_{A}^{\beta} \simeq \mathcal{H}^{0}\left(i_{g} \circ h_{A}\right)_{+} \mathcal{O}_{T}^{\beta} .
$$




\section{Hypergeometric Hodge modules}

This means that $\mathcal{H}^{0} i_{g+} \check{\mathcal{M}}_{A}^{\beta}$ is a cyclic $\mathcal{D}_{W \times \mathbb{C}_{t}}$-module $\mathcal{D}_{W \times \mathbb{C}_{t}} / \mathcal{I}^{\prime}$ for $\mathcal{I}^{\prime}$ generated by

$$
\check{E}_{k}^{\prime}:=\sum_{i=1}^{n} a_{k i} \partial_{w_{i}} w_{i}+c_{k} \partial_{t} t+\beta_{k} \quad \text { for } k=1, \ldots, d,
$$

where $c_{k}=a_{k 1}+\cdots+a_{k n}$ is the $k$ th component of $c \in \mathbb{Z}^{d}$ and

$$
\check{\square}_{\underline{m} \in \mathbb{L}_{A^{\prime}}}:= \begin{cases}\prod_{m_{i}>0} w_{i}^{m_{i}} t^{m_{n+1}}-\prod_{m_{i}<0} w_{i}^{-m_{i}} & \text { for } m_{n+1} \geqslant 0, \\ \prod_{m_{i}>0} w_{i}^{m_{i}}-\prod_{m_{i}<0} w_{i}^{-m_{i}} t^{-m_{n+1}} & \text { for } m_{n+1}<0\end{cases}
$$

with $\mathbb{L}_{A^{\prime}}$ the $\mathbb{Z}$-module of relations among the columns of $A^{\prime}$.

We are going to use the following characterization of the canonical $V$-filtration along $t=0$.

Proposition 4.3 ([MM04, Definition 4.3-3, Proposition 4.3-9]). Let $n \in N$, and set $E:=\partial_{t} t$. The Bernstein-Sato polynomial of $n$ is the unitary polynomial of smallest degree satisfying

$$
b(E) n \in V^{1}\left(D_{X}\right) n .
$$

We denote it by $b_{n}(x) \in \mathbb{C}[x]$ and denote the set of roots of $b_{n}(x)$ by $\operatorname{ord}(n)$. The canonical $V$-filtration on $N$ is then given by

$$
V^{\alpha} N=\{n \in N \mid \operatorname{ord}(n) \subset[\alpha, \infty)\} .
$$

We will use this characterization to compute the canonical $V$-filtration on $\check{M}_{A^{\prime}}^{\beta}$ along $t=0$ for certain $\beta \in \mathbb{R}^{d}$.

Let $\underline{c}:=\underline{a}_{1}+\cdots+\underline{a}_{n}$. For all facets $F$ of $\mathbb{R}_{\geqslant 0} A^{\prime}=\mathbb{R}_{\geqslant 0} A$, let $0 \neq \underline{n}_{F} \in \mathbb{Z}^{d}$ be the uniquely determined primitive, inward-pointing, normal vector of $F$; that is, $\underline{n}_{F}$ satisfies $\left\langle\underline{n}_{F}, F\right\rangle=0$, $\left\langle\underline{n}_{F}, \mathbb{N} A\right\rangle \subset \mathbb{Z}_{\geqslant 0}$ and $\lambda \cdot \underline{n}_{F} \notin \mathbb{Z}^{d}$ for $\lambda \in[0,1)$ (where $\langle\cdot, \cdot\rangle$ is the Euclidean pairing). Set

$$
e_{F}:=\left\langle\underline{n}_{F}, \underline{c}\right\rangle \in \mathbb{Z}_{\geqslant 0} .
$$

We show that $e_{F}$ is always positive. We have $\underline{c} \neq 0$ since otherwise $0=-\underline{a}_{1}-\cdots-\underline{a}_{n} \in \mathbb{N} A$, and therefore $-\underline{a}_{i} \in \mathbb{N} A$ for all $i \in\{1, \ldots, n\}$, which contradicts the assumption $\mathbb{N} A \neq \mathbb{Z}^{d}$. Furthermore, $\underline{c}$ lies in the interior of $\mathbb{R}_{\geqslant 0} A^{\prime}$. In order to see this, assume to the contrary that $\underline{c}$ lies on some facet $F$ of $\mathbb{R}_{\geqslant 0} A^{\prime}$. Then $\left\langle\underline{n}_{F}, \underline{c}\right\rangle=0$ holds. For $\underline{a}_{i} \notin F$, we have on the one hand $\underline{c}-\underline{a}_{i} \in \mathbb{N} A$ and on the other hand $\left\langle\underline{c}-\underline{a}_{i}, \underline{n}_{F}\right\rangle<0$, which gives a contradiction. Hence, $\underline{c}$ is in the interior of $\mathbb{R}_{\geqslant 0} A$, which shows $e_{F} \in \mathbb{Z}_{>0}$.

We define the following set of admissible parameters $\beta$ :

$$
\mathfrak{A}_{A}:=\bigcap_{F: \text { facet }}\left\{\mathbb{R} \cdot F-\left[0, \frac{1}{e_{F}}\right) \cdot \underline{c}\right\} .
$$

Lemma 4.4. As above, suppose $\mathbb{N} A=\mathbb{Z}^{d} \cap \mathbb{R}_{\geqslant 0} A$. Consider the cyclic $D_{W \times \mathbb{C}_{t}-\text { module } \check{M}_{A^{\prime}}^{\beta} \text { and }}$ its generator $[1] \in \check{M}_{A^{\prime}}$. Then we have ord([1]) $\subset[0,1)$ if $\beta \in \mathfrak{A}_{A}$.

Proof. It was shown in [RSW18, Theorem 3.5] that the roots of $b_{[1]}(x)$ for $[1] \in \check{M}_{A}^{\beta}$ are contained in the set $\left\{\epsilon \in \mathbb{C} \mid \epsilon \cdot \underline{c} \in \operatorname{qdeg}\left(\mathbb{C}\left[\mathbb{N} A^{\prime}\right] /(t)\right)-\beta\right\}$, which is discrete since $\underline{c}$ lies in the interior of $\mathbb{R}_{\geqslant 0} A^{\prime}=\mathbb{R}_{\geqslant 0} A$ and qdeg $\left(\mathbb{C}\left[\mathbb{N} A^{\prime}\right] /(t)\right)$ is a finite union of parallel translates of the complex span of faces of $\mathbb{R}_{\geqslant 0} A^{\prime}$ (cf. [MMW05]). We will now compute an estimate of the quasi-degrees $\operatorname{qdeg}\left(\mathbb{C}\left[\mathbb{N} A^{\prime}\right] /(t)\right)$. For this, we remark that $0=[P] \in \mathbb{C}\left[\mathbb{N} A^{\prime}\right] /(t)$ for $P \in \mathbb{C}\left[\mathbb{N} A^{\prime}\right]$ if and only if there exists a $P^{\prime} \in \mathbb{C}\left[\mathbb{N} A^{\prime}\right]$ with $P=P^{\prime} \cdot t$. In this case, we have $\operatorname{deg}(P) \in \mathbb{N} A+\underline{c}$. 


\section{T. Reichelt And C. Sevenheck}

Set $L_{F}:=\left\{\left(k / e_{F}\right) \cdot \underline{c}+\mathbb{C} \cdot F \mid k=0, \ldots, e_{F}-1\right\}$. Then $L=\bigcup_{F \text { : facet }} L_{F}$ is Zariski closed, and we will show that the set $\operatorname{deg}\left(\mathbb{C}\left[\mathbb{N} A^{\prime}\right] /(t)\right)$ is contained in L. Let $P \in \mathbb{C}\left[\mathbb{N} A^{\prime}\right]$ with $0 \neq$ $[P] \in \mathbb{C}\left[\mathbb{N} A^{\prime}\right] /(t)$, and set $\underline{p}:=\operatorname{deg}(P) \in \mathbb{N} A$. Since $-\underline{c} \notin \mathbb{R}_{\geqslant 0} A$, there exist a facet $F$ and some $\lambda \in[0,1)$ such that $\underline{p}-\lambda \underline{c} \in F$; that is, $\underline{p}=\lambda \underline{c}+\underline{f}$ for some $\underline{f} \in F$. We have $\lambda \cdot e_{F}=\left\langle\lambda \underline{c}+\underline{f}, \underline{n}_{F}\right\rangle=$ $\left\langle\underline{p}, \underline{n}_{F}\right\rangle \in \mathbb{Z}_{\geqslant 0}$. Hence, $\underline{p} \in L_{F} \subset L$.

Since qdeg $\left(\mathbb{C}\left[\mathbb{N} A^{\prime}\right] /(t)\right)$ is by definition the Zariski closure of $\operatorname{deg}\left(\mathbb{C}\left[\mathbb{N} A^{\prime}\right] /(t)\right)$, the former set is contained in $L$. In particular, this shows that the roots of $b_{[1]}(x)$ are contained in the set $\{\epsilon \in \mathbb{C} \mid \epsilon \cdot \underline{c} \in L-\beta\}$. Since $L$ is a union of hypersurfaces which are defined over $\mathbb{R}$ and $\underline{c} \in \mathbb{Z}^{d}$ and $\beta \in \mathbb{R}^{d}$, this set is equal to $\{\epsilon \in \mathbb{R} \mid \epsilon \cdot \underline{c} \in L-\beta\}$. Hence, for $\beta \in \bigcap_{F \text { : facet }}\left\{\mathbb{R} \cdot F-\left[0,1 / e_{F}\right) \cdot \underline{c}\right\}$, we can guarantee that the roots of $b_{[1]}(x)$ are contained in $[0,1)$.

We will prove a basic lemma on the set $\mathfrak{A}_{A}$ which will be of importance later.

Lemma 4.5. Suppose $\mathbb{N} A=\mathbb{Z}^{d} \cap \mathbb{R}_{\geqslant 0}$. Then $\mathfrak{A}_{A} \cap \operatorname{sRes}(A)=\emptyset$.

Proof. Recall that $\operatorname{sRes}(A)=\bigcup_{j=1}^{n} \operatorname{sRes}_{j}(A)=\bigcup_{j=1}^{n}-(\mathbb{N}+1) \underline{a}_{j}+\operatorname{qdeg}\left(\mathbb{C}[\mathbb{N} A] /\left(\left(w_{j}\right)\right)\right.$. Therefore, it is enough to show that

$$
\mathfrak{A}_{A} \cap\left\{-(\mathbb{N}+1) \underline{a}_{j}+\operatorname{qdeg}\left(\mathbb{C}[\mathbb{N} A] /\left(w_{j}\right)\right)\right\}=\emptyset
$$

holds. The following estimate of the quasi-degrees of $\left.\mathbb{C}[\mathbb{N} A] /\left(w_{j}\right)\right)$ can be shown similarly as in the proof of the lemma above:

$$
\operatorname{qdeg}\left(\mathbb{C}[\mathbb{N} A] /\left(\left(w_{j}\right)\right) \subset L_{j}:=\bigcup_{F: \underline{a}_{j} \notin F}\left\{\frac{k}{e_{F, j}} \cdot \underline{a}_{j}+\mathbb{C} \cdot F \mid k=0, \ldots, e_{F, j}-1\right\},\right.
$$

where $e_{F, j}:=\left\langle\underline{n}_{F}, \underline{a}_{j}\right\rangle$. Hence, it is enough to show that for each $j \in\{1, \ldots, n\}$ and each facet $F$ with $\underline{a}_{j} \notin F$, the following holds:

$$
\left\{\mathbb{R} \cdot F-\left[0, \frac{1}{e_{F}}\right) \cdot \underline{c}\right\} \cap\left\{-(\mathbb{N}+1) \underline{a}_{j}+\bigcup_{k=0}^{e_{F, j}-1} \frac{k}{e_{F, j}} \cdot \underline{a}_{j}+\mathbb{R} \cdot F\right\}=\emptyset .
$$

Since $F$ has codimension one in $\mathbb{R}^{d}$ and $\underline{a}_{j}, \underline{c} \notin \mathbb{R} \cdot F$, we can write $\underline{c}=\lambda \underline{a}_{j}+f$ for some $f \in \mathbb{R} \cdot F$. We get $e_{F}=\lambda e_{F, j}$. We conclude that (4.4) is equivalent to

$$
\left\{\mathbb{R} \cdot F-\left[0, \frac{1}{e_{F, j}}\right) \cdot \underline{a}_{j}\right\} \cap\left\{-(\mathbb{N}+1) \underline{a}_{j}+\bigcup_{k=0}^{e_{F, j}-1} \frac{k}{e_{F, j}} \cdot \underline{a}_{j}+\mathbb{R} \cdot F\right\}=\emptyset .
$$

But this holds since $\left(-1 / e_{F, j}, 0\right] \cap\left\{-(\mathbb{N}+1)+\left\{0,1 / e_{F, j}, \ldots,\left(e_{F, j}-1\right) / e_{F, j}\right\}\right\}=\emptyset$.

Example 4.6. The sets $\operatorname{sRes}(A)$ and $\mathfrak{A}_{A}$ for the matrix

$$
A=\left(\begin{array}{rrrr}
-1 & 0 & 1 & 2 \\
1 & 1 & 1 & 1
\end{array}\right)
$$

are sketched in Figure 1.

We give another estimate of the set $\mathfrak{A}_{A}$.

Lemma 4.7. Suppose $\mathbb{N} A=\mathbb{Z}^{d} \cap \mathbb{R}_{\geqslant 0} A$. Let $\underline{b} \in \mathbb{Z}^{d} \cap \operatorname{int}\left(\mathbb{R}_{\geqslant 0} A\right)$; then $\mathfrak{A}_{A} \subset-\underline{b}+\operatorname{int}\left(\mathbb{R}_{\geqslant 0} A\right)$. 


\section{Hypergeometric Hodge modules}

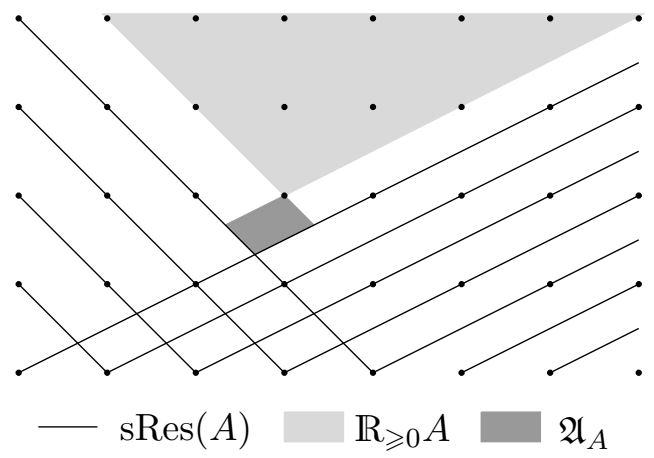

Figure 1. The sets $\operatorname{sRes}(A)$ and $\mathfrak{A}_{A}$

Proof. Let $F$ be a face of $\mathbb{R}_{\geqslant 0} A$. Since $F$ has codimension and $\underline{b}, \underline{c} \notin \mathbb{R} F$, we can write $\underline{c}=\lambda \underline{b}+f$ for some $f \in \mathbb{R} \cdot F$. If we set $e_{F, \underline{b}}:=\left\langle\underline{n}_{F}, \underline{b}\right\rangle \in \mathbb{Z}_{>0}$, we get $e_{F}=\lambda e_{F, \underline{b}}$. Hence,

$$
\begin{aligned}
\left.\left\{\mathbb{R} \cdot F-\left[0, \frac{1}{e_{F}}\right) \cdot \underline{c}\right)\right\} & =\left\{\mathbb{R} \cdot F-\left[0, \frac{1}{e_{F, \underline{b}}}\right) \cdot \underline{b}\right\} \subset\{\mathbb{R} \cdot F-[0,1) \underline{b}\} \\
& \subset\{\mathbb{R} \cdot F-\underline{b}+(0, \infty) \underline{b}\} \subset-\underline{b}+\left\{\mathbb{R} \cdot F+(0, \infty) n_{\underline{F}}\right\},
\end{aligned}
$$

and therefore

$$
\left.\mathfrak{A}=\bigcap_{F}\left\{\mathbb{R} \cdot F-\left[0, \frac{1}{e_{F}}\right) \cdot \underline{c}\right)\right\} \subset-\underline{b}+\operatorname{int}\left(\mathbb{R}_{\geqslant 0} A\right) .
$$

Next, we draw a consequence for the canonical $V$-filtration with respect to $t=0$ on $\mathcal{H}^{0} i_{g+} \check{\mathcal{M}}_{A}^{\beta}$. We will not compute all of its filtration steps but only those corresponding to integer indices, which is sufficient for our purpose. For this, consider the induced $V$-filtration on $\check{M}_{A^{\prime}}^{\beta}=\Gamma\left(W \times \mathbb{C}_{t}\right.$, $\left.\mathcal{H}^{0} i_{g+} \check{\mathcal{M}}_{A}^{\beta}\right)$

$$
V_{\text {ind }}^{k} \check{M}_{A^{\prime}}^{\beta}:=\left\{[P] \in \check{M}_{A^{\prime}}^{\beta} \mid P \in V^{k} D_{W \times \mathbb{C}_{t}}\right\} .
$$

It is readily checked that $V_{\text {ind }}^{k} \check{M}_{A^{\prime}}^{\beta}$ is a good $V$-filtration on $\check{M}_{A^{\prime}}^{\beta}$. As $\check{M}_{A^{\prime}}$ is holonomic, hence specializable along any smooth hypersurface, it admits a Bernstein polynomial $b_{V_{\text {ind }}}(x)$ in the sense of [MM04, Définition 4.2-3]. On the other hand, for any section $\sigma: \mathbb{C} / \mathbb{Z} \rightarrow \mathbb{C}$ of the canonical projection $\mathbb{C} \rightarrow \mathbb{C} / \mathbb{Z}$, there is a unique good ( $\mathbb{Z}$-indexed) $V$-filtration $V_{\sigma}^{\bullet} \check{M}_{A^{\prime}}^{\beta}$ on $\check{M}_{A^{\prime}}^{\beta}$ such that the roots of $b_{V_{\sigma}}(x)$ lie in $\operatorname{Im}(\sigma)$; see [MM04, Proposition 4.2-6]. From this, we deduce the following result, which describes the integral part of the canonical $V$-filtration on $\check{M}_{A^{\prime}}^{\beta}$.

Proposition 4.8. If $\mathbb{N} A=\mathbb{Z}^{d} \cap \mathbb{R}_{\geqslant 0} A$ and $\beta \in \mathfrak{A}_{A}$, then for any $k \in \mathbb{Z}$, we have the equality $V^{k} \check{M}_{A^{\prime}}^{\beta}=V_{\text {ind }}^{k} \check{M}_{A^{\prime}}^{\beta}$.

Proof. Recall (see [MM04, Proposition 4.3-5]) that we have $V^{\alpha+k} \check{M}_{A^{\prime}}^{\beta}=V_{\sigma_{\alpha}}^{k} \check{M}_{A^{\prime}}^{\beta}$ for any $\alpha \in \mathbb{C}$ and $k \in \mathbb{Z}$, where $\sigma_{\alpha}: \mathbb{C} / \mathbb{Z} \rightarrow \mathbb{C}$ is the section of $\mathbb{C} \rightarrow \mathbb{C} / \mathbb{Z}$ with image equal to $[\alpha, \alpha+1)$. Hence, in order to prove the proposition, it is enough to show that $V_{\sigma_{0}}^{k} \check{M}_{A^{\prime}}^{\beta}=V_{\text {ind }}^{k} \check{M}_{A^{\prime}}^{\beta}$. Using [MM04, Proposition 4.2-6], it remains to show that the roots of the Bernstein polynomial $b_{V_{\text {ind }}}(x)$ are contained in $[0,1)$. 


\section{T. Reichelt And C. Sevenheck}

An element $[P]$ of $V_{\text {ind }}^{k} \check{M}_{A^{\prime}}^{\beta}$ for $k \geqslant 0$ can be written as

$$
[P]=\left[\sum_{i=0}^{l} t^{k}\left(\partial_{t} t\right)^{i} P_{i}\right]+[R],
$$

where $[R] \in V_{\text {ind }}^{k+1} \check{M}_{A^{\prime}}^{\beta}$ and $P_{i} \in \mathbb{C}\left[w_{1}, \ldots, w_{n}\right]\left\langle\partial_{w_{1}}, \ldots, \partial_{w_{n}}\right\rangle$. We have

$$
\begin{aligned}
b_{[1]}\left(\partial_{t} t-k\right) \cdot[P] & =\left[\sum_{i=0}^{l} t^{k}\left(\partial_{t} t\right)^{i} P_{i} \cdot b_{[1]}\left(\partial_{t} t\right)\right]+b_{[1]}\left(\partial_{t} t-k\right) \cdot[R] \\
& =\sum_{i=0}^{l} t^{k}\left(\partial_{t} t\right)^{i} P_{i} \cdot b_{[1]}\left(\partial_{t} t\right) \cdot[1]+b_{[1]}\left(\partial_{t} t-k\right) \cdot[R] .
\end{aligned}
$$

But $\sum_{i=0}^{l} t^{k}\left(\partial_{t} t\right)^{i} P_{i} \cdot b_{[1]}\left(\partial_{t} t\right) \cdot[1] \in V_{\text {ind }}^{k+1} \check{M}_{A^{\prime}}^{\beta}$ because $\sum_{i=0}^{l} t^{k}\left(\partial_{t} t\right)^{i} P_{i} \in V^{k} D$ and $b_{[1]}\left(\partial_{t} t\right) \cdot[1] \in$ $V_{\text {ind }}^{1} \check{M}_{A^{\prime}}^{\beta}$. Therefore,

$$
b_{[1]}\left(\partial_{t} t-k\right) \cdot[P] \in V_{\text {ind }}^{k+1} \check{M}_{A^{\prime}}^{\beta} .
$$

Now, let $[P] \in V_{\text {ind }}^{-k} \check{M}_{A^{\prime}}^{\beta}$ with $k>0$. It can be written as

$$
[P]=\left[\sum_{i=0}^{l} \partial_{t}^{k}\left(\partial_{t} t\right)^{i} P_{i}\right]+[R],
$$

where $[R] \in V_{\text {ind }}^{k+1} \check{M}_{A^{\prime}}^{\beta}$. By a similar argument, we have $b_{[1]}\left(\partial_{t} t+k\right) \cdot[P] \in V_{\text {ind }}^{-k+1} \check{M}_{A^{\prime}}^{\beta}$. This shows $b_{V_{\text {ind }}}(x) \mid b_{[1]}(x)$. Because of Lemma 4.4 , the roots of $b_{V_{\text {ind }}}(x)$ are contained in $[0,1)$; the claim follows.

\subsection{Compatibility of filtrations}

In this subsection, we are going to show a compatibility result between two filtrations on the $D_{W^{\prime}}$-module $\check{M}_{A^{\prime}}^{\beta}$ (recall that the $d \times(n+1)$ matrix $A^{\prime}$ has columns $\left.\underline{a}_{1}, \ldots, \underline{a}_{n}, \underline{a}_{1}+\cdots+\underline{a}_{n}\right)$. Let, as before, $F^{\text {ord }}$ be the filtration induced on $M_{A^{\prime}}^{\beta}$ by the filtration $F_{\bullet} D_{W^{\prime}}$ by the orders of differential operators. Moreover, let $V^{\bullet} D_{W^{\prime}}$ be the $V$-filtration on $D_{W^{\prime}}$ with respect to the coordinate $w_{n+1}$, and, as before, denote the induced filtration on $M_{A^{\prime}}^{\beta}$ by $V_{\text {ind }}^{\bullet} M_{A^{\prime}}^{\beta}$. Then the main result of this subsection can be stated as follows.

Proposition 4.9. Let $A$ be a $d \times n$ integer matrix, and suppose $\mathbb{N} A=\mathbb{Z}^{d} \cap \mathbb{R}_{\geqslant 0} A$ and $\mathbb{N} A \neq \mathbb{Z}^{d}$. Let $A^{\prime}:=\left(\underline{a}_{1}, \ldots, \underline{a}_{n}, \underline{a}_{1}+\cdots+\underline{a}_{n}\right)$, and consider the left $D_{W^{\prime} \text {-module }}$

$$
\check{M}_{A^{\prime}}^{\beta}=D_{W^{\prime}} /\left(\left(\check{\square}_{\underline{m}}\right)_{\underline{m} \in \mathbb{L}_{A^{\prime}}}+\left(\check{E}_{k}^{\prime}+\beta_{k}\right)_{k=1, \ldots, d}\right) .
$$

Then the map

$$
V^{k} D_{W^{\prime}} \cap F_{p} D_{W^{\prime}} \longrightarrow V_{\text {ind }}^{k} \check{M}_{A^{\prime}}^{\beta} \cap F_{p}^{\text {ord }} \check{M}_{A^{\prime}}^{\beta}
$$

is surjective.

The proof of this result will occupy this entire section. Before going into it, let us comment on how this result will enter in the calculation of the Hodge filtration on $\check{M}_{A^{\prime}}^{\beta}$. As will be explained in more detail at the beginning of Subsection 4.3, we consider the mixed Hodge module $h_{A *}{ }^{p} \mathbb{C}_{T}^{\beta, H}$ with underlying $\mathcal{D}_{W}$-module $h_{A+} \mathcal{O}_{T}^{\beta}$. If $A$ and $\beta$ satisfy the assumptions of Proposition 3.11, then this $\mathcal{D}_{W}$-module is $\check{\mathcal{M}}_{A}^{\beta}$. In order to compute the Hodge filtration on its module of global 


\section{Hypergeometric Hodge modules}

sections $\check{M}_{A}^{\beta}$, we will first consider the module $\check{M}_{A^{\prime}}^{\beta}=\Gamma\left(W^{\prime}, h_{A^{\prime}+}^{\beta} \mathcal{O}_{T}^{\beta}\right)$ and compute the Hodge filtration on it. We will use the fact that the embedding $h_{A^{\prime}}: T \hookrightarrow W^{\prime}$ can be factored as

$$
T \longrightarrow W \times \mathbb{C}_{t}^{*} \stackrel{j}{\longrightarrow} W^{\prime}=W \times \mathbb{C}_{t},
$$

where the first morphism is a closed embedding and the second one is the canonical open embedding of $W \times \mathbb{C}_{t}^{*}$ into $W^{\prime}$. Then the main tool to compute the Hodge filtration on $\check{M}_{A^{\prime}}^{\beta}$ is the following formula of Saito (see formula (4.9) below). Let $\left(\mathcal{M}, F_{\bullet}^{H}\right)$ be any filtered $\mathcal{D}_{W \times \mathbb{C}_{t}^{*} \text {-module }}$ underlying a complex mixed Hodge module in $\operatorname{MHM}\left(W \times \mathbb{C}_{t}^{*}, \mathbb{C}\right)$. Then the direct image $j_{+} \mathcal{M}$ underlies a complex mixed Hodge module on $W^{\prime}$, and its Hodge filtration is given as

$$
F_{p}^{H} j_{+} \mathcal{M}=\sum_{i \geqslant 0} \partial_{t}^{i}\left(V^{0} j_{+} \mathcal{M} \cap j_{*}\left(F_{q}^{H} \mathcal{M}\right)\right)
$$

where $V^{\bullet} j_{+} \mathcal{M}$ denotes the canonical $V$-filtration on $j_{+} \mathcal{M}$ with respect to the divisor $\{t=0\}$. We are going to apply this formula for the case where $\mathcal{M}$ is the direct image of $\mathcal{O}_{T}^{\beta}$ under the map $T \rightarrow W \times \mathbb{C}_{t}^{*}$ (so that $j_{+} \mathcal{M}=\check{\mathcal{M}}_{A^{\prime}}^{\beta}$ ). Since this map is a closed immersion, we can explicitly calculate the Hodge filtration on this direct image; that is, it is given as the shifted order filtration for a cyclic presentation. Moreover, if $\beta$ satisfies the assumptions of Proposition 4.8, then we have $V^{k} \check{M}_{A^{\prime}}^{\beta}=V_{\text {ind }}^{k} \check{M}_{A^{\prime}}^{\beta}$ for all $k \in \mathbb{Z}$ (in particular, for $k=0$ ), so that we have to compute the intersection of the order filtration on $M_{A^{\prime}}^{\beta}$ with the induced $V$-filtration on that module. As we will see in Subsection 4.3, this is possible since these two filtrations satisfy the compatibility statement of Proposition 4.9. Its proof relies on the very specific structure of the hypergeometric ideal $\check{I}_{A^{\prime}}=\left(\left(\check{\square}_{\underline{m}}\right)_{\underline{m} \in \mathbb{L}_{A^{\prime}}}+\left(\check{E}_{k}+\beta_{k}\right)_{k=1, \ldots, d}\right) \subset D_{W^{\prime}}$ and uses non-commutative Gröbner basis techniques; a good reference for needed results is [SST00]. We will recall the main definitions for the reader's convenience.

For notational convenience, we rename the coordinate $t$ on $W^{\prime}$ to be $w_{n+1}$, that is, $\Gamma\left(W^{\prime}, \mathcal{O}_{W^{\prime}}\right)$ $=\mathbb{C}\left[w_{1}, \ldots, w_{n}, w_{n+1}\right]$. We work in the Weyl algebra $D_{W^{\prime}}=\mathbb{C}\left[w_{1}, \ldots, w_{n+1}\right]\left\langle\partial_{w_{1}}, \ldots, \partial_{w_{n+1}}\right\rangle$. Any operator $P \in D_{W^{\prime}}$ has the so-called normally ordered expression $P=\sum_{(\gamma, \delta)} c_{\gamma \delta} w^{\gamma} \partial_{w}^{\delta} \in$ $D_{W^{\prime}}$, where the sum runs over all pairs $(\gamma, \delta)$ in some finite subset of $\mathbb{N}^{2(n+1)}$.

First, we define partial orders on the set of monomials in $D_{W^{\prime}}, \mathbb{C}[w]:=\mathbb{C}\left[w_{1}, \ldots, w_{n+1}\right]$ and $\mathbb{C}[w, \xi]:=\mathbb{C}\left[w_{1}, \ldots, w_{n+1}, \xi_{1}, \ldots, \xi_{n+1}\right]$, respectively, by choosing the weight vector $(u, v) \in$ $\mathbb{Z}^{2(n+1)}$ with $u_{i}+v_{i} \geqslant 0$ (for $D_{W^{\prime}}$ and $\mathbb{C}[w, \xi]$ ) and the weight vector $u \in \mathbb{Z}^{n+1}$ (for $\mathbb{C}[w]$ ). This means that the variables $w_{i}$ have weight $u_{i}$ and the partial differentials $\partial_{w_{i}}$ and $\xi_{i}$ have weight $v_{i}$. The associated partial order on monomials in $D_{W^{\prime}}$ is defined as follows: if for two monomials $w^{\gamma} \partial_{w}^{\delta}$ and $w^{c} \partial_{w}^{d}$, we have $\sum_{i} u_{i} c_{i}+v_{i} d_{i}<\sum_{i} u_{i} \gamma_{i}+v_{i} \delta_{i}$, then by definition $w^{\gamma} \partial_{w}^{\delta}$ is larger than $w^{c} \partial_{w}^{d}$ and we write $w^{c} \partial_{w}^{d} \prec_{(u, v)} w^{\gamma} \partial_{w}^{\delta}$. The partial orders on monomials in $\mathbb{C}[w]$ and on monomials in $\mathbb{C}[w, \xi]$ are defined similarly. The weight vector $(u, v)$ induces increasing and decreasing filtrations on $D_{W^{\prime}}$ given, respectively, by

$$
F_{p}^{(u, v)} D_{W^{\prime}}=\left\{\sum_{\sum_{i} u_{i} \gamma_{i}+v_{i} \delta_{i} \leqslant p} c_{\gamma \delta} w^{\gamma} \partial_{w}^{\delta}\right\} \quad \text { and } \quad F_{(u, v)}^{p} D_{W^{\prime}}=\left\{\sum_{\sum_{i} u_{i} \gamma_{i}+v_{i} \delta_{i} \geqslant p} c_{\gamma \delta} w^{\gamma} \partial_{w}^{\delta}\right\} .
$$

We define the graded ring $\operatorname{Gr}_{\bullet}^{(u, v)} D_{W^{\prime}}:=\bigoplus_{p} F_{p}^{(u, v)} D_{W^{\prime}} / F_{p-1}^{(u, v)} D_{W^{\prime}}$ associated with the weight $(u, v)$. Notice that for $(u, v)=(0, \ldots, 0,1, \ldots, 1)$ (that is, the $w_{i}$ have weight 0 and the $\partial_{w_{i}}$ have weight 1$)$, the ascending filtration $F_{\bullet}^{(u, v)} D_{W^{\prime}}$ is the order filtration $F_{\bullet} D_{W}^{\prime}$, and for $(u, v)=(0, \ldots$, $0,-1,0, \ldots, 0,1)$, the descending filtration $F_{(u, v)}^{\bullet} D_{W^{\prime}}$ is the $V$-filtration with respect to $w_{n+1}$. 


\section{T. Reichelt And C. Sevenheck}

We get well-defined maps

$$
\begin{gathered}
\operatorname{in}_{(u, v)}: D_{W^{\prime}} \longrightarrow \operatorname{Gr}_{\bullet}^{(u, v)} D_{W^{\prime}}=\mathbb{C}[w, \xi], \\
P=\sum_{\gamma, \delta} c_{\gamma \delta} w^{\gamma} \partial_{w}^{\delta} \longmapsto \operatorname{in}_{(u, v)}(P):=\sum_{\sum_{i} u_{i} \gamma_{i}+v_{i} \delta_{i}=m} c_{\gamma \delta} w^{\gamma} \xi^{\delta},
\end{gathered}
$$

where $m:=\operatorname{ord}_{(u, v)}(P):=\max \left\{\sum_{i} u_{i} \gamma_{i}+v_{i} \delta_{i} \mid c_{\gamma \delta} \neq 0\right\}$, and

$$
\begin{aligned}
& \operatorname{in}_{u}: \mathbb{C}[w] \longrightarrow \operatorname{Gr}_{\bullet}^{u} \mathbb{C}[w]=\mathbb{C}[w], \\
& Q=\sum_{\gamma} c_{\gamma} w^{\gamma} \longmapsto \operatorname{in}_{u}(Q):=\sum_{\sum_{i} u_{i} \gamma_{i}=m} c_{\delta} w^{\delta},
\end{aligned}
$$

where $m=\max \left\{\sum_{u} u_{i} \gamma_{i} \mid c_{\gamma} \neq 0\right\}$, and

$$
\begin{aligned}
\operatorname{in}_{(u, v)}: \mathbb{C}[w, \xi] & \longrightarrow \operatorname{Gr}_{\bullet}^{(u, v)} \mathbb{C}[w, \xi]=\mathbb{C}[w, \xi], \\
R=\sum_{\gamma, \delta} c_{\gamma} w^{\gamma} \xi^{\delta} & \longrightarrow \operatorname{in}_{(u, v)}(Q):=\sum_{\sum_{i} u_{i} \gamma_{i}+v_{i} \delta_{i}=m} c_{\delta} w^{\delta},
\end{aligned}
$$

where $m:=\operatorname{ord}_{(u, v)}(R):=\max \left\{\sum_{i} u_{i} \gamma_{i}+v_{i} \delta_{i} \mid c_{\gamma \delta} \neq 0\right\}$. Notice that, in contrast to the case of a total ordering, the initial terms $\operatorname{in}_{(u, v)}$ and in $_{u}$ are in general not monomials.

Let $I^{\prime} \subset D_{W^{\prime}}$ be a left ideal. The set $\operatorname{in}_{(u, v)}\left(I^{\prime}\right)$ is an ideal in $\operatorname{Gr}_{\bullet}^{(u, v)} D_{W^{\prime}}$ and is called the initial ideal of $I^{\prime}$ with respect to the weight vector $(u, v)$. A finite subset $G$ of $D_{W^{\prime}}$ is a Gröbner basis of $I^{\prime}$ with respect to $(u, v)$ if $I^{\prime}$ is generated by $G$ and $\operatorname{in}_{(u, v)}\left(I^{\prime}\right)$ is generated by $\operatorname{in}_{(u, v)}(G)$. Similarly, let $J^{\prime} \subset \mathbb{C}[w]$ and $K^{\prime} \subset \mathbb{C}[w, \xi]$ be ideals. The set $\operatorname{in}_{u}\left(J^{\prime}\right)$ is an ideal in $\operatorname{Gr}_{\bullet}^{u} \mathbb{C}[w]$ and is called the initial ideal of $J^{\prime}$ with respect to the weight vector $u$. The set $\operatorname{in}_{(u, v)}\left(K^{\prime}\right)$ is an ideal in $\operatorname{Gr}_{\bullet}^{(u, v)} \mathbb{C}[w, \xi]$ and is called the initial ideal of $K^{\prime}$ with respect to the weight vector $(u, v)$. The definition of a Gröbner basis is parallel to the definition above.

Let $\check{I}_{A^{\prime}}=\left(\left(\check{\square}_{\underline{m}}\right)_{\underline{m} \in \mathbb{L}_{A^{\prime}}}+\left(\check{E}_{k}^{\prime}+\beta_{k}\right)_{k=1, \ldots, d}\right)$ be the hypergeometric ideal. The fake initial ideal $\operatorname{fin}_{(u, v)}\left(\check{I}_{A^{\prime}}\right)$ is the following ideal in $\operatorname{Gr}_{(u, v)} D_{W^{\prime}}$ :

$$
\operatorname{fin}_{(u, v)}\left(\check{I}_{A^{\prime}}\right):=\operatorname{Gr}_{(u, v)} \mathcal{D}_{W^{\prime}} \cdot \operatorname{in}_{u}\left(\check{J}_{A^{\prime}}\right)+\sum_{k=1}^{d} \operatorname{Gr}_{(u, v)} \mathcal{D}_{W^{\prime}} \cdot \operatorname{in}_{(u, v)}\left(\check{E}_{k}+\beta_{k}\right),
$$

where $\check{J}_{A^{\prime}} \subset \mathbb{C}[w]$ is the ideal generated by $\left(\check{\square}_{\underline{m}}\right)_{\underline{m} \in \mathbb{L}_{A^{\prime}}}$.

Consider the Koszul complex

$$
\cdots \stackrel{d_{-2}}{\longrightarrow} K^{-1}\left(\operatorname{Gr}_{\bullet}^{(u, v)}\left(D_{W^{\prime}} / D_{W^{\prime}} \check{J}_{A^{\prime}}\right)\right) \stackrel{d_{-1}}{\longrightarrow} K^{0}\left(\mathrm{Gr}_{\bullet}^{(u, v)}\left(D_{W^{\prime}} / D_{W^{\prime}} \check{J}_{A^{\prime}}\right)\right) \longrightarrow 0,
$$

where

$$
K^{-p}\left(\operatorname{Gr}_{\bullet}^{(u, v)}\left(D_{W^{\prime}} / D_{W^{\prime}} \check{J}_{A^{\prime}}\right)\right)=\bigoplus_{1 \leqslant i_{1}<\cdots<i_{p} \leqslant s+1} \operatorname{Gr}_{\bullet}^{(u, v)}\left(D_{W^{\prime}} / D_{W^{\prime}} \check{J}_{A^{\prime}}\right) e_{i_{1} \ldots i_{p}}
$$

and

$$
d_{-p}\left(e_{i_{1} \ldots i_{p}}\right)=\sum_{k=1}^{p}(-1)^{k-1} \operatorname{in}_{(u, v)}\left(\check{E}_{k}+\beta_{k}\right) e_{i_{1} \ldots \widehat{i}_{k} \ldots i_{p}} .
$$

The following statement is an easy adaption of [SST00, Theorem 4.3.5].

Proposition 4.10. If the cohomology $H^{-1}\left(K^{\bullet}\left(\mathrm{Gr}_{\bullet}^{(u, v)}\left(D_{W^{\prime}} / D_{W^{\prime}} \check{J}_{A^{\prime}}\right)\right)\right)$ vanishes, then the initial ideal satisfies $\operatorname{in}_{(u, v)}\left(\check{I}_{A^{\prime}}\right)=\operatorname{fin}_{(u, v)}\left(\check{I}_{A^{\prime}}\right)$. 


\section{Hypergeometric Hodge modules}

Proof. After a Fourier-Laplace transform $w_{i} \mapsto \partial_{x_{i}}$ and $\partial_{w_{i}} \mapsto-x_{i}$, the proof carries over word for word from loc. cit. (Notice that in Chapter 4 of [SST00], the homogenity of $A^{\prime}$ is assumed; however, the proof of this statement does not need this requirement.)

Recall that $A^{\prime}$ is a matrix built from the matrix $A$ by adding a column which is the sum over all columns of $A$. Let $\check{J}_{A} \subset \mathbb{C}\left[w_{1}, \ldots, w_{n}\right]$ be the ideal generated by $\left(\check{\square}_{l}\right)_{l \in \mathbb{L}_{A}}$. We choose generators $g_{1}, \ldots, g_{\ell-1}$ of $\breve{J}_{A}$. Notice that $g_{1}, \ldots, g_{\ell-1}, g_{\ell}:=w_{n+1}-w_{1} \cdot \ldots \cdot w_{n}$ is a basis of $\breve{J}_{A^{\prime}} \subset \mathbb{C}\left[w_{1}, \ldots, w_{n+1}\right]$.

LEMma 4.11. The elements $g_{1}, \ldots, g_{\ell}$ form a Gröbner basis of $\check{J}_{A^{\prime}}$ with respect to the weight vector $(0, \ldots, 0,-e)$ with $e>0$.

Proof. We have already seen that $g_{1}, \ldots, g_{\ell}$ is a basis of $\check{J}_{A^{\prime}}$. It remains to prove that $\operatorname{in}_{(0, \ldots, 0,-e)}\left(g_{1}\right)$ $=g_{1}, \ldots, \operatorname{in}_{(0, \ldots, 0,-e)}\left(g_{\ell-1}\right)=g_{\ell-1}, \operatorname{in}_{(0, \ldots, 0,-e)}\left(g_{\ell}\right)=w_{1} \cdot \ldots \cdot w_{n}$ is a basis of $\operatorname{in}_{(0, \ldots, 0,-e)}\left(\breve{J}_{A^{\prime}}\right)$. Let

$$
x=\sum_{i=1}^{\ell} x_{i} g_{i}
$$

and $-e \cdot N:=\max \left\{\operatorname{ord}_{(0, \ldots, 0,-e)}\left(x_{i} g_{i}\right) \mid i=1, \ldots, \ell\right\}$. Assume $\operatorname{ord}_{(0, \ldots, 0,-e)}(x)<-e \cdot N$; then the maximal $w_{n+1}$-degree component of the equation (4.5) is given by

$$
0=\sum_{i=1}^{\ell-1} w_{n+1}^{N} p_{i} g_{i}+w_{n+1}^{N} p_{\ell} \cdot\left(w_{1} \cdot \ldots \cdot w_{n}\right)
$$

for polynomials $p_{i} \in \mathbb{C}\left[w_{1}, \ldots, w_{n}\right]$. Since $\check{J}_{A}=\left(g_{1}, \ldots, g_{\ell-1}\right)$ is a prime ideal and $w_{1} \cdot \ldots \cdot w_{n} \notin$ $\check{J}_{A}$, we conclude that $p_{\ell} \in \check{J}_{A}$. Hence, there exist polynomials $q_{i} \in \mathbb{C}\left[w_{1}, \ldots, w_{n}\right]$ such that $p_{\ell}=\sum_{i=1}^{\ell-1} q_{i} g_{i}$. We get

$$
x=\sum_{i=1}^{\ell} x_{i} g_{i}-\sum_{i=1}^{\ell-1} w_{n+1}^{N} p_{i} g_{i}-w_{n+1}^{N} p_{\ell} \cdot g_{\ell}+w_{n+1}^{N+1}\left(\sum_{i=1}^{\ell-1} q_{i} g_{i}\right)=\sum_{i=1}^{\ell} x_{i}^{\prime} g_{i}
$$

for $x_{i}^{\prime} \in \mathbb{C}\left[w_{1}, \ldots, w_{n+1}\right]$ with $\max \left\{\operatorname{ord}_{(0, \ldots, 0,-e)}\left(x_{i}^{\prime} g_{i}\right) \mid i=1, \ldots, \ell\right\}<-e \cdot N$. By induction, we can reduce to the case $\operatorname{ord}_{(0, \ldots, 0,-e)}(x)=-e \cdot N$. In this case, for the maximal $w_{n+1}$-degree component, we get

$$
\begin{aligned}
\operatorname{in}_{(0, \ldots, 0,-e)}(x) & =\sum w_{n+1}^{N} p_{i}^{\prime} g_{i}+w_{n+1}^{N} p_{\ell}^{\prime} \cdot\left(w_{1} \cdot \ldots \cdot w_{n}\right) \\
& =\sum w_{n+1}^{N} p_{i}^{\prime} \operatorname{in}_{(0, \ldots, 0,-e)}\left(g_{i}\right)+w_{n+1}^{N} p_{\ell}^{\prime} \operatorname{in}_{(0, \ldots, 0,-e)}\left(g_{\ell}\right)
\end{aligned}
$$

for polynomials $p_{i}^{\prime} \in \mathbb{C}\left[w_{1}, \ldots, w_{n}\right]$. This shows the claim.

Proposition 4.12. Let $A$ be a $d \times n$ integer matrix such that $\mathbb{N} A=\mathbb{R}_{\geqslant 0} A \cap \mathbb{Z}^{d}$ and $\mathbb{N} A \neq \mathbb{Z}^{d}$. Let $A^{\prime}$ be the matrix built from $A$ by adding a column which is the sum over all columns of $A$. Then

$$
\operatorname{fin}_{(u, v)}\left(\check{I}_{A^{\prime}}\right)=\operatorname{in}_{(u, v)}\left(\check{I}_{A^{\prime}}\right)
$$

if

(i) $(u, v)=(0,0, \ldots, 0,1,1, \ldots, 1)$,

(ii) $(u, v)=(0, \ldots, 0,-e, 1, \ldots, 1,1+e)$ for $0<e<1$.

Proof. The first case was proven in [SST00, Corollary 4.36] for homogeneous $A$. In order to prove the statement for $(u, v)=(0,0, \ldots, 0,1,1, \ldots, 1)$ in the general case, we first observe that 


\section{T. Reichelt And C. Sevenheck}

$\operatorname{Gr}_{(v, u)}\left(D_{W^{\prime}} / \check{J}_{A^{\prime}}\right)$ is isomorphic to

$$
\mathbb{C}\left[\xi_{1}, \ldots, \xi_{n+1}\right] \otimes_{\mathbb{C}} \mathbb{C}\left[\mathbb{N} A^{\prime}\right],
$$

which is Cohen-Macaulay by the assumption $\mathbb{N} A=\mathbb{R}_{\geqslant 0} A \cap \mathbb{Z}^{d}$ and the fact that $\mathbb{N} A=\mathbb{N} A^{\prime}$ as well as $\mathbb{R}_{\geqslant 0} A=\mathbb{R}_{\geqslant 0} A^{\prime}$. It follows from [BGM15, Theorem 1.2] that the elements $\operatorname{in}_{(u, v)}\left(\check{E}_{k}+\beta_{k}\right)$ are part of a system of parameters in $\mathbb{C}\left[\xi_{1}, \ldots, \xi_{n+1}\right] \otimes_{\mathbb{C}} \mathbb{C}\left[\mathbb{N} A^{\prime}\right]$, and since this ring is CohenMacaulay, they also form a regular sequence. Therefore, $H^{-1}\left(K^{\bullet}\left(\operatorname{Gr}_{(u, v)}\left(D_{W^{\prime}} / D_{W^{\prime}} \check{J}_{A^{\prime}}\right)\right)\right)=0$, and the claim follows from Proposition 4.10.

We prove the second claim. Since $\mathbb{N} A \neq \mathbb{Z}^{d}$ holds, the last column of $A^{\prime}$, which is the sum of the columns of $A$, is non-zero (this was shown above Lemma 4.4). Hence, we can assume (by elementary row manipulations of $A^{\prime}$, which do not change the ideal $\check{I}_{A^{\prime}}$ ) that the last column of $A^{\prime}$ is zero except for the entry in the first row. Set

$$
\check{e}_{k}:=\sum_{i=1}^{n} a_{k i} w_{i} \xi_{i} \quad \text { for } \quad k=1, \ldots, d .
$$

We will use the generators $g_{1}, \ldots, g_{\ell-1}$ of $\check{J}_{A}$ from Lemma 4.11. It follows from [BGM15, Theorem 1.2] that $\check{e}_{1}, \ldots, \check{e}_{d}$ is part of a system of parameters for

$$
\begin{aligned}
\mathbb{C}\left[\xi_{1}, \ldots, \xi_{n}\right] \otimes_{\mathbb{C}} \mathbb{C}[\mathbb{N} A] & \simeq \mathbb{C}\left[\xi_{1}, \ldots, \xi_{n}, w_{1}, \ldots, w_{n}\right] / \mathbb{C}\left[\xi_{1}, \ldots, \xi_{n}, w_{1}, \ldots, w_{n}\right] \check{J}_{A} \\
& \simeq \mathbb{C}\left[\xi_{1}, \ldots, \xi_{n}, w_{1}, \ldots, w_{n}\right] /\left(g_{1}, \ldots, g_{\ell-1}\right),
\end{aligned}
$$

where $\mathbb{C}[\mathbb{N} A]$ has Krull dimension $d$. Therefore,

$$
\mathbb{C}\left[\xi_{1}, \ldots, \xi_{n}, w_{1}, \ldots, w_{n}\right] /\left(g_{1}, \ldots, g_{\ell-1}, \check{e}_{1}, \ldots, \check{e}_{r}\right)
$$

has Krull dimension $n$.

We will show that the Krull dimension of

$$
\mathbb{C}\left[\xi_{1}, \ldots, \xi_{n}, w_{1}, \ldots, w_{n}\right] /\left(g_{1}, \ldots, g_{\ell-1}, w_{1} \cdot \ldots \cdot w_{n}, \check{e}_{2}, \ldots, \check{e}_{r}\right)
$$

is also $n$ (notice that we omitted $\check{e}_{1}$ ). The variety corresponding to $\mathbb{C}\left[\xi_{1}, \ldots, \xi_{n}\right] \otimes_{\mathbb{C}} \mathbb{C}[\mathbb{N} A]$ is

$$
\mathbb{C}^{n} \times X_{A} \subset \mathbb{C}^{n} \times \mathbb{C}^{n}
$$

where $X_{A}:=\operatorname{Spec} \mathbb{C}[\mathbb{N} A]$. The toric variety $X_{A}$ is a finite disjoint union of torus orbits, where the big dense torus lies in $\left\{w_{1} \cdot \ldots \cdot w_{n} \neq 0\right\}$ and the smaller-dimensional tori lie in $\left\{w_{1} \cdot \ldots \cdot w_{n}=0\right\}$. Hence,

$$
\mathbb{C}\left[\xi_{1}, \ldots, \xi_{n}, w_{1}, \ldots, w_{n}\right] /\left(g_{1}, \ldots, g_{\ell-1}, w_{1} \cdot \ldots \cdot w_{n}\right)
$$

has Krull dimension $n+d-1$. The torus orbits of $X_{A}$ correspond to the faces of the cone $\mathbb{R}_{\geqslant 0} A$, where the big dense torus corresponds to $\mathbb{R}_{\geqslant 0} A$ itself. For a face $\tau \subsetneq \mathbb{R}_{\geqslant 0} A$, the torus orbit $\operatorname{Orb}(\tau)$ is given by $\operatorname{Orb}(\tau)=X_{A} \cap\left(\mathbb{C}_{w}^{*}\right)^{\tau}$, where $\left(\mathbb{C}^{*}\right)_{w}^{\tau}=\left\{w \in \mathbb{C}^{n} \mid w_{i}=0\right.$ for $a_{i} \notin \tau, w_{j} \neq 0$ for $\left.a_{j} \in \tau\right\}$. Hence, it suffices to prove that $\left(\mathbb{C}^{n} \times \operatorname{Orb}(\tau)\right) \cap V\left(\left(\check{e}_{2}, \ldots, \check{e}_{d}\right)\right)$ has dimension $n$, where $V\left(\left(\check{e}_{2}, \ldots, \check{e}_{d}\right)\right)$ is the vanishing locus of the ideal generated by $\check{e}_{2}, \ldots, \check{e}_{d}$. Set $\mathbb{C}_{\xi}^{\tau}=\left\{\xi \in \mathbb{C}^{n} \mid\right.$ $\xi_{i}=0$ for $\left.a_{i} \notin \tau\right\}$. It is enough to show that $\mathbb{C}_{\xi}^{\tau} \times \operatorname{Orb}(\tau) \cap V\left(\left(\check{e}_{2}^{\tau}, \ldots, \check{e}_{d}^{\tau}\right)\right)$ has dimension at most $\sharp\left\{i \mid a_{i} \in \tau\right\}$, where $\check{e}_{k}^{\tau}:=\sum_{i: a_{i} \in \tau} a_{k i} w_{i} \xi_{i}$. The codimension of $V\left(\left(\check{e}_{2}^{\tau}, \ldots, \check{e}_{d}^{\tau}\right)\right)$ is $\operatorname{dim}(\tau)$ since $(1,0, \ldots, 0)=(1 / c)\left(a_{1}+\cdots+a_{n}\right)$ (for a suitable $\left.c \in \mathbb{Z} \backslash\{0\}\right)$ lies in the interior of $\mathbb{R}_{\geqslant 0} A$, hence not in $\tau$, and therefore the matrix $\left(a_{k i}\right)_{k \geqslant 2, i: a_{i} \in \tau}$ has $\operatorname{rank} \operatorname{dim}(\tau)$. By [BGM15, Lemma 1.1], the intersection of $\mathbb{C}_{\xi}^{\tau} \times \operatorname{Orb}(\tau)$ with $V\left(\left(\check{e}_{2}^{\tau}, \ldots, \check{e}_{d}^{\tau}\right)\right)$ is transverse. Since the codimension of $V\left(\left(\check{e}_{2}^{\tau}, \ldots, \check{e}_{d}^{\tau}\right)\right)$ is $\operatorname{dim} \operatorname{Orb}(\tau)=\operatorname{dim}(\tau)$, the intersection has dimension $\sharp\left\{i \mid a_{i} \in \tau\right\}$. This shows that the Krull dimension of (4.6) is $n$. 


\section{Hypergeometric Hodge modules}

Let

$$
\begin{aligned}
& \check{e}_{1}^{\prime}:=\check{e}_{1}+\left(\sum_{i=1}^{n} a_{1 i}\right) x_{n+1} \xi_{n+1}=\operatorname{in}_{(0, \ldots, 0,-e, 1, \ldots, 1,1+e)}\left(\check{E}_{1}^{\prime}+\beta_{1}\right), \\
& \check{e}_{k}^{\prime}:=\check{e}_{k}=\operatorname{in}_{(0, \ldots, 0,-e, 1, \ldots, 1,1+e)}\left(\check{E}_{k}^{\prime}+\beta_{k}\right) \quad \text { for } \quad k=2, \ldots, d
\end{aligned}
$$

and

$$
\begin{aligned}
& \bar{g}_{i}:=\operatorname{in}_{(0, \ldots, 0,-e, 1, \ldots, 1,1+e)}\left(g_{i}\right)=g_{i} \text { for } i=1, \ldots, \ell-1, \\
& \bar{g}_{\ell}:=\operatorname{in}_{(0, \ldots, 0,-e, 1, \ldots, 1,1+e)}\left(g_{\ell}\right)=w_{1} \cdot \ldots \cdot w_{n} .
\end{aligned}
$$

Since $\bar{g}_{1}, \ldots, \bar{g}_{\ell}$ and $\check{e}_{2}^{\prime}, \ldots, \check{e}_{d}^{\prime}$ are independent of $w_{n+1}, \xi_{n+1}$ and $\check{e}_{1}^{\prime}=\check{e}_{1}+\left(\sum_{i=1}^{n} a_{1 i}\right) x_{n+1} \xi_{n+1}$ is (for degree reasons) a nonzerodivisor on

$$
\mathbb{C}\left[w_{n+1}, \xi_{n+1}\right] \otimes_{\mathbb{C}} \mathbb{C}\left[\xi_{1}, \ldots, \xi_{n}, w_{1}, \ldots, w_{n}\right] /\left(\bar{g}_{1}, \ldots, \bar{g}_{\ell}, \check{e}_{2}, \ldots, \check{e}_{d}\right),
$$

one easily sees that

$$
\mathbb{C}\left[w_{1}, \ldots, w_{n+1}, \xi_{1}, \ldots, \xi_{n+1}\right] /\left(\bar{g}_{1}, \ldots, \bar{g}_{\ell}, \check{e}_{1}^{\prime}, \ldots, \check{e}_{d}^{\prime}\right)
$$

has Krull dimension $n+1$. It follows from $(4.7)$ that $\mathbb{C}\left[w_{1}, \ldots, w_{n+1}, \xi_{1}, \ldots, \xi_{n+1}\right] /\left(\bar{g}_{1}, \ldots, \bar{g}_{\ell}\right)$ has Krull dimension $n+d+1$; hence, $\check{e}_{1}^{\prime}, \ldots, \check{e}_{d}^{\prime}$ is part of a system of parameters. By the assumption on $A$, the ring

$$
\mathbb{C}\left[w_{1}, \ldots, w_{n+1}, \xi_{1}, \ldots, \xi_{n+1}\right] /\left(\bar{g}_{1}, \ldots, \bar{g}_{\ell-1}\right) \simeq \mathbb{C}\left[w_{n+1}, \xi_{1}, \ldots, \xi_{n+1}\right] \otimes_{\mathbb{C}} \mathbb{C}[\mathbb{N} A]
$$

is Cohen-Macaulay. Since $\bar{g}_{\ell}=w_{1} \cdot \ldots \cdot w_{n}$ is not a zero divisor in the ring above (because $\mathbb{C}[\mathbb{N} A]$ has no non-zero zero divisors), we see that the ring

$$
\mathbb{C}\left[w_{1}, \ldots, w_{n+1}, \xi_{1}, \ldots, \xi_{n+1}\right] /\left(\bar{g}_{1}, \ldots, \bar{g}_{\ell}\right)
$$

is also Cohen-Macaulay, and therefore $\check{e}_{1}^{\prime}, \ldots, \check{e}_{d}^{\prime}$ is a regular sequence in

$$
\mathbb{C}\left[w_{1}, \ldots, w_{n+1}, \xi_{1}, \ldots, \xi_{n+1}\right] /\left(\bar{g}_{1}, \ldots, \bar{g}_{\ell}\right) .
$$

Since

$$
\operatorname{Gr}_{(0, \ldots, 0,-e, 1, \ldots, 1,1+e)}\left(D_{W^{\prime}} / D_{W^{\prime}} \check{J}_{A^{\prime}}\right) \simeq \mathbb{C}\left[w_{1}, \ldots, w_{n+1}, \xi_{1}, \ldots, \xi_{n+1}\right] /\left(\bar{g}_{1}, \ldots, \bar{g}_{\ell}\right)
$$

and $\check{e}_{k}^{\prime}=\operatorname{in}_{(0, \ldots, 0,-e, 1, \ldots, 1,1+e)}\left(\check{E}_{k}^{\prime}+\beta_{k}\right)$ for $k=1, \ldots, d$, we have

$$
H^{-1}\left(K^{\bullet}\left(\operatorname{Gr}_{(0, \ldots, 0,-e, 1, \ldots, 1,1+e)}\left(D_{W^{\prime}} / D_{W^{\prime}} \check{J}_{A^{\prime}}\right)\right)\right)=0 .
$$

Using Proposition 4.10 again, this shows the second claim.

Corollary 4.13. Let $g_{1}, \ldots, g_{\ell} \in \mathbb{C}\left[w_{1}, \ldots, w_{n+1}\right]$ be the generators of $\check{J}_{A^{\prime}}$ defined above Lemma 4.11.

(i) The $\left(g_{i}\right)_{i=1, \ldots, \ell}$ together with $\left(\check{E}_{k}^{\prime}+\beta_{k}\right)_{k=1, \ldots, d}$ form a Gröbner basis of $\check{I}_{A^{\prime}}$ with respect to the weight vector $(u, v)=(0, \ldots, 0,1, \ldots, 1)$.

(ii) Let $(u, v)=(0, \ldots, 0,1, \ldots, 1)$, and set $\tilde{g}_{i}:=\operatorname{in}_{(u, v)}\left(g_{i}\right)$ and $\tilde{E}_{k}^{\prime}=\operatorname{in}_{(u, v)}\left(\check{E}_{k}^{\prime}+\beta_{k}\right)$. The elements $\left(\tilde{g}_{i}\right)_{i=1, \ldots, \ell}$ and $\left(\tilde{E}_{k}^{\prime}\right)_{k=1, \ldots, d}$ form a Gröbner basis of

$$
\begin{aligned}
\operatorname{in}_{(u, v)}\left(\check{I}_{A^{\prime}}\right) & =\operatorname{in}_{(u, v)}\left(\left(g_{1}, \ldots, g_{\ell}, \check{E}_{1}^{\prime}+\beta_{1}, \ldots, \check{E}_{d}^{\prime}+\beta_{d}\right)\right) \\
& =\left(\tilde{g}_{1}, \ldots, \tilde{g}_{\ell}, \tilde{E}_{1}^{\prime}, \ldots, \tilde{E}_{d}^{\prime}\right) \subset \mathbb{C}\left[w_{1}, \ldots, w_{n+1}, \xi_{1}, \ldots, \xi_{n+1}\right]
\end{aligned}
$$

with respect to the weight vector $(0, \ldots, 0,-1,0, \ldots, 0,1)$ (that is, $w_{n+1}$ has weight -1 and $\xi_{n+1}$ has weight +1$)$. 


\section{T. Reichelt And C. Sevenheck}

Proof. (i) The set $\left(g_{i}\right)_{i=1, \ldots, \ell}$ is a Gröbner basis for $\check{J}_{A^{\prime}}$. Therefore, the elements $\operatorname{in}_{u}\left(g_{i}\right)=g_{i}$ generate $\operatorname{in}_{u}\left(\check{J}_{A^{\prime}}\right)=\breve{J}_{A^{\prime}}$. The elements $\left(g_{i}\right)_{i=1, \ldots, \ell}$ and $\left(\check{E}_{k}+\beta_{k}\right)_{k=1, \ldots, d}$ generate $\check{I}_{A^{\prime}}$, and the elements $\left(\operatorname{in}_{u, v}\left(g_{i}\right)\right)_{i=1, \ldots, \ell}$ and $\left(\operatorname{in}_{(u, v)}\left(\check{E}_{k}+\beta_{k}\right)\right)_{k=1, \ldots, d}$ generate fin $(u, v)$ by definition. The claim now follows from Proposition 4.12(i).

(ii) It follows from the first point that the $\tilde{g}_{i}=\operatorname{in}_{(u, v)}\left(g_{i}\right)$ and the $\tilde{E}_{k}^{\prime}=\operatorname{in}_{(u, v)}\left(\check{E}_{k}^{\prime}+\beta_{k}\right)$ generate $\operatorname{in}_{(u, v)}\left(\check{I}_{A^{\prime}}\right)$. We have to show that the $\operatorname{in}_{(0, \ldots, 0,-1,0, \ldots, 0,1)}\left(\tilde{g}_{i}\right)$ for $i=1, \ldots, d$ and the $\operatorname{in}_{(0, \ldots, 0,-1,0, \ldots, 0,1)}\left(\tilde{E}_{k}^{\prime}\right)$ generate $\operatorname{in}_{(0, \ldots, 0,-1,0, \ldots, 0,1)}\left(\operatorname{in}_{(u, v)}\left(\check{I}_{A^{\prime}}\right)\right)$. But this follows from (cf. [SST00, Lemma 2.1.6(2)])

$$
\begin{aligned}
\operatorname{in}_{(0, \ldots, 0,-1,0, \ldots, 0,1)}\left(\tilde{g}_{i}\right) & =\operatorname{in}_{(0, \ldots, 0,-e, 1, \ldots, 1,1+e)}\left(g_{i}\right)
\end{aligned} \quad \text { for } \quad i=1, \ldots, \ell,
$$

for $0<e \ll 1$ and Proposition 4.12(ii).

The second notion we are going to introduce relates the order filtration $F_{\bullet}$ on $D_{W^{\prime}}$ with the $V$-filtration that already occurred in Subsection 4.1. Here we consider the descending $V$-filtration on $D_{W^{\prime}}$ with respect to $w_{n+1}=0$, which we again denote by $V^{\bullet} D_{W^{\prime}}$. We have

$$
V^{0} D_{W^{\prime}}=\sum_{i, k \geqslant 0}\left(\partial_{w_{n+1}} w_{n+1}\right)^{i}\left(w_{n+1}\right)^{k} P_{i}
$$

for $P_{i} \in \mathbb{C}\left[w_{1}, \ldots, w_{n}\right]\left\langle\partial_{w_{1}}, \ldots, \partial_{w_{n}}\right\rangle$ and

$$
V^{k} D_{W^{\prime}}=w_{n+1}^{k} V^{0} D_{W^{\prime}} \quad \text { and } \quad V^{-k} D_{W^{\prime}}=\sum_{j \geqslant 0} \partial_{w_{n+1}}^{j} V^{0} D_{W^{\prime}}
$$

for $k>0$.

Recall the left ideal $\check{I}_{A^{\prime}} \subset D_{W^{\prime}}$ and the left $D_{W^{\prime}}$-modules $\check{M}_{A^{\prime}}^{\beta}:=D_{W^{\prime}} / \check{I}_{A^{\prime}}$ from above. We define filtrations $V_{\text {ind }}^{\bullet}$ and $F_{\bullet}^{\text {ord }}$ on $\check{M}_{A^{\prime}}^{\beta}$ by

$$
V_{\text {ind }}^{k} \check{M}_{A^{\prime}}^{\beta}:=\frac{V^{k} D_{W^{\prime}}+\check{I}_{A^{\prime}}}{\check{I}_{A^{\prime}}} \text { and } F_{p}^{\text {ord }} \check{M}_{A^{\prime}}^{\beta}:=\frac{F_{p} D_{W^{\prime}}+\check{I}_{A^{\prime}}}{\check{I}_{A^{\prime}}} \text {. }
$$

We are now ready to prove the main result of this subsection.

Proof of Proposition 4.9. Let $m \in V_{\text {ind }}^{k} \check{M}_{A^{\prime}}^{\beta} \cap F_{p}^{\text {ord }} \check{M}_{A^{\prime}}^{\beta}$. We can find $P, Q \in D_{W^{\prime}}$ such that $P \in F_{p} D_{W^{\prime}}, Q \in V^{k} D_{W^{\prime}}$ and $[P]=m=[Q]$; that is, $P=Q-i$ for some $i \in \check{I}_{A^{\prime}}$. We have to find a $Q^{\prime}$ with $Q^{\prime} \in V^{k} D_{W^{\prime}} \cap F_{p} D_{W^{\prime}}$ with $P=Q^{\prime}-i^{\prime}$ for $i^{\prime} \in I$. We will construct this element $Q^{\prime}$ by decreasing induction on the order of $Q$ by killing its leading term in each step. For this, we will use the special Gröbner basis of $\check{I}_{A^{\prime}}$ which we constructed in Corollary 4.13.

Recall that the weight vector $(u, v):=(0, \ldots, 0,1, \ldots, 1)$ induces the order filtration $F_{\bullet}^{(u, v)}=$ $F_{\bullet}^{\text {ord }}$ on $D_{W^{\prime}}$. If $R \in D_{W^{\prime}}$ and $k:=\operatorname{ord}_{(u, v)}(R)$, we define the symbol of $R$ by $\sigma_{k}(R)=\operatorname{in}_{(u, v)}(R)$ and set $\sigma_{q}(R)=0$ for $q \neq k$. We define a second weight vector $\left(u^{\prime}, v^{\prime}\right):=(0, \ldots, 0,-1,0, \ldots, 0,1)$ which induces the descending $V$-filtration from (4.8) on $D_{W^{\prime}}$. The $V$-filtration and $F$-filtration also induce filtrations $\tilde{V}$ and $\tilde{F}$ on $\operatorname{Gr}_{\bullet}^{(u, v)} D_{W^{\prime}}=\operatorname{Gr}_{\bullet}^{F} D_{W^{\prime}}=\mathbb{C}\left[w_{1}, \ldots, w_{n+1}, \xi_{1}, \ldots, \xi_{n+1}\right]$.

Let $t_{Q}:=\operatorname{ord}_{(u, v)} Q, t_{i}:=\operatorname{ord}_{(u, v)} i$, and set $t:=\max \left(t_{Q}, t_{i}\right)$. Obviously, we have $t \geqslant p$. If $t=p$, we are done. Hence, we assume $t>p$; thus, we have

$$
0=\sigma_{t}(P)=\sigma_{t}(Q-i)
$$




\section{HypergeOMetric Hodge MOdules}

and therefore $t=t_{Q}=t_{i}$, which implies $\sigma_{t}(Q)=\sigma_{t}(i) \neq 0$. Set $k_{Q}:=\operatorname{ord}_{\left(u^{\prime}, v^{\prime}\right)}\left(\sigma_{t}(Q)\right)$; then we have $\sigma_{t}(i)=\sigma_{t}(Q) \in \tilde{V}^{k_{Q}}$.

Recall from Corollary 4.13 that $\check{I}_{A^{\prime}}$ is generated by $\left\{G_{1}, \ldots, G_{m}\right\}:=\left\{g_{1}, \ldots, g_{\ell}, \check{E}_{1}^{\prime}+\beta_{1}, \ldots\right.$, $\left.\check{E}_{d}^{\prime}+\beta_{d}\right\}$, that these elements form a Gröbner basis with respect to the weight vector $(u, v)$ and that their initial forms

$$
\left\{\tilde{G}_{1}, \ldots, \tilde{G}_{m}\right\}:=\left\{\operatorname{in}_{(u, v)}\left(G_{1}\right), \ldots, \operatorname{in}_{(u, v)}\left(G_{m}\right)\right\}
$$

are a Gröbner basis of $\operatorname{in}_{(u, v)}\left(\check{I}_{A^{\prime}}\right)$ with respect to the weight vector $\left(u^{\prime}, v^{\prime}\right)$. Therefore, we can write

$$
\sigma_{t}(i)=\sum_{l=1}^{m} \tilde{i}_{l} \tilde{G}_{l}
$$

with $\tilde{i}_{l} \in \mathbb{C}\left[w_{1}, \ldots, w_{n+1}, \xi_{1}, \ldots, \xi_{n+1}\right]$. Using a commutative version of [SST00, Theorem 1.2.10], we can assume $\tilde{i}_{l} \in \tilde{V}^{k_{Q}-k_{l}}$, where $k_{l}=\operatorname{ord}_{\left(u^{\prime}, v^{\prime}\right)}\left(\tilde{G}_{l}\right)$. Since the elements $\tilde{G}_{l}$ are homogeneous with respect to the variables $\xi_{1}, \ldots, \xi_{n+1}$, we can also assume $\tilde{i}_{l} \in \tilde{F}_{t-t_{l}} \mathrm{Gr}_{\bullet}^{F} D_{W^{\prime}}$, where $t_{l}=\operatorname{ord}_{(u, v)}\left(\tilde{G}_{l}\right)$. Let $i_{l} \in D_{W^{\prime}}$ be the normally ordered element which we obtain from $\tilde{i}_{l}$ by replacing $\xi_{i}$ with $\partial_{w_{i}}$. One sees easily that $i_{l} G_{l} \in F_{t} D_{W^{\prime}} \cap V^{k_{Q}} D_{W^{\prime}}$. Therefore, the element $i^{\prime}:=\sum_{l=1}^{m} i_{l} G_{l}$ has the properties

$$
\sigma_{t}\left(i^{\prime}\right)=\sigma_{t}(i)=\sigma_{t}(Q) \quad \text { and } \quad i^{\prime} \in V^{k_{Q}} D_{W^{\prime}},
$$

where the second property follows from $\operatorname{ord}_{\left(u^{\prime}, v^{\prime}\right)}\left(G_{l}\right)=\operatorname{ord}_{\left(u^{\prime}, v^{\prime}\right)}\left(\tilde{G}_{l}\right)$. We therefore have $P=$ $Q-i^{\prime}-\left(i-i^{\prime}\right)$ with $Q-i^{\prime} \in F_{t-1} D_{W^{\prime}} \cap V^{k_{Q}} D_{W^{\prime}}$. Since, obviously, we have $k \leqslant k_{Q}$, we conclude that $Q-i^{\prime} \in F_{t-1} D_{W^{\prime}} \cap V^{k} D_{W^{\prime}}$. The claim now follows by descending induction on the order $t$.

\subsection{Calculation of the Hodge filtration}

In this subsection, we want to compute the Hodge filtration on the mixed Hodge module

$$
\mathcal{H}^{0}\left(h_{A *}^{p} \mathbb{C}_{T}^{\beta, H}\right) ;
$$

recall from Section 1 that ${ }^{p} \mathbb{C}_{T}^{\beta, H}=\mathbb{C}_{T}^{\beta, H}[d] \in \operatorname{MHM}(T)$. Also recall that ${ }^{p} \mathbb{C}_{T}^{\beta, H}$ has the underlying filtered $\mathcal{D}$-module $\left(\mathcal{O}_{T}^{\beta}, F_{\bullet}^{H} \mathcal{O}_{T}^{\beta}\right)$, where the Hodge filtration is given by

$$
F_{p}^{H} \mathcal{O}_{T}^{\beta}= \begin{cases}\mathcal{O}_{T}^{\beta} & \text { for } p \geqslant 0, \\ 0 & \text { else }\end{cases}
$$

We will use several different presentations of $\mathcal{O}_{T}^{\beta}$ as a $\mathcal{D}_{T}$-module; namely, for each $\alpha=$ $\left(\alpha_{k}\right)_{k=1, \ldots, d} \in \mathbb{Z}^{d}$, we have a $D_{T}$-linear isomorphism

$$
\Gamma\left(T, \mathcal{O}_{T}^{\beta}\right) \simeq D_{T} /\left(\partial_{t_{k}} t_{k}+\beta_{k}+\alpha_{k}\right)_{k=1, \ldots, d}
$$

such that the Hodge filtration is simply the order filtration on the right-hand side.

As we have seen in Lemma 4.1, the morphism $h_{A}$ can be decomposed into the closed embedding $k_{A}: T \rightarrow W^{*}=W \backslash D$ and the canonical open embedding $l_{A}: W^{*} \rightarrow W$. We have to determine the Hodge filtration on the direct image modules for both mappings. The former is (after some coordinate change) a rather direct calculation and will be carried out in Lemma 4.14. However, understanding the behavior of the Hodge filtration under the direct image of an open embedding of the complement of a divisor (like the map $l_{A}$ ) is more subtle and at the heart of the theory of mixed Hodge modules (see, for example, [Sai90, Section (2.b)]). More precisely, since 


\section{T. Reichelt And C. Sevenheck}

the steps of the Hodge filtration of a mixed Hodge module are coherent modules over the structure sheaf of the underlying variety, the usual direct image functors are not suitable for the case of an open embedding as they do not preserve coherence. In order to circumvent this difficulty, one uses the canonical $V$-filtration along the boundary divisor, as computed in Subsection 4.1. Let us give an overview of the strategy to be used below. The actual calculation will be finished only in Theorem 4.17, the main step being Proposition 4.16.

We will need the following formula (copied from [Sai93, Proposition 4.2]), which describes the extension of a mixed Hodge module over a smooth hypersurface. Let $X$ be a smooth variety, let $t, x_{1}, \ldots x_{n}$ be local coordinates on $X$ and $j: Y \hookrightarrow X$ be a smooth hypersurface given by $t=0$. Let ${ }^{H} \mathcal{M}$ be a mixed Hodge module on $X \backslash Y$ with underlying filtered $\mathcal{D}$-module $\left(\mathcal{M}, F_{\bullet}^{H} \mathcal{M}\right)$; then

$$
F_{p}^{H} \mathcal{H}^{0} j_{+} \mathcal{M}=\sum_{i \geqslant 0} \partial_{t}^{i} F_{p-i}^{H} V^{0} \mathcal{H}^{0} j_{+} \mathcal{M}, \quad \text { where } F_{q}^{H} V^{0} \mathcal{H}^{0} j_{+} \mathcal{M}:=V^{0} \mathcal{H}^{0} j_{+} \mathcal{M} \cap j_{*}\left(F_{q}^{H} \mathcal{M}\right)
$$

with $V^{0} \mathcal{H}^{0} j_{+} \mathcal{M}$ the canonical $V$-filtration on the $\mathcal{D}$-module $\mathcal{H}^{0} j_{+} \mathcal{M} \simeq j_{*} \mathcal{M}$, as introduced in Definition 4.2.

If $Y$ is a non-smooth hypersurface locally given by $g=0$, we consider (locally) the graph embedding

$$
i_{g}: X \longrightarrow X \times \mathbb{C}_{t}, \quad x \longmapsto(x, g(x))
$$

together with its restriction $i_{g}^{\circ}: X \backslash Y \rightarrow X \times \mathbb{C}_{t}^{*}$. Notice that $i_{g}^{\circ}$ is a closed embedding. Given a mixed Hodge module $\mathcal{M}$ on $X \backslash Y$, we proceed as follows. We first extend the Hodge filtration of $\left(i_{g}^{\circ}\right)_{+} \mathcal{M}$ over the smooth divisor given by $\{t=0\}$ as explained above. Afterward, we restrict the mixed Hodge module which we obtained to the smooth divisor given by $\{t=g\}$.

After these general remarks, we come back to the situation of the torus embedding $h_{A}: T \rightarrow W$ described at the beginning of this section. Consider the commutative diagram

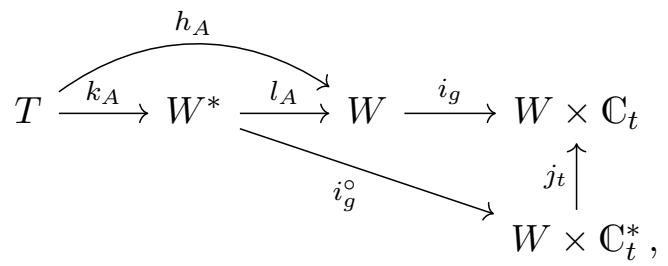

where $W^{*}:=W \backslash D=W \backslash\left\{w_{1} \cdot \ldots \cdot w_{n}=0\right\} \simeq\left(\mathbb{C}^{*}\right)^{n}$ and where $i_{g}$ is the graph embedding

$$
i_{g}: W \longrightarrow W \times \mathbb{C}_{t}, \quad w \longmapsto\left(w, w_{1} \cdot \ldots \cdot w_{n}\right)
$$

associated with the function $g: W \rightarrow \mathbb{C}_{t}, w \mapsto w_{1} \cdot \ldots \cdot w_{n}$. Notice that $i_{g} \circ l_{A}$ factors over $W \times \mathbb{C}_{t}^{*}$. We have isomorphisms

$$
i_{g+} h_{A+} \mathcal{O}_{T}^{\beta} \simeq i_{g+} l_{A+} k_{A+} \mathcal{O}_{T}^{\beta} \simeq j_{t+} i_{g+}^{\circ} k_{A+} \mathcal{O}_{T}^{\beta} .
$$

Lemma 4.14. The direct image $\mathcal{H}^{0} k_{A+} \mathcal{O}_{T}^{\beta}$ is isomorphic to the cyclic $\mathcal{D}_{W^{*}-\text { module }}$

$$
{ }^{*} \check{\mathcal{M}}_{A^{\prime}}^{\beta}:=\mathcal{D}_{W^{*}} / \check{\mathcal{I}}_{\beta}^{*},
$$

where $\check{\mathcal{I}}_{\beta}^{*}$ is the left ideal generated by $\left(\check{E}_{k}+\beta_{k}\right)_{k=1, \ldots, d}$ for $\beta=\left(\beta_{k}\right)_{k=1, \ldots, d} \in \mathbb{R}^{d}$ and $\left(\check{\square}_{\underline{m}}\right)_{\underline{m} \in \mathbb{L}_{A}}$. Furthermore, the Hodge filtration on $\check{\mathcal{M}}_{A^{\prime}}$ is equal to the induced order filtration, shifted by $n-d$; that is,

$$
F_{p}^{H *} \check{\mathcal{M}}_{A^{\prime}}=F_{p-(n-d)}^{\mathrm{ord}} \mathcal{D}_{W^{*}} / \check{\mathcal{I}}_{\beta}^{*}
$$




\section{Hypergeometric Hodge modules}

Proof. We factor the map $k_{A}$ from above in the following way. Let $A=C \cdot E \cdot F$ be the Smith normal form of $A$; that is, $C=\left(c_{p q}\right) \in \mathrm{GL}(d, \mathbb{Z}), F=\left(f_{u v}\right) \in \operatorname{GL}(n, \mathbb{Z})$ and $E=\left(I_{d}, 0_{d, n-d}\right)$. This gives rise to the maps

$$
\begin{aligned}
& k_{C}: T \longrightarrow T, \quad\left(t_{1}, \ldots, t_{d}\right) \longmapsto\left(\tilde{t}_{1}, \ldots, \tilde{t}_{d}\right)=\left(\underline{t}^{\underline{c}} \underline{c}_{1}, \ldots, \underline{t}^{\underline{c}}\right) \\
& k_{E}: T \longrightarrow\left(\mathbb{C}^{*}\right)^{n}, \quad\left(\tilde{t}_{1}, \ldots, \tilde{t}_{d}\right) \longmapsto\left(\tilde{w}_{1}, \ldots, \tilde{w}_{n}\right)=\left(\tilde{t}_{1}, \ldots, \tilde{t}_{d}, 1, \ldots, 1\right), \\
& k_{F}:\left(\mathbb{C}^{*}\right)^{n} \longrightarrow W^{*}, \quad\left(\tilde{w}_{1}, \ldots, \tilde{w}_{n}\right) \longmapsto\left(w_{1}, \ldots, w_{n}\right)=\left(\underline{\tilde{w}}^{f}, \ldots, \underline{\tilde{w}}^{f}{ }^{n}\right) .
\end{aligned}
$$

For $\gamma \in \mathbb{Z}^{d}$, we have

$$
k_{A+} \mathcal{O}_{T}^{\gamma} \simeq\left(k_{F} \circ k_{E} \circ k_{C}\right)_{+} \mathcal{O}_{T}^{\gamma} \simeq k_{F+} k_{E+} k_{C+} \mathcal{O}_{T}^{\gamma} .
$$

Since all maps and spaces involved are affine, we will work at the level of global sections. We have $\Gamma\left(T, \mathcal{O}_{T}^{\gamma}\right)=D_{T} /\left(\partial_{t_{k}} t_{k}+\gamma_{k}\right)_{k=1, \ldots, d}=D_{T} /\left(t_{k} \partial_{t_{k}}+\gamma_{k}+1\right)_{k=1, \ldots, d}$. Notice that the Hodge filtration in this presentation is simply the order filtration. Since $k_{C}$ is a change of coordinates, we have $\Gamma\left(T, \mathcal{H}^{0}\left(k_{C}\right)_{+} \mathcal{O}_{T}^{\gamma}\right) \simeq D_{T} /\left(\sum_{i=1}^{d} c_{k i} \tilde{t}_{i} \partial_{\tilde{t}_{i}}+\gamma_{k}+1\right)_{k=1, \ldots, d}$, and again the Hodge filtration is equal to the order filtration. We now calculate $\Gamma\left(\left(\mathbb{C}^{*}\right)^{n}, k_{E+} k_{C+} \mathcal{O}_{T}^{\gamma}\right)$. We have

$$
\begin{aligned}
\Gamma\left(\left(\mathbb{C}^{*}\right)^{n}, \mathcal{H}^{0} k_{E+} k_{C+} \mathcal{O}_{T}^{\gamma}\right) & \simeq \Gamma\left(T, \mathcal{H}^{0} k_{C+} \mathcal{O}_{T}^{\gamma}\right)\left[\partial_{\tilde{w}_{d+1}}, \ldots, \partial_{\tilde{w}_{n}}\right] \\
& \simeq D_{\left(\mathbb{C}^{*}\right)^{n}} /\left(\left(\sum_{i=1}^{d} c_{k i} \tilde{w}_{i} \partial_{\tilde{w}_{i}}+\gamma_{k}+1\right)_{k=1, \ldots, d},\left(\tilde{w}_{i}-1\right)_{i=d+1, \ldots, n}\right) .
\end{aligned}
$$

The Hodge filtration is (cf. [Sai93, formula (1.8.6)])

$$
\begin{aligned}
F_{p+(n-d)}^{H}\left(\Gamma\left(\left(\mathbb{C}^{*}\right)^{n}, \mathcal{H}^{0} k_{E+} k_{C+} \mathcal{O}_{T}^{\gamma}\right)\right) & =\sum_{p_{1}+p_{2}=p} F_{p_{1}}^{H} \Gamma\left(T, \mathcal{H}^{0} k_{C+} \mathcal{O}_{T}^{\gamma}\right) \otimes \underline{\partial}^{p_{2}} \\
& =\sum_{p_{1}+p_{2}=p} F_{p_{1}}^{\text {ord }} \Gamma\left(T, \mathcal{H}^{0} k_{C+} \mathcal{O}_{T}^{\gamma}\right) \otimes \underline{\partial}^{p_{2}} \\
& =F_{p}^{\text {ord }}\left(\Gamma\left(\left(\mathbb{C}^{*}\right)^{n}, \mathcal{H}^{0} k_{E+} k_{C+} \mathcal{O}_{T}^{\gamma}\right)\right) .
\end{aligned}
$$

Hence, we see that the Hodge filtration on the presentation (4.12) shifted by $n-d$ is equal to the order filtration; that is, $F_{p+(n-d)}^{H}=F_{p}^{\text {ord }}$.

The map $k_{F}$ is again a change of coordinates, so we have

$$
\begin{aligned}
& \Gamma\left(\left(\mathbb{C}^{*}\right)^{n}, \mathcal{H}^{0} k_{F+} k_{E_{+}} k_{C+} \mathcal{O}_{T}^{\gamma}\right) \simeq D_{\left(\mathbb{C}^{*}\right)^{n}} /\left(\left(\sum_{j=1}^{n} a_{k j} w_{j} \partial_{w_{j}}+\gamma_{k}+1\right)_{k=1, \ldots, d},\left(\underline{w}^{\underline{m}} \underline{w}_{i}-1\right)_{i=d+1, \ldots, n}\right) \\
& \simeq D_{\left(\mathbb{C}^{*}\right)^{n}} /\left(\left(\sum_{j=1}^{n} a_{k j} w_{j} \partial_{w_{j}}+\gamma_{k}+1\right)_{k=1, \ldots, d},\left(\check{\square}_{\underline{m}}\right)_{\underline{m} \in \mathbb{L}_{A}}\right),
\end{aligned}
$$

where the $\underline{m}_{i}$ are the columns of the inverse matrix $M=F^{-1}$. The first isomorphism follows from the equality $A=C \cdot E \cdot F$. The second isomorphism follows from the fact that an element $\underline{m} \in \mathbb{Z}^{n}$ is a relation between the columns of $A$ if and only if it is a relation between the columns of $E \cdot F$. So the Hodge filtration on the presentation (4.13) shifted by $n-d$ is again the order filtration. We have

$$
\sum_{j=1}^{n} a_{k j} w_{j} \partial_{w_{j}}+\gamma_{k}+1=\sum_{j=1}^{n} a_{k j} \partial_{w_{j}} w_{j}-\sum_{j=1}^{n} a_{k j}+\gamma_{k}+1
$$




\section{T. Reichelt And C. Sevenheck}

Setting $\gamma_{k}:=\sum_{j=1}^{n} a_{k j}+\beta_{k}-1$ shows that

$$
{ }^{*} \check{\mathcal{M}}_{A^{\prime}}^{\beta}=\mathcal{H}^{0} k_{A+} \mathcal{O}_{T}^{\sum_{j=1}^{n} \underline{a}_{j}+\beta-1} \simeq \mathcal{H}^{0} k_{A+} \mathcal{O}_{T}^{\beta},
$$

where the last isomorphism is given by right multiplication with $\underline{t}^{-\sum_{j=1}^{n} \underline{a}_{j}+\mathbf{1}}$ (here $\mathbf{1}:=(1, \ldots, 1)$ $\left.\in \mathbb{Z}^{d}\right)$.

The next step is to compute the Hodge filtration of $h_{A+} \mathcal{O}_{T}^{\beta} \simeq l_{A+} k_{A+} \mathcal{O}_{T}^{\beta}$ from that of $k_{A+} \mathcal{O}_{T}^{\beta}$. As the map $l_{A}$ is an open embedding of the complement of a normal crossing divisor, we need to consider the graph embedding $i_{g}^{\circ}$ with respect to the function $g=w_{1} \cdot \ldots \cdot w_{n}$. We proceed as described at the beginning of this subsection; that is, we first extend the module $i_{g+}^{\circ} k_{A+} \mathcal{O}_{T}^{\beta}$ over the smooth divisor $\{t=0\}$.

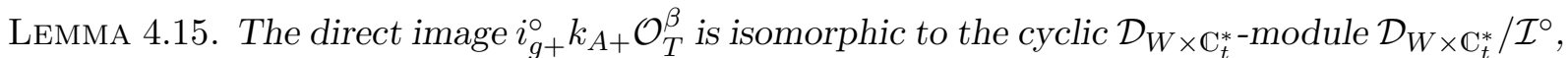
where $\mathcal{I}^{\circ}$ is the left ideal generated by $\left(\check{E}_{k}^{\prime}+\beta_{k}\right)_{k=1, \ldots, d}$ for $\beta=\left(\beta_{k}\right)_{k=1, \ldots, d} \in \mathbb{R}^{d}$ and $\left(\check{\square}_{\underline{m}}\right)_{\underline{m} \in \mathbb{L}_{A^{\prime}}}$. Recall that the vector fields $\check{E}_{k}^{\prime}$ have been defined in formula (4.2) as $\check{E}_{k}^{\prime}:=\sum_{i=1}^{n} b_{k i} \partial_{w_{i}} w_{i}+c_{k} \partial_{t} t$ for $k=1, \ldots, d$. Furthermore, the Hodge filtration shifted by $n-d+1$ is equal to the induced order filtration; that is, we have

$$
F_{p}^{H} i_{g+}^{\circ} k_{A+} \mathcal{O}_{T}^{\beta} \simeq F_{p-(n-d+1)}^{\text {ord }} \mathcal{D}_{W \times \mathbb{C}_{t}^{*}} / \mathcal{I}^{\circ}
$$

Proof. We define

$$
\widetilde{W}:=\left(W^{*} \times \mathbb{C}_{\tilde{t}}\right) \backslash\{\tilde{t}+g(\underline{w})=0\}
$$

and factor the map $i_{g}^{\circ}$ in the following way. Set

$$
l_{1}: W^{*} \longrightarrow \widetilde{W}, \quad \underline{w} \longmapsto(\underline{w}, 0) \quad \text { and } \quad l_{2}: \widetilde{W} \longrightarrow W^{*} \times \mathbb{C}_{t}^{*}, \quad(\underline{w}, \tilde{t}) \longmapsto(\underline{w}, \tilde{t}+g(\underline{w})),
$$

and let $l_{3}: W^{*} \times \mathbb{C}_{t}^{*} \rightarrow W \times \mathbb{C}_{t}^{*}$ be the canonical inclusion. We have $i_{g}^{\circ}=l_{3} \circ l_{2} \circ l_{1}$. For the convenience of the reader, let us summarize these maps in the following diagram:

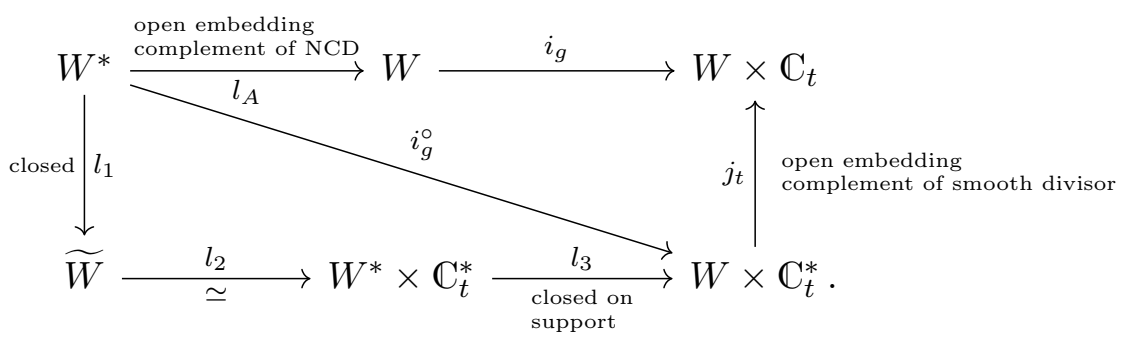

Notice again that all spaces involved are affine; hence, we will work with the modules of global sections. Since $l_{1}$ is just the inclusion of a coordinate hyperplane, we have

$$
\Gamma\left(\widetilde{W}, \mathcal{H}^{0} l_{1+} k_{A+} \mathcal{O}_{T}^{\beta}\right) \simeq \Gamma\left(W^{*}, \mathcal{H}^{0} k_{A+} \mathcal{O}_{T}^{\beta}\right)\left[\partial_{\tilde{t}}\right] .
$$

The Hodge filtration is given by

$$
\Gamma\left(\widetilde{W}, F_{p+1}^{H}\left(\mathcal{H}^{0} l_{1+} k_{A+} \mathcal{O}_{T}^{\beta}\right)\right) \simeq \sum_{p_{1}+p_{2}=p} \Gamma\left(W^{*}, F_{p_{1}}^{H} \mathcal{H}^{0} k_{A+} \mathcal{O}_{T}^{\beta}\right) \otimes \partial_{\tilde{t}}^{p_{2}} .
$$

Notice that $\Gamma\left(\widetilde{W}, \mathcal{H}^{0} l_{1+} k_{A+} \mathcal{O}_{T}^{\beta}\right) \simeq D_{\widetilde{W}} / I_{1}^{\circ}$, where $I_{1}^{\circ}$ is the left ideal generated by $\left(\check{E}_{k}+\beta_{k}\right)_{k=1, \ldots, d}$, $\left(\check{\square}_{\underline{m}}\right)_{\underline{m} \in \mathbb{L}_{A}}$ and $\tilde{t}$.

Under this isomorphism, the Hodge filtration on $\Gamma\left(\widetilde{W}, \mathcal{H}^{0} l_{1+} k_{A+} \mathcal{O}_{T}^{\beta}\right)$ shifted by $(n-d)+1$ is equal to the order filtration, by Lemma 4.14 and (4.15). The map $l_{2}$ is just a change of coordinates; 


\section{Hypergeometric Hodge modules}

hence, under the substitutions $\tilde{t} \mapsto t=\tilde{t}+g(\underline{w})$ and $w_{i} \partial_{w_{i}} \mapsto w_{i} \partial_{w_{i}}+g(\underline{w}) \partial_{t} \equiv w_{i} \partial_{w_{i}}+\partial_{t} t$ for $i=1, \ldots, n$ and by using the presentation of $\mathcal{H}^{0}\left(k_{A+} \mathcal{O}_{T}^{\beta}\right)$ as acyclic $\mathcal{D}$-module, we get that

$$
\begin{aligned}
\Gamma\left(W^{*} \times \mathbb{C}_{t}^{*}, \mathcal{H}^{0} l_{2+} l_{1+} k_{A+} \mathcal{O}_{T}^{\beta}\right) & \simeq D_{W^{*} \times \mathbb{C}_{t}^{*}} /\left(\left(\check{E}_{k}^{\prime}+\beta_{k}\right)_{k=1, \ldots, d}+\left(\check{\square}_{\underline{m}}\right)_{\underline{m} \in \mathbb{L}_{A}}+\left(t-w_{1} \cdot \ldots \cdot w_{n}\right)\right) \\
& \simeq D_{W^{*} \times \mathbb{C}_{t}^{*}} /\left(\left(\check{E}_{k}^{\prime}+\beta_{k}\right)_{k=1, \ldots, d}+\left(\check{\square}_{\underline{m}}\right)_{\underline{m} \in \mathbb{L}_{A^{\prime}}}\right),
\end{aligned}
$$

where $\check{E}_{k}^{\prime}$ was defined in formula (4.2) and $A^{\prime}$ is the matrix defined just before that formula. Notice that the Hodge filtration shifted by $(n-d)+1$ is again equal to the order filtration.

Since the support of $\mathcal{H}^{0} l_{2+} l_{1+} k_{A+} \mathcal{O}_{T}^{\beta}$ lies in the subvariety $\{t=g(\underline{w})\}$, the closure of the support in $W \times \mathbb{C}_{t}^{*}$ does not meet $D \times \mathbb{C}_{t}^{*}$. We conclude that

$$
\begin{aligned}
\Gamma\left(W \times \mathbb{C}_{t}^{*}, \mathcal{H}^{0} i_{g+}^{\circ} k_{A+} \mathcal{O}_{T}^{\beta}\right) & \simeq \Gamma\left(W \times \mathbb{C}_{t}^{*}, \mathcal{H}^{0} l_{3+} l_{2+} l_{1+} k_{A+} \mathcal{O}_{T}^{\beta}\right) \\
& \simeq \Gamma\left(W^{*} \times \mathbb{C}_{t}^{*}, \mathcal{H}^{0} l_{2+} l_{1+} k_{A+} \mathcal{O}_{T}^{\beta}\right) \\
& \simeq D_{W^{*} \times \mathbb{C}_{t}^{*}} /\left(\left(\check{E}_{k}^{\prime}+\beta_{k}\right)_{k=1, \ldots, d}+\left(\check{\square}_{\underline{m}}\right)_{\underline{m} \in \mathbb{L}_{A^{\prime}}}\right) \\
& \simeq D_{W \times \mathbb{C}_{t}^{*}} /\left(\left(E_{k}^{\prime}+\beta_{k}\right)_{k=1, \ldots, d}+\left(\check{\square}_{\underline{m}}\right)_{\underline{m} \in \mathbb{L}_{A^{\prime}}}\right) .
\end{aligned}
$$

The Hodge filtration is then simply extended by using the formula

$$
F_{p}^{H} \mathcal{H}^{0} i_{g+}^{\circ} k_{A+} \mathcal{O}_{T}^{\beta} \simeq F_{p}^{H} \mathcal{H}^{0} l_{3+} l_{2+} l_{1+} k_{A+} \mathcal{O}_{T}^{\beta} \simeq l_{3 *} F_{p}^{H} \mathcal{H}^{0} l_{2+} l_{1+} k_{A+} \mathcal{O}_{T}^{\beta} .
$$

Proposition 4.16. Let $\beta \in \mathbb{R}^{d} \backslash \operatorname{sRes}(A)$. The direct image $\mathcal{H}^{0} j_{t+} i_{g+}^{\circ} k_{A+} \mathcal{O}_{T}^{\beta}$ is isomorphic to the quotient $\check{\mathcal{M}}_{A^{\prime}}^{\beta}=\mathcal{D}_{W \times \mathbb{C}_{t}} / \mathcal{I}^{\prime}$, where $\mathcal{I}^{\prime}$ is the left ideal generated by $\left(\check{E}_{k}^{\prime}+\beta_{k}\right)_{k=1, \ldots, d}$ and $\left(\check{\square}_{\underline{m}}\right)_{\underline{m} \in \mathbb{L}_{A^{\prime}}}$. Furthermore, if $\beta \in \mathfrak{A}_{A}$, then the Hodge filtration on $\mathcal{D}_{W \times \mathbb{C}_{t}} / \mathcal{I}^{\prime}$ shifted by $(n-d)+1$ is equal to the induced order filtration; that is,

$$
F_{p}^{H} \mathcal{H}^{0} j_{t+} i_{g+}^{\circ} k_{A+} \mathcal{O}_{T}^{\beta}=F_{p-(n-d+1)}^{\text {ord }} \mathcal{D}_{W \times \mathbb{C}_{t}} / \mathcal{I}^{\prime}
$$

Proof. First recall that we have an isomorphism $\mathcal{H}^{0} j_{t+} i_{g+}^{\circ} k_{A+} \mathcal{O}_{T}^{\beta} \simeq \mathcal{H}^{0} i_{g+} h_{A+} \mathcal{O}_{T}^{\beta}$. The composed map $i_{g} \circ h_{A}$ is a torus embedding given by the matrix $A^{\prime}$. Hence, we have an isomorphism $\mathcal{H}^{0} j_{t+} i_{g+}^{\circ} k_{A+} \mathcal{O}_{T}^{\beta} \simeq \check{\mathcal{M}}_{A^{\prime}}^{\beta}$ for $\beta \in \mathbb{R}^{d}$ and $\beta \notin \operatorname{sRes}\left(A^{\prime}\right)=\operatorname{sRes}(A)$. This shows the first claim.

For the second statement, suppose $\beta \in \mathfrak{A}_{A}$. The formula for extending the Hodge filtration over the smooth divisor $\{t=0\}$ is

$$
F_{p}^{H} \check{\mathcal{M}}_{A^{\prime}}^{\beta}=\sum_{i \geqslant 0} \partial_{t}^{i}\left(V^{0} \check{\mathcal{M}}_{A^{\prime}}^{\beta} \cap j_{t *} j_{t}^{-1} F_{p-i}^{H} \check{\mathcal{M}}_{A^{\prime}}^{\beta}\right)
$$

At the level of global sections, the adjunction morphism $\check{\mathcal{M}}_{A^{\prime}}^{\beta} \longrightarrow j_{t+} j_{t}^{+} \check{\mathcal{M}}_{A^{\prime}}^{\beta}$ is given by the

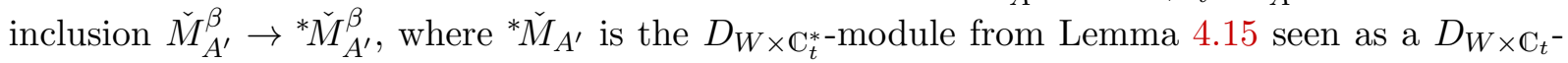
module. Hence, at the level of global sections, formula (4.17) becomes

$$
F_{p}^{H} \check{M}_{A^{\prime}}^{\beta}=\sum_{i \geqslant 0} \partial_{t}^{i}\left(V^{0} \check{M}_{A^{\prime}}^{\beta} \cap F_{p-i}^{H}{ }^{*} \check{M}_{A^{\prime}}^{\beta}\right) \text {. }
$$

Since we have $F_{p+(n-d+1)}^{H} \check{M}_{A^{\prime}}^{\beta}=F_{p}^{\text {ord } *} \check{M}_{A^{\prime}}^{\beta}$ by the same lemma, we conclude that $F_{n-d^{H}}^{H} \check{M}_{A^{\prime}}^{\beta}=0$. The element $1 \in \check{M}_{A^{\prime}}^{\beta}$ is in $V^{0} \check{M}_{A^{\prime}}^{\beta}$ by Proposition 4.8 and $1 \in F_{(n-d)+1}^{H}{ }^{*} \check{M}_{A^{\prime}}^{\beta}=F_{0}^{\text {ord } *} \check{M}_{A^{\prime}}^{\beta}$ and therefore $1 \in F_{(n-d)+1}^{H} \check{M}_{A^{\prime}}^{\beta}$. Notice that both $\left(\check{M}_{A^{\prime}}^{\beta}, F_{\bullet}^{H}\right)$ and $\left(\check{M}_{A^{\prime}}^{\beta}, F_{\bullet}^{\text {ord }}\right)$ are cyclic, well-filtered

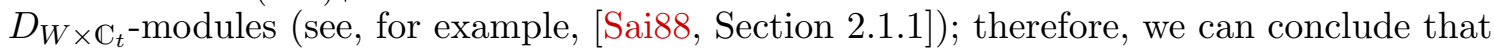

$$
F_{p}^{\mathrm{ord}} \check{M}_{A^{\prime}}^{\beta} \subset F_{p+(n-d+1)}^{H} \check{M}_{A^{\prime}}^{\beta} .
$$




\section{T. Reichelt and C. Sevenheck}

In order to show the reverse inclusion, we have to show

$$
F_{p}^{\text {ord }} \check{M}_{A^{\prime}}^{\beta} \supset F_{p+(n-d+1)}^{H} \check{M}_{A^{\prime}}^{\beta}=\sum_{i \geqslant 0} \partial_{t}^{i}\left(V^{0} \check{M}_{A^{\prime}}^{\beta} \cap F_{p+(n-d+1)-i}^{H} \check{M}_{A^{\prime}}^{\beta}\right)=\sum_{i \geqslant 0} \partial_{t}^{i}\left(V_{\text {ind }}^{0} \check{M}_{A^{\prime}}^{\beta} \cap F_{p-i}^{\text {ord } *} \check{M}_{A^{\prime}}^{\beta}\right)
$$

for all $p \geqslant 0$, where the last equality follows from Proposition 4.8 and Lemma 4.15. Since we have

$$
F_{p}^{\text {ord }} \check{M}_{A^{\prime}}^{\beta} \supset \partial_{t}^{i} F_{p-i}^{\text {ord }} \check{M}_{A^{\prime}}^{\beta} \quad \text { for } p \geqslant 0 \text { and } 0 \leqslant i \leqslant p
$$

it remains to show

$$
F_{p-i}^{\mathrm{ord}} \check{M}_{A^{\prime}}^{\beta} \supset V_{\text {ind }}^{0} \check{M}_{A^{\prime}}^{\beta} \cap F_{p-i}^{\mathrm{ord} *} \check{M}_{A^{\prime}}^{\beta} \text { for } p \geqslant 0 \text { and } 0 \leqslant i \leqslant p
$$

and

$$
F_{p}^{\text {ord }} \check{M}_{A^{\prime}}^{\beta} \supset V_{\text {ind }}^{0} \check{M}_{A^{\prime}}^{\beta} \cap F_{p}^{\text {ord } *} \check{M}_{A^{\prime}}^{\beta} \quad \text { for } p \geqslant 0 .
$$

Now, let $[P] \in V_{\text {ind }}^{0} \check{M}_{A^{\prime}}^{\beta} \cap F_{p}^{\text {ord } *} \check{M}_{A^{\prime}}^{\beta}$; then $P \in D_{W \times \mathbb{C}_{t}}$ can be written as

$$
P=t^{-k} P_{k}+t^{-k+1} P_{k-1}+\cdots
$$

with $P_{i} \in \mathbb{C}\left[w_{1}, \ldots, w_{n}\right]\left\langle\partial_{t}, \partial_{w_{1}}, \ldots, \partial_{w_{n}}\right\rangle$ and $P_{k} \neq 0$. Since $t^{k} \cdot[P] \in V_{\text {ind }}^{k} \check{M}_{A^{\prime}}^{\beta} \cap F_{p}^{\text {ord }} \check{M}_{A^{\prime}}^{\beta}$ it is enough to prove

$$
t^{k} F_{p}^{\text {ord }} \check{M}_{A^{\prime}}^{\beta} \supset V_{\text {ind }}^{k} \check{M}_{A^{\prime}}^{\beta} \cap F_{p}^{\text {ord }} \check{M}_{A^{\prime}}^{\beta} \quad \text { for } \quad p \geqslant 0 .
$$

Given an element $[Q] \in V_{\text {ind }}^{k} \check{M}_{A^{\prime}}^{\beta} \cap F_{p}^{\text {ord }} \check{M}_{A^{\prime}}^{\beta}$, using Proposition 4.9, we can find a $Q^{\prime} \in V^{k} D_{W \times \mathbb{C}_{t}} \cap$ $F_{p} D_{W \times \mathbb{C}_{t}}$ with $[Q]=\left[Q^{\prime}\right]$. But this element $Q^{\prime}$ can be written as a linear combination of monomials $t^{l_{0}} w_{1}^{l_{1}} \cdots w_{n}^{l_{n}} \partial_{t}^{p_{0}} \partial_{w_{1}}^{p_{1}} \cdots \partial_{w_{n}}^{p_{n}}$ with $p_{0}+\cdots+p_{n} \leqslant p$ and $l_{0}-p_{0} \geqslant k$; hence, $\left[Q^{\prime}\right] \in t^{k} F_{p}^{\text {ord }} \check{M}_{A^{\prime}}^{\beta}$. This shows the statement in the case $\mathbb{N} A \neq \mathbb{Z}^{d}$ (recall that $\mathbb{N} A \neq \mathbb{Z}^{d}$ was a condition used in Proposition 4.9).

In the case $\mathbb{N} A=\mathbb{Z}^{d}$, the support of $\check{\mathcal{M}}_{A^{\prime}}^{\beta}$ is disjoint from the divisor $\{t=0\}$; hence, the extension of the Hodge filtration is simply given by $F_{p}^{H} \check{\mathcal{M}}_{A^{\prime}}^{\beta}=j_{t *} F_{p}^{H *} \check{\mathcal{M}}_{A^{\prime}}^{\beta}$. Since $j_{t}$ is an open embedding, we have $\check{M}_{A^{\prime}}^{\beta}={ }^{*} \check{M}_{A^{\prime}}^{\beta}$ and therefore also $F_{p}^{H} \check{M}_{A^{\prime}}^{\beta}=F_{p}^{H *} \check{M}_{A^{\prime}}^{\beta}$. This shows the claim.

Now, we want to deduce the Hodge filtration on $h_{A+} \mathcal{O}_{T}^{\beta}$ from Proposition 4.16.

Theorem 4.17. Let $\mathbb{N} A=\mathbb{Z}^{d} \cap \mathbb{R}_{\geqslant 0} A$ and $\beta \in \mathbb{R}^{d} \backslash \operatorname{sRes}(A)$. The direct image $h_{A+} \mathcal{O}_{T}^{\beta}$ is isomorphic to the cyclic $D_{W}$-module $\check{\mathcal{M}}_{A}^{\beta}:=\mathcal{D}_{W} / \check{\mathcal{I}}$, where $\check{\mathcal{I}}$ is the left ideal generated by $\left(\check{E}_{k}+\beta_{k}\right)_{k=1, \ldots, d}$ and $\left(\check{\square}_{\underline{m}}\right)_{\underline{m} \in \mathbb{L}_{A}}$. For $\beta \in \mathfrak{A}_{A}$, the Hodge filtration on $\check{\mathcal{M}}_{A}^{\beta}$ is equal to the order filtration shifted by $n-d$; that is,

$$
F_{p+(n-d)}^{H} \check{\mathcal{M}}_{A}^{\beta}=F_{p}^{\text {ord }} \check{\mathcal{M}}_{A}^{\beta} .
$$

Proof. Recall that we have $j_{t} \circ i_{g}^{\circ} \circ k_{A}=i_{g} \circ h_{A}$, where $i_{f}$ is the graph embedding from (4.11). The map $i_{f}$ can be factored as $l_{g} \circ i_{o}$, where

$$
i_{0}: W \longrightarrow W \times \mathbb{C}_{\widetilde{t}}, \quad \underline{w} \longmapsto(\underline{w}, 0) \quad \text { and } \quad l_{g}: W \times \mathbb{C}_{\widetilde{t}} \longrightarrow W \times \mathbb{C}_{t}, \quad(\underline{w}, \widetilde{t}) \longmapsto(\underline{w}, \tilde{t}+g(\underline{w})) .
$$




\section{HypergeOMetric Hodge MOdules}

Once again, we summarize the relevant maps in the following diagram:

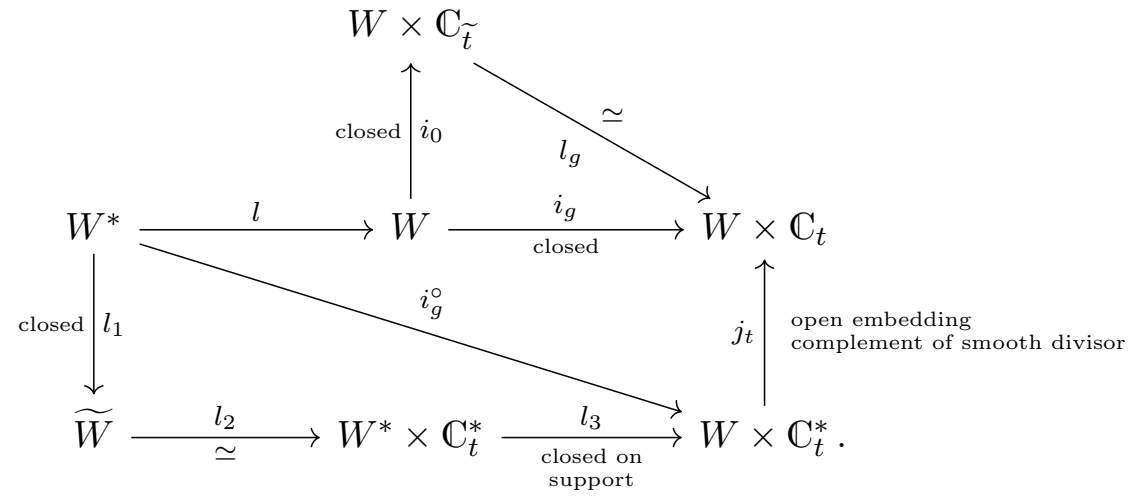

We first compute $\mathcal{H}^{0}\left(l_{g}^{-1}\right)_{+} \check{\mathcal{M}}_{A^{\prime}}^{\beta}$ with its corresponding Hodge filtration. Since $\left(l_{g}\right)^{-1}$ is just a coordinate change, similarly to formula (4.16), we get

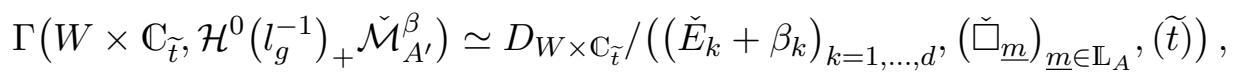

where the Hodge filtration on the right-hand side is the induced order filtration shifted by $n-d+1$. Notice that the right-hand side of (4.18) is simply $\check{M}_{A}^{\beta}\left[\partial_{\bar{t}}\right]$; hence, the Hodge filtration on

$$
\check{M}_{A}^{\beta}=\Gamma\left(W, \check{\mathcal{M}}_{A}^{\beta}\right)=\Gamma\left(W \times \mathbb{C}_{\widetilde{t}}, \mathcal{H}^{0} i_{0}^{+}\left(l_{g}^{-1}\right)_{+} \check{\mathcal{M}}_{A^{\prime}}^{\beta}\right)
$$

is simply the order filtration shifted by $n-d$ by [Sai88, Proposition 3.2.2(iii)].

Remark 4.18. Let $\mathcal{O}_{\mathbb{C}^{*}}^{\beta}=\mathcal{D}_{\mathbb{C}^{*}} /\left(\partial_{t} t+\beta\right)$ and $j_{0}: \mathbb{C}^{*} \longrightarrow \mathbb{C}$ be the inclusion. If $\beta \notin\{-1,-2,-3, \ldots\}$, then

$$
j_{0+} \mathcal{O}_{\mathbb{C}^{*}}^{\beta} \simeq \mathcal{D}_{\mathbb{C}} /\left(\partial_{t} t+\beta\right)
$$

as well as

$$
j_{0 \dagger} \mathcal{O}_{\mathbb{C}^{*}}^{-\beta-1} \simeq \mathbb{D} j_{0+} \mathbb{D} \mathcal{O}_{\mathbb{C}^{*}}^{\beta} \simeq \mathbb{D} j_{0+} \mathcal{D}_{\mathbb{C}^{*}} /\left(\partial_{t} t+\beta\right) \simeq \mathbb{D} \mathcal{D}_{\mathbb{C}} /\left(\partial_{t} t+\beta\right) \simeq \mathcal{D}_{\mathbb{C}} /\left(t \partial_{t}-\beta\right) .
$$

If, additionally, $\beta \in(-1,0]$, then by Theorem 4.17,

$$
\left(j_{0+} \mathcal{O}_{\mathbb{C}^{*}}^{\beta}, F_{\bullet}^{H}\right) \simeq\left(\mathcal{D}_{\mathbb{C}} /\left(\partial_{t} t+\beta\right), F_{\bullet}^{\text {ord }}\right) .
$$

In order to compute the Hodge filtration on $j_{0 \dagger} \mathcal{O}_{\mathbb{C}^{*}}^{-\beta-1}$, we use that $\left(\mathbb{D}_{\mathbb{C}^{*}}^{-\beta-1}, F_{p}^{H}\right)=\left(\mathcal{D}_{\mathbb{C}^{*}} /\left(\partial_{t} t+\right.\right.$ $\left.\beta), F_{\bullet-1}^{\text {ord }}\right)$ and therefore $\left(j_{0+} \mathbb{D O}_{\mathbb{C}^{*}}^{-\beta-1}, F_{p}^{H}\right)=\left(\mathcal{D}_{\mathbb{C}} /\left(\partial_{t} t+\beta\right), F_{\bullet-1}^{\text {ord }}\right)$, since we assumed $\beta \in(-1,0]$. We use the filtered resolution

$$
\left(\mathcal{D}_{\mathbb{C}^{*}}, F_{\bullet-2}^{\text {ord }}\right) \stackrel{\partial_{t} t+\beta}{\longrightarrow}\left(\mathcal{D}_{\mathbb{C}^{*}}, F_{\bullet-1}^{\text {ord }}\right)
$$

to resolve $\left(\mathcal{D}_{\mathbb{C}} /\left(\partial_{t} t+\beta\right), F_{\bullet-1}^{\text {ord }}\right)$ and apply $\mathcal{H o m}\left(-,\left(\mathcal{D}_{\mathbb{C}}, F_{\bullet-2}^{\text {ord }}\right) \otimes \omega_{X}^{\vee}\right)$ to compute the dual of $\left(\mathcal{D}_{\mathbb{C}} /\left(\partial_{t} t+\beta\right), F_{\bullet-1}^{\text {ord }}\right)$ (cf. [Sai94, p. 55] for the choice of filtration on $\left.\mathcal{D}_{\mathbb{C}} \otimes \omega_{X}^{\vee}\right)$. This gives

$$
\left(j_{0 \dagger} \mathcal{O}_{\mathbb{C}^{*}}^{-\beta-1}, F_{p}^{H}\right) \simeq\left(\mathcal{D}_{\mathbb{C}} /\left(t \partial_{t}-\beta\right), F_{p}^{\text {ord }}\right)
$$

for $\beta \in(-1,0]$.

\section{Integral transforms of torus embeddings}

In this section, we investigate how the Hodge filtration of $\mathbb{C}_{T}^{H, \beta}$ behaves under a certain integral transformation which depends on a matrix $\widetilde{A}$ and a parameter $\beta_{0}$. We show that the outcome 


\section{T. Reichelt And C. Sevenheck}

of this transform is isomorphic to the GKZ system $\mathcal{M}_{\widetilde{A}}^{\left(\beta_{0}, \beta\right)}$ (cf. Proposition 5.4). In the special case $\beta_{0} \in \mathbb{Z}$, we will show that the integral transformation mentioned above is isomorphic to the Radon transform of a torus embedding.

The Radon transformation has been extensively used in the previous papers [Rei14, RS17] in order to study hyperplane sections of toric varieties or, more precisely, fibers of Laurent polynomials and their compactifications.

In the first subsection below, we give a brief reminder of how certain GKZ systems can be constructed using the Radon transformation. The second subsection introduces an integral transform that is able to produce all GKZ systems $\mathcal{M}_{\widetilde{A}}^{\widetilde{\beta}}$ with $\widetilde{\beta} \notin \operatorname{ses}(\widetilde{A})$ and homogeneous $\widetilde{A}$.

The next subsections (until Subsection 5.9) constitute the main part of this section, where we study in detail how the various functors entering in the definition of this integral transformation act on the twisted structure sheaf. One can roughly divide the construction in two parts: In Subsection 5.3, we calculate the push-forward of a tensor product between the twisted structure sheaf and a kernel to a partial compactification. Then one has to study the projection to the parameter space (that is, the space on which the GKZ system is defined). The calculation of the behavior of the filtration steps is non-trivial, as the higher direct images of these filtration steps, being coherent $\mathcal{O}$-modules, do not, a priori, vanish. However, we can show that this is actually the case in the current situation. We formulate this result in the language of $\mathscr{R}$-modules (that is, using the Rees construction for filtered $\mathcal{D}$-modules) and make extensive use of (variants of) the Euler-Koszul complex of hypergeometric modules. All these intermediate steps are contained in Subsections 5.4-5.9. A very important technical result is the calculation of some local cohomology groups of a certain semigroup ring, contained in Subsection 5.8. The culminating point is then Theorem 5.35, which gives a precise description of the Hodge filtration on certain GKZ systems.

\subsection{Hypergeometric modules, Gauß-Manin systems and the Radon transformation}

Here we give a brief reminder on the relationship between GKZ hypergeometric systems and Gauß-Manin systems of families of Laurent polynomials as developed in [Rei14].

As in [RS15, RS17], we will consider a homogenization of the above systems. Namely, given the matrix $A=\left(a_{k i}\right)$, we consider the system $\mathcal{M}_{\widetilde{A}}^{\widetilde{\beta}}$, where $\widetilde{A}$ is the $(d+1) \times(n+1)$ integer matrix

$$
\widetilde{A}:=\left(\underline{\widetilde{a}}_{0}, \ldots, \underline{\widetilde{a}}_{n}\right):=\left(\begin{array}{cccc}
1 & 1 & \ldots & 1 \\
0 & a_{11} & \ldots & a_{1 n} \\
\vdots & \vdots & & \vdots \\
0 & a_{d 1} & \ldots & a_{d n}
\end{array}\right)
$$

and $\widetilde{\beta} \in \mathbb{C}^{d+1}$.

In order to show that such a homogenized GKZ system comes from geometry, we have to review briefly the so-called Radon transformation for $\mathcal{D}$-modules; this was introduced by Brylinski [Bry86], and variants were later added by D'Agnolo and Eastwood [DE03].

Let $W$ be the dual vector space of $V$ with coordinates $w_{0}, \ldots, w_{n}$, and let $\lambda_{0}, \ldots, \lambda_{n}$ be coordinates for $V$. We denote by $Z \subset \mathbb{P}(W) \times V$ the universal hyperplane given by $Z:=$ 


\section{HypergeOMetric Hodge MOdules}

$\left\{\sum_{i=0}^{n} \lambda_{i} w_{i}=0\right\}$ and denote its complement by $U:=(\mathbb{P}(W) \times V) \backslash Z$. Consider the diagram

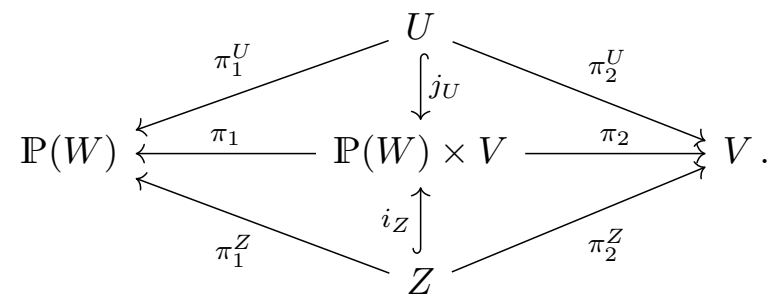

From here on, we will use several variants of the so-called Radon transformation in the derived category of mixed Hodge modules. These are functors from $D^{b} \operatorname{MHM}(\mathbb{P}(W))$ to $D^{b} \operatorname{MHM}\left(\mathcal{D}_{V}\right)$ given by

$$
\begin{aligned}
{ }^{*} \mathcal{R}(M) & :=\pi_{2 *}^{Z}\left(\pi_{1}^{Z}\right)^{*} M \simeq \pi_{2 *} i_{Z *} i_{Z}^{*} \pi_{1}^{*} M, \\
{ }^{!} \mathcal{R}(M) & :=\mathbb{D} \circ{ }^{*} \mathcal{R} \circ \mathbb{D}(M) \simeq \pi_{2 *}^{Z}\left(\pi_{1}^{Z}\right)^{!} M \simeq \pi_{2 *} i_{Z *} i_{Z}^{!} \pi_{1}^{!} M, \\
{ }^{*} \mathcal{R}_{\mathrm{cst}}(M) & :=\pi_{2 *} \pi_{1}^{*} M, \\
{ }^{!} \mathcal{R}_{\mathrm{cst}}(M) & :=\mathbb{D} \circ{ }^{*} \mathcal{R}_{\mathrm{cst}} \circ \mathbb{D}(M) \simeq \pi_{2 *} \pi_{1}^{!} M, \\
{ }^{*} \mathcal{R}_{c}^{\circ}(M) & :=\pi_{2 !}^{U}\left(\pi_{1}^{U}\right)^{*}(M) \simeq \pi_{2 *} j_{U !} j_{U}^{*} \pi_{1}^{*}(M), \\
{ }^{!} \mathcal{R}^{\circ}(M) & :=\mathbb{D} \circ{ }^{*} \mathcal{R}_{c}^{\circ} \circ \mathbb{D}(M) \simeq \pi_{2 *}^{U}\left(\pi_{1}^{U}\right)^{!}(M) \simeq \pi_{2 *} j_{U *} j_{U}^{!} \pi_{1}^{!}(M) .
\end{aligned}
$$

The adjunction triangle corresponding to the open embedding $j_{U}$ and the closed embedding $i_{Z}$ gives rise to the triangles of Radon transformations

$$
\begin{gathered}
{ }^{!} \mathcal{R}(M) \longrightarrow{ }^{!} \mathcal{R}_{\text {cst }}(M) \longrightarrow{ }^{!} \mathcal{R}^{\circ}(M) \stackrel{+1}{\longrightarrow}, \\
{ }^{*} \mathcal{R}_{c}^{\circ}(M) \longrightarrow{ }^{*} \mathcal{R}_{\text {cst }}(M) \longrightarrow{ }^{*} \mathcal{R}(M) \stackrel{+1}{\longrightarrow},
\end{gathered}
$$

where the second triangle is dual to the first.

We now introduce a family of Laurent polynomials defined on $T \times \Lambda:=\left(\mathbb{C}^{*}\right)^{d} \times \mathbb{C}^{n}$ using the columns of the matrix $A$; more precisely, we put

$$
\varphi_{A}: T \times \Lambda \longrightarrow V=\mathbb{C}_{\lambda_{0}} \times \Lambda, \quad\left(t_{1}, \ldots, t_{d}, \lambda_{1}, \ldots, \lambda_{n}\right) \longmapsto\left(-\sum_{i=1}^{n} \lambda_{i} \underline{t}^{\underline{a}}, \lambda_{1}, \ldots, \lambda_{n}\right) .
$$

The following theorem of [Rei14] constructs a morphism between the Gauß-Manin system $\mathcal{H}^{0}\left(\varphi_{A,+} \mathcal{O}_{T \times \Lambda}\right)$, or its proper version $\mathcal{H}^{0}\left(\varphi_{A, \uparrow} \mathcal{O}_{T \times \Lambda}\right)$, and certain GKZ hypergeometric systems and identifies both with a corresponding Radon transform.

For this, we apply the triangle (5.3) to $M=g_{!} \mathbb{D}^{p} \mathbb{Q}_{T}^{H}$ and the triangle (5.4) to $M=g_{*}^{p} \mathbb{Q}_{T}^{H}$, where the map $g$ was defined by

$$
g: T \longrightarrow \mathbb{P}(W), \quad\left(t_{1}, \ldots, t_{d}\right) \longmapsto\left(1: \underline{t}^{\underline{a}_{1}}: \cdots: \underline{t}^{\underline{a}}{ }\right) .
$$

TheOREM 5.1 ([Rei14, Lemma 1.11, Proposition 3.4]). Let $A=\left(\underline{a}_{1}, \ldots, \underline{a}_{n}\right) \in M(d \times n, \mathbb{Z})$ and $\widetilde{A}=\left(\underline{\widetilde{a}}_{0}, \underline{\widetilde{a}}_{1}, \ldots, \underline{\widetilde{a}}_{n}\right) \in M((d+1) \times(n+1), \mathbb{Z})$ be as above, and assume that $\widetilde{A}$ satisfies

(i) $\mathbb{Z} \widetilde{A}=\mathbb{Z}^{d+1}$,

(ii) $\mathbb{N} \widetilde{A}=\mathbb{R}_{\geqslant 0} \widetilde{A} \cap \mathbb{Z}^{d+1}$.

Then for every $\widetilde{\beta} \in \mathbb{N} \widetilde{A}$ and every $\widetilde{\beta}^{\prime} \in \operatorname{int}(\mathbb{N} \widetilde{A})$, we have

$$
\mathcal{M}_{\widetilde{A}}^{\widetilde{\beta}} \simeq \operatorname{DMod}\left(\mathcal{H}^{n+1}\left({ }^{*} \mathcal{R}_{c}^{\circ}\left(g_{*}{ }^{p} \mathbb{Q}_{T}^{H}\right)\right)\right) \quad \text { and } \quad \mathcal{M}_{\widetilde{A}}^{-\widetilde{\beta}^{\prime}} \simeq \operatorname{DMod}\left(\mathcal{H}^{-n-1}\left(! \mathcal{R}^{\circ}\left(g ! \mathbb{D}^{p} \mathbb{Q}_{T}^{H}\right)\right)\right) \text {. }
$$




\section{T. Reichelt And C. Sevenheck}

If we define

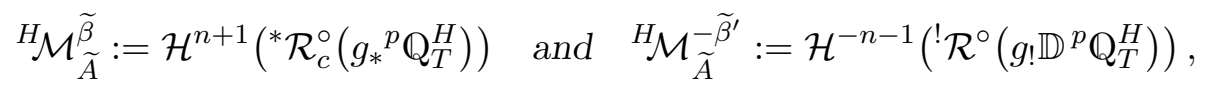

the following sequences of mixed Hodge modules are exact and dual to each other:

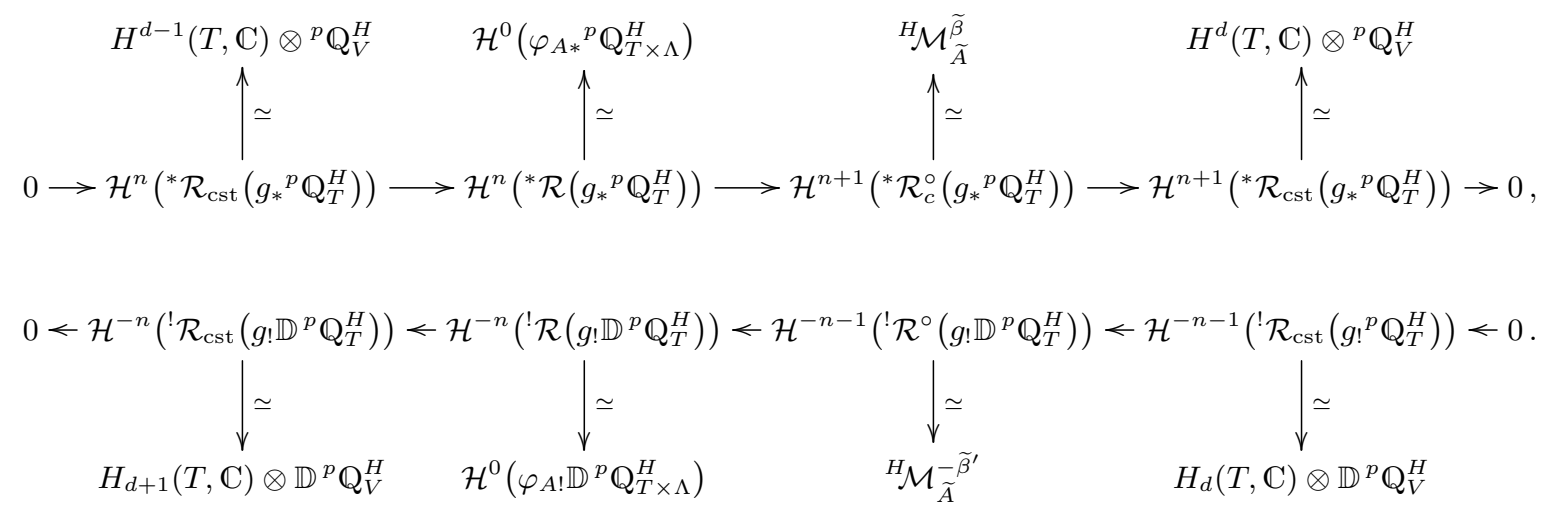

Proposition 5.2. Let $\widetilde{\beta} \in \mathbb{N} \widetilde{A}$ and $\widetilde{\beta}^{\prime} \in \operatorname{int}(\mathbb{N} \widetilde{A})$. There exists a natural morphism of mixed Hodge modules between $H_{\mathcal{M}}^{-\widetilde{\beta}^{\prime}}(-d-n)$ and $H_{\mathcal{M}}^{\widetilde{\beta}}$ which, on the underlying $\mathcal{D}_{V}$-modules, is given (up to multiplication with a non-zero constant) by

$$
\mathcal{M}_{\widetilde{A}}^{-\widetilde{\beta}^{\prime}} \longrightarrow \mathcal{M}_{\widetilde{A}}^{\widetilde{\beta}}, \quad P \longmapsto P \cdot \partial^{\widetilde{\beta}+\widetilde{\beta}^{\prime}}
$$

where $\partial^{\widetilde{\beta}+\widetilde{\beta}^{\prime}}:=\prod_{i=0}^{n} \partial_{\lambda_{i}}^{k_{i}}$ for any $\underline{k}=\left(k_{0}, \ldots, k_{n}\right)$ with $\widetilde{A} \cdot \underline{k}=\widetilde{\beta}+\widetilde{\beta}^{\prime}$.

Proof. First, notice that there is a natural morphism of mixed Hodge modules

$$
\mathcal{H}^{0}\left(\varphi_{A !} \mathbb{D}^{p} \mathbb{Q}_{T \times \Lambda}^{H}\right)(-d-n) \longrightarrow \mathcal{H}^{0}\left(\varphi_{A *}^{p} \mathbb{Q}_{T \times \Lambda}^{H}\right),
$$

which is induced by the morphism $\mathbb{D}^{p} \mathbb{Q}_{T \times \Lambda}^{H}(-d-n) \rightarrow{ }^{p} \mathbb{Q}_{T \times \Lambda}^{H}$. Using the isomorphisms in the second column, this gives a morphism

$$
\mathcal{H}^{-n}\left({ }^{!} \mathcal{R}\left(g_{!} \mathbb{D}^{p} \mathbb{Q}_{T}^{H}\right)\right)(-n-d) \longrightarrow \mathcal{H}^{n}\left({ }^{*} \mathcal{R}\left(g_{*}{ }^{p} \mathbb{Q}_{T}^{H}\right)\right) .
$$

Now, we can concatenate this with the morphisms

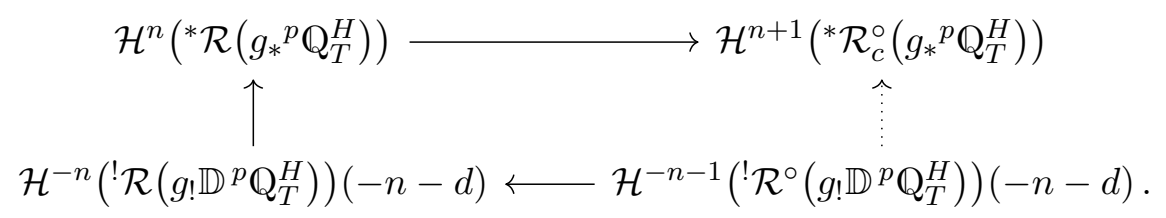

This gives the desired morphism of mixed Hodge modules between $H_{\mathcal{M}}^{-\widetilde{\beta}^{\prime}}(-d-n)$ and $H_{\mathcal{M}} \widetilde{\beta}_{\widetilde{A}}^{\widetilde{\beta}}$. Then, it follows from [RS17, Lemma 2.12] that the corresponding morphism of the underlying $\mathcal{D}_{V}$-modules is (up to multiplication by a non-zero constant) right multiplication with $\partial^{\widetilde{\beta}+\widetilde{\beta}^{\prime}}$.

We will now prove a partial generalization of Theorem 5.1 for non-integer $\beta$.

Proposition 5.3. With the notation as above, let $\widetilde{\beta}=\left(\beta_{0}, \beta\right) \in\left(\mathbb{Z} \times \mathbb{R}^{d}\right) \backslash \operatorname{sRes}(\widetilde{A})$; we have an isomorphism

$$
\operatorname{DMod}\left(\mathcal{H}^{n+1}\left({ }^{*} \mathcal{R}_{c}^{\circ}\left(g_{*}{ }^{p} \mathbb{C}_{T}^{\beta, H}\right)\right)\right) \simeq \mathcal{M}_{\widetilde{A}}^{\widetilde{\beta}} .
$$

This induces the structure of a complex mixed Hodge module on $\mathcal{M}_{\widetilde{A}}^{\widetilde{\beta}}$ which we call ${ }^{H_{\mathcal{M}}} \widetilde{\beta}_{\widetilde{A}}$. 


\section{Hypergeometric Hodge modules}

Proof. Consider the following commutative diagram with cartesian square:

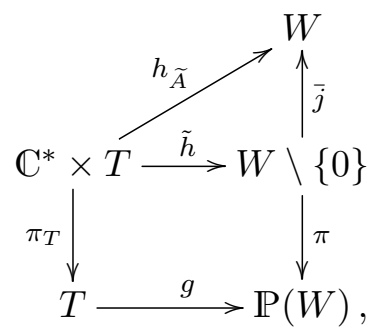

where $\pi_{T}$ is the projection to the first factor. We have

$$
\bar{j}_{+} \pi^{+} g_{+} \mathcal{O}_{T}^{\beta} \simeq \bar{j}_{+} \widetilde{h}_{+} \pi_{T}^{+} \mathcal{O}_{T}^{\beta} \simeq h_{\widetilde{A}+} \mathcal{O}_{\mathbb{C}^{*} \times T}^{(0, \beta)}[1] \simeq h_{+} \mathcal{O}_{\mathbb{C}^{*} \times T}^{\left(\beta_{0}, \beta\right)}[1]
$$

for every $\beta_{0} \in \mathbb{Z}$. Let ${ }^{*} \mathcal{R}_{c}^{\circ}: D_{r h}^{b}\left(\mathcal{D}_{X}\right) \rightarrow D_{r h}^{b}\left(\mathcal{D}_{X}\right)$ be the corresponding functor for $\mathcal{D}$-modules which is given by $M \mapsto \pi_{2+} j_{U} j_{U}^{\dagger} \pi_{1}^{\dagger} M$. We have an isomorphism

$$
{ }^{*} \mathcal{R}_{c}^{\circ}\left(g_{+} \mathcal{O}_{T}^{\beta}\right)[-n-1] \simeq \mathrm{FL}\left(\bar{j}_{+} \pi^{+} g_{+} \mathcal{O}_{T}^{\beta}[-1]\right) \simeq \mathrm{FL}\left(h_{\widetilde{A}+} \mathcal{O}_{\mathbb{C}^{*} \times T}^{\left(\beta_{0}, \beta\right)}\right) \simeq \mathcal{M}_{\widetilde{A}}^{\widetilde{\beta}},
$$

where the first isomorphism follows from [DE03, Proposition 1]. Notice that the various shifts occurring in the formulas above stem from a different (shifted) definition of the (exceptional) inverse image for $\mathcal{D}$-modules in [DE03].

\subsection{Integral transforms of twisted structure sheaves}

Unfortunately, the Radon transformation produces only GKZ systems with $\beta_{0} \in \mathbb{Z}$, as we can see in Proposition 5.3. To remedy this fact, we introduce an integral transformation which takes care of that by twisting with a kernel which depends on $\beta_{0}$.

Let $T=\left(\mathbb{C}^{*}\right)^{d}$ and $\widetilde{T}:=\left(\mathbb{C}^{*}\right)^{d+1}$ be tori with respective coordinates $t_{1}, \ldots, t_{d}$ and $t_{0}, \ldots, t_{d}$, and let $W=V=\mathbb{C}^{n+1}$ with respective coordinates $w_{0}, \ldots, w_{n}$ and $\lambda_{0}, \ldots, \lambda_{n}$. Consider the torus embedding with respect to the matrix $\widetilde{A}$

$$
h:=h_{\widetilde{A}}: \widetilde{T} \longrightarrow W, \quad\left(t_{0}, \ldots, t_{d}\right) \longmapsto\left(t_{0}, t_{0} \underline{t}^{\underline{a}}{ }^{1}, \ldots, t_{0} \underline{t}^{\underline{a}}{ }^{n}\right) .
$$

If $\widetilde{\beta} \notin \operatorname{sRes}(\widetilde{A})$, the GKZ system $\mathcal{M}_{\widetilde{A}}^{\widetilde{\beta}}$ is given by $\operatorname{FL}\left(h_{+} \mathcal{O}_{\widetilde{T}}^{\widetilde{\beta}}\right)$. Consider the maps

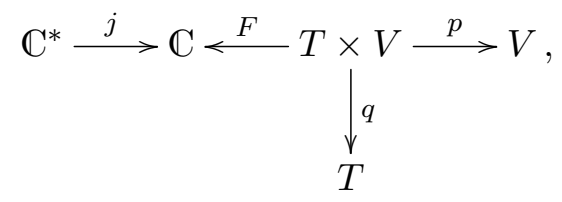

where $F$ is given by $\left(t_{1}, \ldots, t_{d}, \lambda_{0}, \ldots, \lambda_{n}\right) \mapsto \lambda_{0}+\sum_{i=1}^{n} \lambda_{i} \underline{\underline{a}}_{i}$, where $p_{1}$ and $p_{2}$ is the projection to the first and second factors, respectively, and where $j$ is the inclusion.

Proposition 5.3 showed that a GKZ system with integer $\beta_{0}$ can be expressed by a Radon transformation, generalizing a result in [Rei14]. The Radon transformation ${ }^{*} \mathcal{R}_{c}^{\circ}$ can be seen as an integral transform from $\mathbb{P}^{n}$ to $\mathbb{C}^{n+1}$ with kernel $j_{U !}{ }^{p} \mathbb{C}_{T}^{H}$. Now, in order to construct GKZ systems with general $\beta_{0}$, we could twist the kernel $j_{U !}{ }^{p} \mathbb{C}_{U}^{H}$, which means that instead of using the constant module ${ }^{p} \mathbb{C}_{U}^{H}$ on $U$, we could use a rank one local system on $U$ with monodromy $e^{2 \pi i \beta_{0}}$ (notice that $U$ has fundamental group isomorphic to $\mathbb{Z}$ ). However, for computational reasons, we use a slightly different approach. Instead of embedding the torus $T$ in $\mathbb{P}^{n}$ and considering an integral transform from $\mathbb{P}^{n}$ to $\mathbb{C}^{n+1}$, we directly define an integral transformation from $T$ to $V$ with a kernel depending on $\beta_{0}$ and $A$ (the matrix $A$ is encoded in the map $F$ ). 


\section{T. Reichelt And C. Sevenheck}

We prove in Proposition 5.4 that the outcome of this integral transformation applied to the $\mathcal{D}_{T}$-module $\mathcal{O}_{T}^{\beta}$ is indeed the GKZ system $\mathcal{M}_{\widetilde{A}}^{\widetilde{\beta}}$. Finally, we prove in Proposition 5.5 that this approach is compatible with the original approach using the Radon transform from Proposition 5.3.

The following proposition is a variant of a theorem of D'Agnolo and Eastwood [DE03]. It compares the Fourier-Laplace transform of the twisted structure sheaf under a torus embedding with an integral transform of the twisted structure on a smaller torus. The latter description is favorable since it naturally equips the GKZ system with the structure of a mixed Hodge module.

Proposition 5.4. Let $\widetilde{\beta}=\left(\beta_{0}, \beta\right) \notin \operatorname{sRes}(\widetilde{A}) ;$ then

$$
\mathcal{M}_{\widetilde{A}}^{\widetilde{\beta}} \simeq \mathrm{FL}\left(h_{+} \mathcal{O}_{\widetilde{T}}^{\widetilde{\beta}}\right) \simeq \mathcal{H}^{2 n+d+1}\left(p_{+}\left(q^{\dagger} \mathcal{O}_{T}^{\beta} \otimes_{\mathcal{O}} F^{\dagger}\left(j_{\dagger} \mathcal{O}_{\mathbb{C}^{*}}^{-\beta_{0}-1}\right)\right)\right) .
$$

Proof. Notice that the morphism $h: \widetilde{T} \longrightarrow \mathbb{C}^{n+1}$ factors as

$$
\widetilde{T} \stackrel{\widetilde{j}}{\longrightarrow} \mathbb{C} \times T \stackrel{k}{\longrightarrow} W,
$$

where $\widetilde{j}$ is the canonical embedding and $k$ is given by $\left(t_{0}, \ldots, t_{d}\right) \mapsto\left(t_{0}, t_{0} \underline{t}^{\underline{a_{1}}}, \ldots, t_{0} \underline{\underline{a}}_{n}\right)$. Consider the diagram
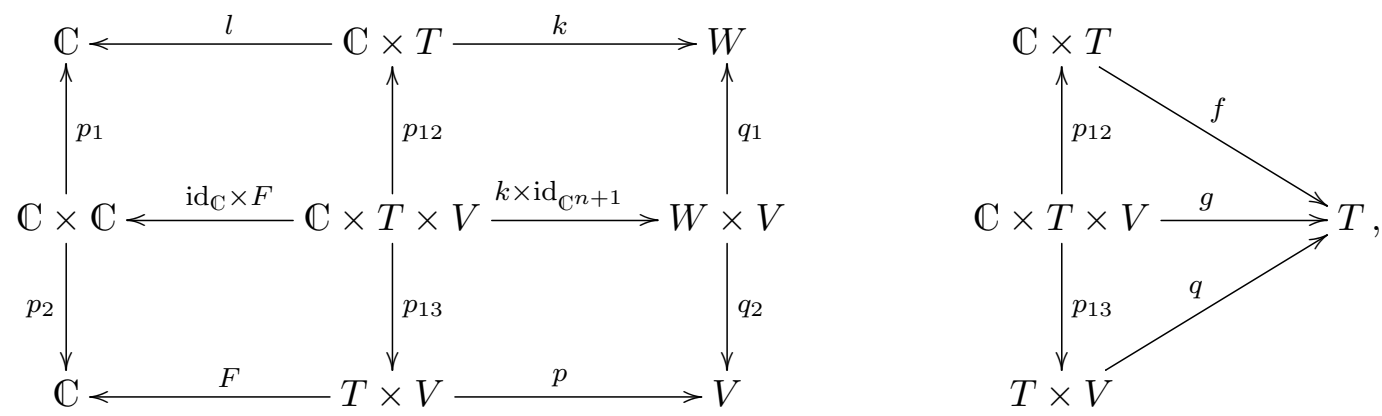

where the $p_{i j}$ are the projections to the factors $i$ and $j$, the maps $l, q, p_{1}, q_{1}$ are the projections to the first factor and the maps $f, g, p, p_{2}, q_{2}$ are the projection to the second factor.

We have that $\widetilde{j}_{+} \mathcal{O}_{\widetilde{T}}^{\widetilde{\beta}} \simeq j_{+} \mathcal{O}_{\mathbb{C}^{*}}^{\beta_{0}} \otimes \mathcal{O}_{T}^{\beta} \simeq l^{+} j_{+} \mathcal{O}_{\mathbb{C}^{*}}^{\beta_{0}}[-d] \otimes f^{+} \mathcal{O}_{T}^{\beta}[-1]$; hence, we get the following isomorphisms:

$$
\begin{aligned}
& \operatorname{FL}\left(h_{+} \mathcal{O}_{\widetilde{T}}^{\widetilde{\beta}}\right) \\
& \simeq \operatorname{FL}\left(k_{+} \widetilde{j}_{+} \mathcal{O}_{\widetilde{T}}^{\widetilde{\beta}}\right) \\
& \simeq q_{2,+}\left(q_{1}^{+} k_{+} \widetilde{j}_{+} \mathcal{O}_{\widetilde{T}}^{\widetilde{\beta}} \otimes \mathcal{L}\right)[-n-1] \\
& \simeq q_{2,+}\left(q_{1}^{+} k_{+}\left(l^{+} j_{+} \mathcal{O}_{\mathbb{C}^{*}}^{\beta_{0}} \otimes f^{+} \mathcal{O}_{T}^{\beta}\right) \otimes \mathcal{L}\right)[-n-d-2] \\
& \simeq q_{2,+}\left((k \times \mathrm{id})_{+} p_{12}^{+}\left(l^{+} j_{+} \mathcal{O}_{\mathrm{C}^{*}}^{\beta_{0}} \otimes f^{+} \mathcal{O}_{T}^{\beta}\right) \otimes \mathcal{L}\right)[-n-d-2] \\
& \simeq q_{2,+}(k \times \mathrm{id})_{+}\left(p_{12}^{+}\left(l^{+} j_{+} \mathcal{O}_{\mathbb{C}^{*}}^{\beta_{0}} \otimes f^{+} \mathcal{O}_{T}^{\beta}\right) \otimes(k \times \mathrm{id})^{+} \mathcal{L}\right)[-2 d-2] \\
& \simeq q_{2,+}(k \times \mathrm{id})_{+}\left((\mathrm{id} \times F)^{+} p_{1}^{+} j_{+} \mathcal{O}_{\mathbb{C}^{*}}^{\beta_{0}} \otimes g^{+} \mathcal{O}_{T}^{\beta} \otimes(k \times \mathrm{id})^{+} \mathcal{L}\right)[-n-2 d-3] \quad g=f \circ p_{12}, \\
& l \circ p_{12}=p_{1} \circ(\mathrm{id} \times F) \\
& \simeq p_{+} p_{13,+}\left((\mathrm{id} \times F)^{+} p_{1}^{+} j_{+} \mathcal{O}_{\mathbb{C}^{*}}^{\beta_{0}} \otimes g^{+} \mathcal{O}_{T}^{\beta} \otimes(k \times \mathrm{id})^{+} \mathcal{L}\right)[-n-2 d-3] \\
& p \circ p_{13}=q_{2} \circ(k \times \text { id }) \\
& \simeq p_{+} p_{13,+}\left((\mathrm{id} \times F)^{+} p_{1}^{+} j_{+} \mathcal{O}_{\mathbb{C}^{*}}^{\beta_{0}} \otimes g^{+} \mathcal{O}_{T}^{\beta} \otimes(\mathrm{id} \times F)^{+} \mathcal{L}_{1}\right)[-3 n-2 d-3] \text {, }
\end{aligned}
$$




\section{Hypergeometric Hodge modules}

$$
\begin{aligned}
& \simeq p_{+} p_{13,+}\left(g^{+} \mathcal{O}_{T}^{\beta} \otimes(\mathrm{id} \times F)^{+}\left(p_{1}^{+} j_{+} \mathcal{O}_{\mathbb{C}^{*}}^{\beta_{0}} \otimes \mathcal{L}_{1}\right)\right)[-2 n-d-3], \\
& \simeq p_{+} p_{13,+}\left(p_{13}^{+} q^{+} \mathcal{O}_{T}^{\beta} \otimes(\mathrm{id} \times F)^{+}\left(p_{1}^{+} j_{+} \mathcal{O}_{\mathbb{C}^{*}}^{\beta_{0}} \otimes \mathcal{L}_{1}\right)\right)[-2 n-d-3] \\
& \simeq p_{+}\left(q^{+} \mathcal{O}_{T}^{\beta} \otimes p_{13,+}(\mathrm{id} \times F)^{+}\left(p_{1}^{+} j_{+} \mathcal{O}_{\mathbb{C}^{*}}^{\beta_{0}} \otimes \mathcal{L}_{1}\right)\right)[-2 n-d-2] \\
& \simeq p_{+}\left(q^{+} \mathcal{O}_{T}^{\beta} \otimes F^{+} p_{2,+}\left(p_{1}^{+} j_{+} \mathcal{O}_{\mathbb{C}^{*}}^{\beta_{0}} \otimes \mathcal{L}_{1}\right)\right)[-2 n-d-2] \\
& \simeq p_{+}\left(q^{+} \mathcal{O}_{T}^{\beta} \otimes F^{+} j_{\dagger} \mathcal{O}_{\mathbb{C}^{*}}^{-\beta_{0}-1}\right)[-2 n-d-1] \\
& \simeq p_{+}\left(q^{\dagger} \mathcal{O}_{T}^{\beta} \otimes F^{\dagger} j_{\dagger} \mathcal{O}_{\mathbb{C}^{*}}^{-\beta_{0}-1}\right)[2 n+d+1] .
\end{aligned}
$$

$$
\begin{aligned}
& g=q \circ p_{13} \\
& \text { projection formula } \\
& \text { base change } \\
& \mathrm{FL}\left(j_{+} \mathcal{O}_{\mathbb{C}^{*}}^{\beta_{0}}\right) \simeq j_{\dagger} \mathcal{O}_{\mathbb{C}^{*}}^{-\beta_{0}-1} \\
& q^{+} \simeq q^{\dagger}[2 n+2] \\
& F^{+} \simeq F^{\dagger}[2 n+2 d] \quad \square
\end{aligned}
$$

The isomorphism $\mathcal{M}_{\widetilde{A}}^{\widetilde{\beta}} \simeq \mathcal{H}^{2 n+d+1}\left(p_{+}\left(q^{\dagger} \mathcal{O}_{T}^{\beta} \otimes F^{\dagger} j_{\dagger} \mathcal{O}_{\mathbb{C}^{*}}^{-\beta_{0}-1}\right)\right)$, which holds for $\widetilde{\beta} \in \mathbb{R}^{d+1} \backslash$ sRes $(\widetilde{A})$, endows the GKZ system with the structure of a complex mixed Hodge module. We define the mixed Hodge module structure by

$$
H_{\mathcal{M}}^{\widetilde{\beta}}:=\mathcal{H}^{2 n+d+1}\left(p_{*}\left(q^{* p} \mathbb{C}_{T}^{\beta} \otimes F^{*} j^{p} \mathbb{C}_{\mathbb{C}^{*}}^{-\beta_{0}-1}\right)\right) .
$$

We now check that the Hodge module structure on $\mathcal{M}_{\widetilde{A}}^{\widetilde{\widetilde{\beta}}}$ induced by the definition (5.6) coincides with the one of Proposition 5.3 in the case $\beta_{0} \in \mathbb{Z}$.

Proposition 5.5. If $\beta_{0} \in \mathbb{Z}$ and $\left(\beta_{0}, \beta\right) \notin \operatorname{sRes}(\widetilde{A})$, then there is an isomorphism

$$
{ }^{*} \mathcal{R}_{c}^{\circ}\left(g_{*}^{p} \mathbb{C}_{T}^{H, \beta}\right)[n+1] \simeq p_{*}\left(q^{* p} \mathbb{C}_{T}^{\beta} \otimes F^{*} j_{!}^{p} \mathbb{C}_{\mathbb{C}^{*}}^{H,-\beta_{0}-1}\right)[2 n+d+1] .
$$

Proof. Consider the following commutative diagram whose squares are cartesian:

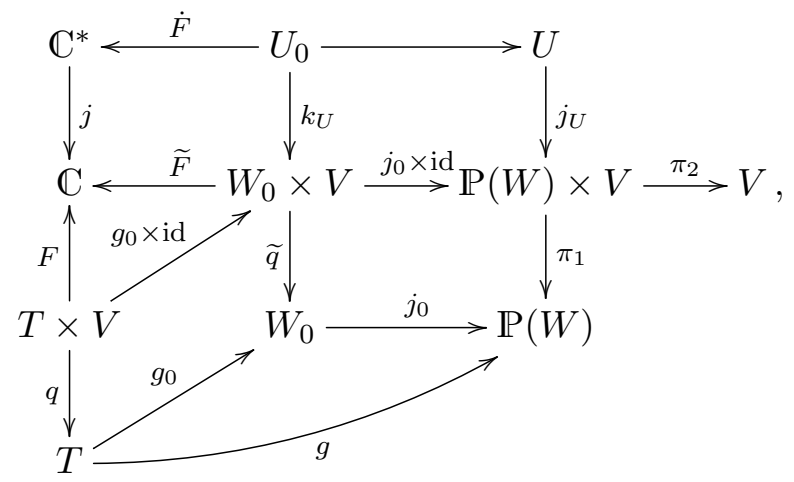

where $W_{0}:=\mathbb{P}(W) \backslash\left\{w_{0} \neq 0\right\}=\mathbb{C}^{n}$ with coordinates $w_{1}, \ldots, w_{n}$ and $U_{0}=U \cap W_{0}$. We denote by $p, p_{0}, \pi_{1}$ the projections to the first factor and by $\widetilde{F}$ the map $\left(w_{1}, \ldots, w_{n}, \lambda_{0}, \lambda_{n}\right) \mapsto$ $\lambda_{0}+\sum_{i=1}^{n} \lambda_{i} w_{i}$. We consider the coordinate change $\phi_{0}$ defined by

$$
\widetilde{t}_{k}=t_{k} \quad \text { for } \quad k=1, \ldots, d, \quad \widetilde{\lambda}_{0}=\lambda_{0}+\sum_{i=1}^{n} \lambda_{i} \underline{t}^{\underline{a}}=F \quad \text { and } \quad \widetilde{\lambda}_{i}=\lambda_{i} \quad \text { for } \quad i=1, \ldots, n
$$

on $T \times V$ and the coordinate change $\psi_{0}$ defined by

$$
\widetilde{w}_{j}=w_{j} \quad \text { for } \quad j=1, \ldots, n, \quad \widetilde{\lambda}_{0}=\lambda_{0}+\sum_{i=1}^{n} \lambda_{i} w_{j}=\widetilde{F} \quad \text { and } \quad \widetilde{\lambda}_{i}=\lambda_{i} \quad \text { for } \quad i=1, \ldots, n
$$

on $W_{0} \times V$. Notice that with respect to these coordinates, the maps $F$ and $\widetilde{F}$ are given by the coordinate function $\widetilde{\lambda}_{0}$. Let pr: $V \rightarrow \mathbb{C}$ be the projection $\left(\widetilde{\lambda}_{0}, \ldots, \widetilde{\lambda}_{n}\right) \mapsto \widetilde{\lambda}_{0}$. We also have 


\section{T. Reichelt And C. Sevenheck}

$\psi_{0} \circ\left(g_{0} \times \mathrm{id}\right) \circ \varphi_{0}^{-1}=g_{0} \times \mathrm{id}$, and the map $q$ factors as $\pi_{2} \circ j_{0} \circ(g \times \mathrm{id})$. Hence, we get

$$
\begin{aligned}
& p_{*}\left(q^{* p} \mathbb{C}_{T}^{H, \beta} \otimes F^{*} j_{!}^{p} \mathbb{C}_{\mathbb{C}^{*}}^{H,-\beta_{0}-1}\right)[2 n+d+1] \\
& \simeq p_{*}\left(q^{* p} \mathbb{C}_{T}^{H, \beta} \otimes F^{*} j_{!}^{p} \mathbb{C}_{\mathbb{C}^{*}}^{H}\right)[2 n+d+1] \quad \text { use } \beta_{0} \in \mathbb{Z} \\
& \simeq p_{*}\left({ }^{p} \mathbb{C}_{T}^{H, \beta} \otimes p r^{*} j_{!}^{p} \mathbb{C}_{\mathbb{C}^{*}}^{H}\right)[n+1] \quad F \text { is a projection after coordinate change } \\
& \simeq\left(\pi_{2} \circ\left(j_{0} \times \mathrm{id}\right) \circ\left(g_{0} \times \mathrm{id}\right)\right)_{*}\left({ }^{p} \mathbb{C}_{T}^{H, \beta} \otimes p r^{*} j_{!}^{p} \mathbb{C}_{\mathbb{C}^{*}}^{H}\right)[n+1] \quad p=\pi_{2} \circ\left(j_{0} \times \mathrm{id}\right) \circ\left(g_{0} \times \mathrm{id}\right) \\
& \simeq\left(\pi_{2} \circ\left(j_{0} \times \mathrm{id}\right)\right)_{*}\left(g_{0 *}{ }^{p} \mathbb{C}_{T}^{H, \beta} \otimes p r^{*} j_{!}^{p} \mathbb{C}_{\mathbb{C}^{*}}^{H}\right)[n+1] \\
& \simeq\left(\pi_{2} \circ\left(j_{0} \times \mathrm{id}\right)\right)_{*}\left(\widetilde{q}^{*} g_{0 *}{ }^{p} \mathbb{C}_{T}^{\beta} \otimes \widetilde{F}^{*} j_{!}^{p} \mathbb{C}_{\mathbb{C}^{*}}^{H}\right)[n+1] \\
& \simeq\left(\pi_{2} \circ\left(j_{0} \times \mathrm{id}\right)\right)_{*}\left(\widetilde{q}^{*} g_{0 *}{ }^{p} \mathbb{C}_{T}^{H, \beta} \otimes k_{U !} \dot{F}^{* p} \mathbb{C}_{\mathbb{C}^{*}}^{H}\right)[n+1] \quad \text { base change, } \dot{F} \text { and } \widetilde{F} \text { smooth } \\
& \simeq\left(\pi_{2} \circ\left(j_{0} \times \mathrm{id}\right)\right)_{*}\left(\widetilde{q}^{*} g_{0 *}{ }^{p} \mathbb{C}_{T}^{H, \beta} \otimes k_{U !}{ }^{p} \mathbb{C}_{U_{0}}^{H}\right)[n+1] \\
& \left.\simeq\left(\pi_{2} \circ\left(j_{0} \times \mathrm{id}\right)\right)_{*}\left(\left(j_{0} \times \mathrm{id}\right)\right)^{!} \pi_{1}^{*} g_{*}{ }^{p} \mathbb{C}_{T}^{H, \beta} \otimes k_{U !}^{p} \mathbb{C}_{U_{0}}^{H}\right)[n+1] \quad g=j_{0} \circ g_{0} \\
& \simeq \pi_{2 *}\left(\pi_{1}^{*} g_{*}^{p} \mathbb{C}_{T}^{H, \beta} \otimes\left(j_{0} \times \mathrm{id}\right)_{*} k_{U !}{ }^{p} \mathbb{C}_{U_{0}}^{H}\right)[n+1] \quad \text { projection formula } \\
& \stackrel{(*)}{\simeq} \pi_{2 *}\left(\pi_{1}^{*} g_{*}^{p} \mathbb{C}_{T}^{H, \beta} \otimes j_{U !}^{p} \mathbb{C}_{U}^{H}\right)[n+1] \\
& \simeq \pi_{2 *} j_{U !} j_{U}^{*} \pi_{1}^{*} g_{*}^{p} \mathbb{C}_{T}^{H, \beta}[n+1] \quad \text { projection formula } \\
& \simeq{ }^{*} \mathcal{R}_{c}^{\circ}\left(g_{*}^{p} \mathbb{C}_{T}^{H, \beta}\right)[n+1] \text {, }
\end{aligned}
$$

where the isomorphism $(*)$ follows from the fact that $\pi_{1}^{*} g_{*}{ }^{p} \mathbb{C}_{T}^{\beta}$ is localized along the divisor $\mathbb{P}(W) \backslash W_{0}$.

\subsection{Calculation in charts}

We saw in Subsection 5.2 that the GKZ system $\mathcal{M}_{\widetilde{A}}^{\widetilde{\beta}}$ can be expressed by an integral transformation from $T$ to $V$ with kernel $F^{*} j_{!}^{p} \mathbb{C}_{\mathbb{C}^{*}}^{-\beta_{0}-1}$. As a first step, we compute the Hodge filtration of an intermediate step in this integral transformation, namely $q^{* p} \mathbb{C}_{T}^{H, \beta^{u}} \otimes F_{u}^{*} j_{!}^{p} \mathbb{C}_{\mathbb{C}^{*}}^{H,-\beta_{0}-1}[2 n+d+1]$ (cf. Lemma 5.7). We do this by giving different presentations of the kernel (cf. Lemma 5.6) and by using several adapted coordinate systems on $T \times V$ indexed by a variable $u$ which goes from 0 to $n$. The reason for using these adapted coordinate systems (and not just one) is the fact that we can rewrite the intermediate step as a direct product whose factors are easy to compute. Since the projection $p: T \times V \rightarrow V$ is not proper, we have to extend the intermediate step to a partial compactification. Concretely, we are using the factorization

$$
T \times V \stackrel{g \times \text { id }}{\longrightarrow} \mathbb{P}(W) \times V \stackrel{\pi_{2}}{\longrightarrow} V
$$

of the projection $q$. Our goal in this section is to compute the underlying $\mathcal{D}$-module $\mathcal{N}$ of

$$
{ }^{H} \mathcal{N}:=\mathcal{H}^{2 n+d+1}(g \times \mathrm{id})_{*}\left(q^{* p} \mathbb{C}_{T}^{H, \beta} \otimes F^{*} j_{!}^{p} \mathbb{C}_{\mathbb{C}^{*}}^{H,-\beta_{0}-1}\right)
$$

together with its Hodge filtration on affine charts $W_{u}$ of $\mathbb{P}(W) \times V$. The different adapted coordinate systems are now used to compute the direct image of the intermediate step under the embedding $T \times V \rightarrow W_{u} \times V$, which is simply the restriction of ${ }^{H} \mathcal{N}$ to the affine chart $W_{u}$. It turns out that the underlying $\mathcal{D}$-module of this direct image is a direct product of a torus embedding with respect to a matrix $A_{u}$ and another rather simple module (cf. equation (5.8)). We use Theorem 4.17 to compute the Hodge filtration on the first factor in Proposition 5.9; the Hodge filtration on the second factor was computed in Remark 4.18. 


\section{Hypergeometric Hodge modules}

We define the map

$$
\begin{aligned}
& F_{u}: T \times V \longrightarrow \mathbb{C}, \\
&\left(t_{1}, \ldots, t_{d}, \lambda_{0}, \ldots, \lambda_{n}\right) \longmapsto \lambda_{u}+\sum_{\substack{i=0 \\
i \neq u}} \lambda_{i} \underline{t}^{\underline{a_{i}}-\underline{a}_{u}}=\left(\lambda_{0}+\sum_{i=1} \lambda_{i} \underline{\underline{a}}^{\underline{a}}\right) \cdot \underline{t}^{-\underline{a}_{u}}
\end{aligned}
$$

(notice that $F_{0}=F$ ). We need the following result.

LEMma 5.6. There is an isomorphism

$$
F^{*} j_{!}^{p} \mathbb{C}_{\mathbb{C}^{*}}^{H,-\beta_{0}-1} \simeq F_{u}^{*} j_{!}^{p} \mathbb{C}_{\mathbb{C}^{*}}^{H,-\beta_{0}-1}
$$

for $u=0, \ldots, n$.

Proof. For $u \in\{0, \ldots, n\}$ and $G:=\left(\mathbb{C}^{*}\right)^{d}$, consider the action

$$
\begin{aligned}
\mu_{u}: G \times T \times V & \longrightarrow T \times V, \\
\left(g_{1}, \ldots, g_{d}, t_{1}, \ldots, t_{d}, \lambda_{0}, \ldots, \lambda_{n}\right) & \longmapsto\left(t_{1}, \ldots, t_{d}, \lambda_{0} \underline{g}^{-\underline{a}_{u}}, \ldots, \lambda_{n} \underline{g}^{-\underline{a}_{u}}\right),
\end{aligned}
$$

and the action $G \times \mathbb{C} \rightarrow \mathbb{C}$ given by $\left(g_{1}, \ldots, g_{d}, t\right) \mapsto \underline{g}^{-a_{u}} \cdot t$. It is easy to see that the map $F: T \times V \rightarrow \mathbb{C}$ is equivariant with respect to this action. Let $i: T \rightarrow G \times T$ be the embedding $\left(t_{1}, \ldots, t_{d}\right) \mapsto\left(t_{1}, \ldots, t_{d}, t_{1}, \ldots, t_{d}\right)$. Since $j_{!}^{p} \mathbb{C}_{\mathbb{C}^{*}}^{H,-\beta_{0}-1}$ is a $G$-equivariant mixed Hodge module, the module $F^{!} j_{!}^{p} \mathbb{C}_{\mathbb{C}^{*}}^{H ;-\beta_{0}-1}$ is also $G$-equivariant. Let $p: G \times T \times V \rightarrow T \times V$ be the projection. We have isomorphisms

$$
F^{*} j_{!}^{p} \mathbb{C}_{\mathbb{C}^{*}}^{H,-\beta_{0}-1} \simeq i^{*} p_{2}^{*} F^{*} j_{!}^{p} \mathbb{C}_{\mathbb{C}^{*}}^{H,-\beta_{0}-1} \simeq i^{*} \mu_{u}^{*} F^{*} j_{!}^{p} \mathbb{C}_{\mathbb{C}^{*}}^{H,-\beta_{0}-1} \simeq F_{u}^{*} j_{!}^{p} \mathbb{C}_{\mathbb{C}^{*}}^{H,-\beta_{0}-1},
$$

where the second isomorphism follows from the $G$-equivariance of $F^{*} j_{!}^{p} \mathbb{C}_{T}^{H,-\beta_{0}-1}$.

We define a coordinate change $\phi_{u}$ by

$$
\widetilde{t}_{k}=t_{k}, \quad\left(\widetilde{\lambda}_{i}\right)_{i \neq u}=\left(\lambda_{i}\right)_{i \neq u} \quad \text { and } \quad \widetilde{\lambda}_{u}=\lambda_{u}+\sum_{i \neq u} \lambda_{i} \underline{t}^{\underline{a}} \underline{a}_{u}
$$

Denote by $C_{u} \in \mathrm{GL}(d+1, \mathbb{Z})$ the matrix

$$
C_{u}:=\left(\begin{array}{cccc}
1 & & & \\
-a_{1 u} & 1 & & \\
\vdots & & \ddots & \\
-a_{d u} & & & 1
\end{array}\right)
$$

and for $\widetilde{\beta}=\left(\beta_{0}, \beta\right) \in \mathbb{Z}^{d+1}$, define

$$
\widetilde{\beta}^{u}:=\left(\beta_{0}^{u}, \beta^{u}\right):=C_{u} \cdot \widetilde{\beta} .
$$

Notice that $\widetilde{\beta}^{0}=\widetilde{\beta}$ since $\underline{a}_{0}=0$.

Lemma 5.7. (i) With respect to the coordinates defined by $\phi_{u}$, the complex

$$
q^{* p} \mathbb{C}_{T}^{H, \beta^{u}} \otimes F_{u}^{*} j_{!}^{p} \mathbb{C}_{\mathbb{C}^{*}}^{H,-\beta_{0}-1}[2 n+d+1]
$$

is isomorphic to

$$
{ }^{p} \mathbb{C}_{T}^{H, \beta^{u}} \otimes \operatorname{pr}_{u}^{*} j_{!}^{p} \mathbb{C}_{\mathbb{C}^{*}}^{H,-\beta_{0}-1}[n],
$$

where $\operatorname{pr}_{u}: V \rightarrow \mathbb{C}$ is the projection $\left(\widetilde{\lambda}_{0}, \ldots, \widetilde{\lambda}_{n}\right) \mapsto \widetilde{\lambda}_{u}$. In particular, we have

$$
\mathcal{H}^{k}\left(q^{* p} \mathbb{C}_{T}^{H, \beta^{u}} \otimes F_{u}^{*} j_{!}^{p} \mathbb{C}_{\mathbb{C}^{*}}^{H,-\beta_{0}-1}\right)=0 \quad \text { for } \quad k \neq 2 n+d+1,
$$




\section{T. Reichelt And C. Sevenheck}

and the underlying $\mathcal{D}$-module of $\mathcal{H}^{2 n+d+1}\left(q^{*} p \mathbb{C}_{T}^{H, \beta^{u}} \otimes F_{u}^{*} j^{p} \mathbb{C}_{\mathbb{C}^{*}}^{H,-\beta_{0}-1}\right)$ is given by the exterior product

$$
\mathcal{D}_{T} /\left(\left(\partial_{\widetilde{t}_{k}} \widetilde{t}_{k}+\beta_{k}^{u}\right)_{k=1, \ldots, d}\right) \otimes \mathcal{D}_{V} /\left(\left(\partial_{\widetilde{\lambda}_{i}}\right)_{i \neq u}, \widetilde{\lambda}_{u} \partial_{\widetilde{\lambda}_{u}}-\beta_{0}\right)
$$

(ii) For $\alpha \in \mathbb{Z}^{d}$ and $u_{1}, u_{2} \in\{0, \ldots, n\}$, the map

$$
\mathcal{H}^{2 n+d+1}\left(q^{* p} \mathbb{C}_{T}^{H, \beta^{u_{1}}} \otimes F_{u_{1}}^{*} j_{!}^{p} \mathbb{C}_{\mathbb{C}^{*}}^{H,-\beta_{0}-1}\right) \longrightarrow \mathcal{H}^{2 n+d+1}\left(q^{* p} \mathbb{C}_{T}^{H, \beta^{u_{2}+\alpha}} \otimes F_{u_{2}}^{*} j_{!}^{p} \mathbb{C}_{\mathbb{C}^{*}}^{H,-\beta_{0}-1}\right)
$$

given by right multiplication with $\underline{t}^{\alpha}$ at the level of $\mathcal{D}_{V \times T}$-modules is an isomorphism.

Proof. Notice that the map $F_{u}$ is just the projection $\left(\left(\widetilde{t}_{k}\right)_{k=1, \ldots, s},\left(\widetilde{\lambda}_{i}\right)_{i \neq u}, \widetilde{\lambda}_{u}\right) \mapsto \tilde{\lambda}_{u}$ with respect to the new coordinates. This gives

$$
q^{* p} \mathbb{C}_{T}^{H, \beta^{u}} \otimes F_{u}^{*} j^{p} \mathbb{C}_{\mathbb{C}^{*}}^{H,-\beta_{0}-1}[2 n+d+1] \simeq{ }^{p} \mathbb{C}_{T}^{H, \beta^{u}} \otimes \operatorname{pr}_{u}^{*} j^{p} \mathbb{C}_{\mathbb{C}^{*}}^{H,-\beta_{0}-1}[n]
$$

(the shifts can be seen by noticing that $q^{*}[n+1], F^{*}[n+d]$ and $\operatorname{pr}_{u}^{*}[n]$ are exact). The rest is clear.

For the second point, we define coordinates. Let $\left(\left(\widetilde{t}_{k}\right)_{k=1, \ldots, d},\left(\widetilde{\lambda}_{i}\right)_{i=0, \ldots, n}\right)$ and $\left(\left(\bar{t}_{k}\right)_{k=1, \ldots, d}\right.$, $\left.\left(\bar{\lambda}_{i}\right)_{i=0, \ldots, n}\right)$ correspond to the maps $\phi_{u_{1}}$ and $\phi_{u_{2}}$, respectively. The coordinate change $\phi_{u_{2}} \circ \phi_{u_{1}}^{-1}$ is given by

$$
\bar{t}_{k}=\widetilde{t}_{k}, \quad \bar{\lambda}_{u_{1}}=\widetilde{\lambda}_{u_{1}}-\sum_{i \neq u_{1}} \widetilde{\lambda}_{i} \widetilde{t}^{\underline{a}_{i}-\underline{a}_{u_{1}}}, \quad \bar{\lambda}_{u_{2}}=\widetilde{\lambda}_{u_{1}} \widetilde{\widetilde{t}}^{\underline{a}_{u_{1}}-\underline{a}_{u_{2}}}, \quad \bar{\lambda}_{i}=\widetilde{\lambda}_{i}
$$

for $k=1, \ldots, d$ and $i \neq u_{1}, u_{2}$. We get the following transformations:

$$
\begin{aligned}
& \partial_{\tilde{\lambda}_{i}} \longmapsto \partial_{\bar{\lambda}_{i}}-\underline{\underline{t}}^{\underline{a}} \underline{-}^{-\underline{a}_{u_{1}}} \partial_{\bar{\lambda}_{u_{1}}} \equiv \partial_{\bar{\lambda}_{i}}, \\
& \widetilde{\lambda}_{u_{1}} \partial_{\bar{\lambda}_{u_{1}}}-\beta_{0} \longmapsto \bar{\lambda}_{u_{2}} \underline{\underline{t}} \underline{u}_{u_{2}}-\underline{a}_{u_{1}} \partial_{\bar{\lambda}_{u_{1}}}+\bar{\lambda}_{u_{2}} \partial_{\bar{\lambda}_{u_{2}}}-\beta_{0} \equiv \bar{\lambda}_{u_{2}} \partial_{\bar{\lambda}_{u_{2}}}-\beta_{0}, \\
& \partial_{\widetilde{\lambda}_{u_{2}}} \longmapsto-\underline{t}^{\underline{a}} u_{2}-\underline{a}_{u_{1}} \partial_{\bar{\lambda}_{u_{1}}} \\
& \partial_{\widetilde{t}_{k}} \widetilde{t}_{k}+\beta_{k}^{u_{1}} \longmapsto \partial_{\bar{t}_{k}} \bar{t}_{k}-\sum_{i \neq u_{1}}\left(a_{k i}-a_{k u_{1}}\right) \widetilde{\lambda}_{i} \underline{\underline{t}}^{\underline{a}_{i}-\underline{a}_{u_{1}}} \partial_{\bar{\lambda}_{u_{1}}}+\left(a_{k u_{1}}-a_{k u_{2}}\right) \bar{\lambda}_{u_{2}} \partial_{\bar{\lambda}_{u_{2}}}+\beta_{k}^{u_{1}} \\
& \equiv \partial_{\bar{t}_{k}} \bar{t}_{k}+\left(a_{k u_{1}}-a_{k u_{2}}\right) \bar{\lambda}_{u_{2}} \partial_{\bar{\lambda}_{u_{2}}}+\beta_{k}^{u_{1}} \\
& \equiv \partial_{\bar{t}_{k}} \bar{t}_{k}+\left(a_{k u_{1}}-a_{k u_{2}}\right) \beta_{0}+\beta_{k}^{u_{1}}=\partial_{\bar{t}_{k}} \bar{t}_{k}+\beta_{k}^{u_{2}},
\end{aligned}
$$

where $\equiv$ means equality modulo the ideal generated by the operators on the left-hand side. This shows that

$$
\mathcal{D}_{V} /\left(\left(\partial_{\widetilde{\lambda}_{i}}\right)_{i \neq u_{1}}, \widetilde{\lambda}_{u_{1}} \partial_{\widetilde{\lambda}_{u_{1}}}-\beta_{0}\right) \otimes \mathcal{D}_{T} /\left(\left(\partial_{\tilde{t}_{k}} \widetilde{t}_{k}+\beta_{k}^{u_{1}}\right)_{k=1, \ldots, d}\right)
$$

is actually equal to

$$
\mathcal{D}_{V} /\left(\left(\partial_{\bar{\lambda}_{i}}\right)_{i \neq u_{2}}, \bar{\lambda}_{u_{2}} \partial_{\bar{\lambda}_{u_{2}}}-\beta_{0}\right) \otimes \mathcal{D}_{T} /\left(\left(\partial_{\bar{t}_{k}} \bar{t}_{k}+\beta_{k}^{u_{2}}\right)_{k=1, \ldots, d}\right)
$$

after the change of coordinates $\phi_{u_{2}} \circ \phi_{u_{1}}^{-1}$. It is then easy to see that the map

$$
\begin{aligned}
& \mathcal{D}_{V \times T} /\left(\left(\partial_{\widetilde{\lambda}_{i}}\right)_{i \neq u_{2}}, \widetilde{\lambda}_{u_{2}} \partial_{\widetilde{\lambda}_{u_{2}}}-\beta_{0},\left(\partial_{\widetilde{t}_{k}} \widetilde{t}_{k}+\beta_{k}^{u_{2}}\right)_{k=1, \ldots, d}\right) \\
& \quad \rightarrow \mathcal{D}_{V} /\left(\left(\partial_{\bar{\lambda}_{i}}\right)_{i \neq u_{2}}, \bar{\lambda}_{u_{2}} \partial_{\bar{\lambda}_{u_{2}}}-\beta_{0},\left(\partial_{\bar{t}_{k}} \bar{t}_{k}+\beta_{k}^{u_{2}}+\alpha_{k}\right)_{k=1, \ldots, d}\right)
\end{aligned}
$$

is given by right multiplication with $\underline{\underline{t}}^{\alpha}$. This shows the second claim.

Let $\left(w_{0}: \cdots: w_{n}\right)$ be the homogeneous coordinates on $\mathbb{P}(W)$, and denote by $j_{u}: W_{u} \hookrightarrow \mathbb{P}(W)$ the chart $w_{u} \neq 0$ with coordinates $w_{i u}:=w_{i} / w_{u}$ for $i \neq u$. The map $g$ factors over the chart $W_{u}$ 


\section{Hypergeometric Hodge modules}

and gives rise to the map

$$
g_{u}: T \longrightarrow W_{u}, \quad\left(t_{1}, \ldots, t_{n}\right) \longmapsto\left(\underline{t}^{\underline{a}}-\underline{a}_{u}, \ldots, \underline{t}^{\underline{a}} \underline{-}^{-} \underline{a}_{u}\right) .
$$

We define the maps

$$
\widetilde{F}_{u}: W_{u} \times V \longrightarrow \mathbb{C}, \quad\left(w_{i u}\right)_{i \neq u} \longmapsto \lambda_{u}+\sum_{\substack{i=0 \\ i \neq u}}^{n} \lambda_{i} w_{i u} .
$$

As mentioned above, we would like to compute the restriction of $\mathcal{N}$ to the affine chart $W_{u} \times V$. For $u=0, \ldots, n$, we set

$$
\begin{aligned}
{ }^{H} \mathcal{N}_{u}:={ }^{H} \mathcal{N}_{\mid W_{u} \times V} & \simeq \mathcal{H}^{2 n+d+1}\left(g_{u} \times \mathrm{id}\right)_{*}\left(q^{* p} \mathbb{C}_{T}^{H, \beta^{u}} \otimes F_{u}^{*} j_{!}^{p} \mathbb{C}_{\mathbb{C}^{*}}^{H,-\beta_{0}-1}\right) \\
& \simeq \mathcal{H}^{n}\left(g_{u} \times \mathrm{id}\right)_{*}\left({ }^{p} \mathbb{C}_{T}^{H, \beta^{u}} \otimes \operatorname{pr}_{u}^{*} j_{!}^{p} \mathbb{C}_{\mathbb{C}^{*}}^{H,-\beta_{0}-1}\right) \\
& \simeq \mathcal{H}^{n}\left({ }^{p} g_{u *} \mathbb{C}_{T}^{H, \beta^{u}} \otimes \operatorname{pr}_{u}^{*} j^{p} \mathbb{C}_{\mathbb{C}^{*}}^{H,-\beta_{0}-1}\right) \\
& \simeq \mathcal{H}^{0}\left({ }^{p} g_{u *} \mathbb{C}_{T}^{H, \beta^{u}}\right) \otimes \mathcal{H}^{n}\left(\operatorname{pr}_{u}^{*} j^{p} \mathbb{C}_{\mathbb{C}^{*}}^{H,-\beta_{0}-1}\right) .
\end{aligned}
$$

We now apply the main result of Section 4 in order to compute the module $g_{u+} \mathcal{O}_{T}^{\beta^{u}}$ together with its corresponding Hodge filtration.

Notice that the embedding $g_{u}$ is given by the $d \times n$ matrix $A_{u}=\left(a_{k i}^{u}\right)$ with columns $\left(\underline{a}_{i}-\underline{a}_{u}\right)$ for $i \in\{0, \ldots, n\} \backslash\{u\}$. We need to check whether the matrices $A_{u}$ satisfy the conditions in Theorem 4.17. Recall that $\widetilde{\beta}^{u}:=\left(\beta_{0}^{u}, \beta^{u}\right):=C_{u} \cdot \widetilde{\beta}$.

Lemma 5.8. Assume $\mathbb{Z} \widetilde{A}=\mathbb{Z}^{d+1}$ and $\mathbb{N} \widetilde{A}=\mathbb{Z}^{d+1} \cap \mathbb{R}_{\geqslant 0} \widetilde{A}$. Then the matrices $A_{u}$ satisfy the conditions

(i) $\mathbb{Z} A_{u}=\mathbb{Z}^{d}$

(ii) $\mathbb{N} A_{u}=\mathbb{Z}^{d} \cap \mathbb{R}_{\geqslant 0} A_{u}$,

(iii) if $\widetilde{\beta} \in \mathfrak{A}_{\widetilde{A}}$, then $\beta^{u} \in \mathfrak{A}_{A_{u}}$.

Proof. Denote by $\widetilde{A}_{u}$ the $(d+1) \times(n+1)$ matrix with columns $\left(1, \underline{a}_{i}-\underline{a}_{u}\right)$ for $i \in\{0, \ldots, n\}$. We will first show the two properties for the matrix $\widetilde{A}_{u}$. Notice that we have $C_{u} \cdot \widetilde{A}=C_{u} \cdot \widetilde{A}_{0}=\widetilde{A}_{u}$. Since $C_{u}$ is a linear, invertible map, we get $C_{u}(\mathbb{Z} \widetilde{A})=\widetilde{A}_{u}, C_{u}(\mathbb{N} \widetilde{A})=\widetilde{A}_{u}$ and $C_{u}\left(\mathbb{R}_{\geqslant 0} \widetilde{A}\right)=\mathbb{R}_{\geqslant 0} \widetilde{A}_{u}$. Therefore, the two properties hold for $\widetilde{A}_{u}$ if and only if they hold for $\widetilde{A}$.

Denote by $p: \mathbb{Z}^{d+1} \rightarrow \mathbb{Z}^{d}$ the projection to the last $d$-coordinates. Since $p$ maps $\left(1, \underline{a}_{i}-\underline{a}_{u}\right)$ to $\underline{a}_{i}-\underline{a}_{u}$, it is easy to see that the first two properties also hold for $A_{u}$.

It follows easily from the definition that $\widetilde{\beta} \in \mathfrak{A}_{\widetilde{A}}$ if and only if $\widetilde{\beta}^{u} \in \mathfrak{A}_{\widetilde{A}_{u}}$. Hence, it is enough to show that $\widetilde{\beta}=\left(\beta_{0}, \beta\right) \in \mathfrak{A}_{\widetilde{A}}$ implies $\beta \in \mathfrak{A}_{A}$. We first notice that there is a 1-1 correspondence between facets of $\mathbb{R}_{\geqslant 0} A$ and facets of $\mathbb{R}_{\geqslant 0} \widetilde{A}$ containing $\underline{\widetilde{a}}_{0}=(1,0, \ldots, 0)$ given by

$$
F \leftrightarrow \widetilde{F}=F+\mathbb{R}_{\geqslant 0} \cdot(1,0, \ldots, 0) .
$$

If $n_{F}$ is a primitive, inward-pointing normal vector of a facet $F$ of $\mathbb{R}_{\geqslant 0} A$, then the vector $n_{\widetilde{F}}:=$ $\left(0, n_{F}\right)$ is a primitive, inward-pointing normal vector of the corresponding facet $\widetilde{F}$ of $\mathbb{R}_{\geqslant 0} \widetilde{A}$. Since $\underline{\widetilde{c}}:=\sum_{i=0}^{n} \underline{\widetilde{a}}_{i}=(n+1, \underline{c})$, we have $e_{\widetilde{F}}=\left\langle n_{\widetilde{F}}, \underline{\widetilde{c}}\right\rangle=\left\langle\left(0, n_{F}\right),(n+1, \underline{c}\rangle=\left\langle n_{F}, \underline{c}\right\rangle=e_{F}\right.$. By 


\section{T. Reichelt And C. Sevenheck}

Definition (4.3), we get

$$
\begin{aligned}
\widetilde{\beta} \in \mathfrak{A}_{\widetilde{A}} & =\bigcap_{\widetilde{F} \text { facet }}\left\{\mathbb{R} \cdot \widetilde{F}-\left[0, e_{\widetilde{F}}\right) \cdot \underline{\widetilde{c}}\right\} \subset \bigcap_{\substack{\widetilde{F} \text { facet } \\
\underline{\underline{a}_{0}} \in \widetilde{F}}}\left\{\mathbb{R} \cdot \widetilde{F}-\left[0, e_{\widetilde{F}}\right) \cdot \underline{\widetilde{c}}\right\} \\
& \Rightarrow \beta \in \bigcap_{F \text { facet }}\left\{\mathbb{R} \cdot F-\left[0, e_{F}\right) \cdot \underline{c}\right\}=\mathfrak{A}_{A} .
\end{aligned}
$$

Denote the $\mathbb{Z}$-module of relations among the columns of $A_{u}$ by $\mathbb{L}_{A_{u}}$. In order to calculate the direct image of $\mathcal{O}_{T}^{\beta}$ under the map $g_{u}$, we use Theorem 4.17, where $A_{u}$ takes the role of the matrix $B$ in that theorem.

Proposition 5.9. Consider the $\mathcal{D}_{W_{u}}$-module $\check{\mathcal{M}}_{A_{u}}^{\beta^{u}}$ as defined in Definition 3.5, that is, $\check{\mathcal{M}}_{A_{u}}^{\beta^{u}}=$ $\mathcal{D}_{W_{u}} / \check{\mathcal{I}}_{A_{u}}^{\beta^{u}}$, where the left ideal $\check{\mathcal{I}}_{A_{u}}^{\beta^{u}}$ is generated by

$$
\check{\square}_{\underline{m} \in \mathbb{L}_{A_{u}}}=\prod_{i \neq u: m_{i}>0} w_{i u}^{m_{i}}-\prod_{i \neq u: m_{i}<0} w_{i u}^{-m_{i}}
$$

and the Euler vector fields

$$
\check{E}_{k}^{u}+\beta_{k}^{u}=\sum_{i \neq u} a_{k i}^{u} \partial_{w_{i u}} w_{i u}+\beta_{k}^{u}=\sum_{i \neq u}\left(a_{k i}-a_{k u}\right) \partial_{w_{i u}} w_{i u}+\beta_{k}^{u} .
$$

Then the direct image $g_{u+} \mathcal{O}_{T}^{\beta_{u}}$ is isomorphic to $\check{\mathcal{M}}_{A_{u}}^{\beta^{u}}$. Moreover, the Hodge filtration on $\check{\mathcal{M}}_{A_{u}}^{\beta^{u}}$ is the order filtration shifted by $n-d$; that is,

$$
F_{p+(n-d)}^{H} \check{\mathcal{M}}_{A_{u}}^{\beta^{u}}=F_{p}^{\text {ord }} \check{\mathcal{M}}_{A_{u}}^{\beta^{u}} .
$$

Proof. The statement follows from Theorem 4.17 and Lemma 5.8.

We now want to compute how the $\mathcal{D}$-modules $g_{u+} \mathcal{O}_{T}^{\beta^{u}}$ glue on their common domain of definition. Let $u_{1}, u_{2} \in\{0, \ldots, n\}$, and denote the intersection $W_{u_{1}} \cap W_{u_{2}}$ by $W_{u_{1} u_{2}}$. We fix $u_{1}, u_{2} \in\{0, \ldots, n\}$ with $u_{1}<u_{2}$. We have the following change of coordinates between the charts $W_{u_{1}}$ and $W_{u_{2}}$ :

$$
w_{i u_{1}}=w_{i u_{2}} w_{u_{1} u_{2}}^{-1} \quad \text { for } \quad i \neq u_{2} \quad \text { and } \quad w_{u_{2} u_{1}}=w_{u_{1} u_{2}}^{-1},
$$

which gives the following transformation rules for vector fields:

$$
w_{i u_{1}} \partial_{w_{i u_{1}}}=w_{i u_{2}} \partial_{w_{i u_{2}}} \quad \text { for } \quad i \neq u_{2} \quad \text { and } \quad w_{u_{2} u_{1}} \partial_{w_{u_{2} u_{1}}}=-\sum_{i \neq u_{2}} w_{i u_{2}} \partial_{w_{i u_{2}}} .
$$

These transformation rules define an algebra isomorphism

$$
\iota_{u_{1} u_{2}}: D_{W_{u_{1}}}\left[w_{u_{2} u_{1}}^{-1}\right] \rightarrow D_{W_{u_{2}}}\left[w_{u_{1} u_{2}}^{-1}\right] .
$$

The module of global sections $\Gamma\left(W_{u_{1} u_{2}}, g_{u_{1}+} \mathcal{O}_{T}^{\beta^{u_{1}}}\right)$ can be expressed as the quotient $D_{W_{u_{1}}}\left[w_{u_{2} u_{1}}^{-1}\right] / \dot{I}_{A_{u_{1}}}^{\beta^{u_{1}}}$, where $\dot{I}_{A_{u_{1}}}^{\beta^{u_{1}}} \subset D_{W_{u_{1}}}\left[w_{u_{2} u_{1}}^{-1}\right]:=\mathbb{C}\left[\left(w_{i u_{1}}\right)_{i \neq u_{1}}\right]\left[w_{u_{2} u_{1}}^{-1}\right] \otimes_{\mathbb{C}\left[\left(w_{i u_{1}}\right)_{i \neq u_{1}}\right]} D_{W_{u_{1}}}$ is the left ideal generated by

$$
\begin{aligned}
& \check{E}_{k}^{u_{1}}+\beta_{k}^{u_{1}}=\sum_{\substack{i=0 \\
i \neq u_{1}}}^{n} a_{k i}^{u_{1}} \partial_{w_{i u_{1}}} w_{i u_{1}}+\beta_{k}^{u_{1}}=\sum_{\substack{i=0 \\
i \neq u_{1}}}^{n} a_{k i}^{u_{1}} w_{i u_{1}} \partial_{w_{i u_{1}}}+\sum_{\substack{i=0 \\
i \neq u_{1}}}^{n} a_{k i}^{u_{1}}+\beta_{k}^{u_{1}}, \quad k=1, \ldots, d, \\
& \check{\square}_{\underline{m}}=\prod_{\substack{m_{i}>0 \\
i \neq u_{1}}} w_{i u_{1}}^{m_{i}}-\prod_{\substack{m_{i}<0 \\
i \neq u_{1}}} w_{i u_{1}}^{-m_{i}}, \quad \underline{m} \in \mathbb{L}_{A_{u_{1}}} .
\end{aligned}
$$




\section{Hypergeometric Hodge modules}

Let $\gamma^{u_{1}}:=\sum_{i \neq u_{1}} a_{k i}^{u_{1}}$; then $\dot{I}_{A_{u_{1}}}^{\beta^{u_{1}}-\gamma^{u-1}} \subset D_{W_{u_{1}}}\left[w_{u_{2} u_{1}}^{-1}\right]$ is the left ideal generated by

$$
\begin{aligned}
& \text { (1) } \sum_{\substack{i=0 \\
i \neq u_{1}}}^{n} a_{k i}^{u_{1}} w_{i u_{1}} \partial_{w_{i u_{1}}}+\beta_{k}^{u_{1}}, \quad k=1, \ldots, d, \\
& \text { (2) } \square_{l}=\prod_{\substack{l_{i}>0 \\
i \neq u_{1}}} w_{i u_{1}}^{l_{i}}-\prod_{\substack{l_{i}<0 \\
i \neq u_{1}}} w_{i u_{1}}^{-l_{i}}, \quad l \in \mathbb{L}_{A_{u_{1}}} .
\end{aligned}
$$

We get the following isomorphism of $D_{W_{u_{1}}}\left[w_{u_{2} u_{1}}^{-1}\right]$-modules:

$$
D_{W_{u_{1}}}\left[w_{u_{2} u_{1}}^{-1}\right] / \dot{I}_{A_{u_{1}}}^{\beta^{u_{1}}} \longrightarrow D_{W_{u_{1}}}\left[w_{u_{2} u_{1}}^{-1}\right] / \dot{I}_{A_{u_{1}}}^{\beta^{u_{1}}-\gamma^{u_{1}}}, \quad 1 \longmapsto \prod_{i \neq u_{1}} w_{i u_{1}}^{-1},
$$

which is the image of the isomorphism

$$
\mathcal{O}_{T}^{\gamma^{u_{1}+\beta_{k}^{u_{1}}-1} \longrightarrow \mathcal{O}_{T}^{\beta_{k}^{u_{1}}-1}}, \quad 1 \mapsto \underline{t}^{-\gamma^{u_{1}}}=\underline{t}^{-\sum_{i \neq u_{1}}\left(\underline{a}_{i}-\underline{a}_{u_{1}}\right)}
$$

under the functor $g_{u_{1}+}$ (cf. equation (4.14)). One obtains the same results for the chart $W_{u_{2}}$ by interchanging $u_{1}$ and $u_{2}$ above. Using the transformation rules (5.9), we can identify $D_{W_{u_{1}}}\left[w_{u_{2} u_{1}}^{-1}\right]$ with $D_{W_{u_{2}}}\left[w_{u_{1} u_{2}}^{-1}\right]$, which gives a well-defined map

$$
\iota_{u_{1} u_{2}}: D_{W_{u_{1}}}\left[w_{u_{2} u_{1}}^{-1}\right] / \dot{I}_{A_{u_{1}}}^{\beta^{u_{1}}-\gamma^{u_{1}}} \longrightarrow D_{W_{u_{2}}}\left[w_{u_{1} u_{2}}^{-1}\right] / \dot{I}_{A_{u_{2}}}^{\beta^{u_{2}-\gamma^{u_{2}}}} .
$$

We can now give an explicit expression for the gluing map between the various charts of the module $g_{+} \mathcal{O}_{T}^{\beta}$.

LEMmA 5.10. The isomorphism between $g_{u_{1}+} \mathcal{O}_{T}^{\beta^{u_{1}}}$ and $g_{u_{2}+} \mathcal{O}_{T}^{\beta^{u_{2}}}$ on their common domain of definition $W_{u_{1} u_{2}}=W_{u_{1}} \cap W_{u_{2}}$ is given by

$$
\begin{aligned}
\Gamma\left(W_{u_{1} u_{2}}, g_{u_{1}+} \mathcal{O}_{T}\right) \simeq D_{W_{u_{1}}}\left[w_{u_{2} u_{1}}^{-1}\right] / \dot{I}_{A_{u_{1}}}^{\beta^{u_{1}}} & \longrightarrow D_{W_{u_{2}}}\left[w_{u_{1} u_{2}}^{-1}\right] / \dot{I}_{A_{u_{2}}}^{\beta^{u_{2}}} \simeq \Gamma\left(W_{u_{1} u_{2}}, g_{u_{2}+} \mathcal{O}_{T}\right), \\
P & \longmapsto \iota_{u_{1} u_{2}}(P) w_{u_{1} u_{2}}^{n+1} .
\end{aligned}
$$

Proof. This follows easily from the discussion above by composing the three maps

$$
\begin{aligned}
D_{W_{u_{1}}}\left[w_{u_{2} u_{1}}^{-1}\right] / \dot{I}_{A_{u_{1}}}^{\beta^{u_{1}}} & \longrightarrow D_{W_{u_{1}}}\left[w_{u_{2} u_{1}}^{-1}\right] / \dot{I}_{A_{u_{1}}}^{\beta^{u_{1}}-\gamma^{u_{1}}} \stackrel{\iota}{\longrightarrow} D_{W_{u_{2}}}\left[w_{u_{1} u_{2}}^{-1}\right] / \dot{I}_{A_{u_{2}}}^{\beta^{u_{2}}-\gamma^{u_{2}}} \\
& \longrightarrow D_{W_{u_{2}}}\left[w_{u_{1} u_{2}}^{-1}\right] / \dot{I}_{A_{u_{2}}}^{\beta^{u_{2}}}
\end{aligned}
$$

and by using the simple computation

$$
\left(\prod_{i \neq u_{2}} w_{i u_{2}}\right) \cdot \iota\left(\prod_{i \neq u_{1}} w_{i u_{1}}^{-1}\right)=w_{u_{1} u_{2}}^{n+1} .
$$

Consider the following change of coordinates $\theta_{u}$ on $W_{u} \times V$ :

$$
\widetilde{\lambda}_{u}=\lambda_{u}+\sum_{\substack{j=0 \\ j \neq u}}^{n} \lambda_{j} w_{j u}, \quad \widetilde{\lambda}_{i}=\lambda_{i} \quad \text { and } \quad \widetilde{w}_{i u}=w_{i u}
$$

for $i=0, \ldots \underset{\lambda^{\prime}}{ }, n$ and $i \neq \underset{\widetilde{\lambda}}{\sim}$. Notice that $\theta_{u}^{-1} \circ\left(g_{u} \times \mathrm{id}\right) \circ \phi_{u}^{=} g_{u} \times$ id and $\widetilde{F}_{u}$ is just the projection $\left(\left(\widetilde{w}_{i u}\right)_{i \neq u}, \tilde{\lambda}_{0}, \ldots, \widetilde{\lambda}_{n}\right) \mapsto \widetilde{\lambda}_{u}$

Proposition 5.11. Consider the original coordinates $\left(\left(w_{i u}\right)_{i \neq u},\left(\lambda_{0}, \ldots, \lambda_{n}\right)\right)$ of $W_{u} \times V$. Then there is an isomorphism of $\mathcal{D}_{W_{u} \times V^{-m o d u l e s}} \mathcal{N}_{u} \simeq \mathcal{D}_{W_{u} \times V} / \mathcal{K}_{A_{u}}$, where $\mathcal{K}_{A_{u}}^{\widetilde{\beta}^{u}}$ is the left $\mathcal{D}_{W_{u} \times V^{-}}$ 


\section{T. Reichelt and C. Sevenheck}

ideal generated by the following classes of operators:

$$
\begin{aligned}
& \text { (1) } \sum_{\substack{i=0 \\
i \neq u}}^{n} a_{k i}^{u} \partial_{w_{i u}} w_{i u}-\sum_{i=1}^{n} a_{k i} \lambda_{i} \partial_{\lambda_{i}}+\beta_{k}, \\
& \text { (2) } \check{\square}_{\underline{m}}=\prod_{\substack{m_{i}>0 \\
i \neq u}} w_{i u}^{m_{i}}-\prod_{\substack{m_{i}<0 \\
i \neq u}} w_{i u}^{-m_{i}}, \quad \underline{m} \in \mathbb{L}_{A_{u}}, \\
& \text { (3) } \partial_{\lambda_{i}}-w_{i u} \partial_{\lambda_{u}}, \quad i=0, \ldots, n \quad \text { and } i \neq u, \\
& \text { (4) } \sum_{j=0}^{n} \lambda_{j} \partial_{\lambda_{j}}-\beta_{0} .
\end{aligned}
$$

Moreover, for $\widetilde{\beta}=\left(\beta_{0}, \beta\right) \in \mathfrak{A}_{\widetilde{A}}$ and $\beta_{0} \in(-1,0]$, we have

$$
F_{p+(n-d)}^{H} \mathcal{N}_{u} \simeq F_{p}^{\text {ord }} \mathcal{D}_{W_{u} \times V} / \mathcal{K}_{A_{u}}^{\widetilde{\beta}_{u}}
$$

Proof. Recall that $\mathcal{N}_{u}=\check{\mathcal{M}}_{A_{u}}^{\beta^{u}} \otimes \mathcal{D}_{V} /\left(\left(\partial_{\widetilde{\lambda}_{i}}\right)_{i \neq u}, \widetilde{\lambda}_{u} \partial_{\widetilde{\lambda}_{u}}-\beta_{0}\right)=\mathcal{D}_{W_{u} \times V} / \widetilde{\mathcal{K}}_{A_{u}}^{\widetilde{\beta}^{u}}$, where

$$
\widetilde{\mathcal{K}}_{A_{u}}^{\widetilde{\beta}^{u}}=\left(\left(\check{E}_{k}^{u}+\beta_{k}^{u}\right)_{k=1, \ldots, d},\left(\check{\square}_{\underline{m}}\right)_{\underline{m} \in \mathbb{L}_{A_{u}}},\left(\partial_{\widetilde{\lambda}_{j}}\right)_{j \neq u},\left(\widetilde{\lambda}_{u} \partial_{\widetilde{\lambda}_{u}}-\beta_{0}\right)\right) \text {. }
$$

Using the coordinate transformation (5.10), we see that $\widetilde{\mathcal{K}}_{A_{u}}^{\widetilde{\beta}_{u}}$ is transformed into the ideal $\mathcal{K}_{A_{u}}^{\widetilde{\beta}}$ generated by the operators

$$
\begin{aligned}
& \sum_{\substack{i=0 \\
i \neq u}}^{n} a_{k i}^{u}\left(\partial_{w_{i u}}-\lambda_{i} \partial_{\lambda_{u}}\right) w_{i u}+\beta_{k}^{u}, \quad k=1, \ldots, d, \\
& \check{\square}_{\underline{m}}=\prod_{\substack{m_{i}>0 \\
i \neq u}} w_{i u}^{m_{i}}-\prod_{\substack{m_{i}<0 \\
i \neq u}} w_{i u}^{-m_{i}}, \quad \underline{m} \in \mathbb{L}_{A_{u}}, \\
& \partial_{\lambda_{i}}-w_{i u} \partial_{\lambda_{u}}, \quad i=0, \ldots, n \quad \text { and } \quad i \neq u, \\
& \left(\lambda_{u}+\sum_{\substack{j=0 \\
j \neq u}}^{n} \lambda_{j} w_{j u}\right) \partial_{\lambda_{u}}-\beta_{0} .
\end{aligned}
$$

The last operator can be rewritten (using the relations $\partial_{\lambda_{i}}-w_{i u} \partial_{\lambda_{u}}$, that is, the third class of operators) as

$$
\sum_{j=0}^{n} \lambda_{j} \partial_{\lambda_{j}}-\beta_{0} \equiv\left(\lambda_{u}+\sum_{\substack{j=0 \\ j \neq u}}^{n} \lambda_{j} w_{j u}\right) \partial_{\lambda_{u}}-\beta_{0}
$$

The Euler-type operators $\sum_{\substack{i=0 \\ i \neq u}}^{n} a_{k i}^{u}\left(\partial_{w_{i u}}-\lambda_{i} \partial_{\lambda_{u}}\right) w_{i u}$ can be further simplified by writing

$$
\begin{aligned}
\sum_{\substack{i=0 \\
i \neq u}}^{n} a_{k i}^{u}\left(\partial_{w_{i u}}-\lambda_{i} \partial_{\lambda_{u}}\right) w_{i u}+\beta_{k}^{u} & \equiv \sum_{\substack{i=0 \\
i \neq u}}^{n} a_{k i}^{u}\left(\partial_{w_{i u}} w_{i u}-\lambda_{i} \partial_{\lambda_{i}}\right)+\beta_{k}^{u} \\
& =\sum_{\substack{i=0 \\
i \neq u}}^{n} a_{k i}^{u} \partial_{w_{i u}} w_{i u}-\sum_{i=1}^{n} a_{k i} \lambda_{i} \partial_{\lambda_{i}}+a_{k u} \sum_{i=0}^{n} \lambda_{i} \partial_{\lambda_{i}}+\beta_{k}^{u}
\end{aligned}
$$




\section{Hypergeometric Hodge modules}

$$
\begin{aligned}
& \equiv \sum_{\substack{i=0 \\
i \neq u}}^{n} a_{k i}^{u} \partial_{w_{i u}} w_{i u}-\sum_{i=1}^{n} a_{k i} \lambda_{i} \partial_{\lambda_{i}}+a_{k u} \beta_{0}+\beta_{k}^{u} \\
& =\sum_{\substack{i=0 \\
i \neq u}}^{n} a_{k i}^{u} \partial_{w_{i u}} w_{i u}-\sum_{i=1}^{n} a_{k i} \lambda_{i} \partial_{\lambda_{i}}+\beta_{k},
\end{aligned}
$$

where the first equivalence follows by using the relation $\sum_{j=0}^{n} \lambda_{j} \partial_{\lambda_{j}} \equiv\left(\lambda_{u}+\sum_{\substack{j=0 \\ j \neq u}}^{n} \lambda_{j} w_{j u}\right) \partial_{\lambda_{u}}$ from above. Hence, we obtain the presentation $\mathcal{N}_{u} \simeq \mathcal{D}_{W_{u} \times V} / \mathcal{K}_{A_{u}}^{\widetilde{\beta}}$, and the statement on the Hodge filtration follows directly from Proposition 5.9.

\subsection{A Koszul complex}

In this subsection, we will construct a strict resolution of the filtered module $\left(\mathcal{N}_{u}, F^{H}\right)$. For this purpose, we first describe an alternative presentation of the ideal $\mathcal{K}_{A_{u}}^{\widetilde{\beta}} \subset \mathcal{D}_{W_{u} \times V}$. Let $A_{u}^{s}$ be the $(d+1) \times(2 n+1)$ matrix with columns $\left(0, \underline{a}_{0}-\underline{a}_{u}\right), \ldots,\left(0, \underline{a}_{u}-\underline{a}_{u}\right), \ldots,\left(0, \underline{a}_{n}-\right.$ $\left.\underline{a}_{u}\right),\left(1, \underline{a}_{0}\right), \ldots,\left(1, \underline{a}_{n}\right)$ (here, the symbol - means that the zero column $\left(0, \underline{a}_{u}-\underline{a}_{u}\right)$ is omitted). In other words, we have

$$
A_{u}^{s}=\left(\begin{array}{ccc|c|ccc}
0 & \ldots & 0 & 1 & 1 & \ldots & 1 \\
\hline & & & 0 & & \\
& A_{u} & & \vdots & & \\
& & & 0 & &
\end{array}\right) .
$$

In Lemma 5.13, we prove that the $\mathcal{D}$-module underlying $\mathcal{N}_{u}$ is isomorphic to a partial FourierLaplace-transformed GKZ system with respect to the matrix $A_{u}^{s}$ and parameter $\widetilde{\beta}_{u}$. With the help of the results in Subsection 3.1, we construct a $\mathcal{D}$-free strictly filtered resolution of the filtered module $\left(\mathcal{N}_{u}, F_{\bullet}^{H}\right)$ in Proposition 5.15.

As a first step, we prove some properties of the matrices $A_{u}^{s}$.

LEMmA 5.12. If, as before, $\mathbb{Z} \widetilde{A}=\mathbb{Z}^{d+1}$ and $\mathbb{N} \widetilde{A}=\mathbb{Z}^{d+1} \cap \mathbb{R}_{\geqslant 0} \widetilde{A}$ hold, then we have $\mathbb{Z} A_{u}^{s}=\mathbb{Z}^{d+1}$ and $\mathbb{N} A_{u}^{s}=\mathbb{Z}^{d+1} \cap \mathbb{R}_{\geqslant 0} A_{u}^{s}$.

Proof. From $\mathbb{Z} \widetilde{A}=\mathbb{Z}^{d+1}$, we conclude $\mathbb{Z} A_{u}^{s}=\mathbb{Z}^{d+1}$ since evidently $\mathbb{Z} \widetilde{A} \subset \mathbb{Z} A_{u}^{s}$. Hence, it remains to show that the semigroup $\mathbb{N} A_{u}^{s}$ is normal. We have

$C_{u} \cdot A_{u}^{s}=\left(\begin{array}{ccc|c|ccc}0 & \ldots & 0 & 1 & 1 & \ldots & 1 \\ \hline & & 0 & & \\ & A_{u} & \vdots & & A_{u}\end{array}\right)=:\left(\underline{\bar{a}}_{1}^{u}, \ldots, \underline{\bar{a}}_{n}^{u}, \underline{\widetilde{a}}_{0}^{u}, \underline{\widetilde{a}}_{1}^{u}, \ldots, \underline{\widetilde{a}}_{n}^{u}\right) \in M((d+1) \times(2 n+1), \mathbb{Z})$,

where $C_{u} \in \mathrm{GL}(d+1, \mathbb{Z})$ is the matrix already used in Lemma 5.8. It suffices to show the normality property for the semigroup $\mathbb{N}\left(C_{u} \cdot A_{u}^{s}\right)$ since $C_{u}$ is an invertible linear mapping, hence a homeomorphism. Suppose that we are given a linear combination

$$
\underline{v}=\sum_{i=1}^{n} \lambda_{i} \overline{\underline{a}}_{i}^{u}+\sum_{j=0}^{n} \mu_{j} \underline{\widetilde{a}}_{j}^{u} \in \mathbb{Z}^{d+1},
$$

where $\lambda_{i}, \mu_{j} \in \mathbb{R}_{\geqslant 0}$. Then $v=\sum_{i=1}^{n}\left(\lambda_{i}+\mu_{i}\right) \underline{a}_{i}^{u}+\underline{\widetilde{a}}_{0}^{u} \cdot\left(\sum_{j=0}^{n} \mu_{j}\right)$. Clearly, $\sum_{j=0}^{n} \mu_{j} \in \mathbb{N}$, and moreover, the vector $\sum_{i=1}^{n}\left(\lambda_{i}+\mu_{i}\right) \underline{\bar{a}}_{i}^{u}$ lies in $\mathbb{R}_{\geqslant 0} A_{u}$, but the latter semigroup is normal according 


\section{T. Reichelt And C. Sevenheck}

to Lemma 5.8. Hence, we have $\sum_{i=1}^{n}\left(\lambda_{i}+\mu_{i}\right) \bar{a}_{i}^{u} \in \mathbb{N} A_{u}$, and therefore $v \in \mathbb{N} \widetilde{A}_{u} \subset \mathbb{N}\left(C_{u} \cdot A_{u}^{s}\right)$, as required.

We now show that $\mathcal{N}_{u}$ can be interpreted as a partial Fourier-Laplace transformed GKZ system. For this, we consider the GKZ system $\mathcal{M}_{A_{u}^{s}}$ on $\hat{W}_{u} \times V$ with coordinates $\left(\hat{w}_{i u}\right)_{i \neq u}$, $\lambda_{0}, \ldots, \lambda_{n}$. Let $\mathrm{FL}_{\hat{W}_{u}}$ be the partial Fourier-Laplace transformation which interchanges $\partial_{\hat{w}_{i u}}$ with $\left(w_{i u}\right)_{i \neq u}$ and $\hat{w}_{i u}$ with $-\partial_{w_{i u}}$.

Lemma 5.13. Let $\stackrel{(\vee)}{\mathcal{I}} \widetilde{\beta}_{u}^{s}$ be the left $\mathcal{D}_{W_{u} \times V}$-ideal generated by the operators

$$
\stackrel{(\vee v)}{\square}(\underline{m}, \underline{l}):=\prod_{\substack{m_{i}>0 \\ i \neq u}} w_{i u}^{m_{i}} \prod_{\substack{l_{i}>0 \\ 0 \leqslant i \leqslant n}} \partial_{\lambda_{i}}^{l_{i}}-\prod_{\substack{m_{i}<0 \\ i \neq u}} w_{i u}^{-m_{i}} \prod_{\substack{l_{i}>0 \\ 0 \leqslant i \leqslant n}} \partial_{\lambda_{i}}^{-l_{i}},
$$

where $(\underline{m}, \underline{l})=\left(\left(m_{i}\right)_{i \neq u}, l_{0}, \ldots, l_{n}\right) \in \mathbb{L}_{A_{u}^{s}}$,

$$
\begin{gathered}
\stackrel{(\vee)}{E_{k}^{u}}-\beta_{k}:=-\sum_{\substack{i=0 \\
i \neq u}}^{n} a_{k i}^{u} \partial_{w_{i u}} w_{i u}+\sum_{i=1}^{n} a_{k i} \lambda_{i} \partial_{\lambda_{i}}-\beta_{k} \quad \text { for } k=1, \ldots, d \\
\text { and } \quad \stackrel{(\vee)}{E_{0}^{u}}-\beta_{0}:=\sum_{i=0}^{n} \lambda_{i} \partial_{\lambda_{i}}-\beta_{0} .
\end{gathered}
$$

Then we have $\stackrel{(\mathcal{V})}{\mathcal{I}_{A_{u}^{s}}^{s}}=\mathcal{K}_{A_{u}}^{\widetilde{\beta_{u}}}$, and hence the $\mathcal{D}_{W_{u} \times V}$-module $\mathcal{N}_{u}$ is isomorphic to $\mathcal{D}_{W_{u} \times V} / \stackrel{(V)}{I} \widetilde{\beta}_{A_{u}^{s}}^{s}$. In other words, we have an isomorphism $\mathcal{N}_{u} \simeq \mathrm{FL}_{\hat{W}_{u}} \mathcal{M}_{A_{u}^{s}}^{\widetilde{\beta}}$.

Proof. For the first statement, notice that $\stackrel{(\vee V)}{\square}_{(\underline{m}, 0)}$ equals the operator $\check{\square}_{\underline{m}}$ from the definition of the ideal $\mathcal{K}_{u}$. On the other hand, one can obtain all operators $\stackrel{(V)}{\square}_{(\underline{m}, \underline{l})}$ from the operators $\check{\square}_{\left(\underline{m}^{\prime}, 0\right)}$ using the relations $\partial_{\lambda_{i}}-w_{i u} \partial_{\lambda_{u}}$. The last statement follows by interchanging $\partial_{w_{i u}}$ with $-\hat{w}_{i u}$ and $w_{i u}$ with $\partial_{\hat{w}_{i u}}$ in the classes of operators of types (1), (2), (3) and (4) in the definition of the ideal $\mathcal{K}_{A_{u}}^{\widetilde{\beta_{u}}}$.

Notice that the operators $\stackrel{(\vee)}{E_{k}^{u}}$ are the same operators as in Proposition 5.11(1), but multiplied by -1 , which is useful for a Fourier-Laplace transformation that will be performed below.

In order to construct a strictly filtered resolution of $\mathcal{N}_{u}$, we use the theory of Euler-Koszul complexes, which we explained in Subsection 3.1. It will be applied to the $\mathcal{D}_{\hat{W}_{u} \times V}$-module $\mathcal{M}_{A_{u}^{s}}^{\widetilde{\beta_{u}}}$. As before, we work at the level of global sections.

Let $F_{\bullet}^{\hat{\omega}} D_{\hat{W}_{u} \times V}$ be the filtration on $D_{\hat{W}_{u} \times V}$ corresponding to the weight vector

$$
\begin{aligned}
& \hat{\omega}=\left(\left(\operatorname{weight}\left(\hat{w}_{i u}\right)\right)_{i \neq u},\left(\operatorname{weight}\left(\partial_{\hat{w}_{i u}}\right)\right)_{i \neq u}, \operatorname{weight}\left(\lambda_{0}\right), \ldots, \text { weight }\left(\lambda_{n}\right), \operatorname{weight}\left(\partial_{\lambda_{0}}\right), \ldots, \operatorname{weight}\left(\partial_{\lambda_{n}}\right)\right) \\
& :=(\underbrace{1, \ldots, 1}_{n \text {-times }}, \quad \underbrace{0, \ldots, 0}_{n \text {-times }}, \quad \underbrace{0, \ldots, 0}_{(n+1) \text {-times }}, \quad \underbrace{1, \ldots, 1}_{(n+1) \text {-times }}) .
\end{aligned}
$$

Notice that this filtration corresponds to the order filtration $F_{\bullet}^{\text {ord }} D_{W_{u} \times V}$ under the FourierLaplace transformation functor $\mathrm{FL}_{\hat{W}_{u}}$. We obtain a filtered resolution $\left(\left(K_{u}^{\bullet}, d\right), F^{\hat{\omega}}\right)$ of $M_{A_{u}^{s}}^{\widetilde{\beta}_{u}}$. Using Remark 3.3, we show that this resolution is strict. 


\section{Hypergeometric Hodge modules}

Lemma 5.14. The Euler-Koszul complex $\left(K_{u}^{\bullet}, F_{\bullet}^{\hat{\omega}}\right)$ is a resolution of $\left(M_{A_{u}^{s}}^{\widetilde{\beta}}, F_{\bullet}^{\hat{\omega}}\right)$ in the category of filtered $D_{\hat{W}_{u} \times V^{-m o d u l e s ~}}$ (with respect to the filtration $F_{\bullet}^{\hat{\omega}} D_{\hat{W}_{u} \times V}$ ); that is, we have a quasiisomorphism $K_{u}^{\bullet} \rightarrow M_{A_{u}^{s}}$, and the complex $K_{u}^{\bullet}$ is strictly filtered.

Proof. By Remark 3.3 above, it is enough to show that $H^{-i}\left(\operatorname{Gr}_{\bullet}^{F^{\hat{\omega}}} K_{u}^{\bullet}\right)=0$ for $i \geqslant 1$ and $H^{0}\left(\operatorname{Gr}_{\bullet}^{F^{\hat{\omega}}} K_{u}^{\bullet}\right) \simeq \mathrm{Gr}_{\bullet}^{F^{\hat{\omega}}} M_{A_{u}^{s}}$. Denote by $G D_{\hat{W}_{u} \times V}=\operatorname{Gr}_{\bullet}^{\hat{\omega}} D_{\hat{W}_{u} \times V}$ the associated graded object of $D_{\hat{W}_{u} \times V}$, by $\left(\hat{v}_{i u}\right)_{i \neq u}$ the symbol of $\left(\partial_{\hat{w}_{i u}}\right)_{i \neq u}$ and by $\mu_{j}$ the symbol of $\partial_{\lambda_{j}}$ in $G D_{\hat{W}_{u} \times V}$. Since $\square_{(\underline{m}, \underline{l})}$ is homogeneous in $\left(\partial_{\lambda_{j}}\right)$ and $\operatorname{ord}_{\hat{\omega}}\left(\partial_{\hat{w}_{i u}}\right)=0$ for all $i \neq u$, we have

$$
\operatorname{Gr}^{\hat{\omega}}\left(D_{\hat{W}_{u} \times V} / D_{\hat{W}_{u} \times V} J_{A_{u}^{s}}\right)=G D_{\hat{W}_{u} \times V} / J_{A_{u}^{s}}^{g},
$$

where $J_{A_{u}^{s}}^{g}$ is generated by

$$
\square_{(\underline{m}, \underline{l})}:=\prod_{\substack{m_{i}>0 \\ i \neq u}} \hat{v}_{i u}^{m_{i}} \prod_{\substack{l_{i}>0 \\ 0 \leqslant i \leqslant n}} \mu_{i}^{l_{i}}-\prod_{\substack{m_{i}<0 \\ i \neq u}} \hat{v}_{i u}^{-k_{i}} \prod_{\substack{l_{i}>0 \\ 0 \leqslant i \leqslant n}} \mu_{i}^{-l_{i}} .
$$

Notice that

$$
G D_{\hat{W}_{u} \times V} / J_{A_{u}^{s}}^{g} \simeq \mathbb{C}\left[\left(\hat{w}_{i u}\right)_{i \neq u}, \lambda_{0}, \ldots, \lambda_{n}\right] \otimes_{\mathbb{C}} \mathbb{C}\left[\mathbb{N} A_{u}^{s}\right] .
$$

The associated graded complex $\mathrm{Gr}^{\hat{\omega}} K_{u}^{\bullet}$ is isomorphic to a Koszul complex

$$
\operatorname{Gr}^{\hat{\omega}} K_{u}^{\bullet} \simeq \operatorname{Kos}\left(G D_{\hat{W}_{u} \times V} / \hat{J}_{A_{u}^{s}}^{g},\left({ }^{g} E_{k}^{u}\right)_{k=0, \ldots, d}\right),
$$

where ${ }^{g} E_{k}^{u}$ is defined by

$$
{ }^{g} \hat{E}_{k}^{u}:=\sum_{i \neq u} a_{k i}^{u} \hat{w}_{i u} \hat{v}_{i u}+\sum_{i=1}^{n} a_{k i} \lambda_{i} \mu_{i} \quad \text { for } k=1, \ldots, d \text { and } \quad{ }^{g} E_{0}^{u}:=\sum_{i=0}^{n} \lambda_{i} \mu_{i} .
$$

It is shown in [BGM15, Theorem 1.2] that the ${ }^{g} E_{k}^{u}$ are part of a system of parameters. Since $\mathbb{N} A_{u}^{s}$ is a normal semigroup (see Lemma 5.12 above), the ring $G D_{\hat{W}_{u} \times V} / J_{A_{u}^{s}}^{g}$ is Cohen-Macaulay. Hence, $\left({ }^{g} E_{k}^{u}\right)_{k=0, \ldots, d}$ is a regular sequence in $G D_{\hat{W}_{u} \times V} / J_{A_{u}^{s}}^{g}$. This shows that $H^{-i}\left(\mathrm{Gr}_{\bullet}^{\hat{\omega}} K_{u}^{\bullet}\right)=0$ for $i \geqslant 1$. On the other hand, it follows from [SST00, Theorem 4.3.5] that $H^{0}\left(\mathrm{Gr}_{\bullet}^{\hat{\omega}} K_{u}^{\bullet}\right)=$ $G D_{\hat{W}_{u} \times V} /\left(J_{A_{u}^{s}}^{g}+\left({ }^{g} E_{k}^{u}\right)_{k=0, \ldots, d}\right) \simeq \mathrm{Gr}_{\bullet}^{\hat{\omega}} M_{A_{u}^{s}}^{\widetilde{\beta}}$, as required.

As a consequence, we obtain the filtered resolution of $\mathcal{N}_{u}$ we are looking for. Let $\stackrel{(\vee)}{J_{A_{u}}^{s}}$ be the ideal in $D_{W_{u} \times V}$ generated by the box operators $\stackrel{(\vee V}{\square}_{(\underline{m}, \underline{l})}$ for $(\underline{m}, \underline{l}) \in \mathbb{L}_{A_{u}^{s}}$. Put

$$
\stackrel{(\vee)}{K}=\bigoplus_{u}^{-l}=\bigoplus_{0 \leqslant i_{1}<\cdots<i_{l} \leqslant d} D_{W_{u} \times V} / \stackrel{(\vee \vee}{J^{s}}{ }_{A_{u}^{s}} e_{i_{1}, \ldots, i_{l}},
$$

and define

$$
\stackrel{(\vee v)}{K}_{u}^{\bullet}:=\operatorname{Kos}\left(D_{W_{u} \times V} / \stackrel{(\vee v)}{J}_{A_{u}^{s}},\left(\stackrel{(\vee)}{E}{ }_{k}^{u}-\beta_{k}\right)_{k=0, \ldots, d}\right)
$$

where the $\stackrel{(\vee)}{E}_{k}^{u}$ denote the (pairwise commuting) endomorphisms of $D_{W_{u} \times V} / \stackrel{(\vee)}{J_{A_{u}^{s}}^{s}}$ induced from

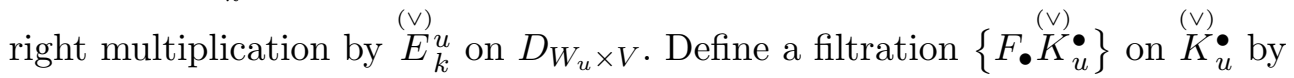

$$
F_{p} \stackrel{(\vee)}{K}_{u}^{l}:=\bigoplus_{0 \leqslant i_{1}<\cdots<i_{p} \leqslant d} F_{p-l+(n-d)}^{\text {ord }} D_{W_{u} \times V} / \stackrel{(\vee)}{J}_{A_{u}^{s}}
$$




\section{T. Reichelt And C. Sevenheck}

Proposition 5.15. We have a filtered quasi-isomorphism

$$
\left(K_{u}^{\bullet}, F_{\bullet}\right) \simeq\left(N_{u}, F_{\bullet}^{\text {ord }}\right) \simeq\left(N_{u}, F_{\bullet+(n-d)}^{H}\right) ;
$$

that is, the complex $\left(K_{u}^{\bullet}, F_{\bullet}\right)$ is a resolution of $\left(N_{u}, F_{\bullet+n-d}^{H}\right)$ in the category of filtered $D_{W_{u} \times V^{-}}$ modules.

Proof. The filtered quasi-isomorphism $\left(\stackrel{(\mathrm{V})}{K^{\bullet}}, F_{\bullet}\right) \simeq\left(N_{u}, F_{\bullet}^{\text {ord }}\right)$ is obtained by applying the Fourier-Laplace functor $\mathrm{FL}_{\hat{W}_{u}}$ to the (filtered) Euler-Koszul complex $\left(K_{u}^{\bullet}, F_{\bullet}^{\hat{\omega}}\right)$ from above (using Lemma 5.14). The second filtered (quasi-)isomorphism $\left(N_{u}, F_{\bullet}^{\text {ord }}\right) \simeq\left(N_{u}, F_{\bullet+(n-d)}^{H}\right)$ is just the content of Proposition 5.11.

\section{$5.5 \mathscr{R}$-modules}

In Subsection 5.4, we explicitly computed the filtered $\mathcal{D}$-module $\left(\mathcal{N}, F_{\bullet}^{H}\right)$ in the charts $W_{u} \times V$. Since the direct image of $\mathcal{N}$ under $\pi_{2}: \mathbb{P}(W) \times V \rightarrow V$ is the GKZ system we are looking for, we would just need to compute a filtered version of $R \pi_{2 *} D R_{\mathbb{P}(W) \times V / V}(\mathcal{N})$ using a Cech argument. It turns out that the theory of $\mathscr{R}$-modules is most suitable for this task. Hence, here we lift the results of Subsection 5.4 to the level of $\mathscr{R}$-modules. Following [Sab05], we first recall very briefly the basic notion of $\mathscr{R}$-modules and the Rees construction which provides a functor from the category of filtered $\mathcal{D}$-modules to that of $\mathscr{R}$-modules. In Proposition 5.17, we compute the corresponding Rees object of $\mathcal{N}_{u}$ and of its resolution (Lemma 5.18). We glue these resolutions in order to obtain a global resolution of the Rees object of $\mathcal{N}$ in Proposition 5.19.

Let $X$ be a smooth variety of dimension $n$. The order filtration of $\mathcal{D}_{X}$ gives rise to the Rees ring $R_{F} \mathcal{D}_{X}$. Given a filtered $\mathcal{D}_{X}$-module $\left(\mathcal{M}, F_{\bullet} \mathcal{M}\right)$, we construct the corresponding graded $R_{F} \mathcal{D}_{X}$-module $R_{F} \mathcal{M}:=\bigoplus_{k \in \mathbb{Z}} F_{k} \mathcal{M} z^{k}$. In local coordinates, the sheaf of rings $R_{F} \mathcal{D}_{X}$ is given by

$$
R_{F} \mathcal{D}_{X}=\mathcal{O}_{X}[z]\left\langle z \partial_{x_{1}}, \ldots, z \partial_{x_{n}}\right\rangle .
$$

Denote the product $X \times \mathbb{C}$ by $\mathscr{X}$. We will consider the sheaf

$$
\mathscr{R}_{\mathscr{X}}=\mathcal{O}_{\mathscr{X}} \otimes_{\mathcal{O}_{X}[z]} R_{F} \mathcal{D}_{X}
$$

and its ring of global sections

$$
\mathrm{R}_{\mathscr{X}}:=\Gamma\left(\mathscr{X}, \mathscr{R}_{\mathscr{X}}\right)=\mathcal{O}_{X}(X)[z]\left\langle z \partial_{x_{1}}, \ldots, z \partial_{x_{n}}\right\rangle .
$$

Given an $R_{F} \mathcal{D}_{X}$-module $R_{F} \mathcal{M}$, the corresponding $\mathscr{R}_{\mathscr{X}}$-module is

$$
\mathscr{M}:=\mathcal{O}_{\mathscr{X}} \otimes_{\mathcal{O}_{X}[z]} R_{F} \mathcal{M}
$$

This gives an exact functor $\mathscr{T}$ from the category of filtered $\mathcal{D}_{X}$-modules $M F\left(\mathcal{D}_{X}\right)$ to the category of $\mathscr{R}_{\mathscr{X}}$-modules $\operatorname{Mod}\left(\mathscr{R}_{\mathscr{X}}\right)$

$$
\mathscr{T}: M F\left(\mathcal{D}_{X}\right) \longrightarrow \operatorname{Mod}\left(\mathscr{R}_{\mathscr{X}}\right), \quad\left(\mathcal{M}, F_{\bullet} \mathcal{M}\right) \longmapsto \mathscr{M} .
$$

We denote the category of $\mathscr{R}_{\mathscr{X}}$-modules which are quasi-coherent $\mathcal{O}_{\mathscr{X}}$-modules by $\operatorname{Mod}_{q c}\left(\mathscr{R}_{\mathscr{X}}\right)$. We denote by $\Omega_{\mathscr{X}}^{1}=z^{-1} \Omega_{X \times \mathbb{C} / \mathbb{C}}^{1}$ the sheaf of algebraic 1 -forms on $\mathscr{X}$ relative to the projection $\mathscr{X} \rightarrow \mathbb{C}$ having at most a pole of order 1 along $z=0$. If we put $\Omega_{\mathscr{X}}^{k}=\wedge^{k} \Omega_{\mathscr{X}}^{1}$, we get a de Rham complex

$$
0 \longrightarrow \mathcal{O}_{\mathscr{X}} \stackrel{d}{\longrightarrow} \Omega_{\mathscr{X}}^{1} \stackrel{d}{\longrightarrow} \cdots \stackrel{d}{\longrightarrow} \Omega_{\mathscr{X}}^{n} \longrightarrow 0,
$$

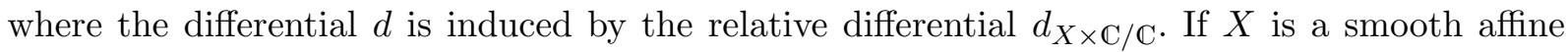
variety, we get the following equivalence of categories. 


\section{Hypergeometric Hodge modules}

Lemma 5.16. Let $X$ be a smooth affine variety. The functor

$$
\Gamma(\mathscr{X}, \bullet): \operatorname{Mod}_{q c}\left(\mathscr{R}_{\mathscr{X}}\right) \longrightarrow \operatorname{Mod}\left(R_{\mathscr{X}}\right)
$$

is exact and gives an equivalence of categories.

Proof. The proof is completely parallel to the $\mathcal{D}$-module case (see, for example, [HTT08, Proposition 1.4.4]).

One can also define a notion of direct image in the category of $\mathscr{R}$-modules. Since we only need the case of a projection, we will restrict ourselves to this special situation. Let $X$ and $Y$ be smooth algebraic varieties and $f: X \times Y \rightarrow Y$ be the projection to the second factor. As above, we have a relative de Rham complex $\Omega_{\mathscr{X} \times \mathscr{Y} / \mathscr{Y}}^{\bullet}=z^{-1} \Omega_{X \times Y \times \mathbb{C} / Y \times \mathbb{C}}^{\bullet}$ If $\mathscr{M}$ is an $\mathscr{R} \mathscr{X} \times \mathscr{Y}$-module, the relative de Rham complex $D R_{\mathscr{X} \times \mathscr{Y} / \mathscr{Y}}(\mathscr{M})$ is locally given by

$$
d(\omega \otimes m)=d \omega \otimes m+\sum_{i=1}^{n}\left(\frac{d x_{i}}{z} \wedge \omega\right) \otimes z \partial_{x_{i}} m,
$$

where $\left(x_{i}\right)_{1 \leqslant i \leqslant n}$ is a local coordinate of $X$. The direct image with respect to $f$ is then defined as

$$
f_{+} \mathscr{M}:=R f_{*} D R_{\mathscr{X} \times \mathscr{Y} / \mathscr{Y}}(\mathscr{M})[n] .
$$

Recall that for a filtered $\mathcal{D}$-module $\left(\mathcal{M}, F_{\bullet} \mathcal{M}\right)$, the direct image under $f$ is given by

$$
f_{+} \mathcal{M}=R f_{*}\left(0 \rightarrow \mathcal{M} \rightarrow \Omega_{X \times Y / Y}^{1} \otimes \mathcal{M} \rightarrow \cdots \rightarrow \Omega_{X \times Y / Y}^{n} \otimes \mathcal{M} \rightarrow 0\right)[n]
$$

together with its filtration

$$
F_{p} f_{+} \mathcal{M}=R f_{*}\left(0 \rightarrow F_{p} \mathcal{M} \rightarrow \Omega_{X \times Y / Y}^{1} \otimes F_{p+1} \mathcal{M} \rightarrow \cdots \rightarrow \Omega_{X \times Y / Y}^{n} \otimes F_{p+n} \mathcal{M} \rightarrow 0\right)[n] .
$$

Notice, however, that if $\left(\mathcal{M}, F_{\bullet} \mathcal{M}\right)$ underlies a mixed Hodge module on $X \times Y$, then the Hodge filtration on the cohomology modules of the direct image complex is not, in general, given by this definition unless $X$ is projective. It is a straightforward exercise to check that the functor $\mathscr{T}$ commutes with the direct image functor $f_{+}$.

We will apply this to the filtered $\mathcal{D}$-module $\left(\mathcal{N}, F_{\bullet}^{H}\right)$ as defined in equation (5.7) in order to compute $\mathcal{H}^{0} \pi_{2+} \mathcal{N} \simeq \mathcal{H}^{2 n+d+1}\left(p_{+}\left(q^{\dagger} \mathcal{O}_{T}^{\beta} \otimes F^{\dagger} j_{\dagger} \mathcal{O}_{\mathbb{C}^{*}}^{-\beta_{0}-1}\right)\right)$ together with its corresponding Hodge filtration. We denote the space $\mathbb{P}(W) \times V \times \mathbb{C}$ by $\mathscr{P} \times \mathscr{V}$. The corresponding $\mathscr{R}$-module is

$$
\mathscr{N}:=\mathscr{T}(\mathcal{N})=\mathcal{O}_{\mathscr{P} \times \mathscr{V}} \otimes_{\mathcal{O}_{\mathbb{P}(W) \times V}[z]} R_{F^{H}} \mathcal{N}
$$

The direct image with respect to $\pi_{2}$ is then given by

$$
\pi_{2+\mathscr{N}} \simeq R \pi_{2 *}\left(0 \rightarrow \mathscr{N} \rightarrow \Omega_{\mathscr{P} \times \mathscr{V} / \mathscr{V}}^{1} \otimes \mathscr{N} \rightarrow \cdots \rightarrow \Omega_{\mathscr{P} \times \mathscr{V} / \mathscr{V}}^{n} \otimes \mathscr{N} \rightarrow 0\right)[n]
$$

Since this is rather hard to compute, we will replace the complex

$$
0 \rightarrow \mathscr{N} \rightarrow \Omega_{\mathscr{P} \times \mathscr{V} / \mathscr{V}}^{1} \otimes \mathscr{N} \rightarrow \cdots \rightarrow \Omega_{\mathscr{P} \times \mathscr{V} / \mathscr{V}}^{n} \otimes \mathscr{N} \rightarrow 0
$$

with a quasi-isomorphic one. For this, we will construct a resolution of $\mathscr{N}$. Let $\mathscr{W}_{u} \times \mathscr{V}:=W_{u} \times$ $V \times \mathbb{C}$, and denote the restriction of $\mathscr{N}$ to $\mathscr{W}_{u} \times \mathscr{V}$ by $\mathscr{N}_{u}$. We write $\mathrm{R}_{\mathscr{W}_{u} \times \mathscr{V}}=\Gamma\left(\mathscr{W}_{u} \times \mathscr{V}, \mathscr{R}_{\left.\mathscr{W}_{u} \times \mathscr{V}\right)}\right.$; then the module of global sections of $\mathscr{N}_{u}$ is the $\mathrm{R}_{\mathscr{W}_{u} \times \mathscr{V} \text {-module }}$

$$
\mathrm{N}_{u}:=\Gamma\left(\mathscr{W}_{u} \times \mathscr{V}, \mathscr{N}_{u}\right)
$$

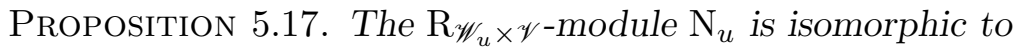

$$
z^{n-d} \cdot \mathrm{R}_{\mathscr{W}_{u} \times \mathscr{V}} / \mathrm{I}_{A_{u}^{s}}
$$




\section{T. Reichelt And C. Sevenheck}

where $\mathrm{I}_{A_{u}^{s}}$ is generated by

$$
\square_{(m, l)}:=\prod_{\substack{m_{i}>0 \\ i \neq u}} w_{i u}^{m_{i}} \prod_{\substack{l_{i}>0 \\ 0 \leqslant i \leqslant n}}\left(z \partial_{\lambda_{i}}\right)^{l_{i}}-\prod_{\substack{m_{i}<0 \\ i \neq u}} w_{i u}^{-m_{i}} \prod_{\substack{l_{i}<0 \\ 0 \leqslant i \leqslant n}}\left(z \partial_{\lambda_{i}}\right)^{-l_{i}},
$$

where $(m, l)=\left(\left(m_{i}\right)_{i \neq u}, l_{0}, \ldots, l_{n}\right) \in \mathbb{L}_{A_{u}^{s}}$,

$$
\begin{gathered}
\bar{E}_{k}^{u}-\beta_{k}:=-\sum_{\substack{i=0 \\
i \neq u}}^{n} a_{k i}^{u} z \partial_{w_{i u}} w_{i u}+\sum_{i=1}^{n} a_{k i} \lambda_{i} z \partial_{\lambda_{i}}-\beta_{k} \quad \text { for } \quad k=1, \ldots, d \\
\text { and } \bar{E}_{0}^{u}-\beta_{0}:=\sum_{i=0}^{n} \lambda_{i} z \partial_{\lambda_{i}}-\beta_{0} .
\end{gathered}
$$

Proof. This follows easily from Lemmas 5.13 and 5.16 .

We will now define a Koszul complex $\mathrm{K}_{u}^{\bullet}$ in the category of $\mathrm{R}_{u}$-modules which corresponds to the Koszul complex $\stackrel{(V)}{K}_{u}^{\bullet}$ alluded to above. Write $\mathrm{J}_{A_{u}^{s}}$ for the left ideal in $\mathrm{R}_{\mathscr{W}_{u} \times \mathscr{V}}$ generated by all operators $\bar{\square}_{(m, l)}$ for $(m, l) \in \mathbb{L}_{A_{u}^{s}}$. A computation similar to formula (3.1) shows that the maps

$$
\begin{aligned}
\mathrm{R}_{\mathscr{W}_{u} \times \mathscr{V}} / \mathrm{J}_{A_{u}^{s}} & \longrightarrow \mathrm{R}_{\mathscr{W}_{u} \times \mathscr{V}} / \mathrm{J}_{A_{u}^{s}}, \\
P & \longmapsto P \cdot\left(\bar{E}_{k}^{u}-\beta_{k}\right) \quad \text { for } \quad k=0, \ldots, d
\end{aligned}
$$

are well defined. Since $\left[\bar{E}_{k_{1}}^{u}-\beta_{k_{1}}, \bar{E}_{k_{2}}^{u}-\beta_{k_{2}}\right]=0$ for $k_{1}, k_{2} \in\{0, \ldots, d\}$, we can built a Koszul complex

$$
\mathrm{K}_{u}^{\bullet}:=\operatorname{Kos}\left(z^{n-d} \cdot \mathrm{R}_{\mathscr{W}_{u} \times \mathscr{V}} / \mathrm{J}_{A_{u}^{s}},\left(\cdot \bar{E}_{k}^{u}-\beta_{k}\right)_{k=0, \ldots, d}\right),
$$

whose terms are given by

$$
\begin{aligned}
& z^{n-2 d-1} \cdot \mathrm{R}_{\mathscr{W}_{u} \times \mathscr{V}} / \mathrm{J}_{A_{u}^{s}} \longrightarrow \cdots \longrightarrow \bigoplus_{i=1}^{n} z^{n-d-1} \cdot \mathrm{R}_{\mathscr{W}_{u} \times \mathscr{V}} / \mathrm{J}_{A_{u}^{s}} e_{1} \wedge \widehat{e}_{i} \wedge \cdots \wedge e_{d} \\
& \longrightarrow z^{n-d} \cdot \mathrm{R}_{\mathscr{W}_{u} \times \mathscr{V}} / \mathrm{J}_{A_{u}^{s}} e_{1} \wedge \cdots \wedge e_{d} .
\end{aligned}
$$

Lemma 5.18. The Koszul complex $\mathrm{K}_{u}^{\bullet}$ is a resolution of $\mathrm{N}_{u}$.

Proof. In order to prove the lemma, it is enough to apply the exact Rees functor $\mathscr{T}$ to the Koszul complex $\stackrel{(\sqrt{ })}{K}{ }_{u}^{\bullet}$ which is a strict resolution of $N_{u}$ in the category of filtered $D_{W_{u} \times V^{-m o d u l e s}}$ by Proposition 5.15.

We denote the corresponding resolution of $\mathscr{N}_{u}=\mathscr{N}_{\mid W_{u} \times V}$ by $\mathscr{K}_{u}^{\bullet}$. We are now able to construct a resolution of $\mathscr{N}$.

Proposition 5.19. There exists a resolution $\mathscr{K}^{\bullet}$ of $\mathscr{N}$ in the category of $\mathscr{R} \mathscr{P} \times \mathscr{V}$-modules which is locally given by $\Gamma\left(\mathscr{W}_{u} \times \mathscr{V}, \mathscr{K}^{\bullet}\right)=\mathrm{K}_{u}^{\bullet}$.

Proof. The resolution $\mathscr{K}^{\bullet}$ is constructed by providing glueing maps between the $\mathrm{R}_{\mathscr{W}_{u_{1} u_{2} \times \mathscr{V}^{-}}}$ modules

$$
\Gamma\left(\mathscr{W}_{u_{1} u_{2}} \times \mathscr{V}, \mathscr{K}_{u_{1}}^{\bullet}\right) \simeq \mathrm{K}_{u_{1}}^{\bullet}\left[w_{u 2 u 1}^{-1}\right] \longrightarrow \Gamma\left(\mathscr{W}_{u_{1} u_{2}} \times \mathscr{V}, \mathscr{K}_{u_{2}}^{\bullet}\right) \simeq \mathrm{K}_{u_{2}}^{\bullet}\left[w_{u 1 u 2}^{-1}\right]
$$

which are compatible with the glueing maps on

$$
\Gamma\left(\mathscr{W}_{u_{1} u_{2}} \times \mathscr{V}, \mathscr{N}_{u_{1}}\right) \simeq \mathrm{N}_{u_{1}}\left[w_{u 2 u 1}^{-1}\right] \longrightarrow \Gamma\left(\mathscr{W}_{u_{1} u_{2}} \times \mathscr{V}, \mathscr{N}_{u_{2}}\right) \simeq \mathrm{N}_{u_{2}}\left[w_{u 1 u 2}^{-1}\right]
$$




\section{Hypergeometric Hodge modules}

Notice that the latter maps are given by

$$
\mathrm{N}_{u_{1}}\left[w_{u 2 u 1}^{-1}\right] \longrightarrow \mathrm{N}_{u_{2}}\left[w_{u 1 u 2}^{-1}\right], \quad P \longmapsto \iota_{u_{1} u_{2}}(P) w_{u_{1} u_{2}}^{n+1},
$$

which follows from Lemma 5.10 and by tracing back the functors applied to $g_{u+} \mathcal{O}_{T}$. By using the same argument as in Lemma 5.10, we can show that the maps

$$
\mathrm{K}_{u_{1}}^{\bullet}\left[w_{u 2 u 1}^{-1}\right] \longrightarrow \mathrm{K}_{u_{2}}^{\bullet}\left[w_{u 1 u 2}^{-1}\right], \quad P \longmapsto \iota_{u_{1} u_{2}}(P) w_{u_{1} u_{2}}^{n+1}
$$

are well defined. We have to check that they give rise to a morphism of complexes. But this follows from the commutativity of the diagram

$$
\begin{gathered}
P \longmapsto \iota_{u_{1} u_{2}}(P) w_{u_{1} u_{2}}^{n+1} \\
\mathrm{R}_{\mathscr{W}_{u_{1}} \times \mathscr{V}} / \mathrm{J}_{u_{1}} \longrightarrow \mathrm{R}_{\mathscr{W}_{u_{2}} \times \mathscr{V}} / \mathrm{J}_{u_{2}} \\
\left.\mathrm{R}_{\mathscr{W}_{u_{1}} \times \mathscr{V}}^{u_{1}}\right|_{\mathrm{J}_{u_{1}} \longrightarrow} \\
P \mathrm{R}_{\mathscr{W}_{u_{2}} \times \mathscr{V}} / \mathrm{J}_{u_{2}}^{u_{2}-\beta_{k}} \\
P \longmapsto \\
\iota_{u_{1} u_{2}}(P) w_{u_{1} u_{2}}^{n+1}
\end{gathered}
$$

\subsection{A quasi-isomorphism}

In order to compute the direct image of the $\mathscr{R}$-module $\mathscr{N}$ under $\pi_{2}$, we have to deal with the relative de Rham complex $D R_{\mathscr{P} \times \mathscr{V} / \mathscr{V}}(\mathscr{N})$ (cf. formula (5.11)). In this subsection, we show in Proposition 5.20 that this complex is quasi-isomorphic to a complex $\mathscr{L}^{\bullet}$ which is the top cohomology (with respect to the de Rham differential) of the double complex $D R_{\mathscr{P} \times \mathscr{V} / \mathscr{V}}(\mathscr{K} \bullet)$. In Proposition 5.21, we give a local description of this complex $\mathcal{L}^{\bullet}$ on the charts $\mathscr{W}_{u} \times \mathscr{V}$.

As announced above, we now apply the relative de Rham functor $D R_{\mathscr{P} \times \mathscr{V} / \mathscr{V}}$ to the resolution $\mathscr{K}^{\bullet}$ and get a double complex $\Omega_{\mathscr{P} \times \mathscr{V} / \mathscr{V}}^{\bullet+n} \otimes \mathscr{K}^{\bullet}$ :

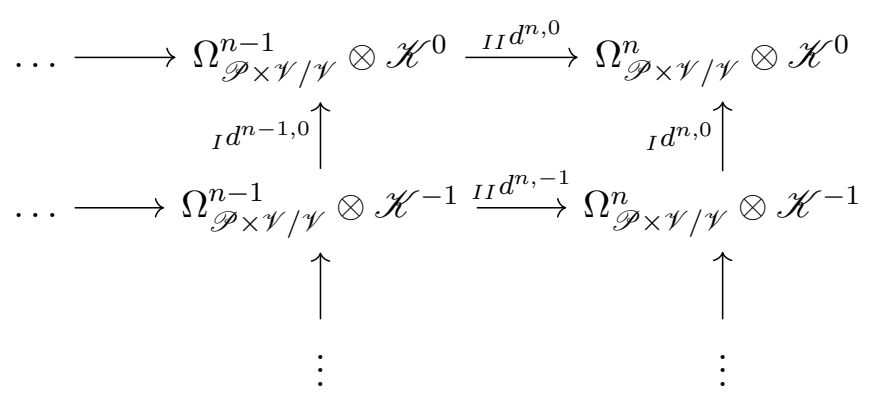

The corresponding total complex is denoted by $\operatorname{Tot}\left(\Omega_{\mathscr{P} \times \mathscr{V} / \mathscr{V}}^{\bullet+n} \otimes \mathscr{K}^{\bullet}\right)$.

Proposition 5.20. The natural morphisms of complexes

$$
\Omega_{\mathscr{P} \times \mathscr{V} / \mathscr{V}}^{\bullet+n} \otimes \mathscr{N} \longleftarrow \operatorname{Tot}\left(\Omega_{\mathscr{P} \times \mathscr{V} / \mathscr{V}}^{\bullet+n} \otimes \mathscr{K}^{\bullet}\right) \longrightarrow \Omega_{\mathscr{P} \times \mathscr{V} / \mathscr{V}}^{n} \otimes \mathscr{K}^{\bullet} /_{I I} d\left(\Omega_{\mathscr{P} \times \mathscr{V} / \mathscr{V}}^{n-1} \otimes \mathscr{K}^{\bullet}\right)=: \mathscr{L}^{\bullet}
$$

are quasi-isomorphisms. 


\section{T. Reichelt And C. Sevenheck}

Proof. Since the double complex $\Omega_{\mathscr{P} \times \mathscr{V} / \mathscr{V}}^{\bullet+n} \otimes \mathscr{K}^{\bullet}$ is bounded, we can associate with it two spectral sequences which both converge. The first one is given by taking cohomology in the vertical direction, which gives the ${ }_{I} E_{1}$-page of the spectral sequence. Since $\mathscr{K}^{\bullet}$ is a resolution of $\mathscr{N}$ and

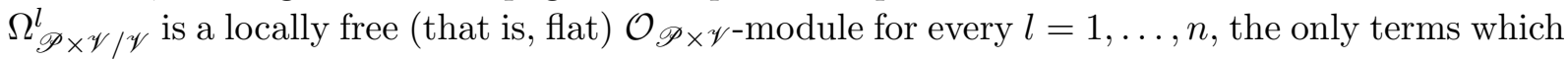
are non-zero are the ${ }_{I} E_{1}^{0, q}$-terms, which are isomorphic to $\Omega_{\mathscr{P} \times \mathscr{V} / \mathscr{V}}^{q+n} \otimes \mathscr{N}$. Hence, the first spectral sequence degenerates at the second page, which shows that $\Omega_{\mathscr{P} \times \mathscr{V} / \mathscr{V}}^{\bullet+n} \otimes \mathscr{N} \leftarrow \operatorname{Tot}\left(\Omega_{\mathscr{P} \times \mathscr{V} / \mathscr{V}}^{\bullet+n} \otimes \mathscr{K}^{\bullet}\right)$ is a quasi-isomorphism.

We now look at the second spectral sequence, which is given by taking cohomology in the horizontal direction. We claim that ${ }_{I I} E_{1}^{p, q}=0$ for $q \neq 0$. It is enough to check this locally on the charts $\mathscr{W}_{u} \times \mathscr{V}$ using, moreover, Lemma 5.16 at the level of global sections. Notice that the complex

$$
\Gamma\left(\mathscr{W}_{u} \times \mathscr{V}, \Omega_{\mathscr{P} \times \mathscr{V} / \mathscr{V}}^{\bullet+n} \otimes \mathscr{K}^{l}\right)
$$

is isomorphic to a direct sum of Koszul complexes $\operatorname{Kos}^{\bullet}\left(z^{-d-l} \mathrm{R}_{\mathscr{W}_{u} \times \mathscr{V}} / \mathrm{J}_{A_{u}^{s}}, \frac{1}{z}\left(z \partial_{w_{i u}} \cdot\right)_{i \neq u}\right)$, where each summand is given by

$$
z^{n-d-l} \mathrm{R}_{\mathscr{W}_{u} \times \mathscr{V}} / \mathrm{J}_{A_{u}^{s}} \longrightarrow \cdots \longrightarrow z^{-d-l} \mathrm{R}_{\mathscr{W}_{u} \times \mathscr{V}} / \mathrm{J}_{A_{u}^{s}} e_{1} \wedge \cdots \wedge e_{n}
$$

The quotient $\mathrm{R}_{\mathscr{W}_{u} \times \mathscr{V}} / \mathrm{J}_{A_{u}^{s}}$ can be written as

$$
\mathbb{C}\left[z,\left(z \partial_{w_{i u}}\right)_{i \neq u}\right] \otimes_{\mathbb{C}[z]}\left(\mathbb{C}\left[z, \lambda_{0}, \ldots, \lambda_{n},\left(w_{i u}\right)_{i \neq u}\right]\left\langle z \partial_{\lambda_{0}}, \ldots, z \partial_{\lambda_{n}}\right\rangle /\left(\left(\bar{\square}_{(m, l)}\right)_{(m, l) \in \mathbb{L}_{A_{u}^{s}}}\right)\right) .
$$

Since the operators $z \partial_{w_{i u}}$. act only on the first term in the tensor product, we immediately see that ${ }_{I I} E_{1}^{p, q}=0$ for $q \neq 0$.

The fact that $\operatorname{Tot}\left(\Omega_{\mathscr{P} \times \mathscr{V} / \mathscr{V}}^{\bullet+n} \otimes \mathscr{K}^{\bullet}\right) \rightarrow \mathscr{L}^{\bullet}$ is a quasi-isomorphism follows from the fact that ${ }_{I I} E^{p, q}=0$ for $q \neq 0$, that is, the second spectral sequence degenerates at the second page.

The next result is an explicit local description of the complex $\mathscr{L}^{\bullet}$.

Proposition 5.21. For any $u \in\{0, \ldots, n\}$, define the ring

$$
\mathrm{S}_{\mathscr{W}_{u} \times \mathscr{V}}:=\mathbb{C}\left[z, \lambda_{0}, \ldots, \lambda_{n},\left(w_{i u}\right)_{i \neq u}\right]\left\langle z \partial_{\lambda_{0}}, \ldots, z \partial_{\lambda_{n}}\right\rangle
$$

and denote by $\mathscr{S}$ the sheaf of rings on $\mathscr{P} \times \mathscr{V}$ which is locally given by

$$
\Gamma\left(\mathscr{W}_{u} \times \mathscr{V}, \mathscr{S}\right)=\mathrm{S}_{\mathscr{W}_{u} \times \mathscr{V}}
$$

with glueing maps

$$
\mathrm{S}_{\mathscr{W}_{u_{1}} \times \mathscr{V}}\left[w_{u_{2} u_{1}}^{-1}\right] \longrightarrow \mathrm{S}_{\mathscr{W}_{u_{2}} \times \mathscr{V}}\left[w_{u_{1} u_{2}}^{-1}\right], \quad P \longmapsto \iota_{u_{1} u_{2}}(P) .
$$

Denote by $\mathrm{J}_{A_{u}^{s}}$ the left $\mathrm{S}_{\mathscr{W}_{u} \times \mathscr{V}}$-ideal generated by the Box operators $\bar{\square}_{(m, l)}$ for $(m, l) \in \mathbb{L}_{A_{u}^{s}}$. Note that this is a slight abuse of notation, as the ideal generated by the same set of operators in the ring $\mathrm{R}_{\mathscr{W}_{u} \times \mathscr{V}}$ was also denoted by $\mathrm{J}_{A_{u}^{s}}$; it is justified by the fact that these generators do not contain the variables $z \partial_{w_{\text {iu }}}$. Then the complex $\mathscr{L}^{\bullet}$ is given locally by

$$
\Gamma\left(\mathscr{W}_{u} \times \mathscr{V}, \mathscr{L}^{\bullet}\right) \simeq \operatorname{Kos}^{\bullet}\left(z^{-d} \mathrm{~S}_{\mathscr{W}_{u} \times \mathscr{V}} / \mathrm{J}_{A_{u}^{s}},\left(\tilde{E}_{k}-\beta_{k}\right)_{k=0, \ldots, d}\right),
$$

whose terms are given by

$$
z^{-2 d-1} \mathrm{~S}_{\mathscr{W}_{u} \times \mathscr{V}} / \mathrm{J}_{A_{u}^{s}} \longrightarrow \cdots \longrightarrow z^{-d} \mathrm{~S}_{\mathscr{W}_{u} \times \mathscr{V}} / \mathrm{J}_{A_{u}^{s}} e_{1} \wedge \cdots \wedge e_{d}
$$

where

$$
\tilde{E}_{k}-\beta_{k}:=\sum_{i=1}^{n} a_{k i} \lambda_{i} z \partial_{\lambda_{i}}-\beta_{k} \quad \text { for } k=1, \ldots, d \quad \text { and } \quad \tilde{E}_{0}-\beta_{0}:=\sum_{i=0}^{n} \lambda_{i} z \partial_{\lambda_{i}}-\beta_{0} .
$$




\section{Hypergeometric Hodge modules}

Proof. It follows from Proposition 5.20 that the 0th cohomology of the complex $\left(\Omega_{\mathscr{P} \times \mathscr{V} / \mathscr{V}}^{\bullet+n} \otimes\right.$ $\left.\mathscr{K}^{p},{ }_{I I} d^{\bullet}, p\right)$ is a direct sum of terms of the form $H^{0}\left(z^{-d} \operatorname{Kos}^{\bullet}\left(\mathrm{R}_{\mathscr{W}_{u} \times \mathscr{V}} / \mathrm{J}_{A_{u}^{s}}, \frac{1}{z}\left(z \partial_{w_{i u}} \cdot\right)_{i \neq u}\right)\right)$. Taking the cokernel of left multiplication on $\mathrm{R}_{\mathscr{W}_{u} \times \mathscr{V}} / \mathrm{J}_{A_{u}^{s}}$ by $z \partial_{w_{i u}}$, we see that we have an isomorphism of $\mathrm{S}_{\mathscr{W}_{u} \times \mathscr{V} \text {-modules }}$

$$
H^{0}\left(z^{-d} \operatorname{Kos}^{\bullet}\left(\mathrm{R}_{\mathscr{W}_{u} \times \mathscr{V}} / \mathrm{J}_{A_{u}^{s}},\left(z \partial_{w_{i u}} \cdot\right)_{i \neq u}\right)\right) \simeq z^{-d} \mathrm{~S}_{\mathscr{W}_{u} \times \mathscr{V}} / \mathrm{J}_{A_{u}^{s}} .
$$

Hence, equation (5.12) follows.

The ideals $\mathrm{J}_{A_{u}^{s}}$ glue to an ideal $\mathscr{J} \subset \mathscr{S}$. Notice that the Euler vector fields $\left(\tilde{E}_{k}-\beta_{k}\right)_{k=0, \ldots, d}$ are global sections of $\mathscr{S}$. We recall from Proposition 5.19 that the glueing maps for $\Gamma\left(\mathscr{W}_{u} \times \mathscr{V}\right.$, $\left.\Omega_{\mathscr{P} \times \mathscr{V}}^{n} \otimes \mathscr{K}^{p}\right)$ are given by

$$
\bigwedge_{\substack{i=0 \\ i \neq u_{1}}}^{n} d w_{i u_{1}} \otimes P \longmapsto \bigwedge_{\substack{i=0 \\ i \neq u_{2}}}^{n} d w_{i u_{2}} \cdot\left(w_{u_{1} u_{2}}\right)^{-n-1} \otimes \iota_{u_{1} u_{2}}(P) w_{u_{1} u_{2}}^{n+1} .
$$

Since both powers of $w_{u_{1} u_{2}}$ on the right-hand side cancel when we consider the quotient $\mathscr{L}^{p}$, we see that

$$
\mathscr{L}^{\bullet} \simeq \operatorname{Kos}^{\bullet}\left(z^{-d} \mathscr{S} / \mathscr{J},\left(\tilde{E}_{k}-\beta_{k}\right)_{k=0, \ldots, d}\right) .
$$

Summarizing, Propositions 5.20 and 5.21 show that instead of computing the direct image (5.11), we can compute

$$
R \pi_{2 *}(\mathscr{L} \bullet) R \pi_{2 *}\left(z^{-d} \operatorname{Kos}^{\bullet}\left(\mathscr{S} / \mathscr{J},\left(\tilde{E}_{k}-\beta_{k}\right)_{k=0, \ldots, d}\right)\right) .
$$

\subsection{Computation of the direct image}

In this subsection, we continue our computation of $\pi_{2+} \mathscr{N} \simeq R \pi_{2 *}(\mathscr{L} \bullet)$. Because $\mathscr{V}$ is affine, it is enough to work at the level of global sections (cf. Lemma 5.16). We get

$$
\Gamma R \pi_{2 *}\left(\mathscr{L}^{\bullet}\right) \simeq R \Gamma R \pi_{2 *}\left(\mathscr{L}^{\bullet}\right) \simeq R \Gamma\left(\mathscr{L}^{\bullet}\right) \simeq R \Gamma\left(\operatorname{Kos}^{\bullet}\left(z^{-d} \mathscr{S} / \mathscr{J},\left(\tilde{E}-\beta_{k}\right)_{k=0, \ldots, d}\right)\right),
$$

where the first isomorphism follows from the exactness of $\Gamma(\mathscr{V}, \bullet)$. Hence, we have to consider the hypercohomology of a Koszul complex on $\mathscr{S} / \mathscr{J}$.

We will show that each term of this Koszul complex is $\Gamma$-acyclic. For this it is enough to show that $\mathscr{S} / \mathscr{J}$ is $\Gamma$-acyclic. In Proposition 5.22, we show that $\mathscr{S} / \mathscr{J}$ is acyclic and that its corresponding associated graded module has an easy description in terms of the "semi" R-module $S / J_{A^{s}}$ on $\mathbb{C}_{z} \times W \times V$ if all local cohomologies of $S / J_{A^{s}}$ with respect to the ideal $\left(w_{0}, \ldots, w_{n}\right)$ vanish. Finally, we show in Lemma 5.24 that the computation of these local cohomologies can be reduced to the computation of local cohomology groups of semigroup rings, which is a problem in commutative algebra which we tackle in Subsection 5.8.

Recall that $\mathscr{P} \times \mathscr{V}=\mathbb{C}_{z} \times \mathbb{P}(W) \times V$. We denote the space $\mathbb{C}_{z} \times W \times V$ by $\mathscr{W} \times \mathscr{V}$. Let

$$
\mathrm{S}:=\mathbb{C}\left[z, w_{0}, \ldots, w_{n}, \lambda_{0}, \ldots, \lambda_{n}\right]\left\langle z \partial_{\lambda_{0}}, \ldots, z \partial_{\lambda_{n}}\right\rangle,
$$

and consider the $\mathrm{S}$-module $\mathrm{S} / \mathrm{J}_{A^{s}}$, where the left ideal $\mathrm{J}_{A^{s}}$ is generated by

$$
\square_{(m, l)}=\prod_{m_{i}>0} w_{i}^{m_{i}} \prod_{l_{i}>0}\left(z \partial_{\lambda_{i}}\right)^{l_{i}}-\prod_{m_{i}<0} w_{i}^{-m_{i}} \prod_{l_{i}<0}\left(z \partial_{\lambda_{i}}\right)^{-l_{i}} \quad \text { for } \quad(m, l) \in \mathbb{L}_{A^{s}},
$$




\section{T. Reichelt And C. Sevenheck}

the matrix $A^{s}$ is given by

$$
A^{s}:=\left(\underline{a}_{0}^{s}, \ldots, \underline{a}_{n}^{s}, \underline{b}_{0}^{s}, \ldots, \underline{b}_{n}^{s}\right):=\left(\begin{array}{cccccccc}
1 & 1 & \ldots & 1 & 0 & 0 & \ldots & 0 \\
0 & 0 & \ldots & 0 & 1 & 1 & \ldots & 1 \\
0 & a_{11} & \ldots & a_{1 n} & 0 & a_{11} & \ldots & a_{1 n} \\
\vdots & \vdots & & \vdots & \vdots & \vdots & & \vdots \\
0 & a_{d 1} & \ldots & a_{d n} & 0 & a_{d 1} & \ldots & a_{d n}
\end{array}\right)
$$

and $\mathbb{L}_{A^{s}}$ is the $\mathbb{Z}$-module of relations among the columns of $A^{s}$. Notice that $\mathrm{S} / \mathrm{J}_{A^{s}}$ is $\mathbb{Z}$-graded by the degree of the $w_{i}$. Denote by $\mathrm{S}_{w_{u}}$ the localization of $\mathrm{S}$ with respect to $w_{u}$; then one easily sees that the degree zero part $\left[\mathrm{S}_{w_{u}} / \mathrm{J}_{A^{s}}\right]_{0}$ of $\mathrm{S}_{w_{u}} / \mathrm{J}_{A^{s}}$ is equal to $\Gamma(\mathscr{W} \times \mathscr{V}, \mathscr{S} / \mathscr{J}) \simeq \mathrm{S}_{\mathscr{W}} \times \mathscr{V} / \mathrm{J}_{A_{u}^{s}}$ if we identify $w_{i} / w_{u}$ with $w_{i u}$. Let $\widetilde{\mathrm{S} / \mathrm{J}_{A^{s}}}$ be the associated sheaf on $\mathscr{P} \times \mathscr{V}$ having global sections $\left[\mathrm{S} / \mathrm{J}_{A^{s}}\right]_{0}$; then we obviously have

$$
\widetilde{\mathrm{S} / \mathrm{J}_{A^{s}}} \simeq \mathscr{S} / \mathscr{J} .
$$

Define

$$
\Gamma_{*}(\mathscr{S} / \mathscr{J}):=\bigoplus_{a \in \mathbb{Z}} \Gamma(\mathscr{P} \times \mathscr{V},(\mathscr{S} / \mathscr{J})(a))
$$

We want to use the following result applied to the graded module $\mathrm{S} / \mathrm{J}_{A^{s}}$.

Proposition 5.22 ([GD61, Proposition 2.1.3]). There is the following exact sequence of $\mathbb{Z}$-graded S-modules:

$$
0 \longrightarrow H_{(\underline{w})}^{0}\left(\mathrm{~S} / \mathrm{J}_{A^{s}}\right) \longrightarrow \mathrm{S} / \mathrm{J}_{A^{s}} \longrightarrow \Gamma_{*}(\mathscr{S} / \mathscr{J}) \longrightarrow H_{(\underline{w})}^{1}\left(\mathrm{~S} / \mathrm{J}_{A^{s}}\right) \longrightarrow 0
$$

and for each $i \geqslant 1$, there is the isomorphism

$$
\bigoplus_{a \in \mathbb{Z}} H^{i}(\mathscr{P} \times \mathscr{V},(\mathscr{S} / \mathscr{J})(a)) \simeq H_{(\underline{w})}^{i+1}\left(\mathrm{~S} / \mathrm{J}_{A^{s}}\right),
$$

where $(\underline{w})$ is the ideal in $\mathbb{C}\left[z, \lambda_{0}, \ldots, \lambda_{n}, w_{0}, \ldots, w_{n}\right]$ generated by $w_{0}, \ldots, w_{n}$.

Proof. In the category of $\mathbb{C}\left[z, \lambda_{0}, \ldots, \lambda_{n}, w_{0}, \ldots, w_{n}\right]$-modules, the statement follows from [GD61, Proposition 2.1.3]. The statement in the category of S-modules follows from the proof given there.

In order to compute the local cohomology of $S / J_{A^{s}}$ we introduce a variant of the Ishida complex (see, for example [BH98, Theorem 6.2.5]). Let $T:=\mathbb{C}\left[w_{0}, \ldots, w_{n}, z \partial_{\lambda_{0}}, \ldots, z \partial_{\lambda_{n}}\right] \subset S$ be a commutative subring, and let $\mathbb{C}\left[\mathbb{N} A^{s}\right]$ be the affine semigroup algebra of $A^{s}$, that is,

$$
\mathbb{C}\left[\mathbb{N} A^{s}\right]=\left\{y^{\underline{c}} \in \mathbb{C}\left[y_{0}^{ \pm}, \ldots, y_{n+1}^{ \pm}\right] \mid \underline{c} \in \mathbb{N} A^{s} \subset \mathbb{Z}^{d+2}\right\} .
$$

We have a map

$$
\Phi_{A^{s}}: T \longrightarrow \mathbb{C}\left[\mathbb{N} A^{s}\right], \quad w_{i} \longmapsto y^{\underline{a}_{i}^{s}}, \quad z \partial_{\lambda_{i}} \longmapsto y^{\underline{b}_{i}^{s}} .
$$

Notice that the kernel $K_{A^{s}}$ of $\Phi_{A^{s}}$ is equal to the ideal in $T$ generated by the elements $\square_{(k, l)}$, hence

$$
T / K_{A^{s}} \simeq \mathbb{C}\left[\mathbb{N} A^{s}\right] .
$$

Remark 5.23. The $\mathbb{Z}$-grading of $T$ by the degree of the $w_{i}$ induces a $\mathbb{Z}$-grading on $\mathbb{C}\left[\mathbb{N} A^{s}\right]$ since the operators $\square_{(k, l)}$ are homogeneous. The semigroup ring $\mathbb{C}\left[\mathbb{N} A^{s}\right] \subset \mathbb{C}\left[\mathbb{Z}^{d+2}\right]$ also carries a natural $\mathbb{Z}^{d+2}$-grading. Looking at the matrix $A^{s}$, one sees that the $\mathbb{Z}$-grading coming from $T$ is the first component of this $\mathbb{Z}^{d+2}$-grading. 


\section{Hypergeometric Hodge modules}

We regard $\mathbb{C}\left[\mathbb{N} A^{s}\right]$ as a $T$-module using the map $\Phi_{A^{s}}$, which gives the isomorphisms

$$
S / J_{A^{s}} \simeq S \otimes_{T} T / K_{A^{s}} \simeq S \otimes_{T} \mathbb{C}\left[\mathbb{N} A^{s}\right] .
$$

We want to express the local cohomology of $S / J_{A^{s}}$ by the local cohomology of the commutative ring $\mathbb{C}\left[\mathbb{N} A^{s}\right]$. For this, let $I$ be the ideal in $\mathbb{C}\left[\mathbb{N} A^{s}\right]$ generated by $y^{a_{0}^{s}}, \ldots, y^{a_{n}^{s}}$; then we have the following change of rings formula.

Lemma 5.24. There is the following isomorphism of $\mathbb{Z}$-graded $S$-modules:

$$
H_{(\underline{w})}^{k}\left(S / J_{A^{s}}\right) \simeq S \otimes_{T} H_{I}^{k}\left(\mathbb{C}\left[\mathbb{N} A^{s}\right]\right) .
$$

Proof. Notice that if $S$ was commutative, this would be a standard property of the local cohomology groups. Here we have to adapt the proof slightly. First, notice that it is enough to compute $H_{(\underline{w})}^{k}\left(S / J_{A^{s}}\right)$ with an injective resolution of $T$-modules. To see why, let $I^{\bullet}$ be an injective resolution (in the category of $S$-modules) of $S / J_{A^{s}}$. Since $S$ is a free, hence flat, $T$-module, it follows from $\operatorname{Hom}_{S}\left(S \otimes_{T} M, I\right) \simeq S \otimes_{T} \operatorname{Hom}_{T}(M, I)$ that an injective $S$-module is also an injective $T$-module. Therefore, we have

$$
H_{(\underline{w})}^{k}\left(S / J_{A^{s}}\right) \simeq H^{k} \Gamma_{(\underline{w})}\left(I^{\bullet}\right)=H^{k} \Gamma_{I^{\prime}}\left(I^{\bullet}\right) \simeq H_{I^{\prime}}^{k}\left(S / J_{A^{s}}\right),
$$

where $I^{\prime}$ is the ideal in $T$ generated by $w_{0}, \ldots, w_{n}$ and the second isomorphism follows from the equality

$$
\Gamma_{(\underline{w})}\left(I^{k}\right)=\left\{x \in I^{k} \mid \forall i \exists k_{i} \text { such that } w_{i}^{k_{i}} x=0\right\}=\Gamma_{I^{\prime}}\left(I^{k}\right) .
$$

Let $J^{\bullet}$ be an injective resolution of $T / K_{A^{s}}$. In order to show the claim, consider the following isomorphisms:

$$
\begin{aligned}
S \otimes_{T} H_{I}^{K}\left(\mathbb{C}\left[\mathbb{N} A^{s}\right]\right) & \simeq S \otimes_{T} H_{I^{\prime}}^{k}\left(T / K_{A^{s}}\right) \simeq S \otimes_{T} H^{k} \Gamma_{I^{\prime}}\left(J^{\bullet}\right) \\
& \simeq H^{k}\left(S \otimes_{T} \Gamma_{I^{\prime}}\left(J^{\bullet}\right)\right) \simeq H^{k} \Gamma_{I^{\prime}}\left(S \otimes_{T} J^{\bullet}\right) \simeq H_{I^{\prime}}^{k}\left(S / J_{A^{s}}\right),
\end{aligned}
$$

where the third isomorphism follows from the fact that $S$ is a flat $T$-module and the fifth isomorphism follows from the fact that $S \otimes_{T} J^{\bullet}$ is a $T$-injective resolution of $S / J_{A^{s}} \simeq S \otimes_{T}$ $T / K_{A^{s}}$.

\subsection{Local cohomology of semigroup rings}

In this subsection, we compute local cohomology groups of some special semigroup rings associated with the semigroups $\mathbb{N} A^{s}$. These local cohomology groups turned up in Lemma 5.24. We will use their vanishing in Subsection 5.9 (specifically in Corollary 5.32) to prove the $\Gamma$-acyclicity of a Koszul complex. We show that these local cohomology groups can be expressed as the cohomology of the Ishida complex of $\mathbb{C}\left[\mathbb{N} A^{s}\right]$ (cf. Proposition 5.25). Since $A^{s}$ is a $(d+2) \times(2 n+2)$ integer matrix, the Ishida complex carries a natural $\mathbb{Z}^{d+2}$-grading. The vanishing of certain graded pieces of the Ishida complex and of its cohomology depends on the position of the degree as seen as an element in $\mathbb{R}^{d+2}$ in relation with the cone spanned by the columns of $A^{s}$ (cf. Lemma 5.26 and Proposition 5.30).

Let $\mathcal{F}$ be the face lattice of $\mathbb{R}_{\geqslant 0} A^{s}$, and denote by $\mathcal{F}_{\sigma}$ the sublattice of faces which lie in the face $\sigma$ spanned by $\underline{a}_{0}^{s}, \ldots, \underline{a}_{n}^{s}$. For a face $\sigma$ of $\mathbb{R}_{\geqslant 0} A^{s}$, consider the multiplicatively closed set

$$
U_{\sigma}:=\left\{y^{\underline{c}} \mid \underline{c} \in \mathbb{N}\left(A^{s} \cap \sigma\right)\right\},
$$

and denote the localization with respect to $U_{\sigma}$ by $\mathbb{C}\left[\mathbb{N} A^{s}\right]_{\sigma}=\mathbb{C}\left[\mathbb{N} A^{s}+\mathbb{Z}\left(A^{s} \cap \sigma\right)\right]$. We put

$$
L_{\sigma}^{k}=\bigoplus_{\substack{\tau \in \mathcal{F}_{\sigma} \\ \operatorname{dim} \tau=k}} \mathbb{C}\left[\mathbb{N} A^{s}\right]_{\tau}
$$




\section{T. Reichelt And C. Sevenheck}

and define maps $f^{k}: L_{\sigma}^{k} \rightarrow L_{\sigma}^{k+1}$ by specifying their components

$$
f_{\tau^{\prime}, \tau}^{k}: \mathbb{C}\left[\mathbb{N} A^{s}\right]_{\tau^{\prime}} \longrightarrow \mathbb{C}\left[\mathbb{N} A^{s}\right]_{\tau} \text { to be } \begin{cases}0 & \text { if } \tau^{\prime} \not \subset \tau, \\ \epsilon\left(\tau^{\prime}, \tau\right) \text { nat } & \text { if } \tau^{\prime} \subset \tau,\end{cases}
$$

where $\epsilon$ is a suitable incidence function on $\mathcal{F}_{\sigma}$ and nat is the natural localization morphism. The Ishida complex with respect to the face $\sigma$ is

$$
L_{\sigma}^{\bullet}: 0 \longrightarrow L_{\sigma}^{0} \longrightarrow L_{\sigma}^{1} \longrightarrow \cdots \longrightarrow L_{\sigma}^{d+1} \longrightarrow 0 .
$$

The Ishida complex with respect to the face $\sigma$ can be used to calculate local cohomology groups of $\mathbb{C}\left[\mathbb{N} A^{s}\right]$.

Proposition 5.25. As above, denote by $I \subset \mathbb{C}\left[\mathbb{N} A^{s}\right]$ the ideal generated by the elements $\Phi_{A^{s}}\left(w_{i}\right)=y^{a_{i}^{s}}$. Then for all $k$, we have the isomorphism

$$
H_{I}^{k}\left(\mathbb{C}\left[\mathbb{N} A^{s}\right]\right) \simeq H^{k}\left(L_{\sigma}^{\bullet}\right) .
$$

Proof. The proof can be easily adapted from [BH98, Theorem 6.2.5]. For the convenience of the reader, we sketch it here together with the necessary modifications . In order to show the claim, we have to prove that the functors $N \mapsto H^{k}\left(L_{\sigma}^{\bullet} \otimes N\right)$ form a universal $\delta$-functor (see, for example, [Har77]). If we can additionally show that

$$
H_{I}^{0}\left(\mathbb{C}\left[\mathbb{N} A^{s}\right]\right) \simeq H^{0}\left(L_{\sigma}^{\bullet}\right),
$$

the claim follows by [Har77, Corollary III.1.4]. Let $\mathcal{F}_{\sigma}(1)$ be the set of 1-dimensional faces in $\mathcal{F}_{\sigma}$, and notice that

$$
H_{I^{\prime}}^{0}\left(\mathbb{C}\left[\mathbb{N} A^{s}\right]\right) \simeq \operatorname{ker}\left(\mathbb{C}\left[\mathbb{N} A^{s}\right] \longrightarrow \bigoplus_{\tau \in \mathcal{F}_{\sigma}(1)} \mathbb{C}\left[\mathbb{N} A^{s}\right]_{\tau}\right) \simeq H^{0}\left(L_{\sigma}^{\bullet} \otimes_{T} M\right),
$$

where $I^{\prime} \subset \mathbb{C}\left[\mathbb{N} A^{s}\right]$ is the ideal generated by $\left\{y^{\underline{a}} \underline{a}_{i}^{s} \mid \mathbb{R}_{\geqslant 0} \underline{a}_{i}^{s} \in \mathcal{F}_{\sigma}(1)\right\}$. In order to show (5.15), we have to show that $\operatorname{rad} I^{\prime}=I$ since obviously $H_{I^{\prime}}^{0}\left(\mathbb{C}\left[\mathbb{N} A^{s}\right]\right)=H_{\operatorname{rad} I^{\prime}}^{0}\left(\mathbb{C}\left[\mathbb{N} A^{s}\right]\right)$. Since $I^{\prime} \subset I$ and $I=\operatorname{rad} I$ (here, $I$ is a prime ideal corresponding to the face spanned by $\underline{a}_{0}^{s}, \ldots, \underline{a}_{n}^{s}$ ), it is enough to check that a multiple of every $y^{\underline{c}} \in I$ lies in $I^{\prime}$. But this follows easily from the fact that the elements $\left\{\underline{a}_{i}^{s} \mid \mathbb{R}_{\geqslant 0} \underline{a}_{i}^{s} \in \mathcal{F}_{\sigma}(1)\right\}$ span the same cone over $\mathbb{Q}$ as the elements $\left\{\underline{a}_{0}^{s}, \ldots, \underline{a}_{n}^{s}\right\}$.

The proof that $N \mapsto H^{k}\left(L_{\sigma}^{\bullet} \otimes_{T} N\right)$ is a $\delta$-functor is completely parallel to the proof in [BH98].

Notice that the complex $L_{\sigma}^{\bullet}$ is $\mathbb{Z}^{d+2}$-graded since $\mathbb{C}\left[\mathbb{N} A^{s}\right]$ is $\mathbb{Z}^{d+2}$-graded. In order to analyze the cohomology of $L_{\sigma}^{\bullet}$, we look at its $\mathbb{Z}^{d+2}$-graded parts. For this, we have to determine when $\left(\mathbb{C}\left[\mathbb{N} A^{s}\right]_{\tau}\right)_{x} \neq 0\left(\right.$ and therefore $\left.\left(\mathbb{C}\left[\mathbb{N} A^{s}\right]_{\tau}\right)_{x} \simeq \mathbb{C}\right)$ for $x \in \mathbb{Z}^{d+2}$.

We are following [BH98, Chapter 6.3]. Denote the cone $\mathbb{R}_{\geqslant 0} A^{s} \subset \mathbb{R}^{d+2}$ by $C_{A^{s}}$. Let $x, y \in$ $\mathbb{R}^{d+2}$. We say that $y$ is visible from $x$ if $y \neq x$ and the line segment $[x, y]$ does not contain a point $y^{\prime} \in C_{A^{s}}$ with $y^{\prime} \neq y$. A subset $S$ is visible from $X$ if each $v \in S$ is visible from $x$.

Recall that the cone $C_{A^{s}}$ is given by the intersection of finitely many half-spaces

$$
H_{\tau}^{+}:=\left\{x \in \mathbb{R}^{d+2} \mid\left\langle n_{\tau}, x\right\rangle \geqslant 0\right\}, \quad \tau \in \mathcal{F}(d+1),
$$

where $\mathcal{F}(d+1)$ is the set of $(d+1)$-dimensional faces (facets) of $C_{A^{s}}$. We set

$$
x^{0}=\left\{\tau \mid\left\langle n_{\tau}, x\right\rangle=0\right\}, \quad x^{+}=\left\{\tau \mid\left\langle n_{\tau}, x\right\rangle>0\right\}, \quad x^{-}=\left\{\tau \mid\left\langle n_{\tau}, x\right\rangle<0\right\} .
$$

Lemma 5.26 ([BH98, Lemmas 6.3.2 and 6.3.3]). (i) A point $y \in C_{A^{s}}$ is visible from a point $x \in \mathbb{R}^{d+2} \backslash C_{A^{s}}$ if and only if $y^{0} \cap x^{-} \neq \emptyset$. 


\section{Hypergeometric Hodge modules}

(ii) Let $x \in \mathbb{Z}^{d+2}$, and let $\tau$ be a face of $C_{A^{s}}$. The $\mathbb{C}$-vector space $\left(\mathbb{C}\left[\mathbb{N} A^{s}\right]_{\tau}\right)_{x}$ is non-zero if and only if $\tau$ is not visible from $x$.

Recall that the facet $\sigma \in \mathcal{F}(d+1)$ is spanned by $\underline{a}_{0}^{s}, \ldots, \underline{a}_{n}^{s}$. It is the unique maximal element in the face lattice $\mathcal{F}_{\sigma} \subset \mathcal{F}$. Denote by $H_{\sigma}$ its supporting hyperplane (that is, $\sigma=C_{A^{s}} \cap H_{\sigma}$ ), which is given by

$$
H_{\sigma}=\left\{x \in \mathbb{R}^{d+2} \mid\left\langle n_{\sigma}, x\right\rangle=0\right\},
$$

where $n_{\sigma}=(0,1,0, \ldots, 0)$. Let $\tau \in \mathcal{F}_{\sigma}$ be a $k$-dimensional face contained in $\sigma$, and set $I_{\tau}:=\{i \mid$ $\left.\underline{a}_{i}^{s} \in \tau\right\}$. Notice that the vectors $\left\{\underline{a}_{i}^{s} \mid i \in I_{\tau}\right\}$ span the face $\tau$. This face $\tau$ gives rise to two other faces, namely its "shadow" $\tau^{s}$, which is spanned by the vectors $\left\{\underline{b}_{i}^{s} \mid \underline{a}_{i}^{s} \in \tau\right\}$, and the unique $(k+1)$-dimensional face $\tau^{c}$ which contains both $\tau$ and $\tau^{s}$. Let $\left\{\tau_{1}, \ldots, \tau_{m}\right\}=\mathcal{F}_{\sigma}(d)$ be the faces of dimension $d$ contained in $\sigma$, which give rise to the facets $\tau_{1}^{c}, \ldots, \tau_{m}^{c}$.

Example 5.27. Consider the matrix

$$
A^{s}=\left(\begin{array}{rrrrrr}
1 & 1 & 1 & 0 & 0 & 0 \\
0 & 0 & 0 & 1 & 1 & 1 \\
0 & 1 & -1 & 0 & 1 & -1
\end{array}\right) .
$$

The face $\sigma$ is generated by $(1,0,0),(1,0,1),(1,0,-1)$ and its shadow $\sigma^{s}$ is generated by $(0,1,0)$, $(0,1,1),(0,1,-1)$. The facet $\tau^{c}$ is generated by $\tau$ and its shadow $\tau^{s}$. See Figure 2.

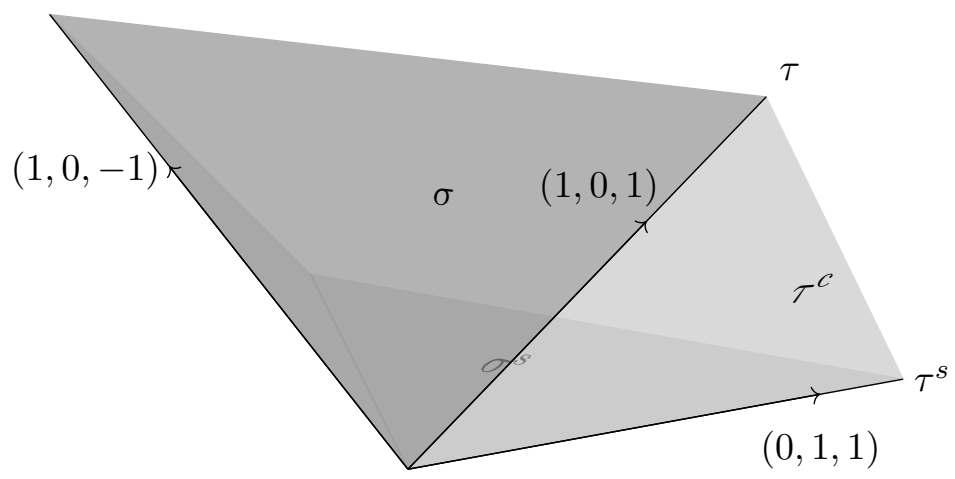

Figure 2. Facets corresponding to the matrix $A^{s}$ in Example 5.27

First, notice that by Lemma 5.26(i), the facet $\sigma$ is visible from a point $x \in \mathbb{R}^{d+2}$ if and only if $\left\langle n_{\sigma}, x\right\rangle<0$. If $\left\langle n_{\sigma}, x\right\rangle \geqslant 0$ holds, it follows from Lemma 5.26(i) that a face $\tau_{i} \subset \sigma$ is visible from $x$ if and only if the facet $\tau_{i}^{c}$ is visible from $x$; that is, $\left\langle n_{\tau_{i}^{c}}, x\right\rangle<0$.

We define

$$
S:=\mathbb{Z}^{d+2} \cap\left(\mathbb{R}\left(\underline{a}_{0}^{s}, \ldots, \underline{a}_{n}^{s}\right)+\mathbb{R}_{\geqslant 0}\left(\underline{b}_{0}^{s}, \ldots, \underline{b}_{n}^{s}\right)\right) ;
$$

this is the set of $\mathbb{Z}^{d+2}$-degrees occurring in $\mathbb{C}\left[\mathbb{N} A^{s}\right]_{\sigma}$. Also notice that we have

$$
H_{\sigma}=\mathbb{R}\left(\underline{a}_{0}^{s}, \ldots, \underline{a}_{n}^{s}\right) \quad \text { and } \quad H_{\sigma}^{+}=\mathbb{R}\left(\underline{a}_{0}^{s}, \ldots, \underline{a}_{n}^{s}\right)+\mathbb{R}_{\geqslant 0}\left(\underline{b}_{0}^{s}, \ldots, \underline{b}_{n}^{s}\right) .
$$

Given a point $x \in S$ with $\left\langle n_{\sigma}, x\right\rangle \geqslant 0$, we will construct a point $y_{x} \in \mathbb{Z}^{d+2}$ which lies in $H_{\sigma}$ such that $\tau_{i}$ is visible from $x$ if and only if it is visible from $y_{x}$ for all $i=1, \ldots, m$. Denote 


\section{T. Reichelt And C. Sevenheck}

by $z_{x}$ the projection of $x$ to the sub-vector space generated by $\underline{b}_{0}^{s}, \ldots, \underline{b}_{n}^{s}$. Since the semigroup generated by these vectors is saturated, we can express $z_{x}$ as a linear combination with positive integers

$$
z_{x}=\sum_{i=0}^{n} r_{i}^{x} \underline{b}_{i}^{s} \quad \text { with } \quad r_{i}^{x} \in \mathbb{N} .
$$

Since we have $0=\left\langle n_{\tau_{i}^{c}}, \underline{a}_{j}^{s}-\underline{b}_{j}^{s}\right\rangle=\left\langle n_{\tau_{i}^{c}},(1,-1,0, \ldots, 0)\right\rangle$ for any $\underline{a}_{j}^{s}, \underline{b}_{j}^{s} \in \tau_{i}^{c}$, the first two components of the vector $n_{\tau_{i}^{c}}$ are equal. Hence, if we set

$$
y_{x}:=x+\sum_{j=0}^{n} r_{j}^{x} \underline{a}_{j}^{s}-\sum_{i=0}^{n} r_{j}^{x} \underline{b}_{j}^{s},
$$

we easily see that

$$
\left\langle n_{\tau_{i}^{c}}, x\right\rangle=\left\langle n_{\tau_{i}^{c}}, y_{x}\right\rangle \text { for } i=1, \ldots, m .
$$

It follows that $\tau_{i}$ is visible from any point $x \in S$ if and only if it is visible from $y_{x}$, as required. Let us remark that the vectors $x$ and $y_{x}$ differ only in the first two components because the same is true for the pair of vectors $\left(\underline{a}_{i}^{s}, \underline{b}_{i}^{s}\right)$ for all $i \in\{0, \ldots, n\}$.

Lemma 5.28. In the above situation, let $x \in S$. Then $y_{x} \in S \cap H_{\sigma}$, and we have

$$
\left(L_{\sigma}^{\bullet}\right)_{x}=\left(L_{\sigma}^{\bullet}\right)_{y_{x}} .
$$

Proof. For the first point, notice that the vector $x-z_{x}$ is precisely the projection of $x$ to $H_{\sigma}$. On the other hand, we have $y_{x}=x-z_{x}+\sum_{i=0}^{n} n_{i}^{x} \underline{a}_{i}^{s}$, and $\sum_{i=0}^{n} n_{i}^{x} \underline{a}_{i}^{s}$ is an element of $H_{\sigma}$ anyhow.

The second statement is an easy consequence of Lemma 5.26(ii). More precisely, equation (5.16) shows that the visibility of some facet $\tau_{i}^{c}$ is the same from $x$ and from $y_{x}$. Moreover, $\sigma$ is not visible from both $x$ and $y_{x}$ (that is, $\left\langle n_{\sigma}, x\right\rangle \geqslant 0$ and $\left\langle n_{\sigma}, y_{x}\right\rangle \geqslant 0$ ); hence, also the visibility of $\tau_{i}$ is the same from $x$ and from $y_{x}$. We conclude that any localization $\mathbb{C}\left[\mathbb{N} A^{s}\right]_{\tau}$ (for any face $\tau \subset \sigma)$ vanishes in degree $x$ if and only if it vanishes in degree $y_{x}$. This yields the desired equality $\left(L_{\sigma}^{\bullet}\right)_{x}=\left(L_{\sigma}^{\bullet}\right)_{y_{x}}$.

We are now able to compute the cohomology of the Ishida complex with respect to the face $\sigma$. Set

and define

$$
H_{\tau_{i}^{c}}^{-}:=\left\{x \in \mathbb{R}^{d+2} \mid\left\langle n_{\tau_{i}^{c}}, x\right\rangle<0\right\} \quad \text { for } \quad i=1, \ldots, m
$$

$$
S^{-}:=\mathbb{Z}^{d+2} \cap H_{\sigma}^{+} \cap \bigcap_{i=1}^{m} H_{\tau_{i}^{c}}^{-} .
$$

Notice that $S=\mathbb{Z}^{d+2} \cap H_{\sigma}^{+}$; hence, we have a natural inclusion $S^{-} \subset S$.

Example 5.29. We consider again the matrix

$$
A^{s}=\left(\begin{array}{rrrrrr}
1 & 1 & 1 & 0 & 0 & 0 \\
0 & 0 & 0 & 1 & 1 & 1 \\
0 & 1 & -1 & 0 & 1 & -1
\end{array}\right)
$$

and take the point $x=(-1,1,1)$. Its projection to $\mathbb{R}\left(\underline{b}_{0}^{s}, \ldots, \underline{b}_{n}^{s}\right)$ is $(0,1,1)$; hence, we get

$$
y_{x}=x+(1,0,1)-(0,1,1)=(0,0,1) \in H^{\sigma} \text {. }
$$




\section{Hypergeometric Hodge modules}

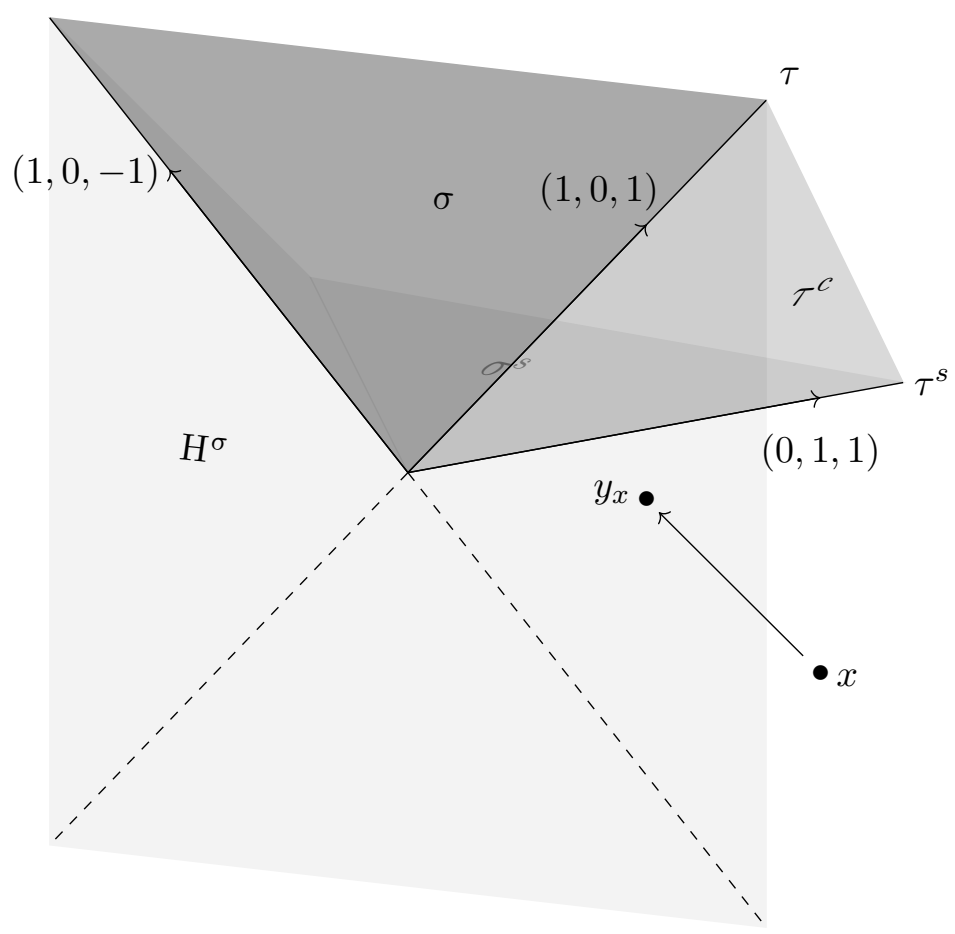

FiguRE 3. Facets corresponding to the matrix $A^{s}$ in Example 5.29

Proposition 5.30. Let $A^{s}$ be as above. Take any $x \in \mathbb{Z}^{d+2}$, and let $L_{\sigma}^{\bullet}$ be the Ishida complex with respect to the face $\sigma$ generated by $\underline{a}_{0}^{s}, \underline{a}_{1}^{s}, \ldots, \underline{a}_{n}^{s}$.

(i) If $x \notin S$, then $\left(L_{\sigma}^{\bullet}\right)_{x}=0$.

(ii) If $x \in S \backslash S^{-}$, then $H^{i}\left(L_{\sigma}^{\bullet}\right)_{x}=0$ for all $i$.

(iii) If $x \in S^{-}$, then $H^{i}\left(L_{\sigma}^{\bullet}\right)_{x}=0$ for $i \neq d+1$ and $H^{d+1}\left(L_{\sigma}^{\bullet}\right)_{x} \simeq \mathbb{C}$.

Proof. The first point follows from the fact that we have $\left(\mathbb{C}\left[\mathbb{N} A^{s}\right]_{\sigma}\right)_{x}=0$ for $x \notin S$, hence $\left(L_{\sigma}^{i}\right)_{x}=0$ for all $i$. For the proof of the second and third point, it is sufficient to consider the case where $x \in H_{\sigma}$ : Namely, in both cases, we have $x \in S$, so that Lemma 5.28 applies. We can thus replace $x$ by $y_{x}$, that is, $\left(L_{\sigma}^{\bullet}\right)_{x}=\left(L_{\sigma}^{\bullet}\right)_{y_{x}}$. Moreover, $x \in S^{-}$if and only if $y_{x} \in S^{-} \cap H_{\sigma}$ by formula (5.16). Hence, we will suppose in the remainder of this proof that $x \in S \cap H_{\sigma}$.

We will reduce statements (ii) and (iii) for $x \in S \cap H_{\sigma}$ to the computation of the local cohomology of a semigroup ring with respect to a maximal ideal via the Ishida complex, as done in [BH98, Theorem 6.3.4]. For this, we will use the matrix $\widetilde{A}=\left(\underline{\widetilde{a}}_{0}, \underline{\widetilde{a}}_{1}, \ldots, \underline{\widetilde{a}}_{n}\right)$, which can be seen as the matrix of the first $n+1$ columns of $A^{s}$, with the second row deleted. The semigroup $\mathbb{N} \widetilde{A}$ (respectively, the cone $C_{\widetilde{A}}$ ) embeds into $\mathbb{N} A^{s}$ (respectively, into $C_{A^{s}}$ ) via the map $\underline{\widetilde{a}}_{i} \mapsto \underline{a}_{i}^{s}$, and these embeddings are compatible with the embeddings $\mathbb{R}^{r+1} \hookrightarrow \mathbb{R}^{r+2}$ (respectively, $\mathbb{Z}^{r+1} \hookrightarrow \mathbb{Z}^{r+2}$ ) given by

$$
\left(x_{1}, x_{3}, x_{4} \ldots, x_{d+2}\right) \longmapsto\left(x_{1}, 0, x_{3}, x_{4}, \ldots, x_{d+2}\right) .
$$

The following equality of semigroups holds true:

$$
S^{-} \cap H_{\sigma}=\mathbb{Z}^{d+1} \cap \operatorname{Int}\left(-C_{\widetilde{A}}\right)
$$




\section{T. Reichelt And C. Sevenheck}

where both intersections are taken in $\mathbb{Z}^{d+2}$. To show this, notice that $C_{\widetilde{A}}=\mathbb{R}_{\geqslant 0}\left(\underline{a}_{0}^{s}, \ldots, \underline{a}_{n}^{s}\right) \cap H_{\sigma}$, that $\tau_{i}^{c} \cap H_{\sigma}=\tau_{i}$ and hence

$$
\bigcap_{i=1}^{m}\left(H_{\tau_{i}^{c}}^{-} \cap H_{\sigma}\right)=\operatorname{Int}\left(-C_{\widetilde{A}}\right) .
$$

Consider the projection map

$$
p: \mathbb{R}^{d+2} \longrightarrow \mathbb{R}^{d+1}, \quad\left(x_{1}, x_{2}, x_{3}, \ldots, x_{d+2}\right) \longmapsto\left(x_{1}, x_{3}, \ldots, x_{d+2}\right),
$$

which forgets the second component; then for all $\tau \subset \sigma$ and all elements $x \in S \cap H_{\sigma}$, we have

$$
\left(\mathbb{C}\left[\mathbb{N} A^{s}\right]_{\tau}\right)_{x} \simeq\left(\mathbb{C}[\mathbb{N} \widetilde{A}]_{p(\tau)}\right)_{p(x)} .
$$

Under this isomorphism, the $\mathbb{Z}^{d+2}$-graded part $\left(L_{\sigma}^{\bullet}\right)_{x}$ of the Ishida complex with respect to the face $\sigma$ goes over to the $\mathbb{Z}^{d+1}$-graded part $\left(L^{\bullet}\right)_{p(x)}$ of the Ishida complex considered in [BH98] (that is, the Ishida complex of the semigroup $\mathbb{C}[\mathbb{N} \widetilde{A}]$ with respect to the maximal ideal generated by $\left.\left(w_{0}, \ldots, w_{n}\right)\right)$. By formula (5.17), the proposition now follows from [BH98, Theorem 6.3.4].

We finish this subsection with the following easy consequence, which will be crucial in the proof of the main result (Theorem 5.35 below).

Corollary 5.31. In the above situation, we have $H^{i}\left(L_{\sigma}^{\bullet}\right)=0$ for all $i \neq d+1, H^{d+1}\left(L_{\sigma}^{\bullet}\right)_{x}=0$ for all $x \in \mathbb{Z}^{d+2} \backslash S^{-}$and $\operatorname{deg}_{\mathbb{Z}}\left(H^{d+1}\left(L_{\sigma}^{\bullet}\right)_{x}\right)<0$ for $x \in S^{-}$, where $\operatorname{deg}_{\mathbb{Z}}(-)$ refers to the $\mathbb{Z}$-grading of $H^{i}\left(L_{\sigma}^{\bullet}\right)$ corresponding to the first row of $A^{s}$.

In other words, the cohomology groups of the Ishida complex (with respect to the face $\sigma$ ) are concentrated in negative degrees.

Proof. The first two statements are precisely those from Proposition 5.30, items (i) and (ii). In order to show the third one, notice that for any $x \in S$, we have $\operatorname{deg}_{\mathbb{Z}}(x) \leqslant \operatorname{deg}_{\mathbb{Z}}\left(y_{x}\right.$ ) (this follows from the very definition of the vector $\left.y_{x}\right)$. Now, let $x \in S^{-}$, and suppose $H^{d+1}\left(L_{\sigma}^{\bullet}\right)_{x} \neq 0$. From Lemma 5.28, we deduce that

$$
H^{d+1}\left(L_{\sigma}^{\bullet}\right)_{x}=H^{d+1}\left(L_{\sigma}^{\bullet}\right)_{y_{x}}
$$

and as already remarked above, $y_{x} \in S^{-} \cap H_{\sigma}$ because $x \in S^{-}$. However, we deduce from formula (5.17) that $\operatorname{deg}_{\mathbb{Z}}\left(y_{x}\right)<0$ if $y_{x} \in S^{-} \cap H_{\sigma}$, so that we obtain $\operatorname{deg}_{\mathbb{Z}}\left(H^{d+1}\left(L_{\sigma}^{\bullet}\right)_{x}\right)<0$, as required.

\subsection{Statement and proof of the main theorem}

In this subsection, we finally finish the computation of the direct image $\pi_{2+} \mathscr{N}$. The $\Gamma$-cyclicity of $\mathscr{S} / \mathscr{J}$ (cf. Corollary 5.32), which follows directly from the results of Subsections 5.7 and 5.8, enables us to compute the global sections of $\pi_{2+} \mathscr{N}$ as the cohomology of a Koszul complex (cf. Proposition 5.33). Finally, we are able to compute the Hodge filtration on the GKZ system in Theorem 5.35.

Corollary 5.32. The $\mathscr{S}$-modules $\mathscr{S} / \mathscr{J}$ are $\Gamma$-acyclic.

Proof. If we consider the degree zero part of formula (5.14), then it suffices to show that the $\mathbb{Z}$-graded local cohomology $S$-modules $H_{(w)}^{*}\left(S / J_{A_{s}}\right)$ are concentrated in negative degrees. By Proposition 5.25 and Lemma 5.24, these local cohomology groups are calculate by the Ishida complex $L_{\sigma}^{\bullet}$; that is, we have isomorphisms

$$
H_{(w)}^{k}\left(S / J_{A_{s}}\right) \simeq S \otimes_{T} H_{I}^{k}\left(\mathbb{C}\left[\mathbb{N} A^{s}\right]\right) \simeq S \otimes_{T} H^{k}\left(L_{\sigma}^{\bullet}\right)
$$




\section{Hypergeometric Hodge modules}

The cohomology groups $H^{k}\left(L_{\sigma}^{\bullet}\right)$ are concentrated in negative degrees by Corollary 5.31 (and tensoring with $S$ does not change the $\mathbb{Z}$-degree, which is counted with respect to the degree of the variables $\left.w_{0}, \ldots, w_{n}\right)$. Hence, the result follows.

Proposition 5.33. There is the following isomorphism in $D^{b}\left(\mathscr{R}_{V}\right)$ :

$$
\Gamma \pi_{2+} \mathscr{N} \simeq \Gamma\left(\operatorname{Kos}^{\bullet}\left(z^{-d} \mathscr{S} / \mathscr{J},\left(\widetilde{E}-\beta_{k}\right)_{k=0, \ldots, d}\right)\right) .
$$

Proof. By formula (5.11) and Propositions 5.20 and 5.21, we have the isomorphisms

$$
\begin{aligned}
\Gamma \pi_{2+\mathscr{N}} & \simeq \Gamma R \pi_{2 *}\left(\Omega_{\mathscr{P} \times \mathscr{V} / \mathscr{V}}^{\bullet+n} \otimes \mathscr{N}\right) \simeq R \Gamma R \pi_{2 *}\left(\Omega_{\mathscr{P} \times \mathscr{V} / \mathscr{V}}^{\bullet+n} \otimes \mathscr{N}\right) \\
& \simeq R \Gamma\left(\Omega_{\mathscr{P} \times \mathscr{V} / \mathscr{V}}^{\bullet+n} \otimes \mathscr{N}\right) \simeq R \Gamma\left(\mathscr{L}^{\bullet}\right) .
\end{aligned}
$$

Using the last isomorphism in (5.13) and Corollary 5.32, we get

$$
R \Gamma\left(\mathscr{L}^{\bullet}\right) \simeq R \Gamma\left(\operatorname{Kos}^{\bullet}\left(z^{-d} \mathscr{S} / \mathscr{J},(\tilde{E})_{k=0, \ldots, d}\right)\right) \simeq \Gamma\left(\operatorname{Kos}^{\bullet}\left(z^{-d} \mathscr{S} / \mathscr{J},(\tilde{E})_{k=0, \ldots, d}\right)\right) .
$$

Denote by $\mathrm{R}_{\mathscr{V}}$ the ring

$$
\mathrm{R}_{\mathscr{V}}=\mathbb{C}\left[z, \lambda_{0}, \ldots, \lambda_{n}\right]\left\langle z \partial_{\lambda_{0}}, \ldots, z \partial_{\lambda_{n}}\right\rangle,
$$

let $J_{\widetilde{A}}^{\lambda} \subset \mathrm{R}_{\mathscr{V}}$ be the left ideal generated by

$$
\square_{\underline{l}}^{\lambda}=\prod_{l_{i}>0}\left(z \partial_{\lambda_{i}}\right)^{l_{i}}-\prod_{l_{i}<0}\left(z \partial_{\lambda_{i}}\right)^{-l_{i}} \quad \text { for } \underline{l} \in \mathbb{L}_{\widetilde{A}},
$$

and let $I_{\widetilde{A}}^{\lambda} \subset \mathrm{R}_{\mathscr{V}}$ be the left ideal generated by $J_{\widetilde{A}}^{\lambda}$ and the operators

$$
\tilde{E}_{k}-\beta_{k}:=\sum_{i=1}^{n} a_{k i} \lambda_{i} z \partial_{\lambda_{i}}-\beta_{k} \quad \text { for } k=1, \ldots, d, \quad \tilde{E}_{0}-\beta_{0}:=\sum_{i=0}^{n} \lambda_{i} z \partial_{\lambda_{i}}-\beta_{0} .
$$

LEMma 5.34. There is the following isomorphism of $\mathrm{R}_{\mathscr{V}}$-modules:

$$
\Gamma \mathcal{H}^{0}\left(\pi_{2+} \mathscr{N}\right) \simeq \mathcal{H}^{0}\left(\Gamma \pi_{2+} \mathscr{N}\right) \simeq z^{-d} \mathrm{R}_{\mathscr{V}} / I_{\widetilde{A}}^{\lambda}
$$

Proof. The first isomorphism follows from Lemma 5.16. The second isomorphism follows from Proposition 5.33, the isomorphism $\Gamma(\mathscr{S} / \mathscr{J}) \simeq \mathrm{R}_{\mathscr{V}} / J_{\widetilde{A}}^{\lambda}$ and the isomorphism

$$
z^{-d} \mathrm{R}_{\mathscr{V}} / I_{\widetilde{A}}^{\lambda} \simeq H^{0}\left(\operatorname{Kos}^{\bullet}\left(z^{-d} \mathrm{R}_{\mathscr{V}} / J_{\widetilde{A}}^{\lambda},\left(\tilde{E}_{k}-\beta_{k}\right)_{k=0, \ldots, d}\right)\right) .
$$

We are now able to prove the main theorem of this paper. Let $\widetilde{A}$ be the $(d+1) \times(n+1)$ integer matrix

$$
\widetilde{A}=\left(\begin{array}{cccc}
1 & 1 & \ldots & 1 \\
0 & a_{11} & \ldots & a_{1 n} \\
\vdots & \vdots & & \vdots \\
0 & a_{d 1} & \ldots & a_{d n}
\end{array}\right)
$$

given by a matrix $A=\left(a_{j k}\right)$ such that $\mathbb{Z} \widetilde{A}=\mathbb{Z}^{d+1}$ and such that $\mathbb{N} \widetilde{A}=\mathbb{Z}^{d+1} \cap \mathbb{R}_{\geqslant 0} \widetilde{A}$.

Theorem 5.35. Let $\widetilde{A}$ be an integer matrix as above, $\widetilde{\beta} \in \mathfrak{A}_{\widetilde{A}}$ and $\beta_{0} \in(-1,0]$. The GKZ system $\mathcal{M}_{\widetilde{A}}^{\widetilde{\beta}}$ carries the structure of a mixed Hodge module whose Hodge filtration is given by the shifted order filtration, that is,

$$
\left(\mathcal{M}_{\widetilde{A}}^{\widetilde{\beta}}, F_{\bullet}^{H}\right) \simeq\left(\mathcal{M}_{\widetilde{A}}^{\widetilde{\beta}}, F_{\bullet+d}^{\text {ord }}\right) .
$$




\section{T. Reichelt And C. Sevenheck}

Proof. Recall from Proposition 5.4 that we have the isomorphism

$$
H_{\mathcal{M}}^{\widetilde{\beta}} \simeq \mathcal{H}^{2 n+d+1}\left(p_{*}\left(q^{* p} \mathbb{C}_{T}^{\beta} \otimes F^{*} j_{!}^{p} \mathbb{C}_{\mathbb{C}^{*}}^{-\beta_{0}-1}\right)\right) \in \operatorname{MHM}(V) .
$$

The underlying $\mathcal{D}_{V}$-module of this mixed Hodge module is

$$
\mathcal{H}^{2 n+d+1}\left(p_{+}\left(q^{\dagger} \mathcal{O}_{T}^{\beta} \otimes_{\mathcal{O}} F^{\dagger}\left(j_{\dagger} \mathcal{O}_{\mathbb{C}^{*}}^{-\beta_{0}-1}\right)\right)\right) \simeq \mathcal{H}^{0}\left(\pi_{2+} \mathcal{N}\right) .
$$

We have already computed the Hodge filtration of $\mathcal{N}$ (more precisely, we have computed it on the restrictions $\mathcal{N}_{u}$ of $\mathcal{N}$ to each chart $W_{u} \times V$ in Proposition 5.11). In order to compute the Hodge filtration under the direct image of $\pi_{2}$, we will use the results obtained above and read off the Hodge filtration from the corresponding $\mathscr{R}_{V}$-module $\mathscr{T}\left(\mathcal{M}_{\widetilde{A}}^{\widetilde{\beta}}, F^{H}\right)$. We have the following isomorphisms:

$$
\Gamma \mathscr{T}\left(\mathcal{M}_{\widetilde{A}}^{\widetilde{\beta}}, F^{H}\right) \simeq \Gamma \mathscr{T}\left(\mathcal{H}^{0}\left(\pi_{2+} \mathcal{N}, F^{H}\right)\right) \simeq \Gamma \mathcal{H}^{0}\left(\pi_{2+} \mathscr{N}\right) \simeq z^{-d} \mathrm{R}_{\mathscr{V}} / I_{\widetilde{A}}^{\lambda} .
$$

Using these isomorphisms, the claim follows easily.

\subsection{Duality}

For applications like the one presented in the next section, it will be useful to extend the computation of the Hodge filtration on $\mathcal{M}_{\widetilde{A}}^{\beta}$ to the dual Hodge module $\mathbb{D} \mathcal{M}_{\widetilde{A}}^{\beta}$. This is possible under the assumption made in the main theorem, Theorem 5.35, plus the extra requirement that the semigroup ring $\mathbb{C}[\mathbb{N} \widetilde{A}]$ is Gorenstein. More precisely, it follows from [Wal07] that under these assumptions, the $\mathcal{D}_{V}$-module $\mathbb{D} \mathcal{M}_{\widetilde{A}}^{\beta}$ is still a GKZ system. Hence, it is reasonable to expect that its Hodge filtration will also be the order filtration up to a suitable shift.

The Gorenstein condition for normal semigroup rings has a well-known combinatorial expression (see [BH98, Corollary 6.3.8]), namely, $\mathbb{C}[\mathbb{N} \widetilde{A}]$ is Gorenstein if and only if there is a vector $\widetilde{c}$ such that the set of interior points $\operatorname{int}(\mathbb{N} \widetilde{A})$ (that is, the intersection $\operatorname{int}\left(\mathbb{R}_{\geqslant 0} A\right) \cap \mathbb{Z}^{d+1}$ ) is given by $\widetilde{c}+\mathbb{N} \widetilde{A}$.

Theorem 5.36. Suppose that $\widetilde{A} \in M(d+1 \times n+1, \mathbb{Z})$ is such that $\mathbb{Z} \widetilde{A}=\mathbb{Z}^{d+1}$ and $\mathbb{N} \widetilde{A}=$ $\mathbb{Z}^{d+1} \cap \mathbb{R}_{\geqslant 0} \widetilde{A}$ and such that $\operatorname{int}(\mathbb{N} \widetilde{A})=\widetilde{c}+\mathbb{N} \widetilde{A}$ for some $\widetilde{c}=\left(c_{0}, c\right) \in \mathbb{Z}^{d+1}$, and take $\widetilde{\beta} \in \mathfrak{A}_{A}$. Then we have

$$
\mathbb{D} \mathcal{M}_{\widetilde{A}}^{\widetilde{\beta}} \simeq \mathcal{M}_{\widetilde{A}}^{-\widetilde{\beta}-\widetilde{c}}
$$

and the Hodge filtration on $\mathbb{D} \mathcal{M}_{\widetilde{A}}^{\widetilde{\beta}}$ is the order filtration shifted by $n+c_{0}$; that is, we have

$$
F_{p}^{H} \mathbb{D} \mathcal{M}_{\widetilde{A}}^{\widetilde{\beta}} \simeq F_{p-n-c_{0}}^{\text {ord }} \mathcal{M}_{\widetilde{A}}^{-\widetilde{\beta}-\widetilde{c}} .
$$

Proof. The proof is very much parallel to those of [RS15, Proposition 2.19] and [RS17, Theorem 5.4]; we will give the main ideas here once again for the convenience of the reader. We again work with the modules of global sections and write $D_{V}:=\mathbb{C}\left[\lambda_{0}, \ldots, \lambda_{n}\right]\left\langle\partial_{\lambda_{0}}, \ldots, \partial_{\lambda_{n}}\right\rangle$ and $S_{\widetilde{A}}$ for the commutative ring $\mathbb{C}\left[\partial_{\lambda_{0}}, \ldots, \partial_{\lambda_{n}}\right] /\left(\square_{\underline{l}}\right)_{\underline{l} \in \mathbb{L}_{\widetilde{A}}}$ These rings are $\mathbb{Z}^{d+1}$-graded by $\operatorname{deg}\left(\lambda_{i}\right)=-\widetilde{a}_{i}$ and $\operatorname{deg}\left(\partial_{\lambda_{i}}\right)=\widetilde{a}_{i}$.

In order to calculate $\mathbb{D} M_{\widetilde{A}}^{\widetilde{A}}$ together with its Hodge filtration, we need to find a strictly filtered free resolution $\left(L_{\bullet}, F_{\bullet}\right) \stackrel{\simeq}{\rightarrow}\left(M_{\widetilde{A}}^{\widetilde{\beta}}, F_{\bullet}^{H}\right)=\left(M_{\widetilde{A}}^{\widetilde{\beta}}, F_{\bullet+d}^{\text {ord }}\right)$. We have already used in the previous sections of this paper resolutions of "Koszul"-type for various (filtered) $\mathcal{D}$-modules. Here we consider the Euler-Koszul complex

$$
K^{\bullet}:=\operatorname{Kos}\left(D_{V} \otimes_{\mathbb{C}\left[\underline{\partial}_{\lambda}\right]} S_{\widetilde{A}},\left(E_{k}-\beta_{k}\right)_{k=0, \ldots, d}\right),
$$




\section{Hypergeometric Hodge modules}

as defined in Subsection 3.1, and a generalization to $\mathbb{Z}^{d+1}$-graded $\mathbb{C}\left[\underline{\partial}_{\lambda}\right]$-modules (for details, see [MMW05]).

A free resolution of $M_{\widetilde{A}}^{\widetilde{\beta}}$ is constructed as follows: Take a $\mathbb{C}[\underline{\partial}]$-free graded resolution of $T^{\bullet} \rightarrow S_{\widetilde{A}}$, and define $L^{\bullet}$ to be the total complex $\operatorname{Tot}\left(K^{\bullet}\left(E-\beta, D_{V} \otimes_{\mathbb{C}\left[\underline{\partial}_{\lambda}\right]} T^{\bullet}\right)\right)$. The double complex $K^{\bullet}\left(E-\beta, D_{V} \otimes_{\mathbb{C}\left[\underline{\partial}_{\lambda}\right]} T^{\bullet}\right)$ exists since $K^{\bullet}\left(E-\beta, D_{V} \otimes_{\mathbb{C}[\underline{\partial}]}-\right)$ is a functor from the category of $\mathbb{Z}^{d+1}$-graded $\mathbb{C}[\underline{\partial}]$-modules to the category of (bounded complexes of) $\mathbb{Z}^{d+1}$-graded $D_{V}$-modules. Then we have $L^{-k}=0$ for all $k>n+1$ (the length of the Euler-Koszul complexes is $d+1$, and the length of the resolution $T_{\bullet} \rightarrow P$ is $n-d+1$; hence, the total complex has length $(d+1)+(n-d+1)-1=n+1)$. Moreover, the last term $L^{-n-1}$ of this complex is simply equal to $D_{V}$ (and so is the first one, $L^{0}$ ).

As we have $\operatorname{int}(\mathbb{N} \widetilde{A})=\widetilde{c}+\mathbb{N} \widetilde{A}$, the $\operatorname{ring} \mathbb{C}[\mathbb{N} \widetilde{A}] \simeq S_{\widetilde{A}}$ is Gorenstein; more precisely, we have $\omega_{S_{\widetilde{A}}} \simeq S_{\widetilde{A}}(\widetilde{c})$, where $\omega_{S_{\widetilde{A}}}$ is the canonical module of $S_{\widetilde{A}}$. Then a spectral sequence argument (see also [Wal07, Proposition 4.1]), using

$$
\operatorname{Ext}_{\mathbb{C}[\underline{\partial}]}^{i}\left(S_{\widetilde{A}}, \omega_{\mathbb{C}[\underline{\partial}]}\right) \simeq \begin{cases}0 & \text { if } i<n-d, \\ S_{\widetilde{A}}(\widetilde{c}) & \text { if } i=n-d,\end{cases}
$$

shows that

$$
\mathbb{D} M_{\widetilde{A}}^{\widetilde{\beta}} \cong M_{\widetilde{A}}^{-\widetilde{\beta}-\widetilde{c}}
$$

In order to calculate the Hodge filtration on $M_{\widetilde{A}}^{-\widetilde{c}}$, we remark that the Euler-Koszul complex is naturally filtered by putting

$$
F_{p} K^{-l}:=\bigoplus_{0 \leqslant i_{1}<\cdots<i_{l} \leqslant l} F_{p+d-l}^{\mathrm{ord}}\left(D_{V} \otimes_{\mathbb{C}[\partial]} S_{\widetilde{A}}\right) e_{i_{1} \ldots i_{l}} .
$$

Notice that $D_{V} \otimes_{\mathbb{C}[\underline{\partial}]} S_{\widetilde{A}} \simeq D_{V} /\left(\square_{\underline{l}}\right)_{\underline{l} \in \mathbb{L}_{\tilde{A}}}$, so that this $D_{V}$-module has an order filtration induced from $F_{\bullet}^{\text {ord }} D_{V}$. In order to show that $\left(K^{\bullet}, F_{\bullet}\right) \rightarrow\left(M_{\widetilde{A}}^{\widetilde{\beta}}, F^{H}\right)$ is a filtered quasi-isomorphism, it suffices (by Lemma 3.2) to show that $\mathrm{Gr}_{\bullet}^{F} K^{\bullet} \rightarrow \mathrm{Gr}_{\bullet}^{F^{H}} M_{\widetilde{A}}^{\widetilde{\beta}}$ is a quasi-isomorphism. This follows from [SST00, formula (4.32) and Lemma 4.3.7], as $\mathbb{C}[\mathbb{N} \widetilde{A}]$ is Cohen-Macaulay due to the normality assumption on $\widetilde{A}$. The final step is to endow the free resolution $L^{\bullet}=\operatorname{Tot}\left(K^{\bullet}(E-\right.$ $\left.\left.\beta, D_{V} \otimes_{\mathbb{C}\left[\underline{\partial}_{\lambda}\right]} T^{\bullet}\right)\right)$ with a strict filtration $F_{\bullet}$ and to show that $\left(L_{\bullet}, F_{\bullet}\right) \stackrel{\simeq}{\rightarrow}\left(M_{\widetilde{A}}^{\widetilde{\beta}}, F_{\bullet}^{H}\right)$. As the resolution $T_{\bullet} \rightarrow S_{\widetilde{A}}$ is taken in the category of $\mathbb{Z}^{d+1}$-graded $\mathbb{C}[\underline{\partial}]$-modules, the morphisms of this resolution are homogenous for the $\left(\mathbb{Z}\right.$-)grading $\operatorname{deg}\left(\lambda_{i}\right)=-1$ and $\operatorname{deg}\left(\partial_{\lambda_{i}}\right)=1$ (notice that this is the grading given by the first component of the $\mathbb{Z}^{d+1}$-grading of the ring $\left.D_{V} \otimes_{\mathbb{C}[\underline{\partial}]} S_{\widetilde{A}}\right)$. Hence, these morphisms are naturally filtered for the order filtration $F_{\bullet}^{\text {ord }}\left(D_{V} \otimes_{\mathbb{C}[\underline{\partial}]} S_{\widetilde{A}}\right)$, and they are even strict: for a map given by homogenous operators from $\mathbb{C}[\partial]$, taking the symbols has simply no effect, so that $\operatorname{Gr}_{\bullet}^{F}\left(D_{V} \otimes_{\mathbb{C}[\underline{\partial}]} T_{\bullet}\right) \rightarrow \operatorname{Gr}_{\bullet}^{F^{\text {ord }}}\left(D_{V} \otimes_{\mathbb{C}[\underline{\partial}]} S_{\widetilde{A}}\right)$ is a filtered quasi-isomorphism (and similarly for the sums occurring in the terms $K^{-l}$ ). However, we have to determine the $\mathbb{Z}$-degree (for the grading $\operatorname{deg}\left(\partial_{\lambda_{i}}\right)=1$ ) of the highest (actually, the only non-zero) cohomology module $\operatorname{Ext}_{\mathbb{C}[\underline{\partial}]}^{n-d}\left(S_{\widetilde{A}}, \omega_{\mathbb{C}[\underline{\partial}]}\right):$ it is the first component of the difference of the degree of $\omega_{\mathbb{C}[\underline{\partial}]}$ (that is, the first component of the sum of the columns of $\widetilde{A}$ ), which is $n+1$, and the first component of the degree of $\omega_{S_{\widetilde{A}}}$, which is $c_{0}$. Now the shift of the filtration between $M_{\widetilde{A}}^{\widetilde{\beta}}$ and the dual module $M_{\widetilde{A}}^{-\widetilde{\beta}-\left(c_{0}, c\right)}$ is the sum of the length of the complex $K^{\bullet}\left(E-\beta, D_{V} \otimes_{\mathbb{C}[\underline{\partial]}} S_{\widetilde{A}}\right)$, that is, $d+1$, and 


\section{T. Reichelt And C. Sevenheck}

the above $\mathbb{Z}$-degree of $\operatorname{Ext}_{\mathbb{C}[\partial]}^{n-d}\left(S_{\widetilde{A}}, \omega_{\mathbb{C}[\partial]}\right)$, that is, $n+1-c_{0}$. Hence, the filtration $F_{\bullet} L^{-n-1}$ is again the shifted order filtration; more precisely, we have

$$
F_{p} L^{-n-1}=F_{p+d-(d+1)-\left(n+1-c_{0}\right)}^{\text {ord }} D_{V}=F_{p-n-2+c_{0}}^{\text {ord }} D_{V}
$$

Now, it follows from [Sai94, p. 55] that

$$
\mathbb{D}\left(M_{\widetilde{A}}^{\widetilde{\beta}}, F^{H}\right) \simeq \operatorname{Hom}_{D_{V}}\left(\left(L^{\bullet}, F_{\bullet}\right),\left(\left(D_{V} \otimes \Omega_{V}^{n+1}\right)^{\vee}, F_{\bullet-2(n+1)} D_{V} \otimes\left(\Omega_{V}^{n+1}\right)^{\vee}\right)\right),
$$

so that we finally obtain

$$
F_{p}^{H} \mathbb{D} M_{\widetilde{A}}^{\widetilde{\beta}}=F_{p-n-c_{0}}^{\text {ord }} M_{\widetilde{A}}^{-\widetilde{\beta}-\left(c_{0}, c\right)} .
$$

We now consider the special case $\beta=0$. From Proposition 5.2, we know that up to multiplication by a non-zero constant, we have the morphism

$$
\begin{gathered}
\phi: F_{p+d-c_{0}}^{\text {ord }} M_{\widetilde{A}}^{-\left(c_{0}, c\right)}=F_{p+n+d}^{H} \mathbb{D}\left(M_{\widetilde{A}}^{0}\right)=F_{p}^{H} \mathbb{D}\left(M_{\widetilde{A}}^{0}\right)(-n-d) \\
\longrightarrow F_{p}^{H} M_{\widetilde{A}}^{0}=F_{p+d}^{\text {ord }} M_{\widetilde{A}}^{0}, \\
P \longmapsto P \cdot \partial^{\left(c_{0}, c\right)},
\end{gathered}
$$

where $\partial^{\left(c_{0}, c\right)}:=\prod_{i=0}^{n} \partial_{\lambda_{i}}^{k_{i}}$ for any $\underline{k}=\left(k_{0}, \ldots, k_{n}\right)$ with $\widetilde{A} \cdot \underline{k}=\left(c_{0}, c\right)$. Since $\widetilde{A}$ is homogeneous, we have $\sum k_{i}=c_{0}$. As a consequence, we obtain the following result.

COROLlary 5.37. Under the above assumptions on $\widetilde{A}$, the morphism

$$
\phi:\left(M_{\widetilde{A}}^{-\left(c_{0}, c\right)}, F_{\bullet-c_{0}}^{\text {ord }}\right) \longrightarrow\left(M_{\widetilde{A}}^{0}, F_{\bullet}^{\text {ord }}\right), \quad P \longmapsto P \cdot \partial^{\left(c_{0}, c\right)}
$$

(where $\partial^{\left(c_{0}, c\right)}$ is as above) is strictly filtered.

Proof. Since both filtered modules $\left(M_{\widetilde{A}}^{-\left(c_{0}, c\right)}, F_{\bullet-c_{0}}^{\text {ord }}\right)$ and $\left(M_{\widetilde{A}}^{0}, F_{\bullet}^{\text {ord }}\right)$ underly mixed Hodge modules on $V$ by Theorem 5.35 and the morphism is induced from a morphism in $\operatorname{MHM}(V, \mathbb{C})$, we obtain the strictness statement we are looking for.

Remark 5.38. If $\mathbb{C}[\mathbb{N} \widetilde{A}]$ is not Gorenstein but normal (and therefore Cohen-Macaulay), then the proof of Theorem 5.36 shows that

$$
\mathbb{D} M_{\widetilde{A}}^{\widetilde{\beta}} \simeq \mathcal{H}^{0}\left(E+\beta, D_{V} \otimes \operatorname{Ext}^{n-d}\left(S_{\widetilde{A}}, \omega_{\mathbb{C}\left[\partial_{\lambda}\right]}\right)\right) \simeq \mathcal{H}^{0}\left(E+\beta, D_{V} \otimes \omega_{S_{\widetilde{A}}}\right) .
$$

Recall that the canonical module $\omega_{S_{\widetilde{A}}}$ of $S_{\widetilde{A}}$ is isomorphic to $\mathbb{C}[\operatorname{int}(\mathbb{N} \widetilde{A})]$ in the category of $\mathbb{Z}^{d+1}$ graded $\mathbb{C}\left[\underline{\partial}_{\lambda}\right]$-modules. The module $\omega_{S_{\widetilde{A}}}$ carries a $\mathbb{Z}$-grading given by the first component of the $\mathbb{Z}^{d+1}$-grading. Hence, $D_{V} \otimes_{\mathbb{C}\left[\underline{\partial}_{\lambda}\right]} \omega_{S_{\widetilde{A}}}$ carries an order filtration which induces a filtration $F_{\bullet}^{\text {ord }}$ on $\mathcal{H}^{0}\left(E+\beta, D_{V} \otimes \omega_{S_{\widetilde{A}}}\right)$. We therefore get

$$
F_{p}^{H} \mathbb{D} \mathcal{M}^{\widetilde{\beta}} \simeq F_{p-n-c_{0}}^{\mathrm{ord}} \mathcal{H}^{0}\left(E+\beta, D_{V} \otimes \omega_{S_{\widetilde{A}}}\right),
$$

where $c_{0}:=\min \left\{\operatorname{deg}_{\mathbb{Z}}(P) \mid P \in \mathbb{C}[\operatorname{int}(\mathbb{N} A)]\right\}$. Let $\widetilde{c} \in \operatorname{deg}(\mathbb{C}[\operatorname{int}(\mathbb{N} \widetilde{A})])$ with $\widetilde{c}=\left(c_{0}, c\right)$. As done in the proof of [Wal07, Proposition 4.4], it can be shown that the inclusion $S_{\widetilde{A}}[-\widetilde{c}] \hookrightarrow$ $\mathbb{C}[\operatorname{int}(\mathbb{N} \widetilde{A})]$ induces an isomorphism $M^{-\widetilde{\beta}-\widetilde{c}} \stackrel{\sim}{\rightarrow} \mathcal{H}^{0}\left(E+\beta, D_{V} \otimes \omega_{S_{\widetilde{A}}}\right)$; however, we do not $\operatorname{expect}\left(M^{-\widetilde{\beta}-\widetilde{c}}, F_{\bullet}^{\text {ord }}\right) \rightarrow\left(\mathcal{H}^{0}\left(E+\beta, D_{V} \otimes \omega_{S_{\widetilde{A}}}\right), F_{\bullet}^{\text {ord }}\right)$ to be a filtered isomorphism.

\subsection{Hodge structures on affine hypersurfaces of tori}

In this subsection, we explain how our main result implies in a rather direct way a classical theorem of Batyrev concerning the description of the Hodge filtration of the relative cohomology of smooth affine hypersurfaces in algebraic tori. 


\section{Hypergeometric Hodge modules}

We first want to recall the sheaf-theoretic definition of relative cohomology. Let $X$ be a topological space and $K$ be a closed subset. Denote by $j: X \backslash K \rightarrow X$ the open embedding of the complement. The relative cohomology of the pair $(X, K)$ is defined as the hypercohomology

$$
H^{i}(X, K ; \mathbb{C}):=\mathbb{H}^{i}\left(X, j_{!} j^{-1} \mathbb{Q}_{X}\right) .
$$

If $X$ and $K$ are quasi-projective varieties, the relative cohomology of the pair $(X, K)$ carries a mixed Hodge structure, which is given by $\mathbb{H}^{i}\left(X, j_{!} j^{-1} \mathbb{Q}_{X}^{H}\right)$.

We want to compute this in the following situation: Consider, as in Section 5 , the family of Laurent polynomials $\varphi_{A}: T \times \Lambda \rightarrow V=\mathbb{C}_{\lambda_{0}} \times \Lambda$, where $\mathbb{Z} \widetilde{A}=\mathbb{Z}^{d+1}$ and $\mathbb{N} \widetilde{A}=\mathbb{Z}^{d+1} \cap \mathbb{R}_{\geqslant 0} \widetilde{A}$. Let $\Delta:=\operatorname{Conv}\left(\underline{a}_{0}, \underline{a}_{1}, \ldots, \underline{a}_{n}\right)$ be the convex hull of the exponents of $\varphi_{A}$, where $\underline{a}_{0}:=0$. Let $\tau \subset \Delta$ be a face of $\Delta, x \in V$ and

$$
F_{A, x}^{\tau}=\sum_{i: \underline{a}_{i} \in \tau} x_{i} \underline{\underline{t}}^{\underline{a}_{i}}
$$

Definition 5.39. The fiber $\varphi_{A}^{-1}(x)$ is non-degenerate if for every face $\tau$ of $\Delta$, the equations

$$
F_{A, x}^{\tau}=t_{1} \frac{\partial F_{A, x}^{\tau}}{\partial t_{1}}=\cdots=t_{d} \frac{\partial F_{A, x}^{\tau}}{\partial t_{d}}=0
$$

have no common solution in $T$.

Let $x \in V$ be such that the fiber $\varphi_{A}^{-1}(x)$ is non-degenerate. We give a model of $H^{i}\left(T, \varphi_{A}^{-1}(x) ; \mathbb{C}\right)$ as the quotient of a graded semigroup ring and compute explicitly its Hodge filtration. This recovers a result of Stienstra [Sti98, Theorem 7] using results of Batyrev [Bat93].

Lemma 5.40. Let $x \in V$, and let $i_{x}:\{x\} \rightarrow V$ be the inclusion. Suppose that $\varphi_{A}^{-1}(x)$ is non-degenerate.

(i) The fiber $\varphi_{A}^{-1}(x)$ is smooth.

(ii) The map $i_{x}$ is non-characteristic with respect to $\mathcal{M}_{\widetilde{A}}^{0}$.

Proof. The first statement follows directly from the definition for $\tau=\Delta$. The second statement follows from [Ado94, Lemma 3.3].

Consider the following diagram (cf. diagram (5.2)):

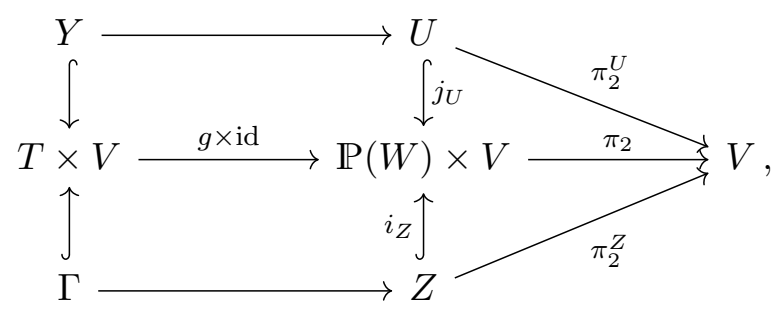

where $Y$ and $\Gamma$ are the pull-backs such that both squares on the left are cartesian. Notice that $\Gamma$ is given as a subpace of $T \times V$ by the equation $\lambda_{0}+\sum_{i=1}^{n} \lambda_{i} \underline{t}^{\underline{a}}=0$; hence, $\Gamma$ is the graph of 


\section{T. Reichelt And C. Sevenheck}

$\varphi_{A}$ and $Y$ is its complement in $T \times V$. Restricting this diagram to some $x \in V$, we therefore get

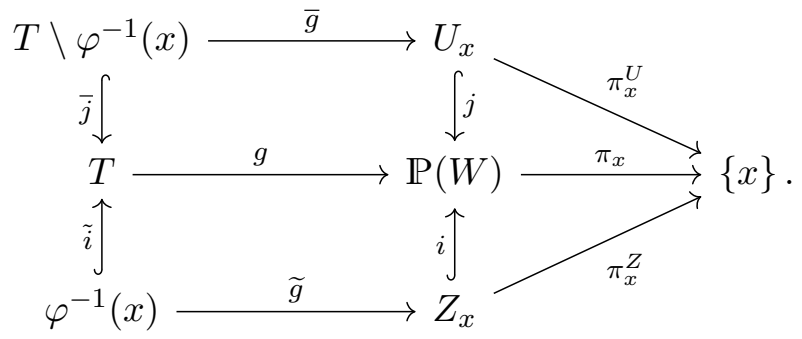

We will need the following statement.

Lemma 5.41. Let $x \in V$ be such that $\varphi_{A}^{-1}(x)$ is smooth; then we have the following isomorphism in $D^{b}(\operatorname{MHM}(\mathbb{P}(W)))$ :

$$
g_{*} \bar{j}_{!} \bar{j}^{-1} \mathbb{Q}_{T}^{H} \simeq j_{!} \bar{g}_{*} \mathbb{Q}_{T \backslash \varphi^{-1}(x)}^{H} .
$$

Proof. The statement follows using the following chain of isomorphisms:

$$
g_{*} \bar{j}_{!} \bar{j}^{-1} \mathbb{Q}_{T}^{H} \stackrel{!}{\simeq} j_{!} j^{-1} g_{*} \mathbb{Q}_{T}^{H} \simeq j_{!} \bar{g}_{*} \bar{j}^{-1} \mathbb{Q}_{T}^{H} \simeq j_{!} \bar{g}_{*} \mathbb{Q}_{T \backslash \varphi^{-1}(x)}^{H},
$$

where the second isomorphism follows from base change. It remains to show the first isomorphism. Notice that we have the two triangles

$$
j_{!} j^{-1} g_{*} \longrightarrow g_{*} \longrightarrow i_{!} i^{*} g_{*} \stackrel{+1}{\longrightarrow} \text { and } g_{*} \bar{j}_{!} \bar{j}^{-1} \longrightarrow g_{*} \longrightarrow g_{*} \bar{i}_{!} \bar{i}^{*} \stackrel{+1}{\longrightarrow} \text {. }
$$

So it is enough to show $i_{!} i^{*} g_{*} \mathbb{Q}_{T}^{H} \simeq g_{*} \tilde{i}_{i} \tilde{i}^{*} \mathbb{Q}_{T}^{H}$. But this can be seen as follows:

$$
i_{!} i^{*} g_{*} \mathbb{Q}_{T}^{H} \simeq i_{!} i^{!} g_{*} \mathbb{Q}_{T}^{H}[2] \simeq i_{!} \tilde{g}_{*} \tilde{i}^{!} \mathbb{Q}_{T}^{H}[2] \simeq i_{!} \tilde{g}_{*} \tilde{i}^{*} \mathbb{Q}_{T}^{H} \simeq g_{*} \tilde{i}_{!} \tilde{i}^{*} \mathbb{Q}_{T}^{H}
$$

where we use the smoothness of $\varphi^{-1}(x)$ in the first and third isomorphism.

In order to prove the statement that the restriction of the GKZ system is isomorphic to a relative cohomology group, we have to rewrite the GKZ system as a Radon transform. For this, consider the diagram

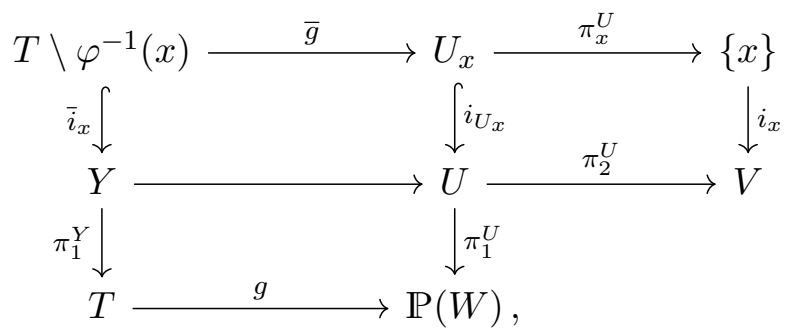

where all squares are cartesian.

Proposition 5.42. Let $x \in V$ be such that $\varphi_{A}^{-1}(x)$ is non-degenerate; then there is an isomorphism of mixed Hodge structures

$$
i_{x}^{*} \mathcal{M}_{\tilde{A}}^{0} \simeq H^{d}\left(T, \varphi_{A}^{-1}(x) ; \mathbb{C}\right),
$$

and $H^{i}\left(T, \varphi_{A}^{-1}(x) ; \mathbb{C}\right)=0$ for $i \neq d$. 


\section{Hypergeometric Hodge modules}

Proof. Consider the following isomorphisms:

$$
\begin{array}{rlrl}
i_{x}^{*} \pi_{2 !}^{U}\left(\pi_{1}^{U}\right)^{*} g_{*}^{p} \mathbb{Q}_{T}^{H} & \simeq \pi_{x !}^{U} i_{U_{x}}^{*}\left(\pi_{1}^{U}\right)^{*} g_{*}^{p} \mathbb{Q}_{T}^{H} & & \text { base change } \\
& \simeq \pi_{x !}^{U}\left(\pi_{1}^{U} \circ i_{U_{x}}\right)^{*} g_{*}{ }^{p} \mathbb{Q}_{T}^{H} & & \\
& \simeq \pi_{x !}^{U}\left(\pi_{1}^{U} \circ i_{U_{x}}\right)^{!} g_{*}{ }^{p} \mathbb{Q}_{T}^{H} & & \left(\pi_{1}^{U} \circ i_{U_{x}}\right) \text { open } \\
& \simeq \pi_{x !}^{U} \bar{g}_{*}\left(\pi_{1}^{Y} \circ \bar{i}_{x}\right)^{! p} \mathbb{Q}_{T}^{H} & & \text { base change } \\
& \simeq \pi_{x !}^{U} \bar{g}_{*}\left(\pi_{1}^{Y} \circ \bar{i}_{x}\right)^{* p} \mathbb{Q}_{T}^{H} & & \left(\pi_{1}^{Y} \circ \bar{i}_{x}\right) \text { open } \\
& \simeq \pi_{x !}^{U} \bar{g}_{*}{ }^{p} \mathbb{Q}_{T \backslash \varphi^{-1}(x)}^{H} &
\end{array}
$$

We can rewrite this further by looking at a part of diagram (5.18):

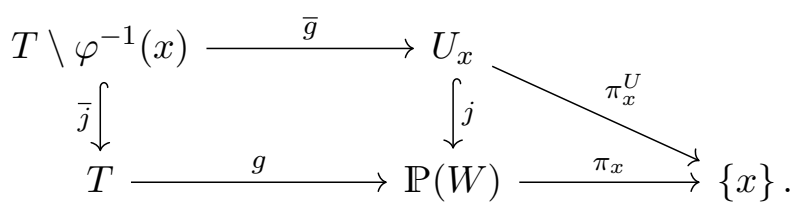

We have

$$
\pi_{x *} g_{*} \bar{j}_{!} \bar{j}^{-1}{ }^{p} \mathbb{Q}_{T}^{H} \simeq \pi_{x *} j_{!} \bar{g}_{*}^{p} \mathbb{Q}_{T \backslash \varphi^{-1}(x)}^{H} \simeq \pi_{x !}^{U} \bar{g}_{*}^{p} \mathbb{Q}_{T \backslash \varphi^{-1}(x)}^{H} \simeq i_{x}^{*} \pi_{2 !}^{U}\left(\pi_{1}^{U}\right)^{*} g_{*}{ }^{p} \mathbb{Q}_{T}^{H},
$$

where the last isomorphism follows from the calculation above. If we take cohomology and keep in mind that $i_{x}$ is non-characteristic, we get

$$
\begin{aligned}
H^{i}\left(T, \varphi_{A}^{-1}(x) ; \mathbb{C}\right) & \simeq H^{i-d}\left(\pi_{x *} g_{*} \bar{j}_{!} \bar{j}^{-1}{ }^{p} \mathbb{Q}_{T}^{H}\right) \\
& \simeq \mathcal{H}^{i-d} i_{x}^{*}\left(\pi_{2 !}^{U}\left(\pi_{1}^{U}\right)^{*} g_{*}{ }^{p} \mathbb{Q}_{T}^{H}\right) \\
& \simeq i_{x}^{*} \mathcal{H}^{i-d+n+1}\left(\pi_{2 !}^{U}\left(\pi_{1}^{U}\right)^{*} g_{*}^{p} \mathbb{Q}_{T}^{H}\right) \\
& \simeq i_{x}^{*} \mathcal{H}^{i-d+n+1}\left({ }^{*} \mathcal{R}_{c}^{\circ}\left(g_{*}{ }^{p} \mathbb{Q}_{T}^{H}\right)\right) .
\end{aligned}
$$

Since $\mathcal{H}^{k}\left({ }^{*} \mathcal{R}_{c}^{\circ}\left(g_{*}{ }^{p} \mathbb{Q}_{T}^{H}\right)\right)=0$ for $k \neq n+1$ and $\mathcal{H}^{k}\left({ }^{*} \mathcal{R}_{c}^{\circ}\left(g_{*}{ }^{p} \mathbb{Q}_{T}^{H}\right)\right)=\mathcal{M}_{\tilde{A}}^{0}$ for $k=n+1$, the claim follows.

Denote by $S_{\widetilde{A}}:=\mathbb{C}[\mathbb{N} \widetilde{A}] \subset \mathbb{C}\left[u_{0}^{ \pm}, \ldots, u_{d}^{ \pm}\right]$the semigroup ring generated by $\underline{u}^{\underline{a_{0}}}, \ldots, \underline{u}^{\underline{\tilde{a}}}{ }$, where $\widetilde{A}=\left(\underline{\tilde{a}}_{0}, \ldots, \underline{\tilde{a}}_{n}\right)$ is the matrix from (5.1).

Define the differential operators

$$
D_{k}:=\sum_{i=0}^{n}\left(\tilde{a}_{k i} x_{i} \underline{u}^{\widetilde{a}_{i}}+u_{i} \partial_{u_{i}}\right) \quad \text { for } k=0, \ldots, d \text { and fixed } x=\left(x_{0}, \ldots, x_{n}\right) \in V,
$$

which act on $\mathbb{C}\left[u_{0}^{ \pm}, \ldots, u_{d}^{ \pm}\right]$and which preserve $S_{\widetilde{A}}$. For $\underline{u}^{\underline{l}}=\underline{u}^{l_{0}} \cdot \widetilde{a}_{0} \cdot \ldots \cdot \underline{u}^{l_{n} \cdot \widetilde{a}_{n}} \in S_{\widetilde{A}}$, we define the degree $\operatorname{deg}\left(\underline{u}^{\underline{l}}\right)=\sum_{i=0}^{n} l_{i}$. Define a descending filtration $F^{\bullet}$ of $\mathbb{C}$-vector spaces on $S_{\widetilde{A}}$, where $F^{d+1} S_{\widetilde{A}}=0$ and the filtration step $F^{d-k} S_{\widetilde{A}}$ is spanned by monomials $\underline{u}^{\underline{l}}$ with $\operatorname{deg}\left(\underline{u}^{-}\right) \leqslant k$.

THEOREM 5.43. Let $x \in V$ be such that $\varphi_{A}^{-1}(x)$ is non-degenerate; then the following isomorphism of filtered vector spaces holds:

$$
\left(i_{x}^{*} \mathcal{M}_{\widetilde{A}}^{0}, F_{\bullet}^{H}\right) \simeq\left(S_{\widetilde{A}} /\left(D_{k} S_{\widetilde{A}}\right)_{k=0, \ldots, d}, F^{-\bullet}\right) .
$$

Proof. Since we have assumed that $i_{x}$ is non-characteristic with respect to $\mathcal{M}_{\widetilde{A}}^{0}$, the only non-zero cohomology group of $i_{x}^{*} \mathcal{M}_{\widetilde{A}}^{0}$ is $\mathcal{H}^{0} i_{x}^{*} \mathcal{M}_{\widetilde{A}}^{0} \simeq\left(\lambda_{i}-x_{i}\right)_{i=0, \ldots, n} \backslash \mathcal{M}_{\widetilde{A}}^{0}$. 


\section{T. Reichelt and C. Sevenheck}

We define a $\mathbb{C}$-linear map

$$
\Psi^{\prime}: S_{\widetilde{A}} \longrightarrow\left(\lambda_{i}-x_{i}\right)_{i=0, \ldots, n} \backslash \mathcal{M}_{\widetilde{A}}^{0}, \quad \underline{u}^{l_{0} \cdot \widetilde{a}_{0}} \cdot \ldots \cdot \underline{u}^{l_{n} \cdot \widetilde{a}_{n}} \longmapsto \partial_{\lambda_{0}}^{l_{0}} \cdot \ldots \cdot \partial_{\lambda_{n}}^{l_{n}} .
$$

We want to show that this map factors over $S_{\widetilde{A}} /\left(D_{k} S_{\widetilde{A}}\right)_{k=0, \ldots, d}$, so that $\Psi^{\prime}$ descends to a map

$$
\Psi: S_{\widetilde{A}} /\left(D_{k} S_{\widetilde{A}}\right)_{k=0, \ldots, d} \longrightarrow\left(\lambda_{i}-x_{i}\right)_{i=0, \ldots, n} \backslash \mathcal{M}_{\widetilde{A}}^{0} .
$$

Let $P=\underline{u}^{l_{0} \cdot \underline{\tilde{a}}_{0}} \cdot \ldots \cdot \underline{u}^{l_{n} \cdot \underline{a}_{n}} ;$ then $\Psi^{\prime}(P)=\partial_{\lambda_{0}}^{l_{0}} \cdot \ldots \cdot \partial_{\lambda_{n}}^{l_{n}}$ and

$$
\begin{aligned}
\Psi^{\prime}\left(D_{k} P\right) & =\Psi\left(\sum_{i=0}^{n} \tilde{a}_{k i} x_{i} \underline{u}_{i} P+\sum_{i=0}^{n} \tilde{a}_{k i} l_{i} P\right) \\
& =\left(\sum_{i=0}^{n} \tilde{a}_{k i} x_{i} \partial_{\lambda_{i}}+\sum_{i=0}^{n} \tilde{a}_{k i} l_{i}\right) \Psi(P)=\left(\sum_{i=0}^{n} \tilde{a}_{k i} \lambda_{i} \partial_{\lambda_{i}}+\sum_{i=0}^{n} \tilde{a}_{k i} l_{i}\right) \Psi(P) \\
& =\Psi(P) \cdot\left(\sum_{i=0}^{n} \tilde{a}_{k i} \lambda_{i} \partial_{\lambda_{i}}\right)=0 .
\end{aligned}
$$

We will now construct an inverse $\Theta$ to $\Psi$. If $P \in D_{V}$ is a normally ordered element, we denote by $\bar{P} \in S_{\widetilde{A}}$ the element which is obtained from $P$ by replacing $\lambda_{i}$ with $x_{i}$ and $\partial_{\lambda_{i}}$ with $\underline{u}^{l_{i} \underline{a}_{i}}$; that is, if $P=\lambda_{0}^{k_{0}} \cdots \lambda_{n}^{k_{n}} \partial_{\lambda_{0}}^{l_{0}} \cdots \partial_{\lambda_{n}}^{l_{n}}$, the element $\bar{P}$ is given by $x_{0}^{k_{0}} \cdots x_{n}^{k_{n}} \underline{u}^{l_{0} \cdot \underline{a}_{0}} \cdots \underline{u}^{l_{n} \cdot \underline{a}_{n}}$. This gives the map

$$
\Theta^{\prime}:\left(\lambda_{i}-x_{i}\right)_{i=0, \ldots, n} \backslash D_{V} \longrightarrow S_{\widetilde{A}} /\left(D_{k} S_{\widetilde{A}}\right)_{k=0, \ldots, d}, \quad P \longmapsto \bar{P}
$$

We want to show that $\Theta^{\prime}$ factors over $\left(\lambda_{i}-x_{i}\right)_{i=0, \ldots, n} \backslash M_{A}^{0}$, so that $\Theta^{\prime}$ descends to a map

$$
\Theta:\left(\lambda_{i}-x_{i}\right)_{i=0, \ldots, n} \backslash M_{A}^{0} \longrightarrow S_{\widetilde{A}} /\left(D_{k} S_{\widetilde{A}}\right)_{k=0, \ldots, d}, \quad P \longmapsto \bar{P} .
$$

We have to show that $\Theta^{\prime}\left(P \cdot E_{k}\right)=0$ and $\Theta^{\prime}\left(P \cdot \square_{\underline{l}}\right)=0$ for $k=0, \ldots, d$ and $l \in \mathbb{L}$. We can assume that $P$ is a monomial $\lambda_{0}^{j_{0}} \cdots \lambda_{n}^{j_{n}} \partial_{\lambda_{0}}^{l_{0}} \cdots \partial_{\lambda_{n}}^{l_{n}}$ :

$$
\begin{aligned}
\Theta^{\prime}\left(\lambda_{0}^{j_{0}} \cdots \lambda_{n}^{j_{n}} \partial_{\lambda_{0}}^{l_{0}} \cdots \partial_{\lambda_{n}}^{l_{n}} E_{k}\right) & =\Theta^{\prime}\left(\lambda_{0}^{j_{0}} \cdots \lambda_{n}^{j_{n}} \partial_{\lambda_{0}}^{l_{0}} \cdots \partial_{\lambda_{n}}^{l_{n}}\left(\sum_{i=0}^{n} \tilde{a}_{k i} \lambda_{i} \partial_{\lambda_{i}}\right)\right) \\
& =\Theta^{\prime}\left(\lambda_{0}^{j_{0}} \cdots \lambda_{n}^{j_{n}}\left(\sum_{i=0}^{n}\left(\tilde{a}_{k i} \lambda_{i} \partial_{\lambda_{i}}+\tilde{a}_{k i} l_{i}\right)\right) \partial_{\lambda_{0}}^{l_{0}} \cdots \partial_{\lambda_{n}}^{l_{n}}\right) \\
& =x_{0}^{j_{0}} \cdots x_{n}^{j_{n}}\left(\sum_{i=0}^{n}\left(\tilde{a}_{k i} x_{i} \underline{u}^{\underline{a}_{i}}+\tilde{a}_{k i} l_{i}\right)\right) \underline{u}^{l_{0} \cdot \underline{a}_{0}} \cdots \underline{u}^{l_{n} \cdot \underline{a}_{0}} \\
& =\left(\sum_{i=0}^{n}\left(\tilde{a}_{k i} x_{i} \underline{u}^{\underline{a_{i}}}+\tilde{a}_{k i} l_{i}\right)\right) \cdot x_{0}^{j_{0}} \cdots x_{n}^{j_{n}} \cdot \underline{u}^{l_{0} \cdot \underline{a}_{0}} \cdots \underline{u}^{l_{n} \cdot \underline{a}_{0}} \\
& =\left(\sum_{i=0}^{n}\left(\tilde{a}_{k i} x_{i} \underline{u}^{\underline{a}_{i}}+u_{i} \partial_{u_{i}}\right)\right) \cdot x_{0}^{j_{0}} \cdots x_{n}^{j_{n}} \cdot \underline{u}^{l_{0} \cdot \underline{a}_{0}} \cdots \underline{u}^{l_{n} \cdot \underline{a}_{0}} \\
& =0
\end{aligned}
$$

and

$$
\Theta^{\prime}\left(\lambda_{0}^{j_{0}} \cdots \lambda_{n}^{j_{n}} \partial_{\lambda_{0}}^{l_{0}} \cdots \partial_{\lambda_{n}}^{l_{n}} \square_{\underline{l}}\right)=\Theta^{\prime}\left(\lambda_{0}^{j_{0}} \cdots \lambda_{n}^{j_{n}} \partial_{\lambda_{0}}^{l_{0}} \cdots \partial_{\lambda_{n}}^{l_{n}}\left(\prod_{l_{i}>0} \partial_{\lambda_{i}}^{l_{i}}-\prod_{l_{i}<0} \partial_{\lambda_{i}}^{-l_{i}}\right)\right)
$$




\section{Hypergeometric Hodge modules}

$$
\begin{aligned}
& =x_{0}^{j_{0}} \cdots x_{n}^{j_{n}} \underline{u}^{l_{0} \cdot \underline{a}_{0}} \cdots \underline{u}^{l_{n} \cdot \underline{a}_{n}}\left(\prod_{l_{i}>0} \underline{u}^{l_{i} \cdot \underline{a}_{i}}-\prod_{l_{i}<0} \underline{u}^{-l_{i} \cdot \underline{a}_{i}}\right) \\
& =x_{0}^{j_{0}} \cdots x_{n}^{j_{n}} \underline{u}^{l_{0} \cdot \underline{a}_{0}} \cdots \underline{u}^{l_{n} \cdot \underline{a}_{n}}\left(\underline{u}^{\sum_{l_{i}>0} l_{i} \cdot \underline{a}_{i}}-\underline{u}^{-\sum_{l_{i}<0} l_{i} \cdot \underline{a}_{i}}\right) \\
& =0,
\end{aligned}
$$

where the last equality follows from $\sum_{i=0}^{n} l_{i} \underline{a}_{i}=0$. This shows that $\Psi$ is an isomorphism. The statement about the Hodge filtration follows from Theorem 5.35, the fact that $x$ is a smooth point of $\mathcal{M}_{\widetilde{A}}^{0}$ and the definition of the inverse image of filtered $\mathcal{D}$-modules (cf. [Sai88, Chapter 3.5]; notice that no shift in the Hodge filtration occurs since we are dealing with left $\mathcal{D}$-modules instead of right $\mathcal{D}$-modules as in [Sai88]).

\section{Landau-Ginzburg models and non-commutative Hodge structures}

In this final section, we will give a first application of our main result. It is concerned with Hodgetheoretic properties of differential systems occurring in toric mirror symmetry. More precisely, we will prove [RS17, Conjecture 6.15], showing that the so-called reduced quantum $\mathcal{D}$-module of a nef (also called weak Fano) complete intersection inside a smooth projective toric variety underlies a (variation of) non-commutative Hodge structure(s). We will recall the necessary notation and results of [RS17] and then deduce this conjecture from our main theorem, Theorem 5.35. The basic strategy to obtain the proof of the conjecture is to identify the reduced quantum $\mathcal{D}$ module with an object which is the Fourier-Laplace transform of a filtered $\mathcal{D}$-module underlying a pure polarized Hodge module. The latter is nothing but the image of the duality morphism from Corollary 5.37. Notice that this corollary depends in an essential way on our main Theorem 5.35 since the strictness of the duality morphism with respect to the order filtrations holds only because the latter are (up to a shift) the Hodge filtrations of mixed Hodge modules. The identification with the reduced quantum $\mathcal{D}$-module relies on the explicit description of the latter from [MM17] (already used extensively in [RS17]).

Let $X_{\Sigma}$ be a smooth, projective and toric variety with $\operatorname{dim}_{\mathbb{C}}\left(X_{\Sigma}\right)=k$. Put $m:=k+b_{2}\left(X_{\Sigma}\right)$. Let $\mathcal{L}_{1}, \ldots, \mathcal{L}_{l}$ be globally generated line bundles on $X_{\Sigma}$ (in particular, they are nef according to [Ful93, Section 3.4]), and assume that $-K_{X_{\Sigma}}-\sum_{i=1}^{l} c_{1}\left(\mathcal{L}_{i}\right)$ is nef. Put $\mathcal{E}:=\oplus_{i=1}^{l} \mathcal{L}_{i}$, and let $\mathcal{E}^{\vee}$ be the dual vector bundle. Its total space $\mathbb{V}\left(\mathcal{E}^{\vee}\right):=\operatorname{Spec}_{\mathcal{O}_{X_{\Sigma}}}\left(\operatorname{Sym}_{\mathcal{O}_{X_{\Sigma}}}(\mathcal{E})\right)$ is a quasi-projective toric variety with defining fan $\Sigma^{\prime}$. The matrix $A \in M((k+l) \times(m+l), \mathbb{Z})$ whose columns are the primitive integral generators of the rays of $\Sigma^{\prime}$ then satisfies the conditions in Theorem 5.36. More precisely, we have $\mathbb{Z} \widetilde{A}=\mathbb{Z}^{d+1}$, and it follows from $[\mathrm{RS} 17$, Proposition 5.1] that the semigroup $\mathbb{N} \widetilde{A}$ is normal and that we have int $(\mathbb{N} \widetilde{A})=\widetilde{c}+\mathbb{N} \widetilde{A}$, where $\widetilde{c}=\sum_{i=m+1}^{m+l} e_{i}=(l+1, \underline{0}, \underline{1})$, with $e_{i}$ the $i$ th standard vector in $\mathbb{Z}^{1+m+l}$.

The strictly filtered duality morphism $\phi$ from Corollary 5.37 is more concretely given as

$$
\phi:\left(\mathcal{M}_{\widetilde{A}}^{-(l+1, \underline{0}, \underline{1})}, F_{\bullet-l-1}^{\mathrm{ord}}\right) \longrightarrow\left(\mathcal{M}_{\widetilde{A}}^{0}, F_{\bullet}^{\text {ord }}\right), \quad P \longmapsto P \cdot \partial_{\lambda_{0}} \cdot \partial_{\lambda_{m+1}} \cdot \ldots \cdot \partial_{\lambda_{m+l}} \cdot
$$

Proposition 6.1. The image of $\phi$ underlies a pure Hodge module of weight $m+k+2 l$, where the Hodge filtration is given by

$$
F_{\bullet}^{H} \operatorname{im}(\phi)=\operatorname{im}(\phi) \cap F_{\bullet+k+l}^{\mathrm{ord}} \mathcal{M}_{\widetilde{A}}^{0} .
$$

Proof. This is a consequence of [RS17, Theorem 2.16] and Proposition 5.2.

A main point in the paper [RS17] is to consider the partial localized Fourier transformations 


\section{T. Reichelt And C. Sevenheck}

of the GKZ systems $\mathcal{M}_{\widetilde{A}}^{\beta}$. We recall the main construction and refer to [RS17, Section 3.1] for details (in particular concerning the definition and properties of the Fourier-Laplace functor FL and its "localized" version $\left.\mathrm{FL}^{\text {loc }}\right)$. As in Subsection 5.1 , let $\Lambda$ be the affine space $\mathbb{C}^{m+l}$ with coordinates $\lambda_{1}, \ldots, \lambda_{m+l}$ (so that $V=\mathbb{C}_{\lambda_{0}} \times \Lambda$ ), and put $\widehat{V}:=\mathbb{C}_{z} \times \Lambda$. Let $\widehat{\mathcal{M}}_{A}^{\left(\beta_{0}, \beta\right)}$ be the $\mathcal{D}_{\widehat{V}}$-module $\mathcal{D}_{\widehat{V}}\left[z^{-1}\right] / \mathcal{I}$, where $\mathcal{I}$ is the left ideal generated by the operators $\widehat{\square}_{\underline{l}}$ (for all $l \in \mathbb{L}_{A}$ ), $\widehat{E}_{j}-\beta_{j} z$ (for $j=1, \ldots, k+l$ ) and $\widehat{E}-\beta_{0} z$, which are defined by

$$
\begin{aligned}
\widehat{\square}_{\underline{l}} & :=\prod_{i: l_{i}<0}\left(z \cdot \partial_{\lambda_{i}}\right)^{-l_{i}}-\prod_{i: l_{i}>0}\left(z \cdot \partial_{\lambda_{i}}\right)^{l_{i}}, \\
\widehat{E}_{j} & :=\sum_{i=1}^{m+l} a_{j i} z \lambda_{i} \partial_{\lambda_{i}}, \quad \widehat{E}:=z^{2} \partial_{z}+\sum_{i=1}^{m+l} z \lambda_{i} \partial_{\lambda_{i}} .
\end{aligned}
$$

We denote the corresponding $\mathcal{D}_{\widehat{V}}$-module by $\widehat{\mathcal{M}}_{A}^{\left(\beta_{0}, \beta\right)}$. Then we have [RS17, Lemma 3.2]

$$
\mathrm{FL}_{\Lambda}^{\text {loc }}\left(\mathcal{M}_{\widetilde{A}}^{\left(\beta_{0}, \beta\right)}\right)=\widehat{\mathcal{M}}_{A}^{\left(\beta_{0}+1, \beta\right)} .
$$

Consider the filtration on $\mathcal{D}_{\widehat{V}}$ for which $\operatorname{deg}(z)=-1, \operatorname{deg}\left(\partial_{z}\right)=2, \operatorname{deg}\left(\lambda_{i}\right)=0$ and $\operatorname{deg}\left(\partial_{\lambda_{i}}\right)=1$. Write $\operatorname{MF}^{z}\left(\mathcal{D}_{\widehat{V}}\right)$ for the category of well-filtered $\mathcal{D}_{\widehat{V}}$-modules (that is, $\mathcal{D}_{\widehat{V}}$-modules equipped with a filtration compatible with the filtration on $\mathcal{D}_{\widehat{V}}$ just described and such that the corresponding Rees module is coherent over the corresponding Rees ring). Denote by $G_{\bullet}$ the induced filtrations on the module $\widehat{\mathcal{M}}_{A}^{\left(\beta_{0}, \beta\right)}$, which are $\mathcal{R}_{\mathbb{C}_{z} \times \Lambda}$-modules. We have

$$
G_{0} \widehat{\mathcal{M}}_{A}^{\left(\beta_{0}, \beta\right)}=\mathcal{R}_{\mathbb{C}_{z} \times \Lambda} / \mathcal{R}_{\mathbb{C}_{z} \times \Lambda}\left(\widehat{\square}_{\underline{l}}\right)_{l \in \mathbb{L}_{A}}+\mathcal{R}_{\mathbb{C}_{z} \times \Lambda} \widehat{E}+\mathcal{R}_{\mathbb{C}_{z} \times \Lambda}\left(\widehat{E}_{j}\right)_{k=1, \ldots, k+l}
$$

and $G_{k} \widehat{\mathcal{M}}_{A}^{\left(\beta_{0}, \beta\right)}=z^{k} \cdot G_{0} \widehat{\mathcal{M}}_{A}^{\left(\beta_{0}, \beta\right)}$ In general, the modules $\widehat{\mathcal{M}}_{A}^{\left(\beta_{0}, \beta\right)}$ and their filtration steps may be quite complicated. However, in [RS17], we have considered their restriction to a specific Zariskiopen subset $\Lambda^{\circ} \subset\left(\Lambda \backslash \bigcup_{i=1}^{m+l}\left\{w_{i}=0\right\}\right) \subset \Lambda$ (called $W^{\circ}$ in [RS17, Remark 3.8]), which contains the critical locus of the family of Laurent polynomials associated with the matrix $A$ (but excludes certain singularities at infinity of this family). Denote by ${ }^{\circ} \widehat{\mathcal{M}}_{A}^{\left(\beta_{0}, \beta\right)}$ the restriction $\left(\widehat{\mathcal{M}}_{A}^{\left(\beta_{0}, \beta\right)}\right)_{\mid \mathbb{C}_{z} \times \Lambda^{\circ}}$

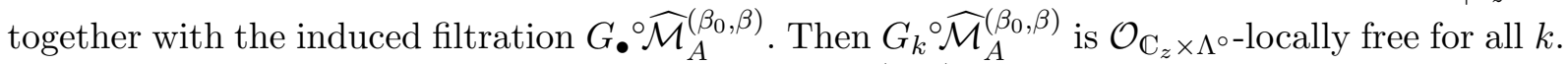
Moreover, the multiplication by $z$ is invertible on $\widehat{\mathcal{M}}_{A}^{\left(\beta_{0}, \beta\right)}$, filtered with respect to $G_{\bullet}$ (shifting the filtration by 1 ), and so is its inverse. Hence, we have a strict morphism

$$
\cdot z:\left({ }^{\circ} \widehat{\mathcal{M}}_{A}^{\left(\beta_{0}, \beta\right)}, G_{\bullet}\right) \longrightarrow\left({ }^{\circ} \widehat{\mathcal{M}}_{A}^{\left(\beta_{0}-1, \beta\right)}, G_{\bullet+1}\right) .
$$

We also need a slightly modified version of the Fourier-Laplace transformed GKZ systems. More precisely, define the modules ${ }^{\circ} \widehat{\mathcal{N}}_{A}^{\beta}$ as the cyclic quotients of $\mathcal{D}_{\mathbb{C}_{z} \times \Lambda^{\circ}}\left[z^{-1}\right]$ by the left ideal generated by $\widetilde{\square}_{\underline{l}}$ for $\underline{l} \in \mathbb{L}_{A}$ and $\widehat{E}_{j}-z \beta_{j}$ for $j=0, \ldots, k+c$, where

$$
\begin{aligned}
\widetilde{\square}_{\underline{l}}:= & \prod_{i \in\{1, \ldots, m\}: l_{i}>0} \lambda_{i}^{l_{i}}\left(z \cdot \partial_{i}\right)^{l_{i}} \prod_{i \in\{m+1, \ldots, m+l\}: l_{i}>0} \prod_{\nu=1}^{l_{i}}\left(\lambda_{i}\left(z \cdot \partial_{i}\right)-z \cdot \nu\right) \\
& -\prod_{i=1}^{m+l} \lambda_{i}^{l_{i}} \cdot \prod_{i \in\{1, \ldots, m\}: l_{i}<0} \lambda_{i}^{-l_{i}}\left(z \cdot \partial_{i}\right)^{-l_{i}} \prod_{i \in\{m+1, \ldots, m+l\}: l_{i}<0} \prod_{\nu=1}^{-l_{i}}\left(\lambda_{i}\left(z \cdot \partial_{i}\right)-z \cdot \nu\right) .
\end{aligned}
$$

Consider the invertible morphism

$$
\Psi:{ }^{\circ} \widehat{\mathcal{N}}_{A}^{(0, \underline{0}, \underline{0})} \longrightarrow{ }^{\circ} \widehat{\mathcal{M}}_{A}^{-(2 l, \underline{0}, \underline{1})}
$$




\section{Hypergeometric Hodge modules}

given by right multiplication with $z^{l} \cdot \prod_{i=m+1}^{m+l} \lambda_{i}\left(\right.$ recall that $\lambda_{i} \neq 0$ on $\left.\Lambda^{\circ}\right)$. We define $\widetilde{\phi}$ to be the composition $\widetilde{\phi}:=\widehat{\phi} \circ \Psi$, where $\widehat{\phi}$ is the morphism

$$
\widehat{\phi}:{ }^{\circ} \widehat{\mathcal{M}}_{A}^{-(2 l, \underline{0}, \underline{1})} \longrightarrow{ }^{\circ} \widehat{\mathcal{M}}_{A}^{(-l, \underline{0}, \underline{0})}
$$

given by right multiplication with $\partial_{\lambda_{m+1}} \cdot \ldots \cdot \partial_{\lambda_{m+l}}$. In concrete terms, we have

$$
\begin{aligned}
\widetilde{\phi}:{ }^{\circ} \widehat{\mathcal{N}}_{A}^{(0, \underline{0}, \underline{0})} & \longrightarrow \widehat{\mathcal{M}}_{A}^{(-l, \underline{0}, \underline{0})}, \\
x & \longmapsto \widehat{\phi}\left(x \cdot z^{l} \cdot \lambda_{m+1} \cdot \ldots \cdot \lambda_{m+l}\right)=x \cdot\left(z \lambda_{m+1} \partial_{m+1}\right) \cdot \ldots \cdot\left(z \lambda_{m+l} \partial_{m+l}\right) .
\end{aligned}
$$

We have an induced filtration $G \bullet \widehat{\mathcal{N}}_{A}^{(0, \underline{0}, \underline{0})}$ which satisfies

$$
G_{0}^{\circ} \widehat{\mathcal{N}}_{A}^{(0, \underline{0}, \underline{0})}=\mathcal{R}_{\mathbb{C}_{z} \times \Lambda^{\circ}} / \mathcal{R}_{\mathbb{C}_{z} \times \Lambda^{\circ}}\left(\widetilde{\square}_{\underline{l}}\right)_{\underline{l} \in \mathbb{L}_{A}}+\mathcal{R}_{\mathbb{C}_{z} \times \Lambda^{\circ}}\left(\widehat{E}_{j}-z \beta_{j}\right)_{j=0, \ldots, m+l}
$$

and $G_{k} \circ \widehat{\mathcal{N}}_{A}^{(0, \underline{0}, \underline{0})}=z^{k} \cdot G_{0} \circ \widehat{\mathcal{N}}_{A}^{(0, \underline{0}, \underline{0})}$

In order to obtain the lattices $G_{\bullet}$, we need to extend the functor $\mathrm{FL}_{\Lambda}^{\text {loc }}$ to the category of filtered $\mathcal{D}$-modules.

Definition 6.2. Let $\left(\mathcal{M}, F_{\bullet}\right) \in \operatorname{MF}\left(\mathcal{D}_{V}\right)=\operatorname{MF}\left(\mathcal{D}_{\mathbb{C}_{\lambda_{0}} \times \Lambda}\right)$. Define $\mathcal{M}\left[\partial_{\lambda_{0}}^{-1}\right]:=\mathcal{D}_{V}\left[\partial_{\lambda_{0}}^{-1}\right] \otimes_{\mathcal{D}_{V}} \mathcal{M}$, and consider the natural localization morphism $\widehat{\operatorname{loc}}: \mathcal{M} \rightarrow \mathcal{M}\left[\partial_{\lambda_{0}}^{-1}\right]$. We define the saturation of $F \bullet$ to be

$$
F_{k} \mathcal{M}\left[\partial_{\lambda_{0}}^{-1}\right]:=\sum_{j \geqslant 0} \partial_{\lambda_{0}}^{-j} \widehat{\operatorname{loc}}\left(F_{k+j} \mathcal{M}\right)
$$

and we denote by $G_{\bullet} \widehat{\mathcal{M}}$ the filtration induced from $F_{k} \mathcal{M}\left[\partial_{\lambda_{0}}^{-1}\right]$ on $\widehat{\mathcal{M}}:=\operatorname{FL}_{\Lambda}^{\text {loc }}(M) \in M_{h}\left(\mathcal{D}_{\widehat{V}}\right)=$ $M_{h}\left(\mathcal{D}_{\mathbb{C}_{z} \times \Lambda}\right)$. Notice that for $\left(\mathcal{M}, F_{\bullet}\right)=\left(\widehat{\mathcal{M}}_{A}^{\left(\beta_{0}, \beta\right)}, F_{\bullet}^{\text {ord }}\right)$, the two definitions of $G_{\bullet}$ coincide: as we have

$$
F_{k}^{\mathrm{ord}} \mathcal{M}_{A}^{\left(\beta_{0}-1, \beta\right)}\left[\partial_{\lambda_{0}}^{-1}\right]=\operatorname{im}\left(\partial_{\lambda_{0}}^{k} \mathbb{C}\left[\lambda_{0}, \lambda_{1}, \ldots, \lambda_{m+l}\right]\left\langle\partial_{\lambda_{0}}^{-1}, \partial_{\lambda_{0}}^{-1} \partial_{\lambda_{1}}, \ldots, \partial_{\lambda_{0}}^{-1} \partial_{\lambda_{m+l}}\right\rangle\right)
$$

in $\mathcal{M}_{\widetilde{A}}^{\left(\beta_{0}-1, \beta\right)}\left[\partial_{\lambda_{0}}^{-1}\right]$, the filtration induced by $F_{k}^{\text {ord }} \mathcal{M}_{\widetilde{A}}^{\left(\beta_{0}-1, \beta\right)}\left[\partial_{\lambda_{0}}^{-1}\right]$ on $\widehat{\mathcal{M}}_{A}^{\left(\beta_{0}, \beta\right)}$ is precisely $G_{k} \widehat{\mathcal{M}}_{A}^{\left(\beta_{0}, \beta\right)}$.

We denote by $\left(\mathrm{FL}_{\Lambda}^{\text {loc }}\right.$, Sat $)$ the induced functor from the category $\operatorname{MF}\left(\mathcal{D}_{V}\right)$ to the category $\operatorname{MF}^{z}\left(\mathcal{D}_{\widehat{\Lambda}}\right)$ which sends $\left(\mathcal{M}, F_{\bullet}\right)$ to $\left(\widehat{\mathcal{M}}, G_{\bullet}\right)$.

From the above duality considerations, we deduce the following result.

Proposition 6.3. The morphism

$$
\widetilde{\phi}:{ }^{\circ} \widehat{\mathcal{N}}_{A}^{(0, \underline{0}, \underline{0})} \longrightarrow{ }^{\circ} \widehat{\mathcal{M}}_{A}^{(-l, \underline{0}, \underline{0})}
$$

is strict with respect to the filtration $G_{\bullet}$; in particular, we have

$$
\widetilde{\phi}\left(G_{0} \widehat{\mathcal{N}}_{A}^{(0, \underline{\underline{0}} \underline{0})}\right)=G_{0}{ }^{\circ} \widehat{\mathcal{M}}_{A}^{(-l, \underline{0}, \underline{0})} \cap \operatorname{im}(\widetilde{\phi}) .
$$

Moreover, the object $\left(\operatorname{im}(\widetilde{\phi}), G_{\bullet}\right)$ is obtained via the functor $\left(\mathrm{FL}_{\Lambda}^{\text {loc }}, \operatorname{Sat}\right)$ from $\left(\operatorname{im}(\phi), F_{\bullet}^{H}=\right.$ $F_{\bullet+k+l}^{\text {ord }}$ ), which underlies a pure Hodge module of weight $m+k+2 l$ by Proposition 6.1.

Proof. The morphism $\Psi$ is invertible and filtered (shifting the filtration by $-l$ ), and its inverse is also filtered. Hence, it is strict. Therefore, the strictness of $\widetilde{\phi}$ follows from the strictness of $z \widehat{\phi}$. We will deduce the latter from the strictness property of the morphism $\phi$ in Corollary 5.37. 


\section{T. Reichelt And C. Sevenheck}

Notice that the morphism $\widehat{\phi}$ is obtained from $\phi$ by linear extension in $\partial_{\lambda_{0}}^{-1}$. Recall that the morphism

$$
\phi:\left(\mathcal{M}_{\widetilde{A}}^{-(l+1, \underline{0}, \underline{1})}, F_{\bullet}^{\text {ord }}\right) \longrightarrow\left(\mathcal{M}_{\widetilde{A}}^{0}, F_{\bullet+l+1}^{\text {ord }}\right)
$$

was strict; hence, equation (6.1) yields the strictness of

$$
\widehat{\phi}:\left(\widehat{\mathcal{M}}_{A}^{-(2 l, \underline{0}, \underline{1})}, G_{\bullet}\right) \longrightarrow\left(\widehat{\mathcal{M}}_{A}^{(-l, \underline{0}, \underline{0})}, G_{\bullet+l}\right) .
$$

Finally, as already noticed above, this yields the strictness of

$$
\widetilde{\phi}=\widehat{\phi} \circ \Psi:\left(\widehat{\mathcal{N}}_{A}^{(0, \underline{0}, \underline{0})}, G_{\bullet}\right) \longrightarrow\left(\widehat{\mathcal{M}}_{A}^{(-l, \underline{0}, \underline{0})}, G_{\bullet}\right) .
$$

The following corollary is now a direct consequence of [Sab08, Corollary 3.15]. See [Sab11] for a detailed discussion of the notion of a variation of pure polarized non-commutative Hodge structures.

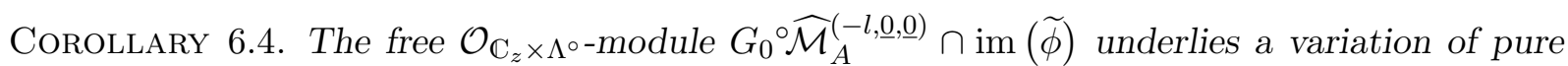
polarized non-commutative Hodge structures on $\Lambda^{\circ}$.

The main result in [RS17] concerns a mirror statement for several quantum $\mathcal{D}$-modules which are associated with the toric variety $X_{\Sigma}$ and the split vector bundle $\mathcal{E}$. In particular, one can consider the reduced quantum $\mathcal{D}$-module $\overline{\mathrm{QDM}}\left(X_{\Sigma}, \mathcal{E}\right)$ which is a vector bundle on $\mathbb{C}_{z} \times H^{0}\left(X_{\Sigma}, \mathbb{C}\right) \times B_{\varepsilon}^{*}$, where $B_{\varepsilon}^{*}:=\left\{q \in\left(\mathbb{C}^{*}\right)^{b_{2}\left(X_{\Sigma}\right)}|0<| q \mid<\varepsilon\right\}$, together with a flat connection

$$
\begin{aligned}
\nabla & : \overline{\operatorname{QDM}}\left(X_{\Sigma}, \mathcal{E}\right) \\
& \longrightarrow \overline{\mathrm{QDM}}\left(X_{\Sigma}, \mathcal{E}\right) \otimes_{\mathcal{O}_{\mathbb{C}_{z} \times H^{0}\left(X_{\Sigma}, \mathbb{C}\right) \times B_{\varepsilon}^{*}}} z^{-1} \Omega_{\mathbb{C}_{z} \times H^{0}\left(X_{\Sigma}, \mathbb{C}\right) \times B_{\varepsilon}^{*}}^{1}\left(\log \left(\{0\} \times H^{0}\left(X_{\Sigma}, \mathbb{C}\right) \times B_{\varepsilon}^{*}\right)\right) .
\end{aligned}
$$

We refer to [MM17] for a detailed discussion of the definition of $\overline{\mathrm{QDM}}\left(X_{\Sigma}, \mathcal{E}\right)$; a short version can be found in [RS17, Section 4.1]. Notice that in loc. cit., $\overline{\operatorname{QDM}}\left(X_{\Sigma}, \mathcal{E}\right)$ is defined on some larger set, but in mirror-type statements, only its restriction to $H^{0}\left(X_{\Sigma}, \mathbb{C}\right) \times \mathbb{C}_{z} \times B_{\varepsilon}^{*}$ is considered. From here on, we will need to consider a Zariski-open subset of $\mathcal{K M}^{\circ} \subset\left(\mathbb{C}^{*}\right)^{b_{2}\left(X_{\Sigma}\right)}$ which contains $B_{\varepsilon}^{*}$. We recall the main result from [MM17], which gives a GKZ-type description of $\overline{\mathrm{QDM}}\left(X_{\Sigma}, \mathcal{E}\right)$. We present it in a slightly different form, taking into account [RS17, Proposition 6.9]. Let $\mathcal{R}_{\mathbb{C}_{z} \times \mathcal{K} \mathcal{M}^{0}}$ be the sheaf of Rees rings on $\mathbb{C}_{z} \times \mathcal{K M}^{\circ}$ and $R_{\mathbb{C}_{z} \times \mathcal{K} \mathcal{M}^{0}}$ its module of global sections. If we write $q_{1}, \ldots, q_{r}$ for the coordinates on $\left(\mathbb{C}^{*}\right)^{r}$ (with $r:=b_{2}\left(X_{\Sigma}\right)$ ), then $R_{\mathbb{C}_{z} \times \mathcal{K} \mathcal{M}^{0}}$ is generated by $z q_{i} \partial_{q_{i}}$ and $z^{2} \partial_{z}$ over $\mathcal{O}_{\mathbb{C}_{z} \times \mathcal{K} \mathcal{M}^{\circ}}$.

The following result uses the notion of "quantized operator" defined in [MM17, Notation 4.2] and [RS17, Theorem 6.7].

Theorem 6.5. For any $\mathcal{L} \in \operatorname{Pic}\left(X_{\Sigma}\right)$, write $\widehat{\mathcal{L}} \in R_{\mathbb{C}_{z} \times \mathcal{K} \mathcal{M}^{\circ}}$ for the associated quantized operator. Define the left ideal $J$ of $R_{\mathbb{C}_{z} \times \mathcal{K} \mathcal{M}}$ by

$$
J:=R_{\mathbb{C}_{z} \times \mathcal{K} \mathcal{M}^{\circ}}\left(Q_{\underline{l}}\right)_{\underline{l} \in \mathbb{L}_{A^{\prime}}}+R_{\mathbb{C}_{z} \times \mathcal{K} \mathcal{M}^{\circ}} \cdot \widehat{E},
$$

where

$$
\begin{aligned}
Q_{\underline{l}}:= & \prod_{i \in\{1, \ldots, m\}: l_{i}>0} \prod_{\nu=0}^{l_{i}-1}\left(\widehat{\mathcal{D}}_{i}-\nu z\right) \prod_{j \in\{1, \ldots, c\}: l_{m+j}>0} \prod_{\nu=1}^{l_{m+l}}\left(\widehat{\mathcal{L}}_{j}+\nu z\right) \\
& -\underline{q}^{\underline{\underline{l}} .} \prod_{i \in\{1, \ldots, m\}: l_{i}<0} \prod_{\nu=0}^{-l_{i}-1}\left(\widehat{\mathcal{D}}_{i}-\nu z\right) \prod_{j \in\{1, \ldots, c\}: l_{m+j}<0} \prod_{\nu=1}^{-l_{m+l}}\left(\widehat{\mathcal{L}}_{j}+\nu z\right), \\
\widehat{E}:=z^{2} \partial_{z}-\widehat{K}_{\mathbb{V}\left(\mathcal{E}^{\vee}\right)} &
\end{aligned}
$$




\section{Hypergeometric Hodge modules}

Here we write $\mathcal{D}_{i} \in \operatorname{Pic}\left(X_{\Sigma}\right)$ for a line bundle associated with the torus invariant divisor $D_{i}$, where $i=1, \ldots, m$. Let $K \subset R_{\mathbb{C}_{z} \times \mathcal{K} \mathcal{M}^{\circ}}$ be the ideal

$$
K:=\left\{P \in R_{\mathbb{C}_{z} \times \mathcal{K} \mathcal{M}^{\circ}} \mid \exists p \in \mathbb{Z}, k \in \mathbb{N}: \prod_{i=0}^{k} \prod_{j=1}^{c}(\widehat{\mathcal{L}}+p+i) P \in J\right\}
$$

and $\mathcal{K}$ the associated sheaf of ideals in $\mathcal{R}_{\mathbb{C}_{z} \times \mathcal{K} \mathcal{M}^{\circ}}$.

Suppose as above that the bundle $-K_{X_{\Sigma}}-\sum_{j=1}^{l} \mathcal{L}_{j}$ is nef, and moreover that each individual bundle $\mathcal{L}_{j}$ is ample. Then there is a map Mir: $B_{\varepsilon}^{*} \rightarrow H^{0}\left(X_{\Sigma}, \mathbb{C}\right) \times B_{\varepsilon}^{*}$ such that we have an isomorphism of $\mathcal{R}_{\mathbb{C}_{z} \times B_{\varepsilon}^{*} \text {-modules }}$

$$
\left(\mathcal{R}_{\mathbb{C}_{z} \times \mathcal{K} \mathcal{M}^{\circ} / \mathcal{K}}\right)_{\mid \mathbb{C}_{z} \times B_{\varepsilon}^{*}} \stackrel{\cong}{\longrightarrow}\left(\operatorname{id}_{\mathbb{C}_{z}} \times \operatorname{Mir}\right)^{*} \overline{\operatorname{QDM}}\left(X_{\Sigma}, \mathcal{E}\right)
$$

In order to relate the quantum $\mathcal{D}$-module $\overline{\mathrm{QDM}}\left(X_{\Sigma}, \mathcal{E}\right)$ with our results on GKZ systems, we will use the restriction map $\bar{\rho}: \mathcal{K M}^{\circ} \hookrightarrow \Lambda$ as constructed in [RS17, discussion before Definition 6.3]. Then it follows from the results of [RS17, Proposition 6.10], that we have an isomorphism of $\mathcal{R}_{\mathbb{C}_{z} \times \mathcal{K} \mathcal{M}^{\circ-} \text { modules }}$

$$
\mathcal{R}_{\mathbb{C}_{z} \times \mathcal{K} \mathcal{M}^{\circ}} / \mathcal{K} \cong\left(\operatorname{id}_{\mathbb{C}_{z}} \times \bar{\rho}\right)^{*}\left(\widetilde{\phi}\left(G_{0}{ }^{\circ} \widehat{\mathcal{N}}_{A}^{(0, \underline{0}, \underline{0})}\right)\right) .
$$

Now we can deduce from Corollary 6.4 the main result of this section.

ThEOREM 6.6. Consider the above situation of a $k$-dimensional toric variety $X_{\Sigma}$ and globally generated line bundles $\mathcal{L}_{1}, \ldots, \mathcal{L}_{l}$ such that $-K_{X_{\Sigma}}-\mathcal{E}$ is nef, where $\mathcal{E}=\oplus_{j=1}^{l} \mathcal{L}_{j}$ with $\mathcal{L}_{j}$ ample for

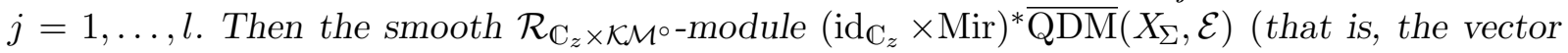
bundle over $\mathbb{C}_{z} \times \mathcal{K M}^{\circ}$ together with its connection operator $\left.\nabla\right)$ underlies a variation of pure polarized non-commutative Hodge structures.

Proof. The strictness of $\widetilde{\phi}$ as shown in Proposition 6.3 shows that $G_{0} \widehat{\mathcal{M}}_{A}^{(-l, \underline{0}, \underline{0})} \cap \operatorname{im}(\widetilde{\phi})=$ $\widetilde{\phi}\left(G_{0} \mathcal{N}^{(0, \underline{0}, \underline{0})}\right)$; hence, by Corollary 6.4 , the module $\widetilde{\phi}\left(G_{0} \mathcal{N}^{(0, \underline{0}, \underline{0})}\right)$ underlies a variation of pure polarized non-commutative Hodge structures on $\Lambda^{0}$. Hence, the assertion follows from the mirror statement of Theorem 6.5.

\section{ACKNowledGements}

We thank Takuro Mochizuki, Claude Sabbah and Uli Walther for many stimulating discussions on the content of this paper.

\section{REFERENCES}

Ado94 A. Adolphson, Hypergeometric functions and rings generated by monomials, Duke Math. J. 73 (1994), no. 2, 269-290; doi:10.1215/S0012-7094-94-07313-4.

Bat93 V.V. Batyrev, Variations of the mixed Hodge structure of affine hypersurfaces in algebraic tori, Duke Math. J. 69 (1993), no. 2, 349-409; doi:10.1215/S0012-7094-93-06917-7.

BGM15 C. Berkesch Zamaere, S. Griffeth and E. Miller, Systems of parameters and holonomicity of Ahypergeometric systems, Pacific J. Math. 276 (2015), no. 2, 281-286; doi:10.2140/pjm. 2015. 276.281.

BH98 W. Bruns and J. Herzog, Cohen-Macaulay rings, Cambridge Stud. Adv. Math., vol. 39 (Cambridge Univ. Press, Cambridge, 1998); doi:10.1017/CB09780511608681. 


\section{T. Reichelt And C. Sevenheck}

Bry86 J.-L. Brylinski, Transformations canoniques, dualité projective, théorie de Lefschetz, transformations de Fourier et sommes trigonométriques, Astérisque 140-141 (1986), 3-134.

CRS19 A. Castaño Domínguez, T. Reichelt and C. Sevenheck, Examples of hypergeometric twistor $\mathcal{D}$ modules, Algebra Number Theory 13 (2019), no. 6, 1415-1442; doi:10.2140/ant.2019.13. 1415.

CS19 A. Castaño Domínguez and C. Sevenheck, Irregular Hodge filtration of some confluent hypergeometric systems, J. Inst. Math. Jussieu, published online on 24 May 2019, doi:10.1017/ S1474748019000288, to appear in print.

DE03 A. D'Agnolo and M. Eastwood, Radon and Fourier transforms for D-modules, Adv. Math. 180 (2003), no. 2, 452-485; doi:10.1016/S0001-8708(03)00011-2.

DS13 M. Dettweiler and C. Sabbah, Hodge theory of the middle convolution, Publ. Res. Inst. Math. Sci. 49 (2013), no. 4, 761-800; doi:10.4171/PRIMS/119.

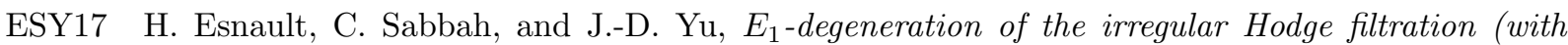
an appendix by Morihiko Saito), J. reine angew. Math. 729 (2017), 171-227; doi:10.1515/ crelle-2014-0118.

Ful93 W. Fulton, Introduction to toric varieties, Ann. of Math. Stud., vol. 131 (Princeton Univ. Press, Princeton, NJ, 1993); doi:10.1515/9781400882526.

GD61 A. Grothendieck and A. Dieudonné, Éléments de géométrie algébrique. III. Étude cohomologique des faisceaux cohérents. Première partie, Publ. Math. Inst. Hautes Études Sci. 11 (1961), 5-167; http://www . numdam.org/item?id=PMIHES_1961__11__5_0.

GGZ87 I. M. Gel'fand, M. I. Graev and A. V. Zelevinskiǔ, Holonomic systems of equations and series of hypergeometric type, Dokl. Akad. Nauk SSSR 295 (1987), no. 1, 14-19.

GKZ90 I. M. Gel'fand, M. M. Kapranov and A. V. Zelevinsky, Generalized Euler integrals and A-hypergeometric functions, Adv. Math. 84 (1990), no. 2, 255-271; doi:10.1016/0001-8708(90) 90048-R.

GZK89 I. M. Gel'fand, A. V. Zelevinskiu and M. M. Kapranov, Hypergeometric functions and toral manifolds, Funct. Anal. Appl. 23 (1989), no. 2, 94-106; doi:10.1007/BF01078777.

Har77 R. Hartshorne, Algebraic geometry, Grad. Texts in Math., vol. 52 (Springer-Verlag, New York Heidelberg, 1977); doi:10.1007/978-1-4757-3849-0.

Hoc72 M. Hochster, Rings of invariants of tori, Cohen-Macaulay rings generated by monomials, and polytopes, Ann. of Math. 96 (1972), 318-337; doi:10.2307/1970791.

HTT08 R. Hotta, K. Takeuchi and T. Tanisaki, D-modules, perverse sheaves, and representation theory, Progr. Math., vol. 236 (Birkhäuser Boston, Inc., Boston, MA, 2008); doi:10.1007/ 978-0-8176-4523-6.

Kat90 N. M. Katz, Exponential sums and differential equations, Ann. of Math. Stud., vol. 124 (Princeton Univ. Press, Princeton, NJ, 1990); doi:10.1515/9781400882434.

KKP17 L. Katzarkov, M. Kontsevich and T. Pantev, Bogomolov-Tian-Todorov theorems for LandauGinzburg models, J. Differential Geom. 105 (2017), no. 1, 55-117; doi:10.4310/jdg/ 1483655860.

MM04 P. Maisonobe and Z. Mebkhout, Le théorème de comparaison pour les cycles évanescents, Éléments de la théorie des systèmes différentiels géométriques, Sémin. Congr., vol. 8 (Soc. Math. France, Paris, 2004), 311-389; http://emis.ams.org/journals/SC/2004/8/html/smf _ sem-cong_8_311-389.html.

MM17 E. Mann and T. Mignon, Quantum D-modules for toric nef complete intersections, Internat. J. Math. 28 (2017), no. 6, 1750047, 49 pp.; doi:10.1142/S0129167X17500471.

MMW05 L.F. Matusevich, E. Miller and U. Walther, Homological methods for hypergeometric families, J. Amer. Math. Soc. 18 (2005), no. 4, 919-941; doi:10.1090/S0894-0347-05-00488-1.

Moc15a T. Mochizuki, Mixed twistor D-modules, Lecture Notes in Math., vol. 2125 (Springer, Cham, 2015); doi:10.1007/978-3-319-10088-3. 


\section{Hypergeometric Hodge modules}

Moc15b _ Twistor property of GKZ-hypergeometric systems, 2015, arXiv:1501.04146.

MS05 E. Miller and B. Sturmfels, Combinatorial commutative algebra, Grad. Texts in Math., vol. 227 (Springer-Verlag, New York, 2005); doi:10.1007/b138602.

Rei14 T. Reichelt, Laurent polynomials, GKZ-hypergeometric systems and mixed Hodge modules, Compos. Math. 150 (2014), no. 6, 911-941; doi:10.1112/S0010437X13007744.

RS15 T. Reichelt and C. Sevenheck, Logarithmic Frobenius manifolds, hypergeometric systems and quantum D-modules, J. Algebraic Geom. 24 (2015), no. 2, 201-281; doi:10.1090/ S1056-3911-2014-00625-1.

RS17 _ Non-affine Landau-Ginzburg models and intersection cohomology, Ann. Sci. Éc. Norm. Supér. (4) 50 (2017), no. 3, 665-753; doi:10.24033/asens. 2330.

RSW18 T. Reichelt, C. Sevenheck and U. Walther, On the b-functions of hypergeometric systems, Int. Math. Res. Not. 2018 (2018), no. 21, 6535-6555; doi:10.1093/imrn/rnx039.

Sab05 C. Sabbah, Polarizable twistor D-modules, Astérisque 300 (2005).

Sab08_, Fourier-Laplace transform of a variation of polarized complex Hodge structure, J. reine angew. Math. 621 (2008), 123-158; doi:10.1515/CRELLE. 2008.060.

Sab11 , Non-commutative Hodge structures, Ann. Inst. Fourier (Grenoble) 61 (2011), no. 7, 2681-2717; doi:10.5802/aif.2790.

Sab18_, Irregular Hodge theory (with the collaboration of Jeng-Daw Yu), Mém. Soc. Math. Fr. (N.S.) (2018), no. 156; https://smf .emath.fr/sites/default/files/2018-11/smf_mem-ns_ 156__sample.pdf.

Sai88 M. Saito, Modules de Hodge polarisables, Publ. Res. Inst. Math. Sci. 24 (1988), no. 6, 849-995; doi:10.2977/prims/1195173930.

Sai90 _ Mixed Hodge modules, Publ. Res. Inst. Math. Sci. 26 (1990), no. 2, 221-333; doi: 10.2977/prims/1195171082.

Sai93_, On b-function, spectrum and rational singularity, Math. Ann. 295 (1993), no. 1, 51-74; doi:10.1007/BF01444876.

Sai94_, On the theory of mixed Hodge modules, Selected Papers on Number Theory, Algebraic Geometry, and Differential Geometry, Amer. Math. Soc. Transl. Ser. 2, vol. 160 (Amer. Math. Soc., Providence, RI, 1994), 47-61; doi:10.1090/trans2/160/04.

SST00 M. Saito, B. Sturmfels and N. Takayama, Gröbner deformations of hypergeometric differential equations, Algorithms and Computation in Mathematics, vol. 6 (Springer-Verlag, Berlin, 2000) doi:10.1007/978-3-662-04112-3.

Sti98 J. Stienstra, Resonant hypergeometric systems and mirror symmetry, Integrable Systems and Algebraic Geometry (Kobe/Kyoto, 1997) (World Sci. Publ., River Edge, NJ, 1998), 412-452.

SW08 M. Schulze and U. Walther, Irregularity of hypergeometric systems via slopes along coordinate subspaces, Duke Math. J. 142 (2008), no. 3, 465-509; doi:10.1215/00127094-2008-011.

SW09 - Hypergeometric D-modules and twisted Gauß-Manin systems, J. Algebra 322 (2009), no. 9, 3392-3409; doi:10.1016/j.jalgebra.2008.09.010.

Wal07 U. Walther, Duality and monodromy reducibility of A-hypergeometric systems, Math. Ann. 338 (2007), no. 1, 55-74; doi:10.1007/s00208-006-0067-x.

Thomas Reichelt treichelt@mathi.uni-heidelberg.de

Mathematisches Institut, Universität Heidelberg, 69120 Heidelberg, Germany

Christian Sevenheck christian.sevenheck@mathematik.tu-chemnitz.de

Fakultät für Mathematik, Technische Universität Chemnitz, 09107 Chemnitz, Germany 